\title{
Litosetoenins A-E, Diterpenoids from the Soft Coral Litophyton setoensis, Backbone-Rearranged through Divergent Cyclization achieved by Epoxide Reactivity Inversion
}

\author{
Song-Wei Li, ${ }^{\dagger, 1}$ I. Wayan Mudianta, $\$, 1$ Cristina Cuadrado, ${ }^{\ddagger}$ Geng Li, ${ }^{\dagger}$ Gede A. \\ Yudasmara, ${ }^{\S}$ Gede I. Setiabudi, ${ }^{\S}$ Antonio H. Daranas, ${ }^{\uparrow, *}$ Yue-Wei Guo ${ }^{\dagger, *}$ \\ $\dagger$ State Key Laboratory of Drug Research, Shanghai Institute of Materia Medica, Chinese Academy of Sciences, 555 \\ Zu Chong Zhi Road, Zhangjiang Hi-Tech Park, Shanghai 201203, China \\ * Department of Chemistry, Universitas Pendidikan Ganesha, Bali 81116, Indonesia \\ 『 Instituto de Productos Naturales y Agrobiología, Consejo Superior de Investigaciones Científicas (IPNA-CSIC), La Laguna, \\ 38206 Tenerife, Spain \\ $\S$ Study Program of Aquaculture, Universitas Pendidikan Ganesha, Bali 81116, Indonesia \\ * Corresponding authors: ywguo@simm.ac.cn (Y.-W. Guo); adaranas@ipna.csic.es (A. H. Daranas)
}


Table of Contents

Figure S1. ${ }^{1} \mathrm{H}$ NMR spectrum of $1\left(500 \mathrm{MHz}, \mathrm{CDCl}_{3}\right) \quad$ S6

Figure S2. ${ }^{13} \mathrm{C}\left\{{ }^{1} \mathrm{H}\right\}$ NMR spectrum of $\mathbf{1}\left(125 \mathrm{MHz}, \mathrm{CDCl}_{3}\right) \quad$ S6

Figure S3. HSQC spectrum of $\mathbf{1}\left(500 \mathrm{MHz}, \mathrm{CDCl}_{3}\right) \quad$ S7

Figure S4. $\mathrm{HMBC}$ spectrum of $1\left(500 \mathrm{MHz}, \mathrm{CDCl}_{3}\right) \quad$ S7

Figure S5. ${ }^{1} \mathrm{H}-{ }^{1} \mathrm{H}$ COSY spectrum of $1\left(500 \mathrm{MHz}, \mathrm{CDCl}_{3}\right) \quad$ S8

Figure S6. NOESY spectrum of $1\left(500 \mathrm{MHz}, \mathrm{CDCl}_{3}\right) \quad$ S8

Figure S7. HR-ESI-MS spectrum of $1 \quad$ S9

Figure S8. IR spectrum of $1 \quad$ S9

Figure S9. ${ }^{1} \mathrm{H}$ NMR spectrum of $2\left(500 \mathrm{MHz}, \mathrm{CDCl}_{3}\right) \quad$ S10

Figure S10. ${ }^{13} \mathrm{C}\left\{{ }^{1} \mathrm{H}\right\}$ NMR spectrum of $2\left(125 \mathrm{MHz}, \mathrm{CDCl}_{3}\right) \quad \mathrm{S} 10$

Figure S11. HSQC spectrum of $2\left(500 \mathrm{MHz}, \mathrm{CDCl}_{3}\right) \quad \mathrm{S} 11$

Figure S12. HMBC spectrum of $2\left(500 \mathrm{MHz}, \mathrm{CDCl}_{3}\right) \quad \mathrm{S} 11$

Figure S13. ${ }^{1} \mathrm{H}-{ }^{1} \mathrm{H}$ COSY spectrum of $2\left(500 \mathrm{MHz}, \mathrm{CDCl}_{3}\right) \quad \mathrm{S} 12$

Figure S14. NOESY spectrum of $2\left(500 \mathrm{MHz}, \mathrm{CDCl}_{3}\right) \quad \mathrm{S} 12$

$\begin{array}{ll}\text { Figure S15. HR-ESI-MS spectrum of } 2 & \text { S13 }\end{array}$

Figure S16. IR spectrum of $2 \quad$ S13

Figure S17. ${ }^{1} \mathrm{H}$ NMR spectrum of $3\left(500 \mathrm{MHz}, \mathrm{CDCl}_{3}\right) \quad \mathrm{S} 14$

Figure S18. ${ }^{13} \mathrm{C}\left\{{ }^{1} \mathrm{H}\right\}$ NMR spectrum of $\mathbf{3}\left(125 \mathrm{MHz}, \mathrm{CDCl}_{3}\right) \quad \mathrm{S} 14$

Figure S19. HSQC spectrum of $3\left(500 \mathrm{MHz}, \mathrm{CDCl}_{3}\right) \quad \mathrm{S} 15$

$\begin{array}{ll}\text { Figure S20. } \mathrm{HMBC} \text { spectrum of } \mathbf{3}\left(500 \mathrm{MHz}, \mathrm{CDCl}_{3}\right) & \mathrm{S} 15\end{array}$

Figure S21. ${ }^{1} \mathrm{H}-{ }^{1} \mathrm{H}$ COSY spectrum of $3\left(500 \mathrm{MHz}, \mathrm{CDCl}_{3}\right) \quad \mathrm{S} 16$

Figure S22. NOESY spectrum of $\mathbf{3}\left(500 \mathrm{MHz}, \mathrm{CDCl}_{3}\right) \quad \mathrm{S} 16$

$\begin{array}{ll}\text { Figure S23. HR-ESI-MS spectrum of } 3 & \text { S17 }\end{array}$

$\begin{array}{ll}\text { Figure S24. IR spectrum of } 3 & \text { S17 }\end{array}$

Figure S25. ${ }^{1} \mathrm{H}$ NMR spectrum of $4\left(500 \mathrm{MHz}, \mathrm{CDCl}_{3}\right) \quad \mathrm{S} 18$

Figure S26. ${ }^{13} \mathrm{C}\left\{{ }^{1} \mathrm{H}\right\}$ NMR spectrum of $4\left(125 \mathrm{MHz}, \mathrm{CDCl}_{3}\right) \quad \mathrm{S} 18$

Figure S27. HSQC spectrum of $4\left(500 \mathrm{MHz}, \mathrm{CDCl}_{3}\right) \quad \mathrm{S} 19$

Figure S28. HMBC spectrum of $4\left(500 \mathrm{MHz}, \mathrm{CDCl}_{3}\right) \quad \mathrm{S} 19$

Figure S29. ${ }^{1} \mathrm{H}-{ }^{1} \mathrm{H}$ COSY spectrum of $4\left(500 \mathrm{MHz}, \mathrm{CDCl}_{3}\right) \quad$ S20

Figure S30. NOESY spectrum of $4\left(500 \mathrm{MHz}, \mathrm{CDCl}_{3}\right) \quad$ S20

$\begin{array}{ll}\text { Figure S31. HR-ESI-MS spectrum of } 4 & \text { S21 }\end{array}$

$\begin{array}{ll}\text { Figure S32. IR spectrum of } 4 & \text { S21 }\end{array}$

Figure S33. ${ }^{1} \mathrm{H}$ NMR spectrum of $5\left(500 \mathrm{MHz}, \mathrm{CDCl}_{3}\right) \quad$ S22

Figure S34. ${ }^{13} \mathrm{C}\left\{{ }^{1} \mathrm{H}\right\}$ NMR spectrum of $5\left(125 \mathrm{MHz}, \mathrm{CDCl}_{3}\right) \quad$ S22

$\begin{array}{ll}\text { Figure S35. HSQC spectrum of } \mathbf{5}\left(500 \mathrm{MHz}, \mathrm{CDCl}_{3}\right) & \mathrm{S} 23\end{array}$

Figure S36. HMBC spectrum of $5\left(500 \mathrm{MHz}, \mathrm{CDCl}_{3}\right) \quad$ S23

Figure S37. ${ }^{1} \mathrm{H}-{ }^{1} \mathrm{H}$ COSY spectrum of $5\left(500 \mathrm{MHz}, \mathrm{CDCl}_{3}\right) \quad \mathrm{S} 24$

Figure S38. NOESY spectrum of $5\left(500 \mathrm{MHz}, \mathrm{CDCl}_{3}\right) \quad$ S24

$\begin{array}{ll}\text { Figure S39. HR-ESI-MS spectrum of } 5 & \text { S25 }\end{array}$

$\begin{array}{ll}\text { Figure S40. IR spectrum of } 5 & \text { S25 }\end{array}$

Figure S41. ${ }^{1} \mathrm{H}$ NMR spectrum of $\mathbf{5 a}\left(500 \mathrm{MHz}, \mathrm{CDCl}_{3}\right) \quad$ S26

Figure $\mathbf{S 4 2 .}{ }^{13} \mathrm{C}\left\{{ }^{1} \mathrm{H}\right\}$ NMR spectrum of $\mathbf{5 a}\left(125 \mathrm{MHz}, \mathrm{CDCl}_{3}\right) \quad$ S26

Figure S43. HSQC spectrum of $\mathbf{5 a}\left(500 \mathrm{MHz}, \mathrm{CDCl}_{3}\right) \quad$ S27

$\begin{array}{ll}\text { Figure S44. } \mathrm{HMBC} \text { spectrum of } \mathbf{5 a}\left(500 \mathrm{MHz}, \mathrm{CDCl}_{3}\right) & \mathrm{S} 27\end{array}$

Figure S45. ${ }^{1} \mathrm{H}-{ }^{1} \mathrm{H}$ COSY spectrum of $\mathbf{5 a}\left(500 \mathrm{MHz}, \mathrm{CDCl}_{3}\right) \quad$ S28

Figure S46. NOESY spectrum of $5 \mathbf{a}\left(500 \mathrm{MHz}, \mathrm{CDCl}_{3}\right) \quad$ S28

$\begin{array}{ll}\text { Figure S47. HR-ESI-MS spectrum of 5a } & \text { S29 }\end{array}$

$\begin{array}{ll}\text { Figure S48. IR spectrum of 5a } & \text { S29 }\end{array}$

Figure S49. ${ }^{1} \mathrm{H}$ NMR spectrum of $\mathbf{5 b}\left(500 \mathrm{MHz}, \mathrm{CDCl}_{3}\right) \quad$ S30 
Figure S50. ${ }^{13} \mathrm{C}\left\{{ }^{1} \mathrm{H}\right\}$ NMR spectrum of $\mathbf{5 b}\left(125 \mathrm{MHz}, \mathrm{CDCl}_{3}\right) \quad \mathrm{S} 30$

Figure S51. HSQC spectrum of $\mathbf{5 b}\left(500 \mathrm{MHz}, \mathrm{CDCl}_{3}\right) \quad$ S31

Figure S52. HMBC spectrum of $\mathbf{5 b}\left(500 \mathrm{MHz}, \mathrm{CDCl}_{3}\right) \quad$ S31

Figure S53. ${ }^{1} \mathrm{H}-{ }^{1} \mathrm{H}$ COSY spectrum of $\mathbf{5 b}\left(500 \mathrm{MHz}, \mathrm{CDCl}_{3}\right) \quad \mathrm{S} 32$

Figure S54. NOESY spectrum of $\mathbf{5 b}\left(500 \mathrm{MHz}, \mathrm{CDCl}_{3}\right) \quad \mathrm{S} 32$

$\begin{array}{ll}\text { Figure S55. HR-ESI-MS spectrum of } \mathbf{5 b} & \text { S33 }\end{array}$

Figure S56. IR spectrum of $\mathbf{5 b} \quad$ S33

Figure S57. ${ }^{1} \mathrm{H}$ NMR spectrum of $6\left(500 \mathrm{MHz}, \mathrm{CDCl}_{3}\right) \quad \mathrm{S} 34$

Figure S58. ${ }^{13} \mathrm{C}\left\{{ }^{1} \mathrm{H}\right\}$ NMR spectrum of $6\left(125 \mathrm{MHz}, \mathrm{CDCl}_{3}\right) \quad \mathrm{S} 34$

Figure S59. ${ }^{1} \mathrm{H}$ NMR spectrum of $6 \mathbf{6 a}\left(500 \mathrm{MHz}, \mathrm{CDCl}_{3}\right) \quad \mathrm{S} 35$

Figure S60. ${ }^{13} \mathrm{C}\left\{{ }^{1} \mathrm{H}\right\}$ NMR spectrum of $6 \mathbf{6}\left(125 \mathrm{MHz}, \mathrm{CDCl}_{3}\right) \quad \mathrm{S} 35$

Figure S61. ${ }^{1} \mathrm{H}$ NMR spectrum of $7\left(400 \mathrm{MHz}, \mathrm{CDCl}_{3}\right) \quad$ S36

Figure S62. ${ }^{1} \mathrm{H}$ NMR spectrum of $8\left(500 \mathrm{MHz}, \mathrm{CDCl}_{3}\right) \quad$ S36

Figure S63. ${ }^{13} \mathrm{C}\left\{{ }^{1} \mathrm{H}\right\}$ NMR spectrum of $8\left(125 \mathrm{MHz}, \mathrm{CDCl}_{3}\right) \quad$ S37

Figure S64. ${ }^{1} \mathrm{H}$ NMR spectrum of $9\left(500 \mathrm{MHz}, \mathrm{CDCl}_{3}\right) \quad$ S37

Figure S65. ${ }^{13} \mathrm{C}\left\{{ }^{1} \mathrm{H}\right\}$ NMR spectrum of $9\left(125 \mathrm{MHz}, \mathrm{CDCl}_{3}\right) \quad \mathrm{S} 38$

Figure S66. ${ }^{1} \mathrm{H}$ NMR spectrum of $10\left(500 \mathrm{MHz}, \mathrm{CDCl}_{3}\right) \quad$ S38

Figure S67. ${ }^{13} \mathrm{C}\left\{{ }^{1} \mathrm{H}\right\} \mathrm{NMR}$ spectrum of $\mathbf{1 0}\left(125 \mathrm{MHz}, \mathrm{CDCl}_{3}\right) \quad \mathrm{S} 39$

Figure S68. ${ }^{1} \mathrm{H}$ NMR spectrum of $11\left(400 \mathrm{MHz}, \mathrm{CDCl}_{3}\right) \quad \mathrm{S} 39$

Table S1. ${ }^{1} \mathrm{H}$ and ${ }^{13} \mathrm{C}$ NMR data of compounds 1, 2, 3, 4 and $5 \quad$ S41

$\begin{array}{ll}\text { Figure S69. Structures of isomers 1-8 of compound } 1 & \text { S43 }\end{array}$

$\begin{array}{ll}\text { Figure S70. Structures of isomers 1-8 of compound } 2 & \text { S44 }\end{array}$

Figure S71. Structures of isomers 1-16 of compound $3 \quad$ S45

Figure S72. Structures of isomers 1-32 of compound $4 \quad$ S46

Figure S73. Structures of isomers 1-8 of compound $5 \quad$ S48

Figure S74. Structures of isomers 1-4 of compound isoelisabethatriene $\quad$ S49

Table S2. Boltzmann averaged GIAO isotropic magnetic shielding constants $(\sigma)$ of isomers 1-8 calculated at the PCM/mPW1PW91/6-31+G**//B3LYP/6-31G* level of theory for compound 1

S50

Table S3. Boltzmann averaged GIAO isotropic magnetic shielding constants $(\sigma)$ of isomers 1-8 calculated at the PCM/mPW1PW91/6-31+G**//B3LYP/6-31G* level of theory for compound 2

S51

Table S4. Boltzmann averaged GIAO isotropic magnetic shielding constants $(\sigma)$ of isomers 1-16 calculated at the PCM/mPW1PW91/6-31+G**//B3LYP/6-31G* level of theory for compound $\mathbf{3}$

S52

Table S5. Boltzmann averaged GIAO isotropic magnetic shielding constants $(\sigma)$ of isomers 1-32 calculated at the PCM/mPW1PW91/6-31+G**//B3LYP/6-31G* level of theory for compound 4

S54

Table S6. Boltzmann averaged GIAO isotropic magnetic shielding constants $(\sigma)$ of isomers 1-8 calculated at the PCM/mPW1PW91/6-31+G**//B3LYP/6-31G* level of theory for compound $\mathbf{5}$

S58

Table S7. Boltzmann averaged GIAO isotropic magnetic shielding constants $(\sigma)$ of isomers 1-4 calculated at the PCM/mPW1PW91/6-31+G**//B3LYP/6-31G* level of theory for compound isoelisabethatriene (vacuum)

S59

Table S8. Boltzmann averaged GIAO isotropic magnetic shielding constants $(\sigma)$ of isomers 1-4 calculated at the PCM/mPW1PW91/6-31+G**//B3LYP/6-31G* level of theory for compound isoelisabethatriene in benzene

S60

Table S9. Unscaled and scaled chemical shifts and its errors computed for isomers 1-8 at the PCM/mPW1PW91/6-31+G**//B3LYP/6-31G* level of theory for compound 1 S61 
Table S10. Unscaled and scaled chemical shifts and its errors computed for isomers 1-8 at the PCM/mPW1PW91/6-31+G**//B3LYP/6-31G* level of theory for compound 2 S64

Table S11. Unscaled and scaled chemical shifts and its errors computed for isomers 1-16 at the PCM/mPW1PW91/6-31+G**//B3LYP/6-31G* level of theory for compound 3 S67

Table S12. Unscaled and scaled chemical shifts and its errors computed for isomers 1-32 at the PCM/mPW1PW91/6-31+G**//B3LYP/6-31G* level of theory for compound 4 S73

Table S13. Unscaled and scaled chemical shifts and its errors computed for isomers 1-8 at the PCM/mPW1PW91/6-31+G**//B3LYP/6-31G* level of theory for compound 5 S86

Table S14. Unscaled and scaled chemical shifts and its errors computed for isomers 1-8 at the PCM/mPW1PW91/6-31+G**//B3LYP/6-31G* level of theory for compound isoelisabethatriene (vacuum)

S89

Table S15. Unscaled and scaled chemical shifts and its errors computed for isomers 1-8 at the PCM/mPW1PW91/6-31+G**//B3LYP/6-31G* level of theory for compound isoelisabethatriene benzene

S92

Table S16. DP4+ results obtained using experimental data of compound 1 versus isomers 1-8

S95

Table S17. DP4+ results obtained using experimental data of compound 2 versus isomers 1-8

Table S18. DP4+ results obtained using experimental data of compound 3 versus isomers 1-16

S96

Table S19. DP4+ results obtained using experimental data of compound 4 versus isomers $1-32$

Table S20. DP4+ results obtained using experimental data of compound 5 versus isomers 1-8

Table S21. DP4+ results obtained using experimental data of compound isoelisabethatriene (vacuum) versus isomers 1-4

Table S22. DP4+ results obtained using experimental data of compound isoelisabethatriene (benzene) versus isomers 1-4

S98

Figure S75. Compound 1 correlation plots between ${ }^{13} \mathrm{C}$ isotropic magnetic shielding values computed at the $\mathrm{PCM} / \mathrm{mPW} 1 \mathrm{PW} 91 / 6-31+\mathrm{G}^{* *}$ level of theory and experimental ${ }^{13} \mathrm{C}$ chemical shifts for isomers $1-8$

S99

Figure S76. Compound 1 correlation plots between ${ }^{1} \mathrm{H}$ isotropic magnetic shielding values computed at the PCM/mPW1PW91/6-31+G** level of theory and experimental ${ }^{1} \mathrm{H}$ chemical shifts for isomers 1-8

S101

Figure S77. Compound 2 correlation plots between ${ }^{13} \mathrm{C}$ isotropic magnetic shielding values computed at the $\mathrm{PCM} / \mathrm{mPW} 1 \mathrm{PW} 91 / 6-31+\mathrm{G}^{* *}$ level of theory and experimental ${ }^{13} \mathrm{C}$ chemical shifts for isomers 1-8

S102

Figure S78. Compound 2 correlation plots between ${ }^{1} \mathrm{H}$ isotropic magnetic shielding values computed at the $\mathrm{PCM} / \mathrm{mPW} 1 \mathrm{PW} 91 / 6-31+\mathrm{G}^{* *}$ level of theory and experimental ${ }^{1} \mathrm{H}$ chemical shifts for isomers $1-8$

S104

Figure S79. Compound 3 correlation plots between ${ }^{13} \mathrm{C}$ isotropic magnetic shielding values computed at the $\mathrm{PCM} / \mathrm{mPW} 1 \mathrm{PW} 91 / 6-31+\mathrm{G}^{* *}$ level of theory and experimental ${ }^{13} \mathrm{C}$ chemical shifts for isomers $1-16$

$\mathrm{S} 105$

Figure S80. Compound 3 correlation plots between ${ }^{1} \mathrm{H}$ isotropic magnetic shielding values computed at the PCM/mPW1PW91/6-31+G** level of theory and experimental ${ }^{1} \mathrm{H}$ chemical shifts for isomers $1-16$

S 107 
Figure S81. Compound 4 correlation plots between ${ }^{13} \mathrm{C}$ isotropic magnetic shielding values computed at the $\mathrm{PCM} / \mathrm{mPW} 1 \mathrm{PW} 91 / 6-31+\mathrm{G}^{* *}$ level of theory and experimental ${ }^{13} \mathrm{C}$ chemical shifts for isomers $1-32$

Figure S82. Compound 4 correlation plots between ${ }^{1} \mathrm{H}$ isotropic magnetic shielding values computed at the PCM/mPW1PW91/6-31+G** level of theory and experimental ${ }^{1} \mathrm{H}$ chemical shifts for isomers 1-32

Figure S83. Compound 5 correlation plots between ${ }^{13} \mathrm{C}$ isotropic magnetic shielding values computed at the $\mathrm{PCM} / \mathrm{mPW} 1 \mathrm{PW} 91 / 6-31+\mathrm{G}^{* *}$ level of theory and experimental ${ }^{13} \mathrm{C}$ chemical shifts for isomers $1-8$

Figure S84. Compound 5 correlation plots between ${ }^{1} \mathrm{H}$ isotropic magnetic shielding values computed at the $\mathrm{PCM} / \mathrm{mPW} 1 \mathrm{PW} 91 / 6-31+\mathrm{G}^{* *}$ level of theory and experimental ${ }^{1} \mathrm{H}$ chemical shifts for isomers 1-8

Figure S85. Compound isoelisabethatriene (vacuum) correlation plots between ${ }^{13} \mathrm{C}$ isotropic magnetic shielding values computed at the PCM/mPW1PW91/6-31+G** level of theory and experimental ${ }^{13} \mathrm{C}$ chemical shifts for isomers 1-4 S119 Figure S86. Compound isoelisabethatriene (vacuum) correlation plots between ${ }^{1} \mathrm{H}$ isotropic magnetic shielding values computed at the PCM/mPW1PW91/6-31+G** level of theory and experimental ${ }^{1} \mathrm{H}$ chemical shifts for isomers 1-4 S120 Figure S87. Compound isoelisabethatriene (benzene) correlation plots between ${ }^{13} \mathrm{C}$ isotropic magnetic shielding values computed at the PCM/mPW1PW91/6-31+G** level of theory and experimental ${ }^{13} \mathrm{C}$ chemical shifts for isomers 1-4 S121 Figure S88. Compound isoelisabethatriene (benzene) correlation plots between ${ }^{1} \mathrm{H}$ isotropic magnetic shielding values computed at the PCM/mPW1PW91/6-31+G** level of theory and experimental ${ }^{1} \mathrm{H}$ chemical shifts for isomers $1-4$ 


\section{Experimental spectra}

Figure S1. ${ }^{1} \mathrm{H}$ NMR spectrum of $1\left(500 \mathrm{MHz}, \mathrm{CDCl}_{3}\right)$

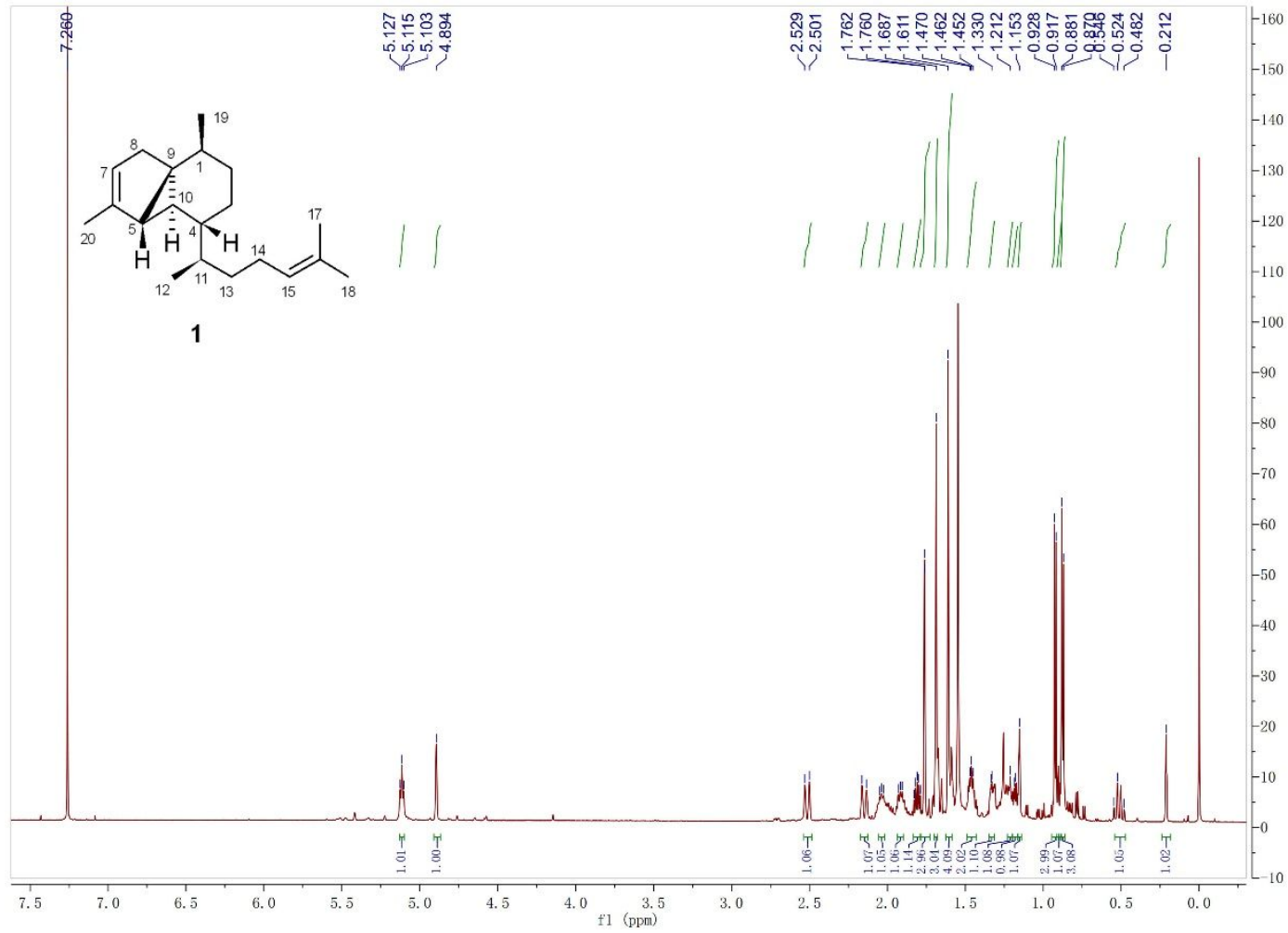

Figure S2. ${ }^{13} \mathrm{C}\left\{{ }^{1} \mathrm{H}\right\}$ NMR spectrum of $\mathbf{1}\left(125 \mathrm{MHz}, \mathrm{CDCl}_{3}\right)$

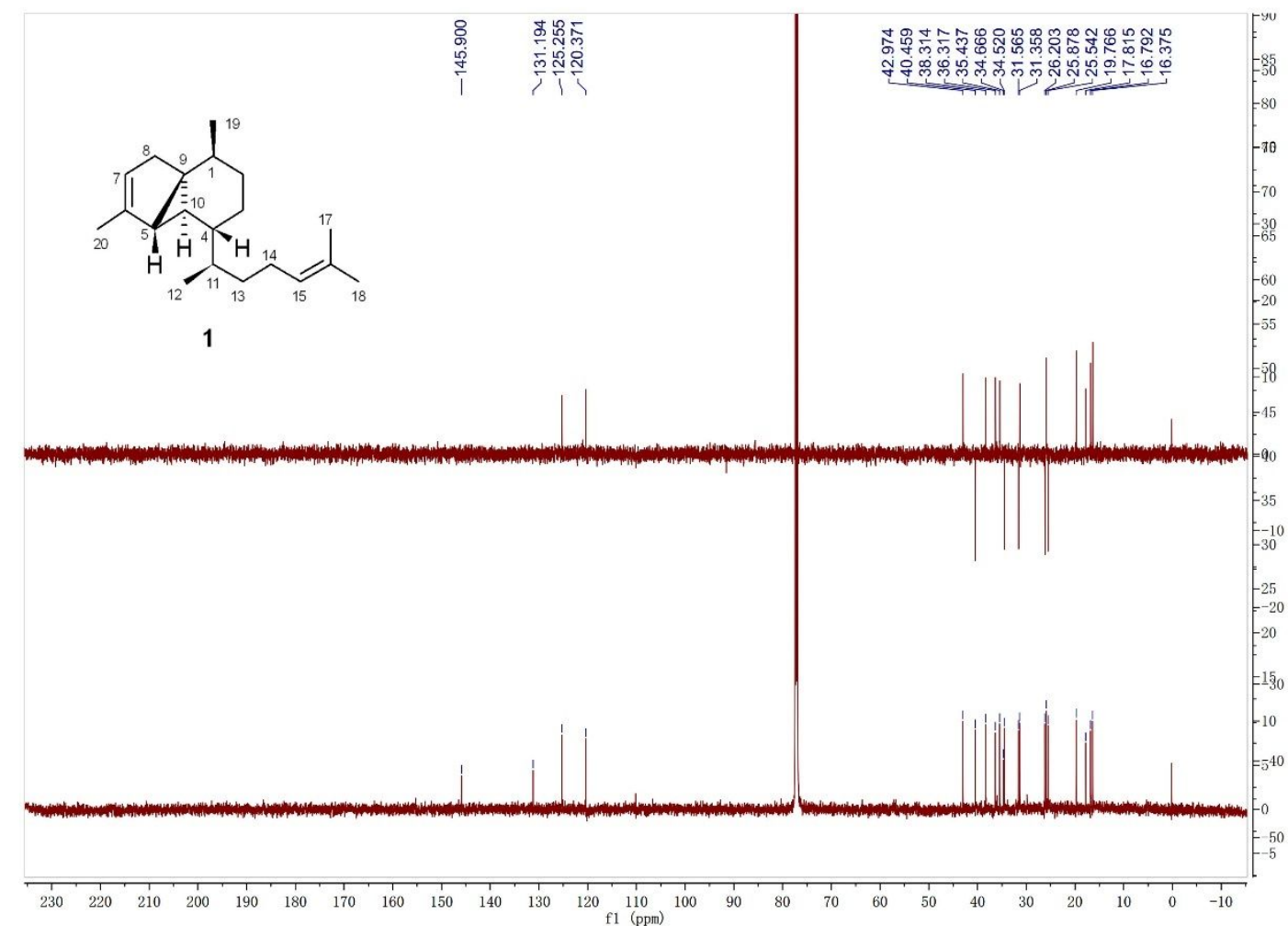


Figure S3. HSQC spectrum of $1\left(500 \mathrm{MHz}, \mathrm{CDCl}_{3}\right)$

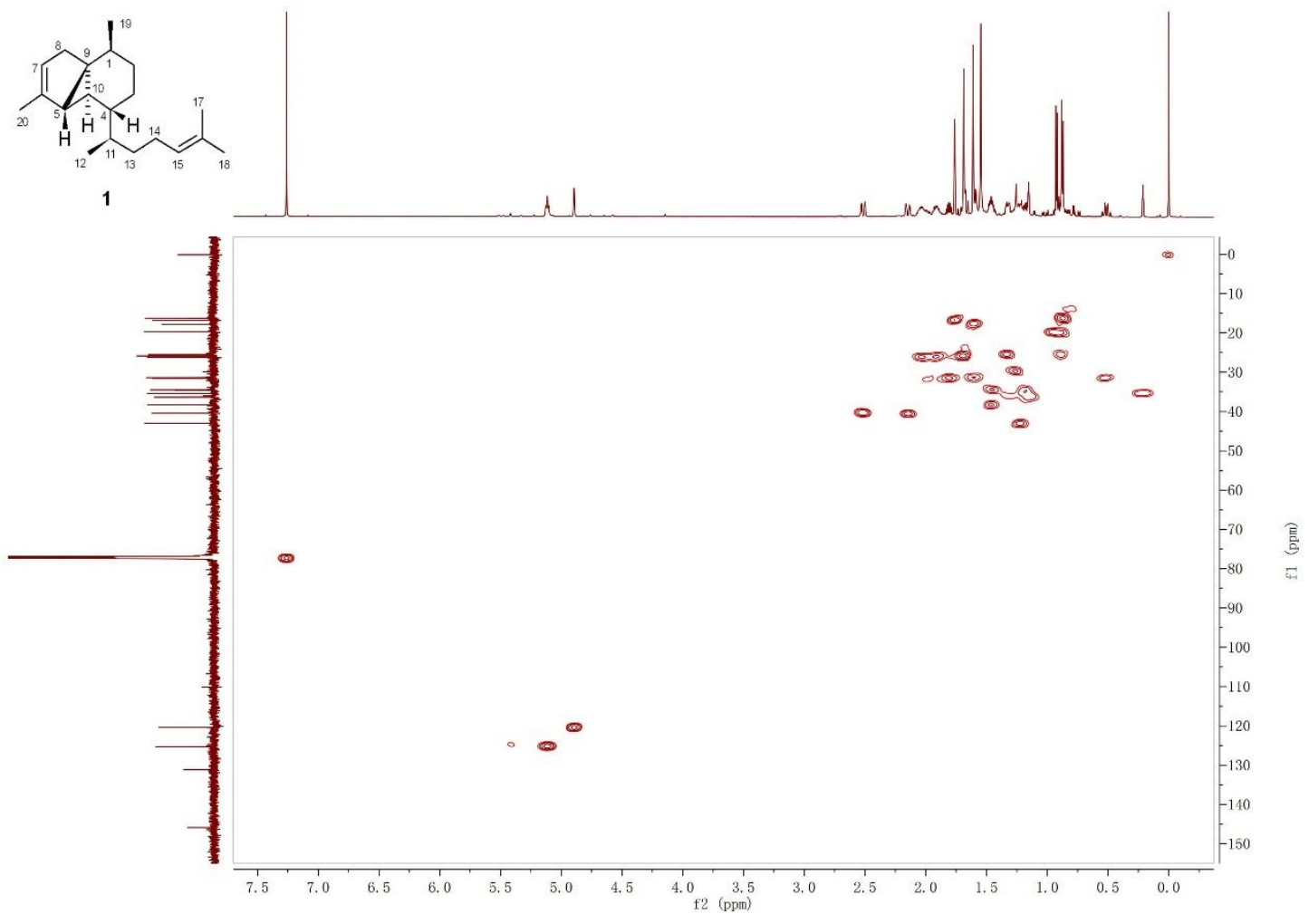

Figure S4. HMBC spectrum of $1\left(500 \mathrm{MHz}, \mathrm{CDCl}_{3}\right)$

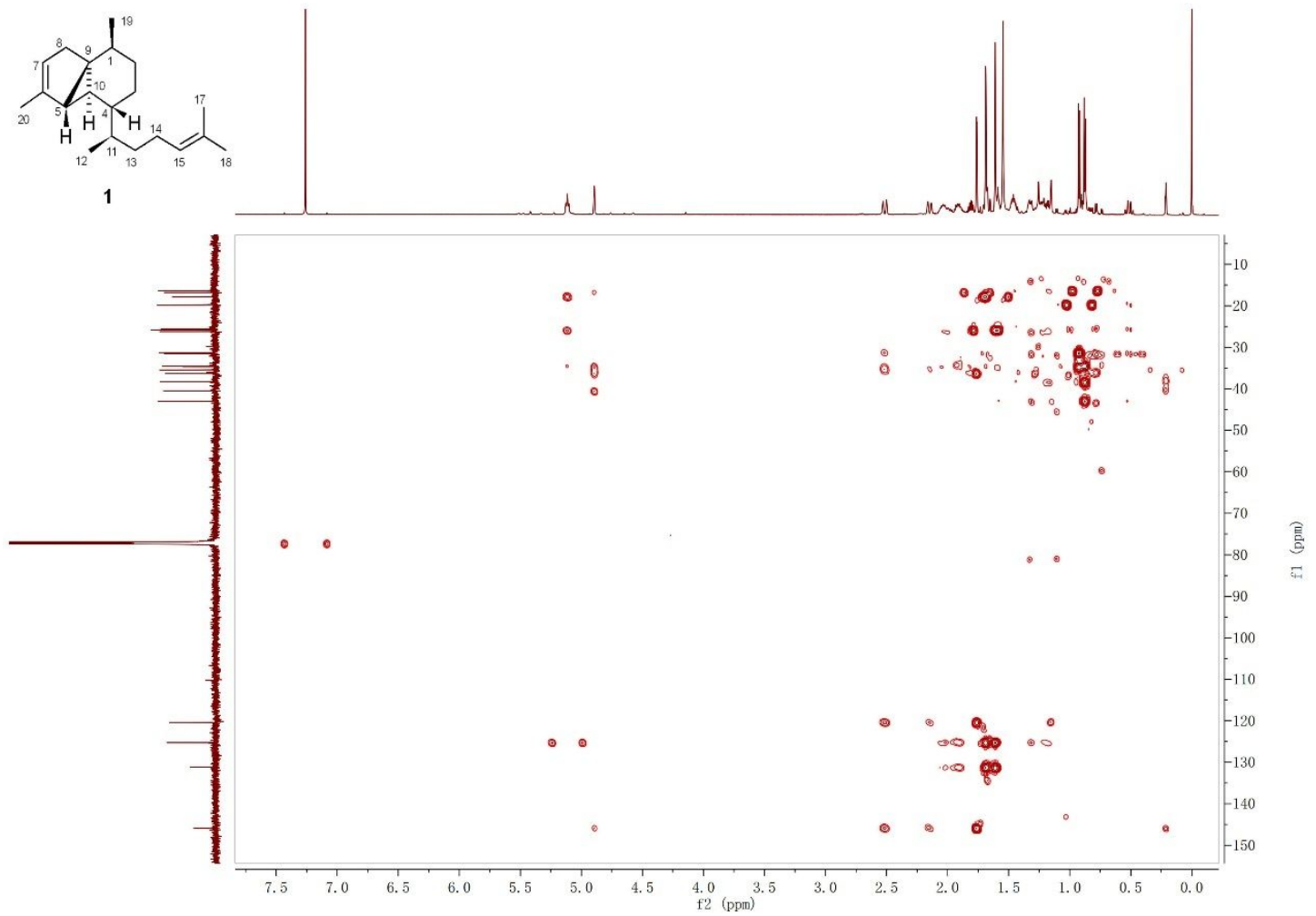


Figure S5. ${ }^{1} \mathrm{H}-{ }^{1} \mathrm{H}$ COSY spectrum of $1\left(500 \mathrm{MHz}, \mathrm{CDCl}_{3}\right)$

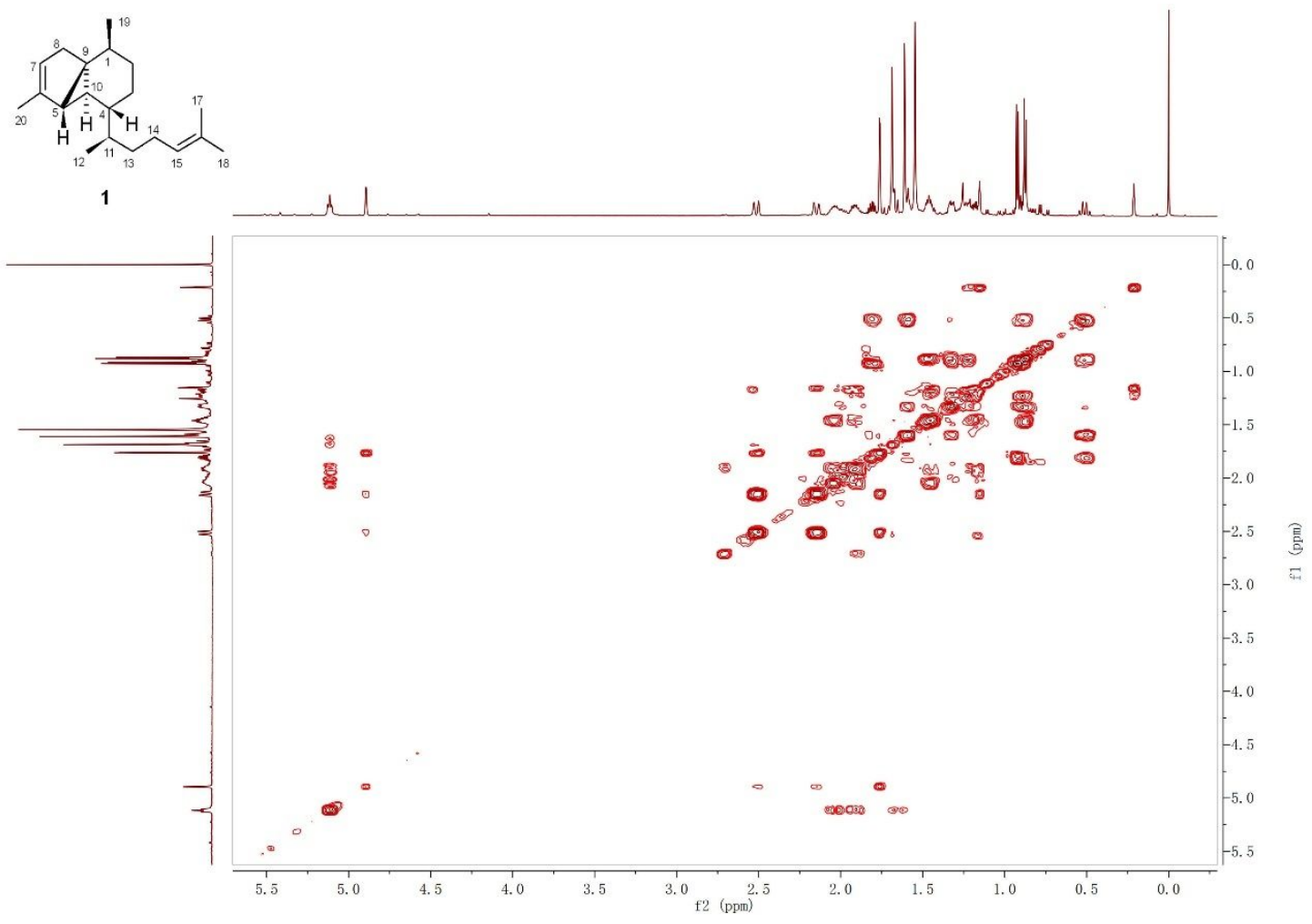

Figure S6. NOESY spectrum of $1\left(500 \mathrm{MHz}, \mathrm{CDCl}_{3}\right)$

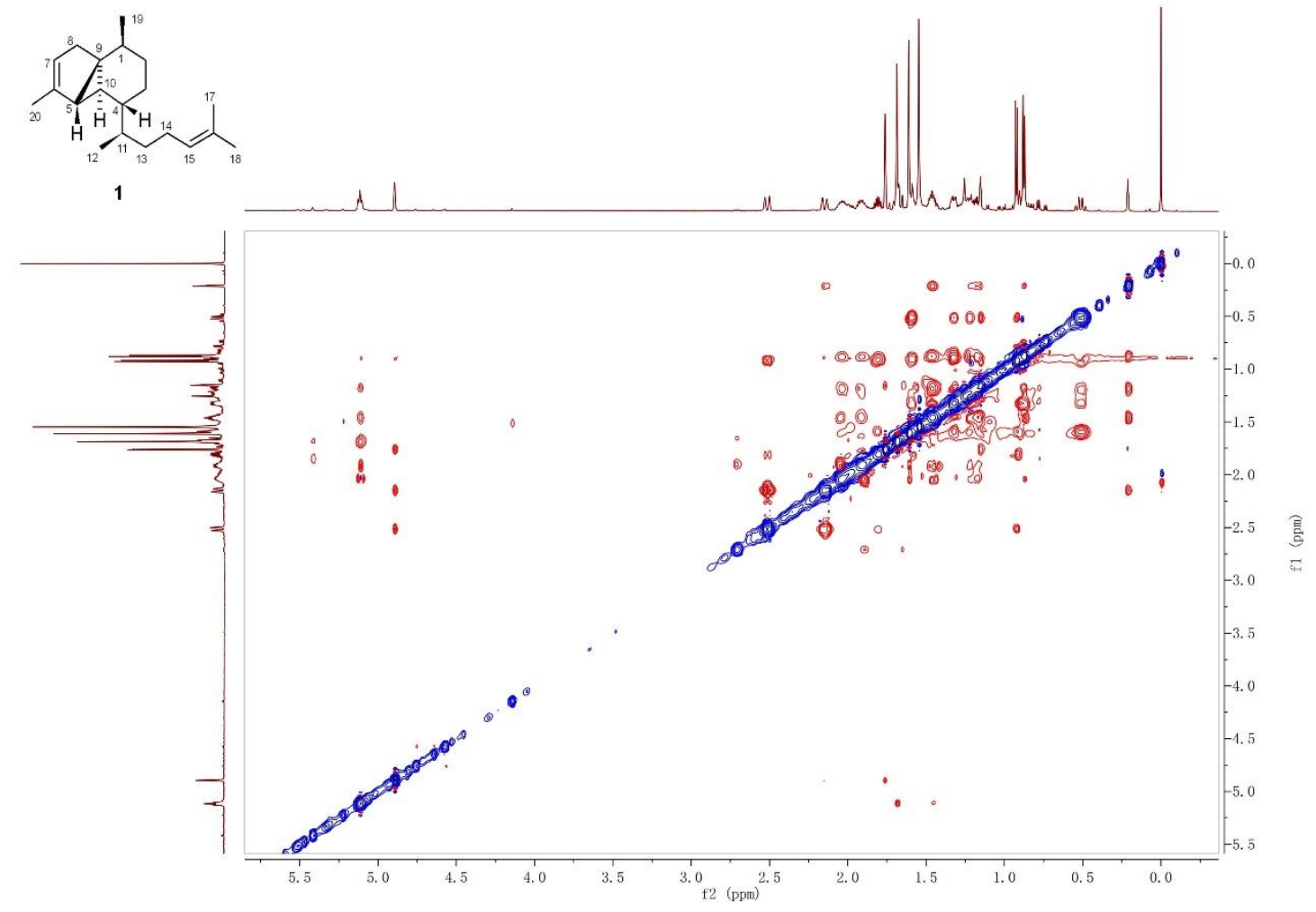


Figure S7. HR-ESI-MS spectrum of 1

Qualitative Analysis Report

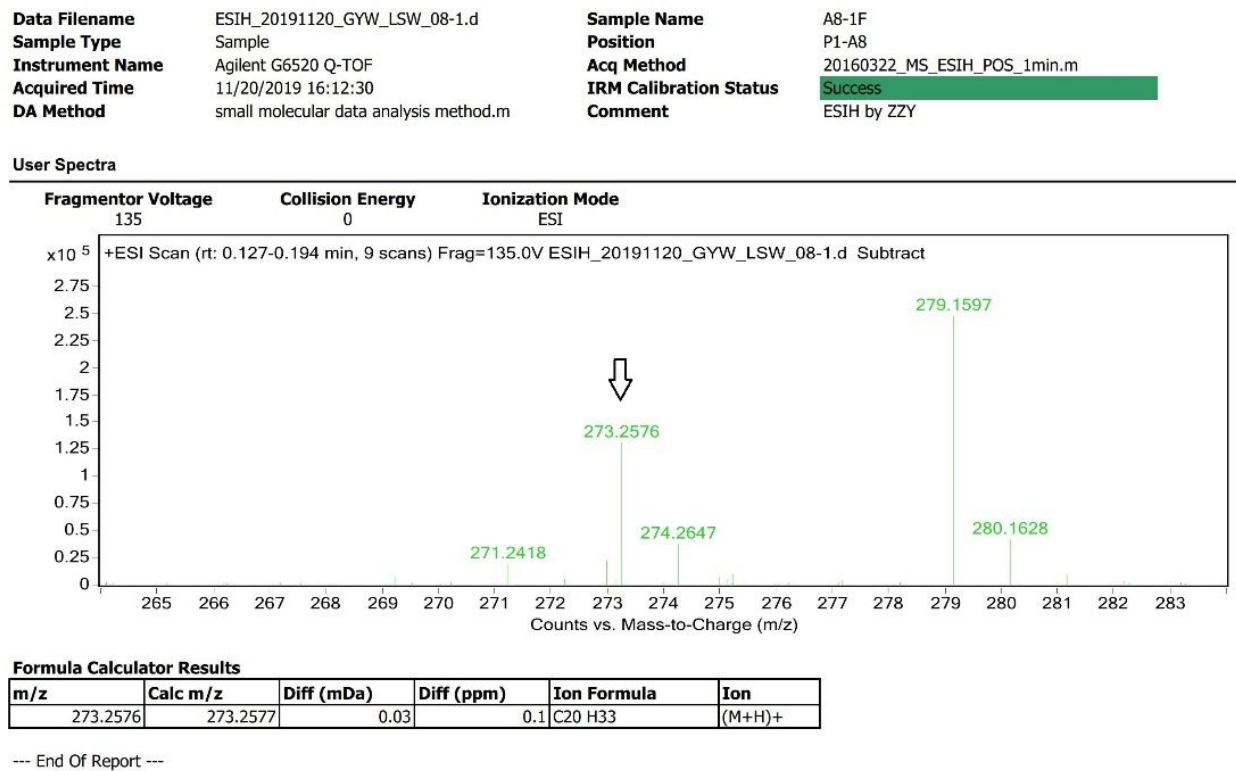

F Agilent Technologies

Page 1 of 1

Printed at: $16: 14$ on: 11/20/2019

Figure S8. IR spectrum of 1

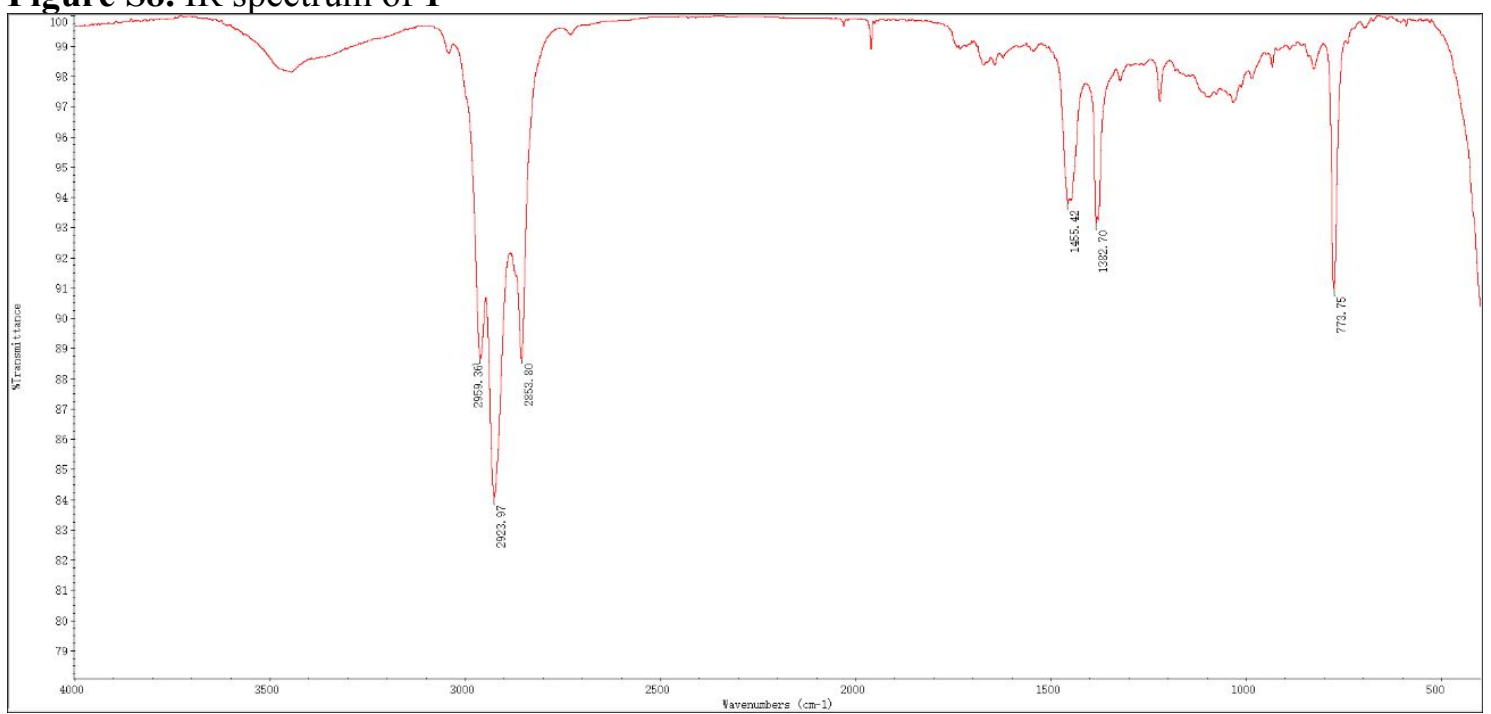


Figure S9. ${ }^{1} \mathrm{H}$ NMR spectrum of $2\left(500 \mathrm{MHz}, \mathrm{CDCl}_{3}\right)$

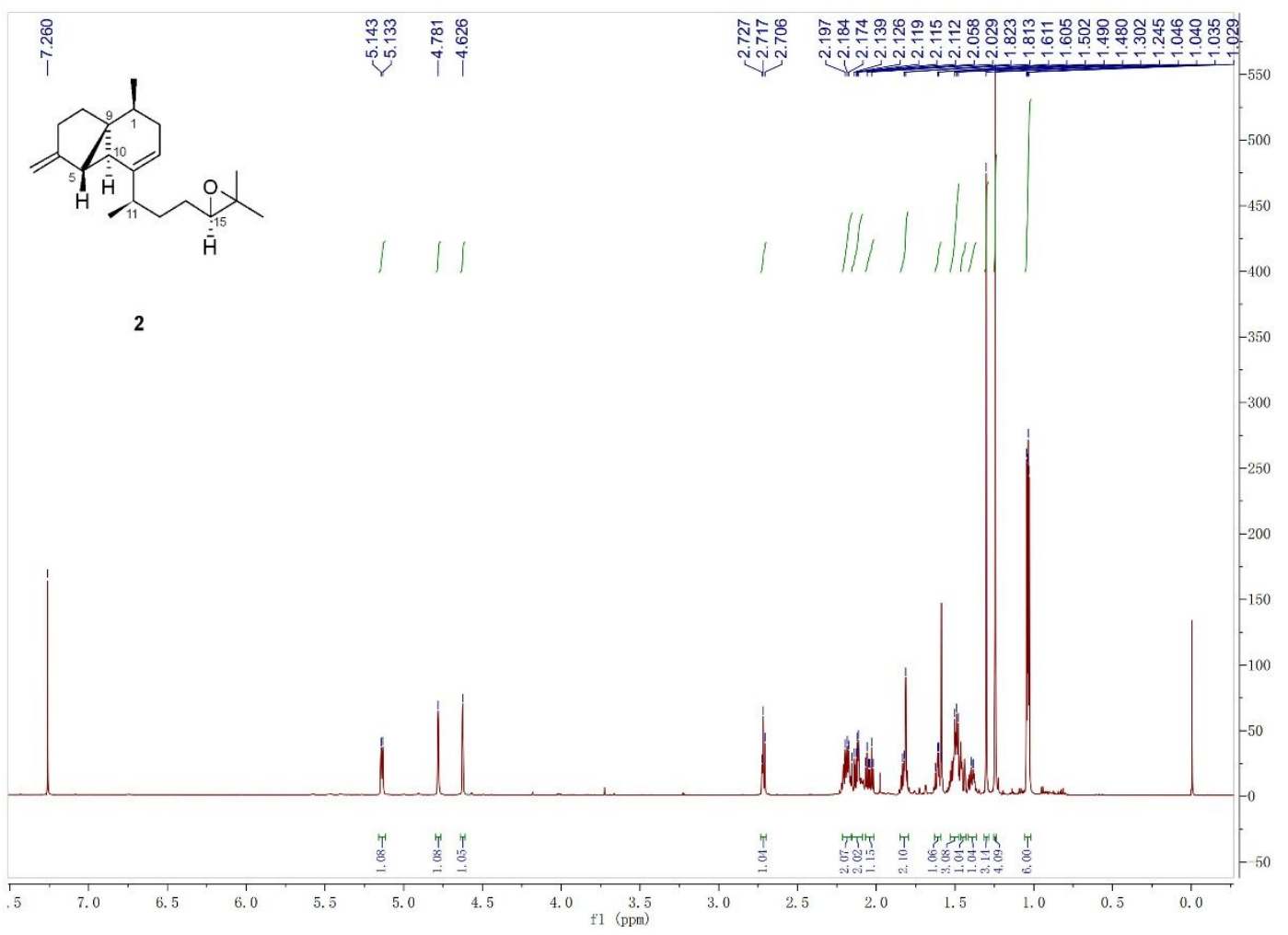

Figure S10. ${ }^{13} \mathrm{C}\left\{{ }^{1} \mathrm{H}\right\}$ NMR spectrum of $2\left(125 \mathrm{MHz}, \mathrm{CDCl}_{3}\right)$

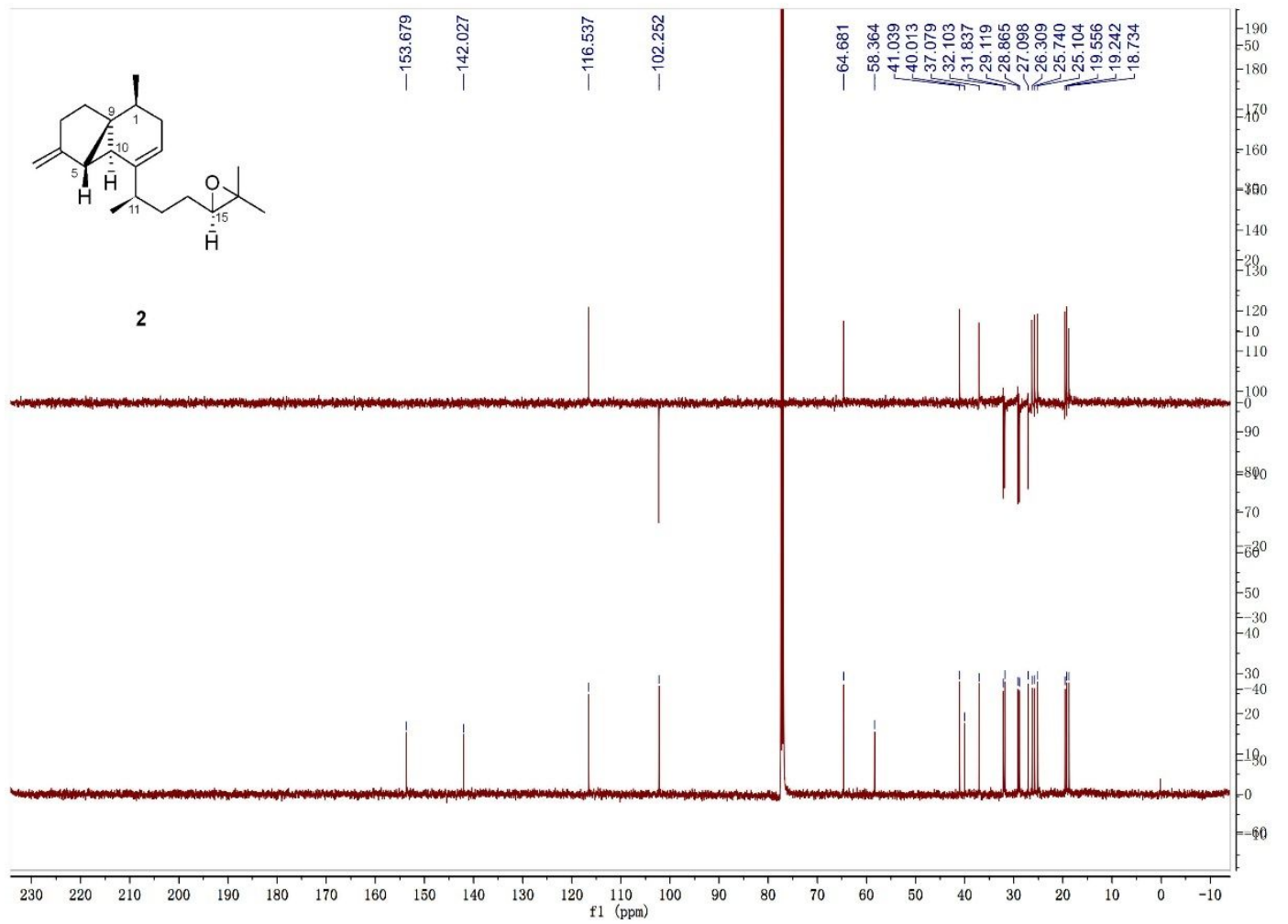


Figure S11. HSQC spectrum of $2\left(500 \mathrm{MHz}, \mathrm{CDCl}_{3}\right)$
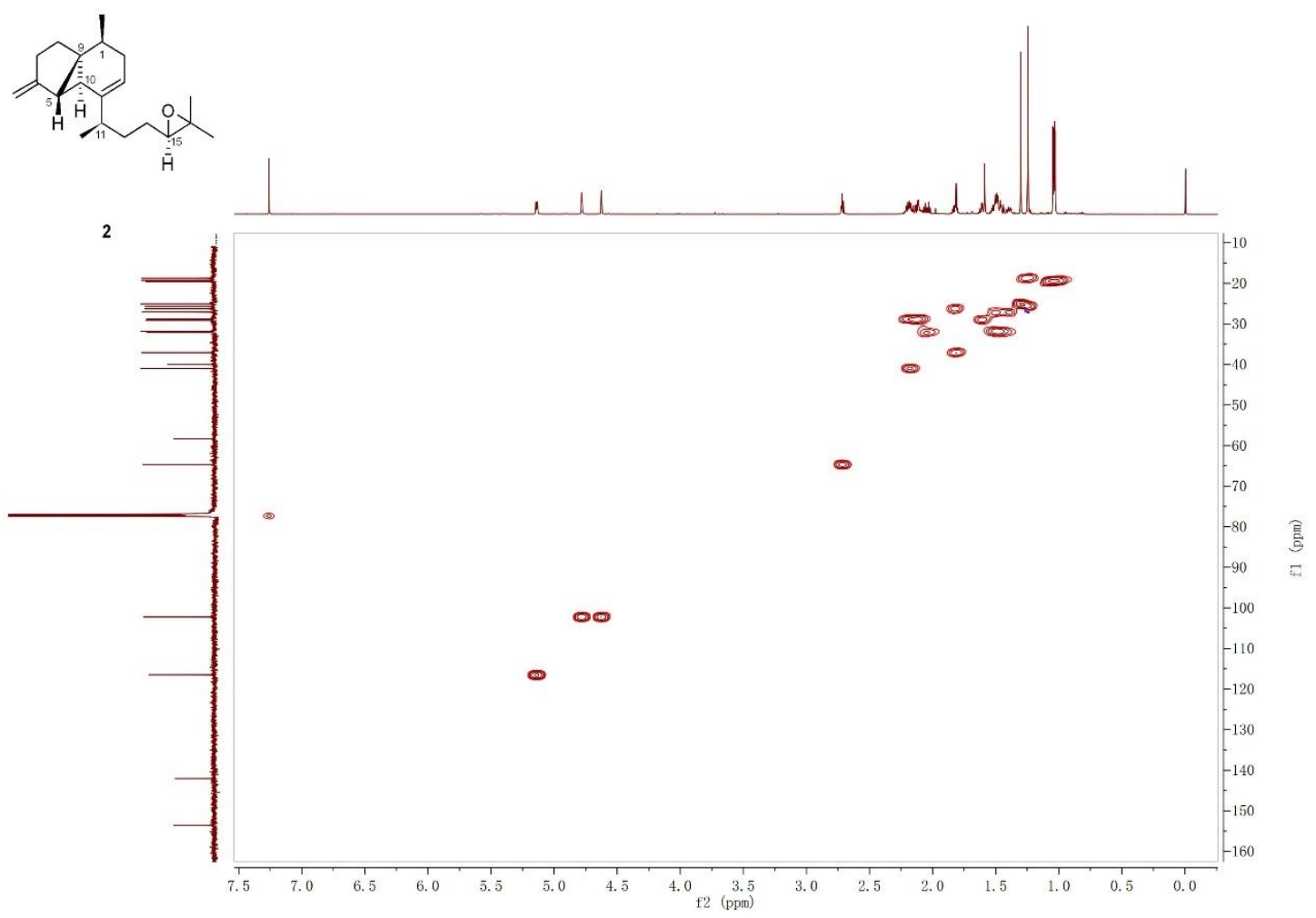

Figure S12. HMBC spectrum of $2\left(500 \mathrm{MHz}, \mathrm{CDCl}_{3}\right)$

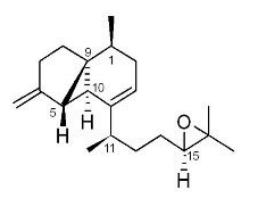

2

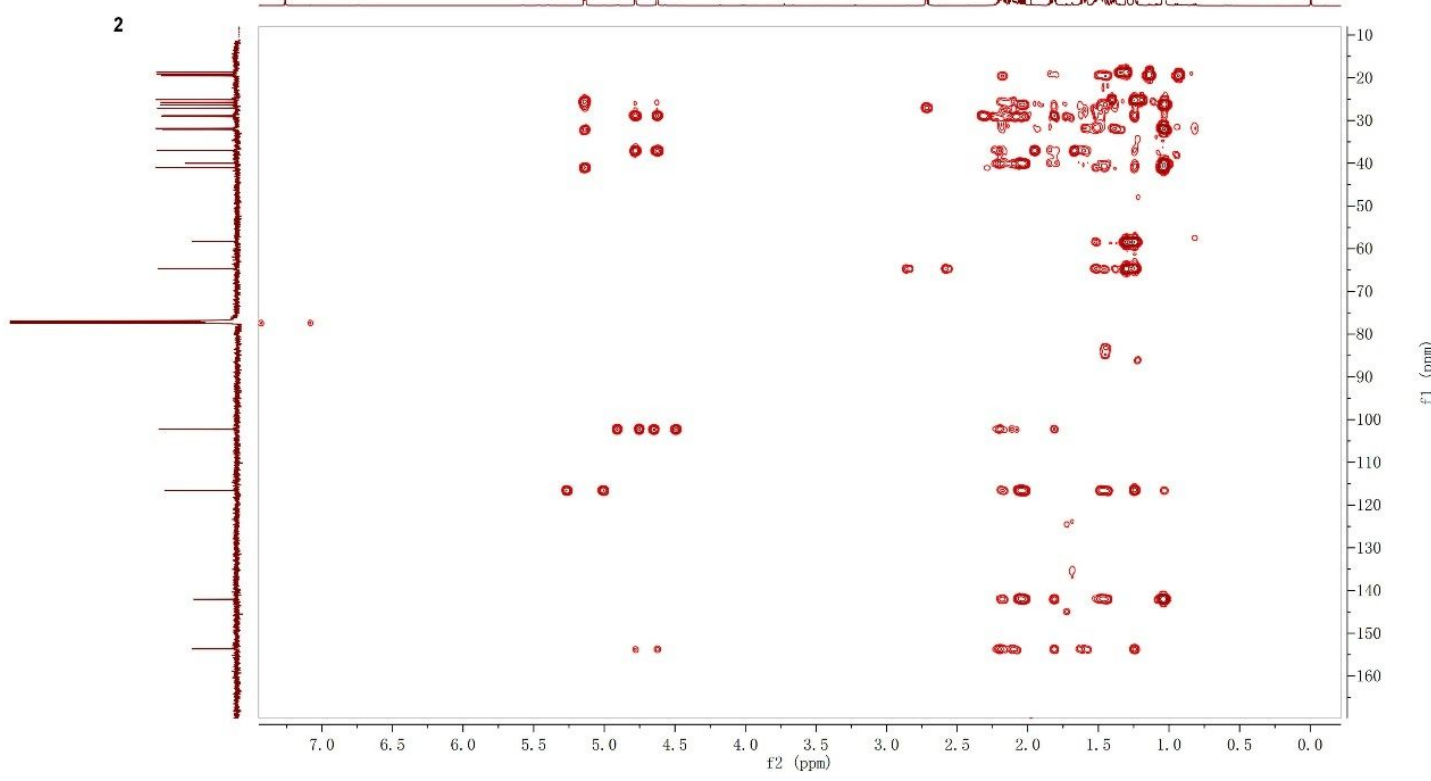

S11 
Figure S13. ${ }^{1} \mathrm{H}-{ }^{1} \mathrm{H}$ COSY spectrum of $2\left(500 \mathrm{MHz}, \mathrm{CDCl}_{3}\right)$

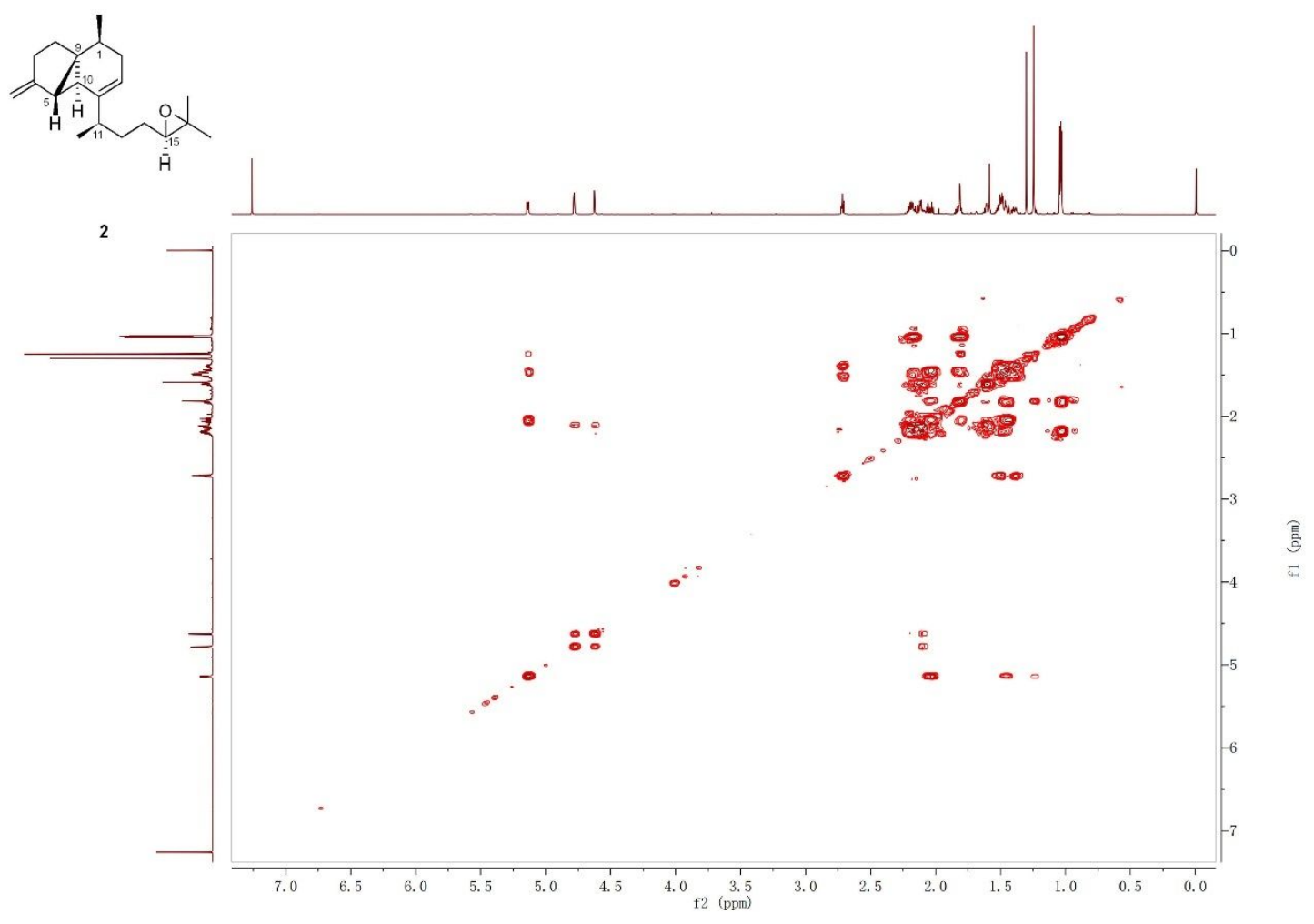

Figure S14. NOESY spectrum of $2\left(500 \mathrm{MHz}, \mathrm{CDCl}_{3}\right)$

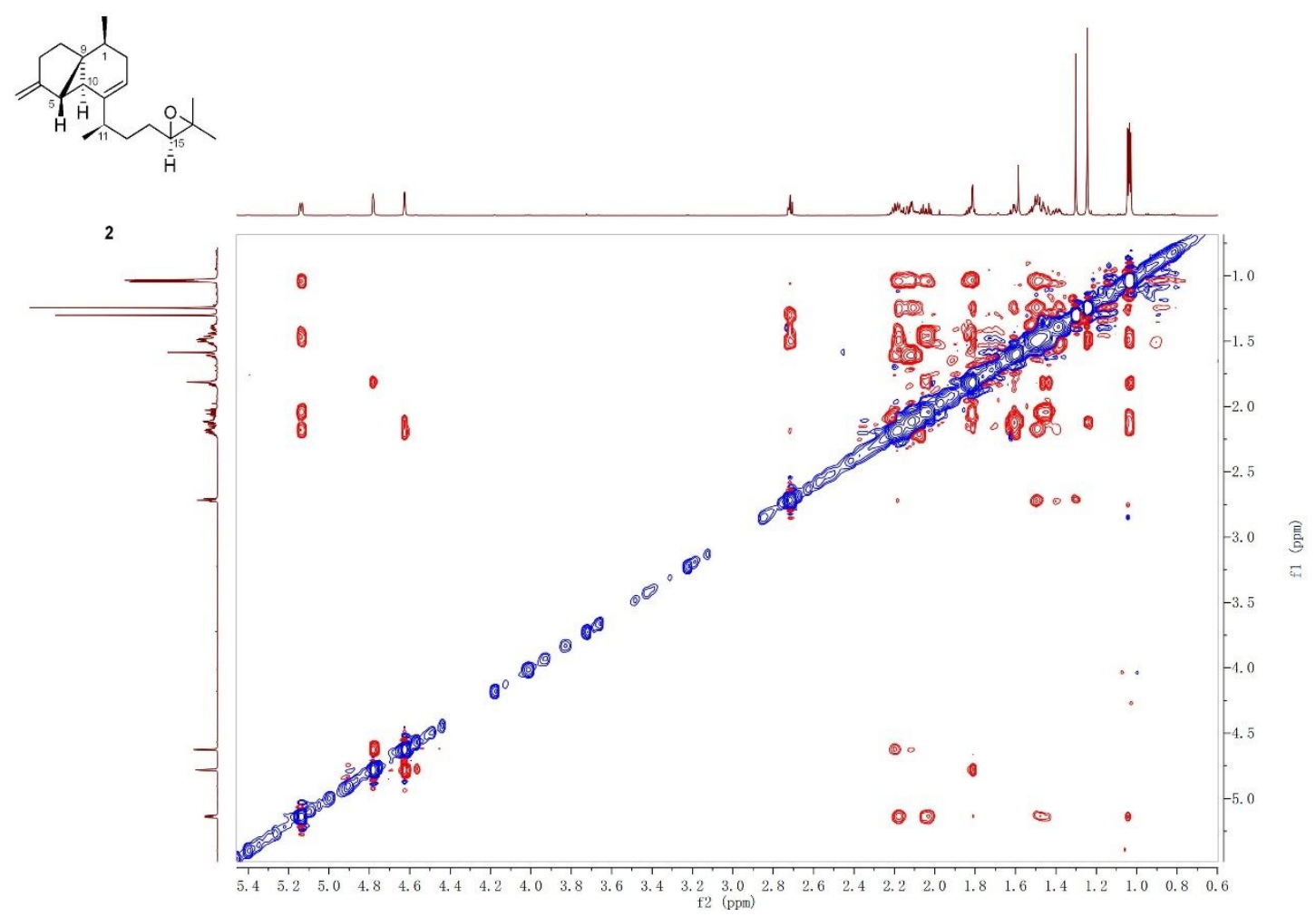


Figure S15. HR-ESI-MS spectrum of 2

Qualitative Analysis Report

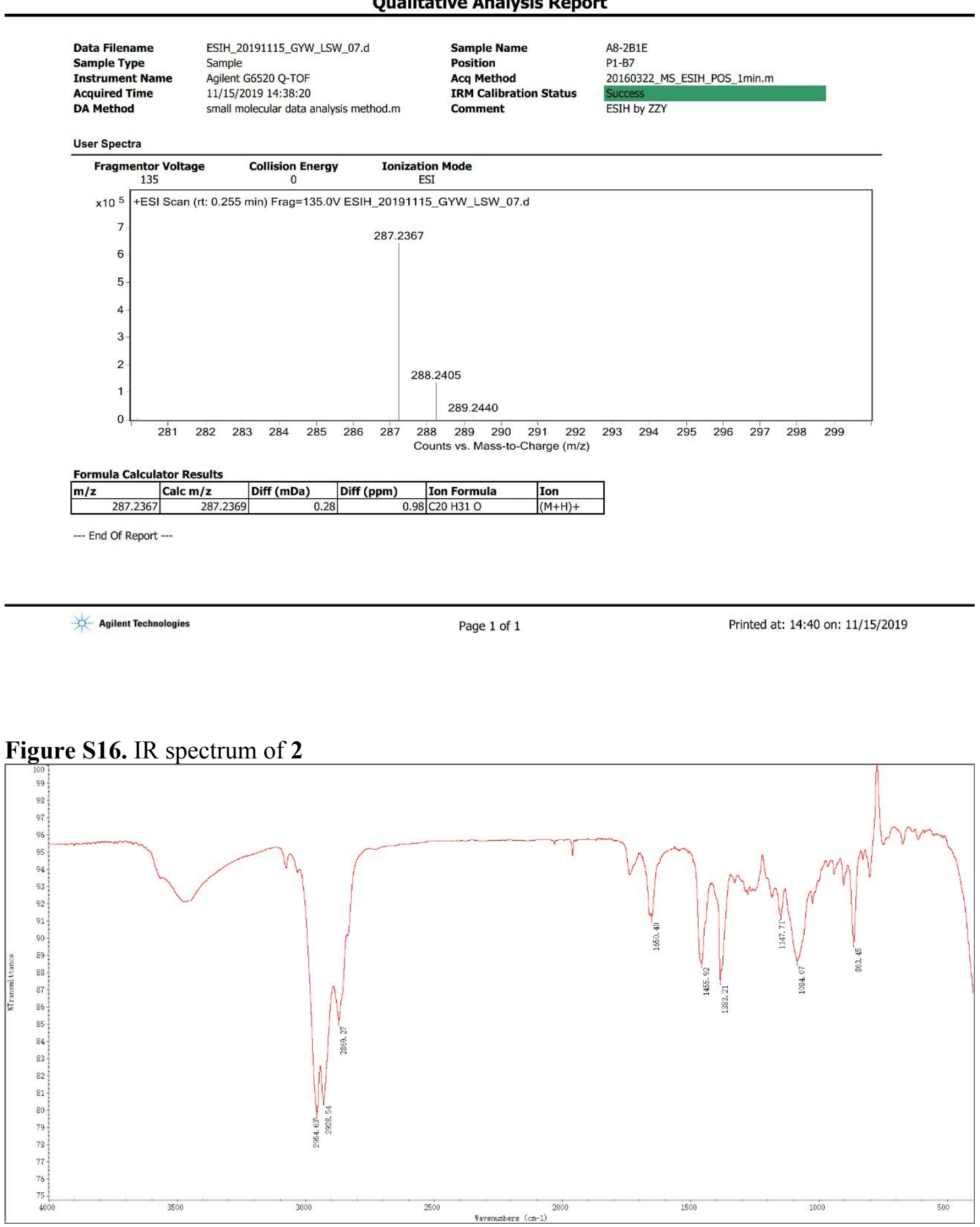


Figure S17. ${ }^{1} \mathrm{H}$ NMR spectrum of $\mathbf{3}\left(500 \mathrm{MHz}, \mathrm{CDCl}_{3}\right)$

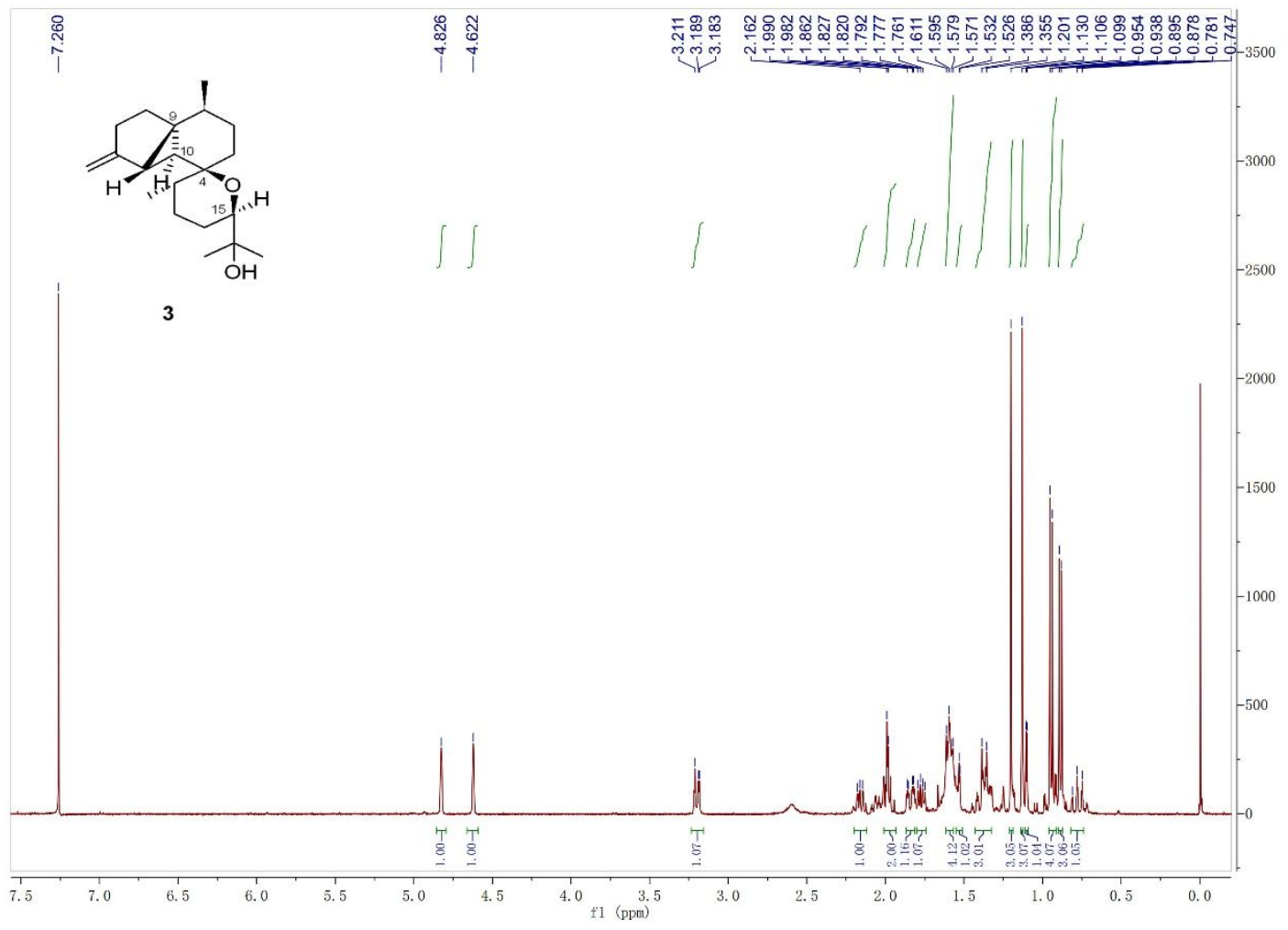

Figure S18. ${ }^{13} \mathrm{C}\left\{{ }^{1} \mathrm{H}\right\}$ NMR spectrum of $3\left(125 \mathrm{MHz}, \mathrm{CDCl}_{3}\right)$

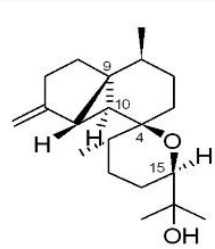

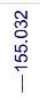

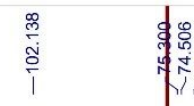

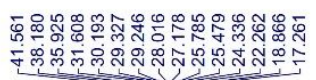

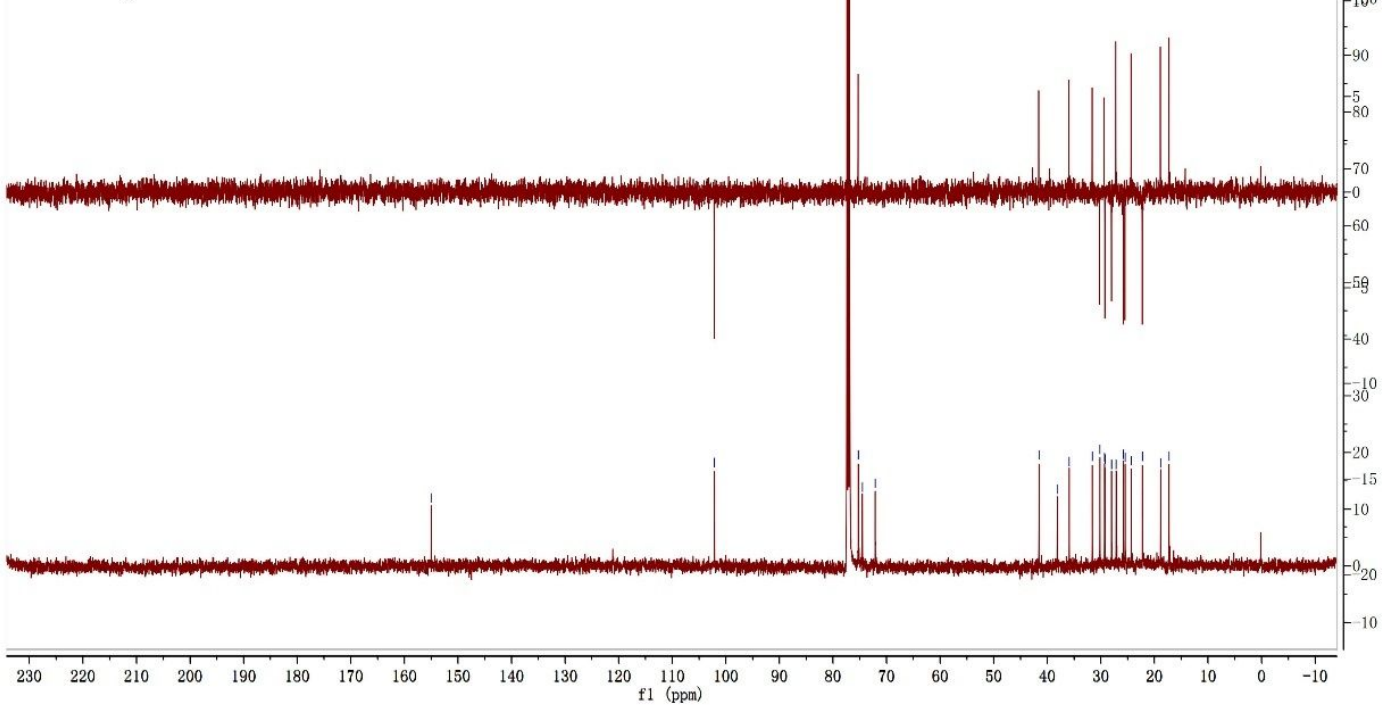


Figure S19. HSQC spectrum of $\mathbf{3}\left(500 \mathrm{MHz}, \mathrm{CDCl}_{3}\right)$

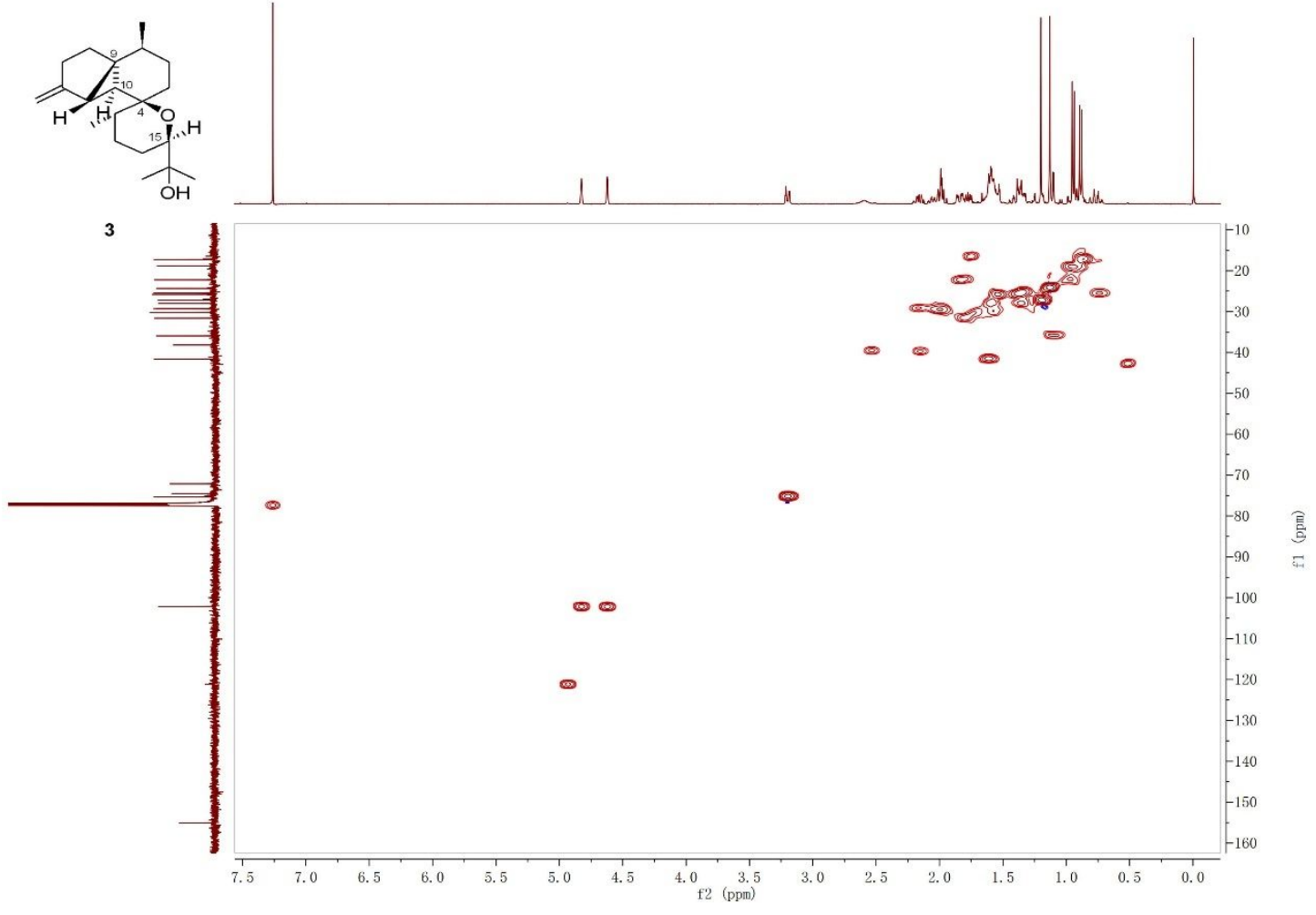

Figure S20. HMBC spectrum of $3\left(500 \mathrm{MHz}, \mathrm{CDCl}_{3}\right)$

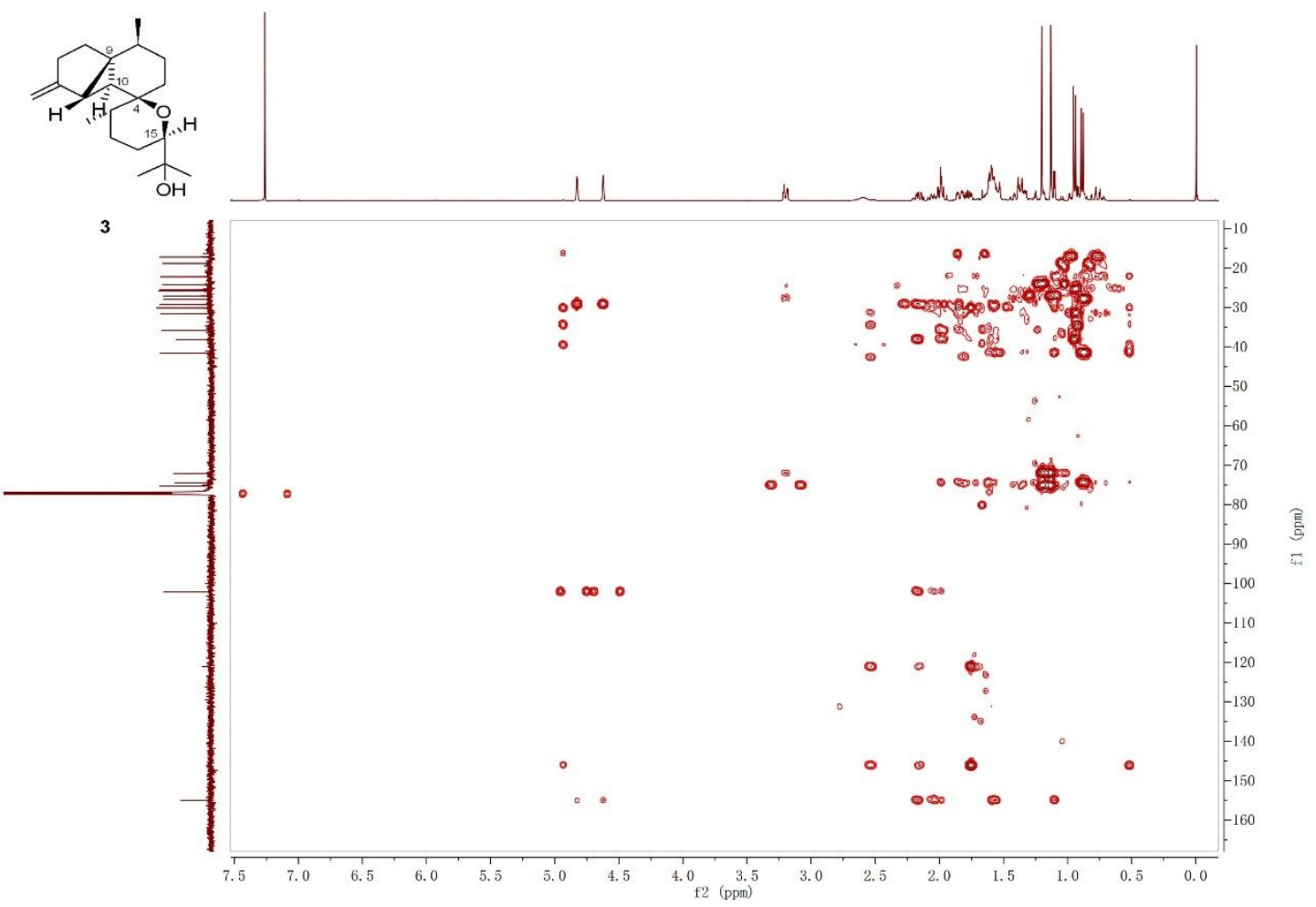


Figure S21. ${ }^{1} \mathrm{H}-{ }^{1} \mathrm{H}$ COSY spectrum of $3\left(500 \mathrm{MHz}, \mathrm{CDCl}_{3}\right)$

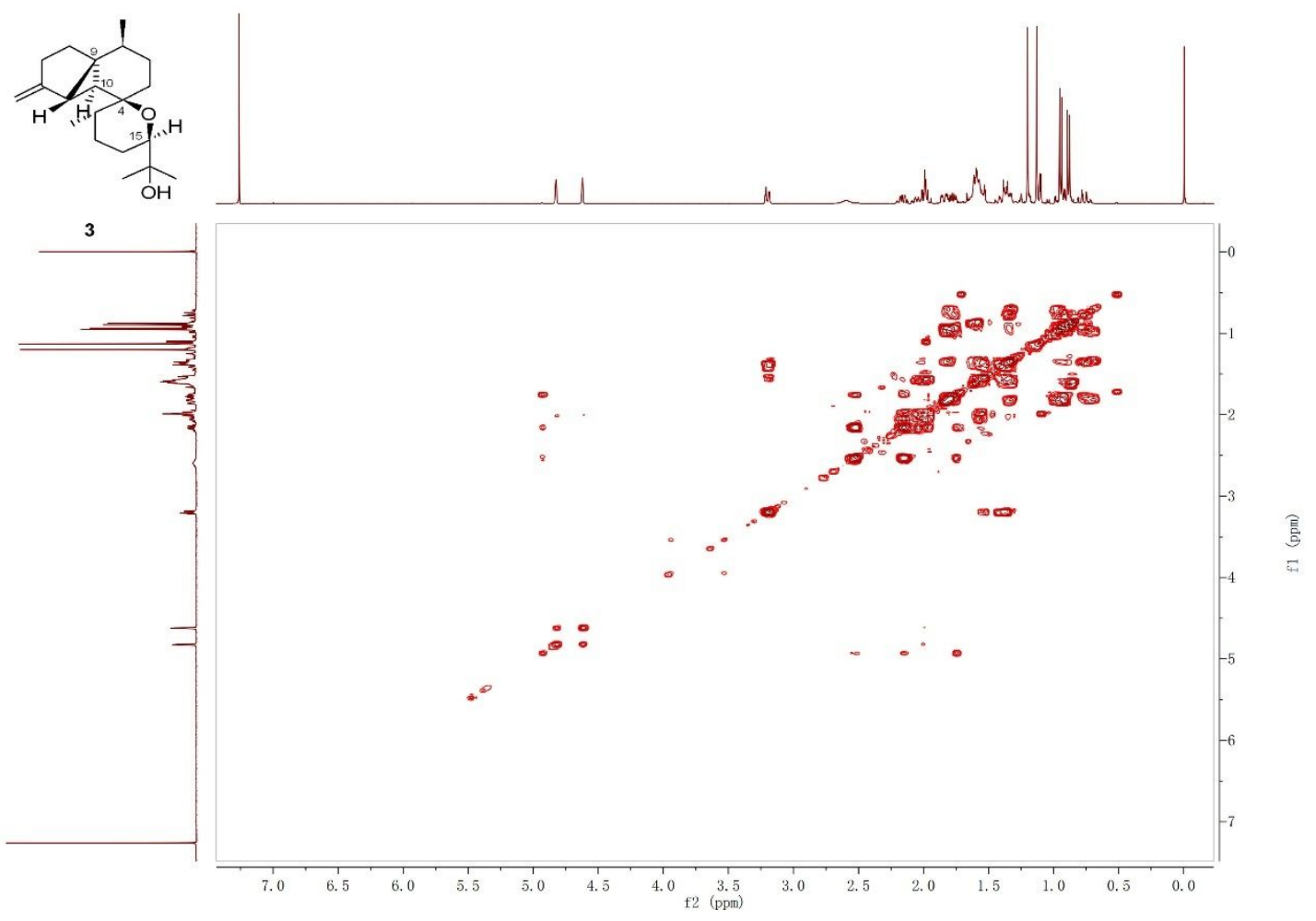

Figure S22. NOESY spectrum of $3\left(500 \mathrm{MHz}, \mathrm{CDCl}_{3}\right)$

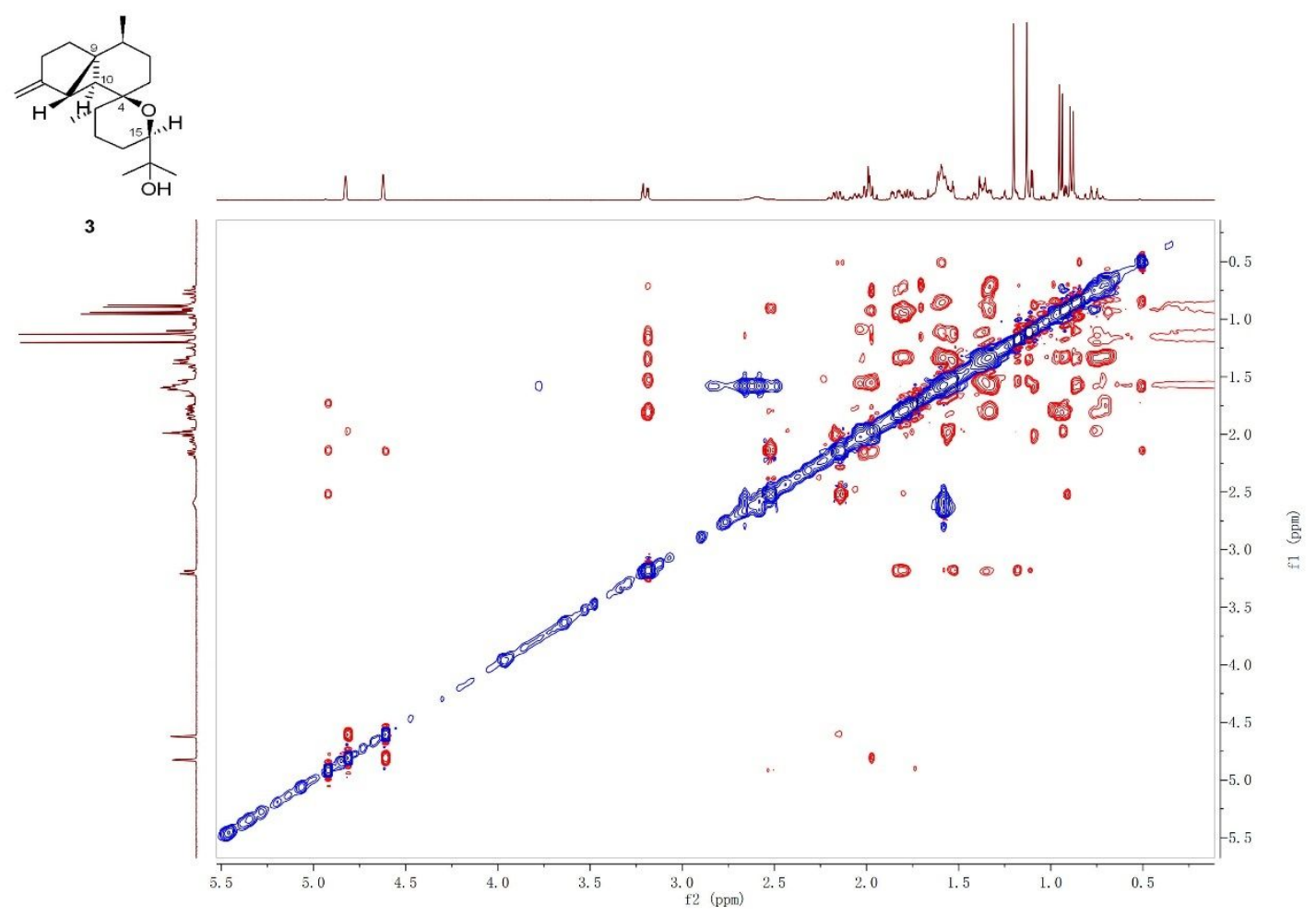


Figure S23. HR-ESI-MS spectrum of $\mathbf{3}$

Qualitative Analysis Report

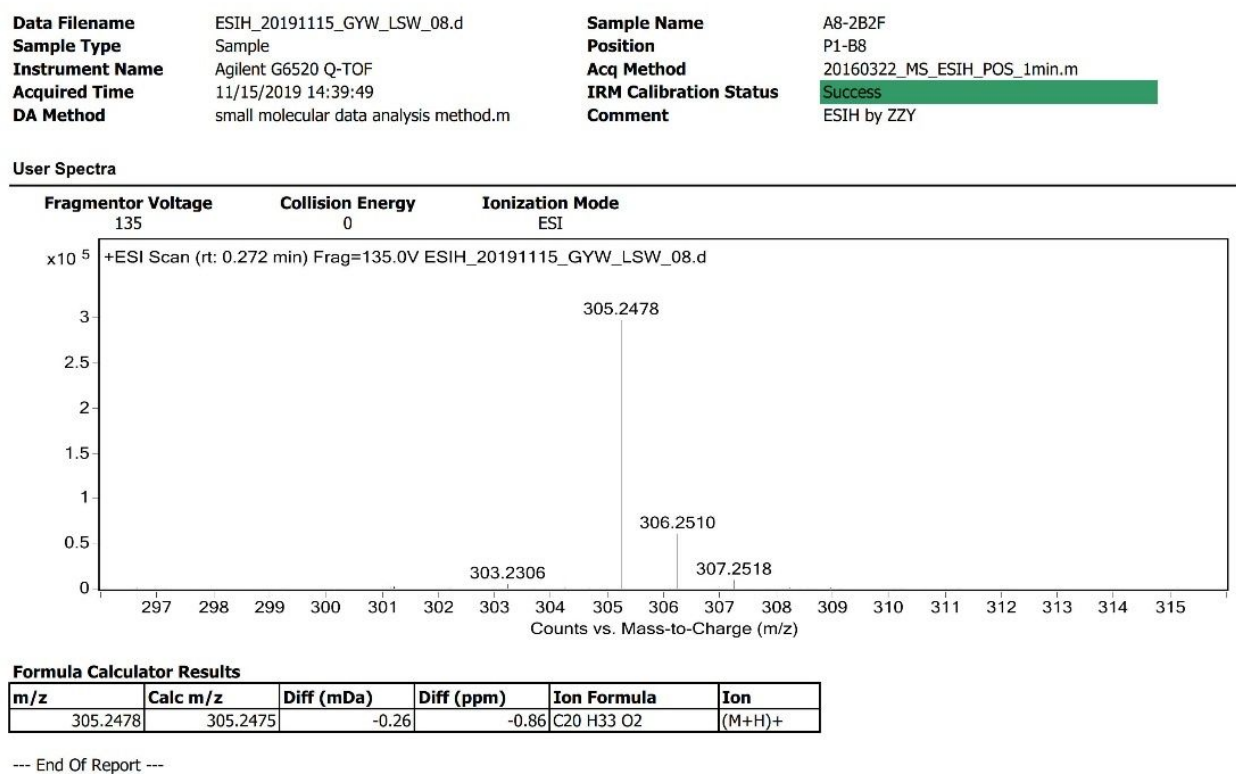

F Agilent Technologies

Page 1 of 1

Printed at: $14: 41$ on: 11/15/2019

Figure S24. IR spectrum of 3

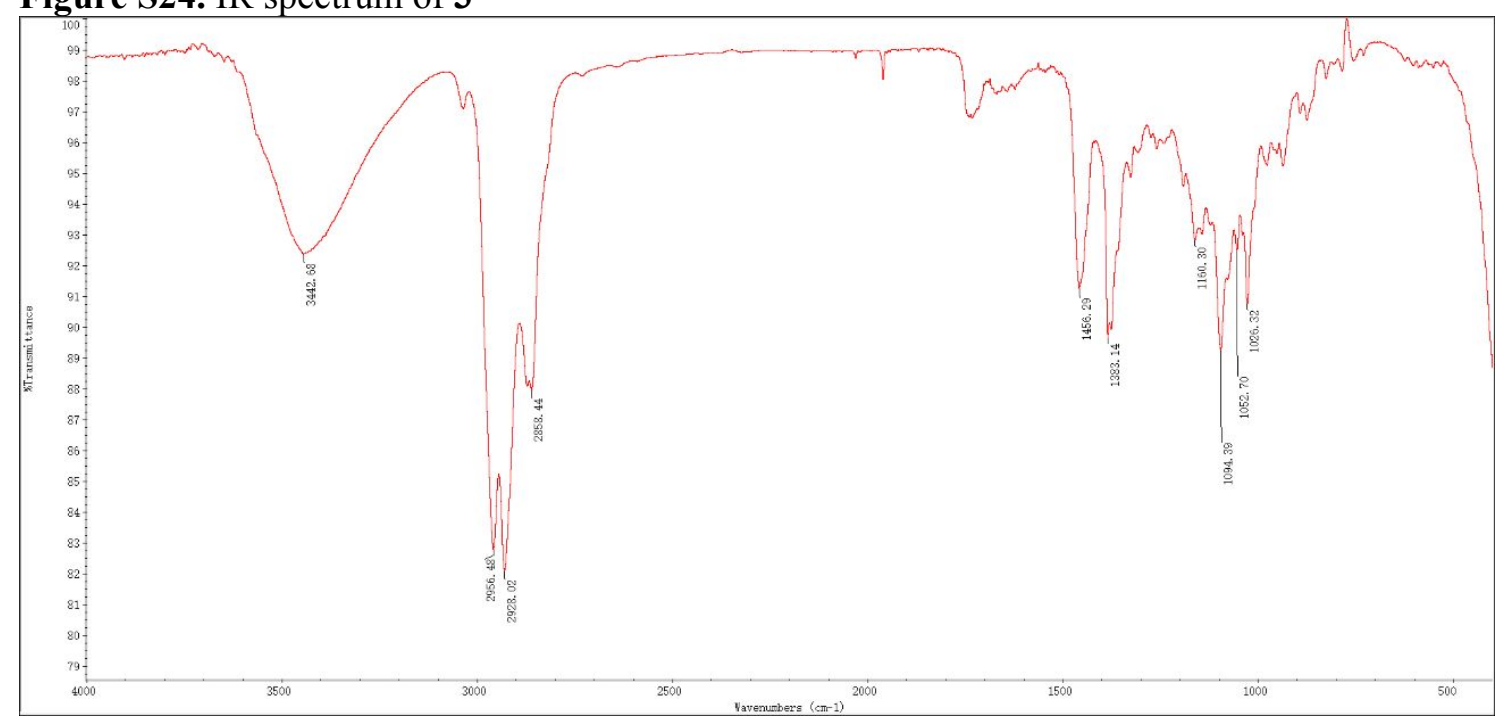


Figure S25. ${ }^{1} \mathrm{H}$ NMR spectrum of $4\left(500 \mathrm{MHz}, \mathrm{CDCl}_{3}\right)$

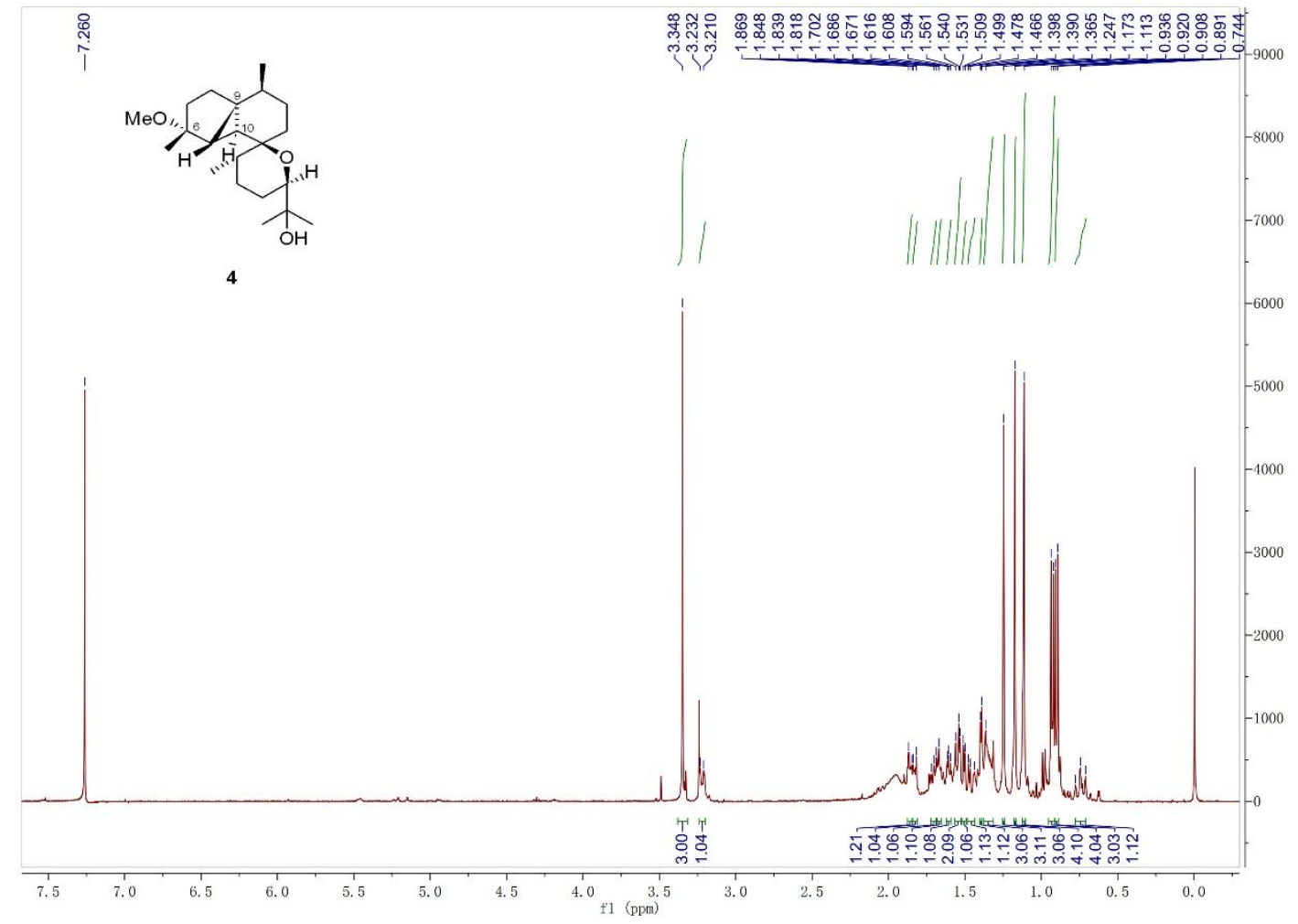

Figure S26. ${ }^{13} \mathrm{C}\left\{{ }^{1} \mathrm{H}\right\}$ NMR spectrum of $4\left(125 \mathrm{MHz}, \mathrm{CDCl}_{3}\right)$

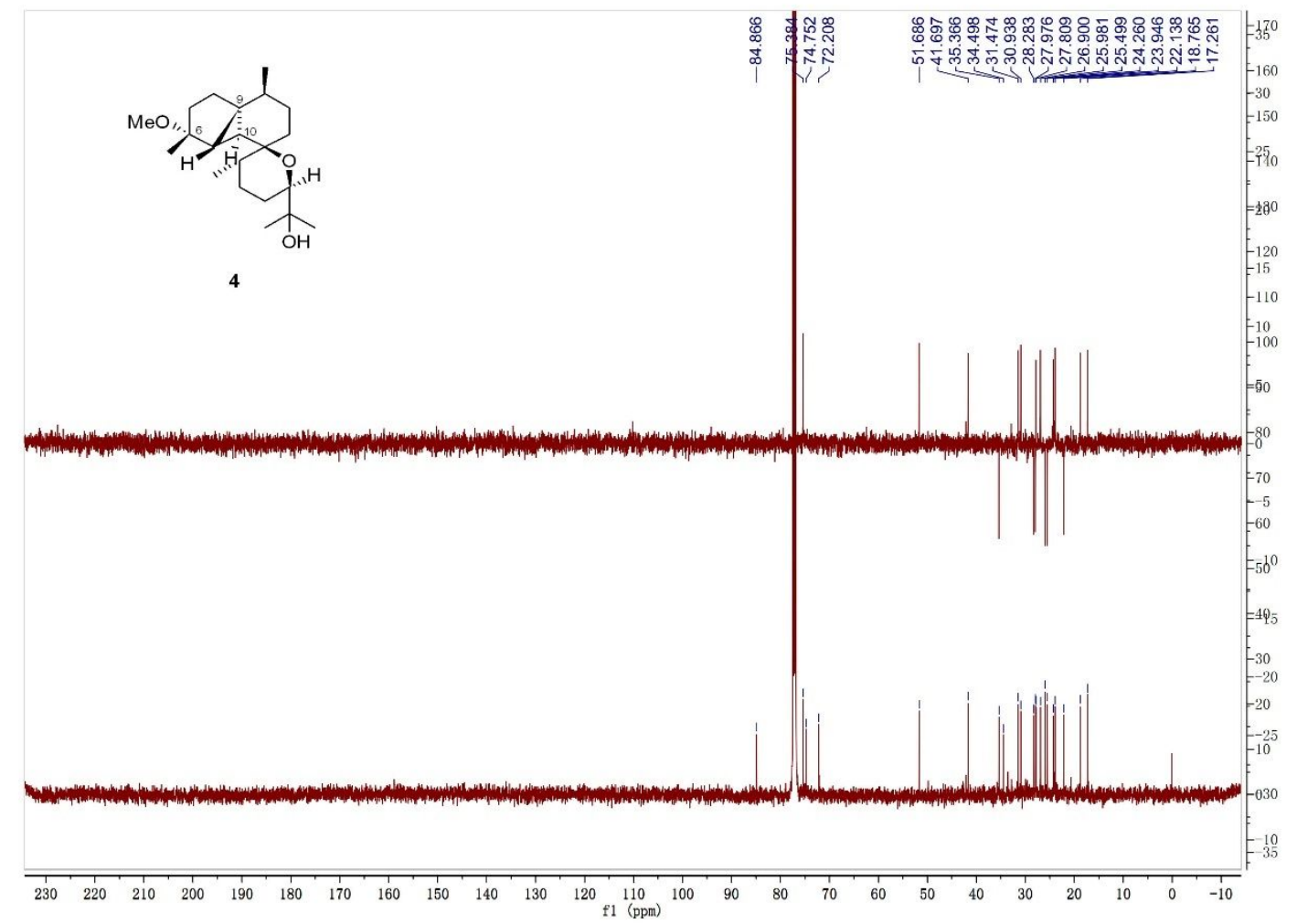


Figure S27. HSQC spectrum of $4\left(500 \mathrm{MHz}, \mathrm{CDCl}_{3}\right)$

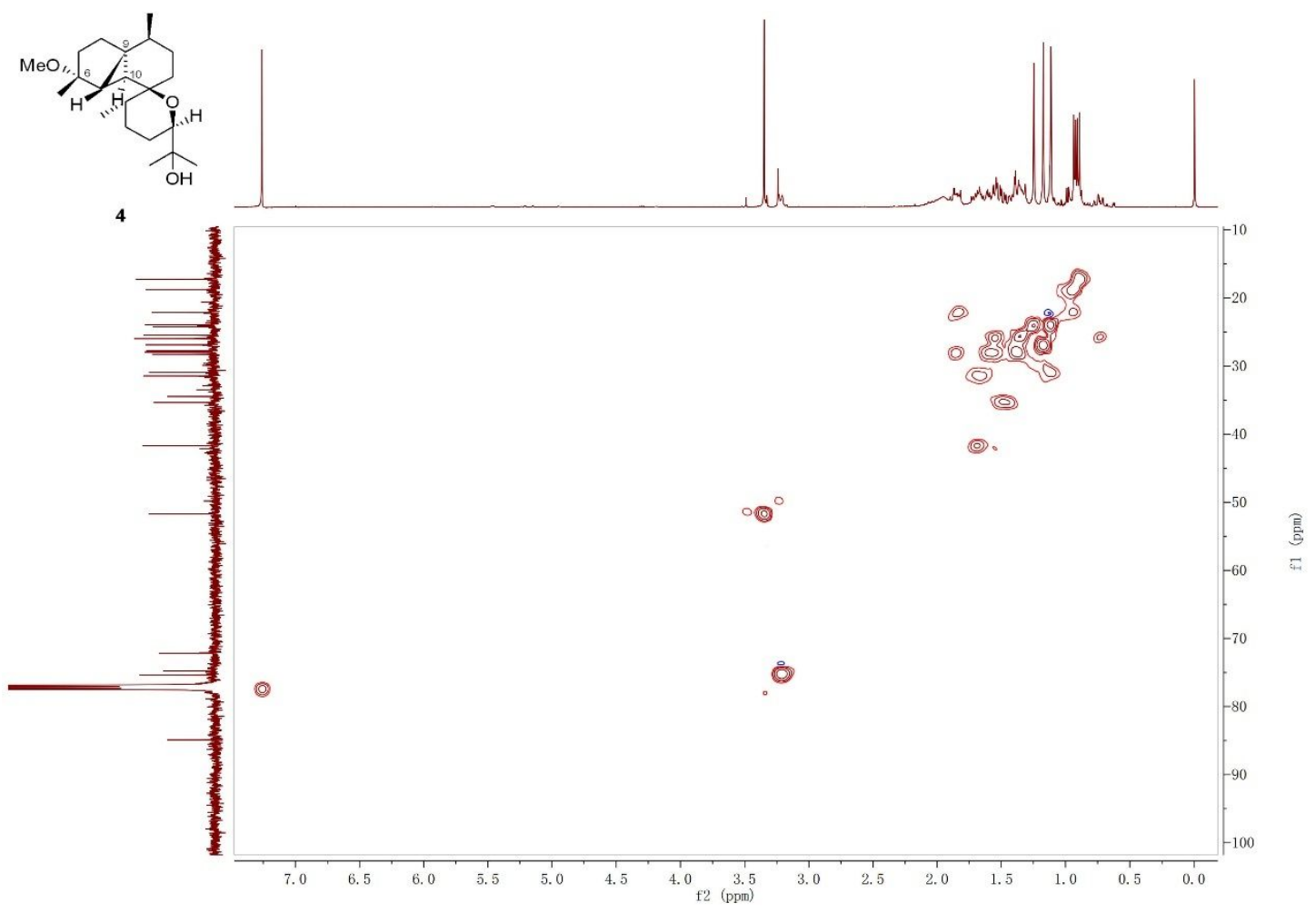

Figure S28. $\mathrm{HMBC}$ spectrum of $4\left(500 \mathrm{MHz}, \mathrm{CDCl}_{3}\right)$

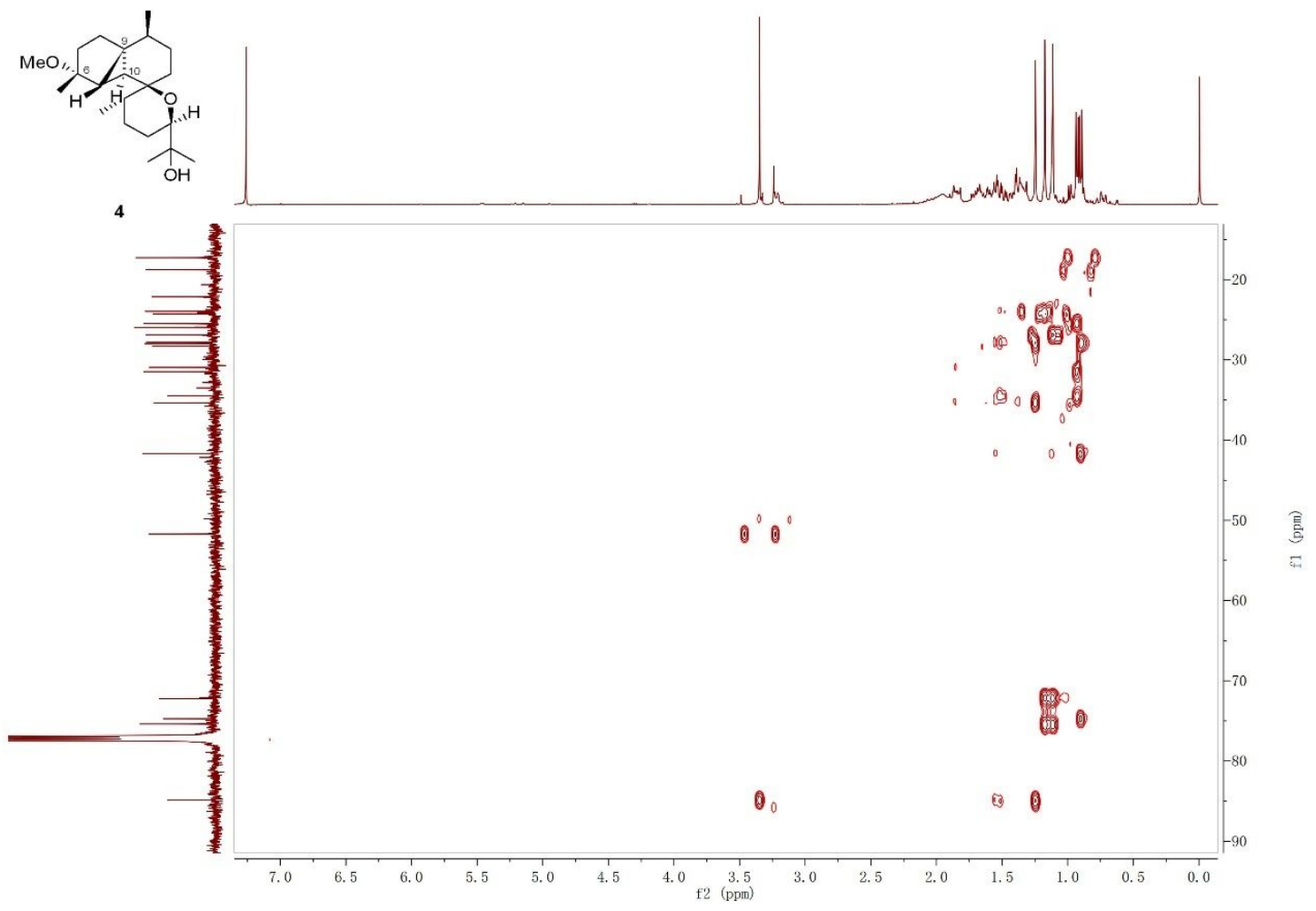

S19 
Figure S29. ${ }^{1} \mathrm{H}-{ }^{1} \mathrm{H}$ COSY spectrum of $4\left(500 \mathrm{MHz}, \mathrm{CDCl}_{3}\right)$

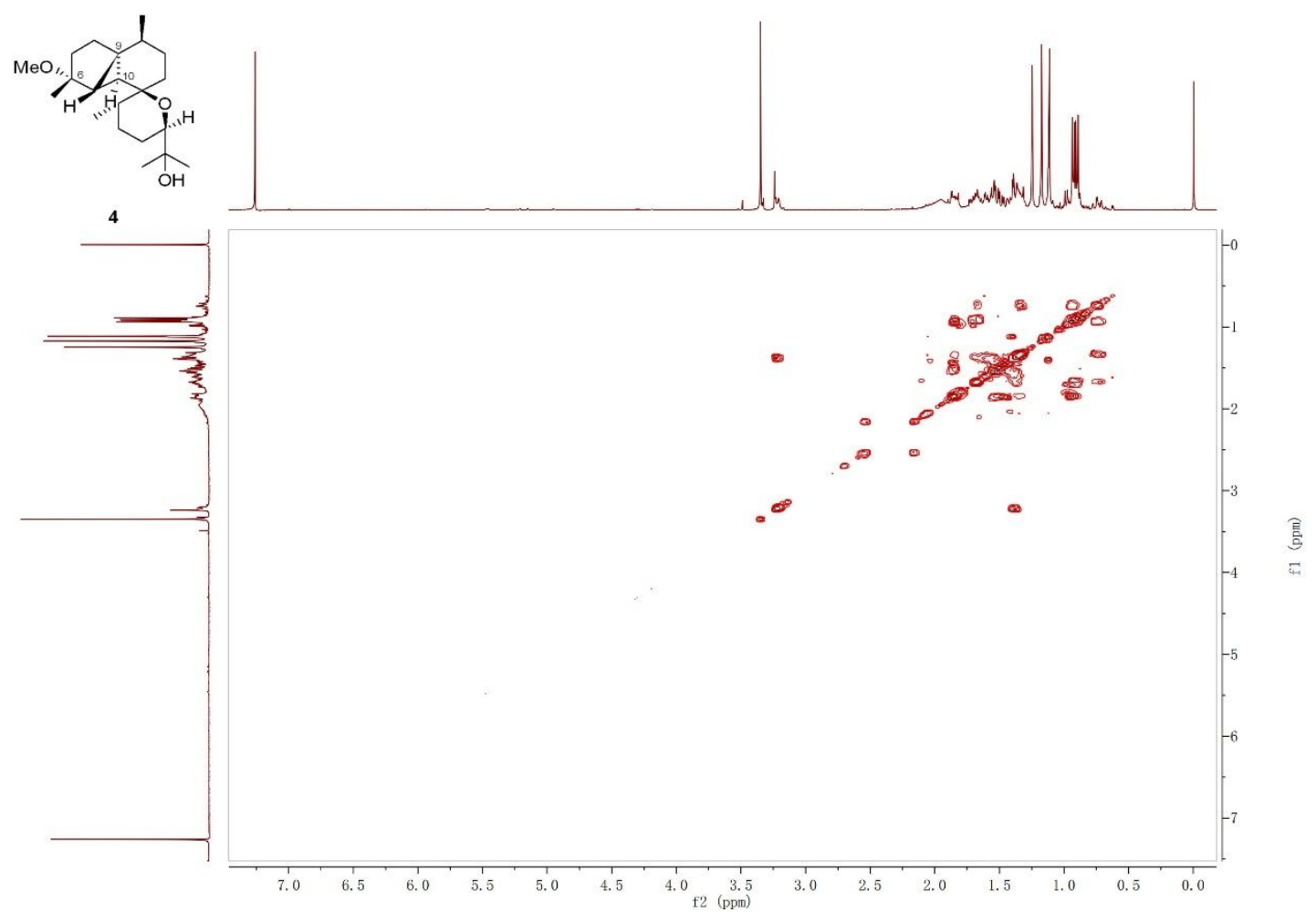

Figure S30. NOESY spectrum of $4\left(500 \mathrm{MHz}, \mathrm{CDCl}_{3}\right)$
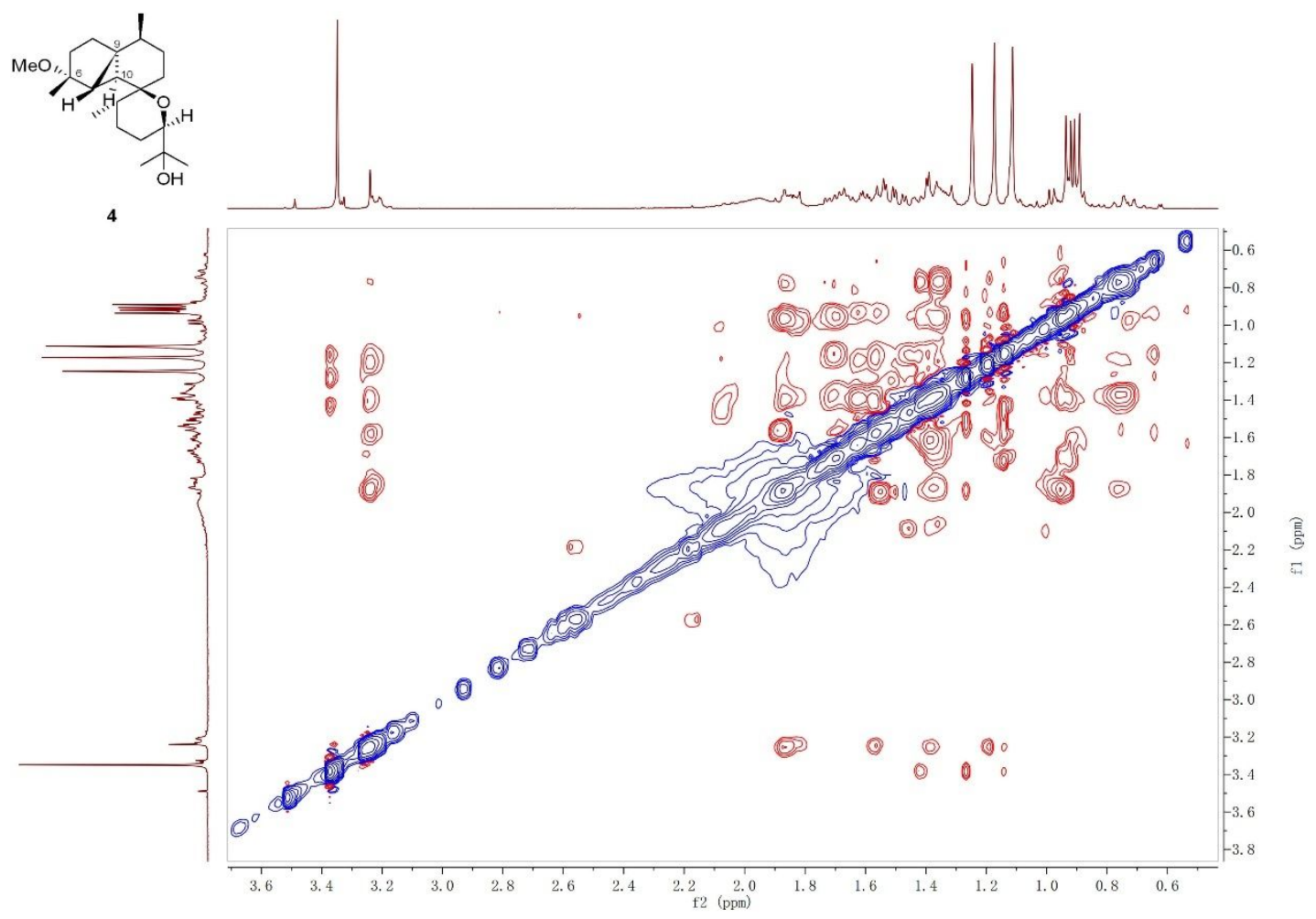
Figure S31. HR-ESI-MS spectrum of 4

Qualitative Analysis Report

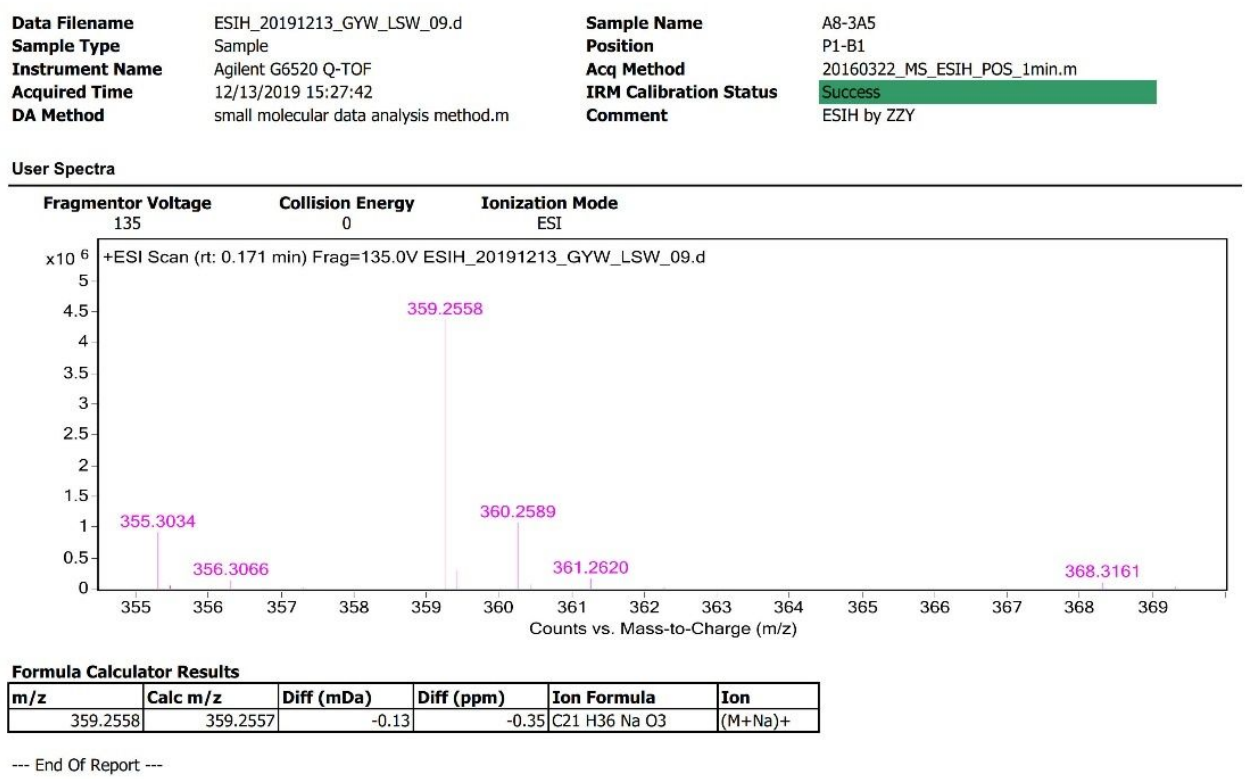

Agilent Technologies

Page 1 of 1

Printed at: $15: 41$ on: 12/13/2019

Figure S32. IR spectrum of 4

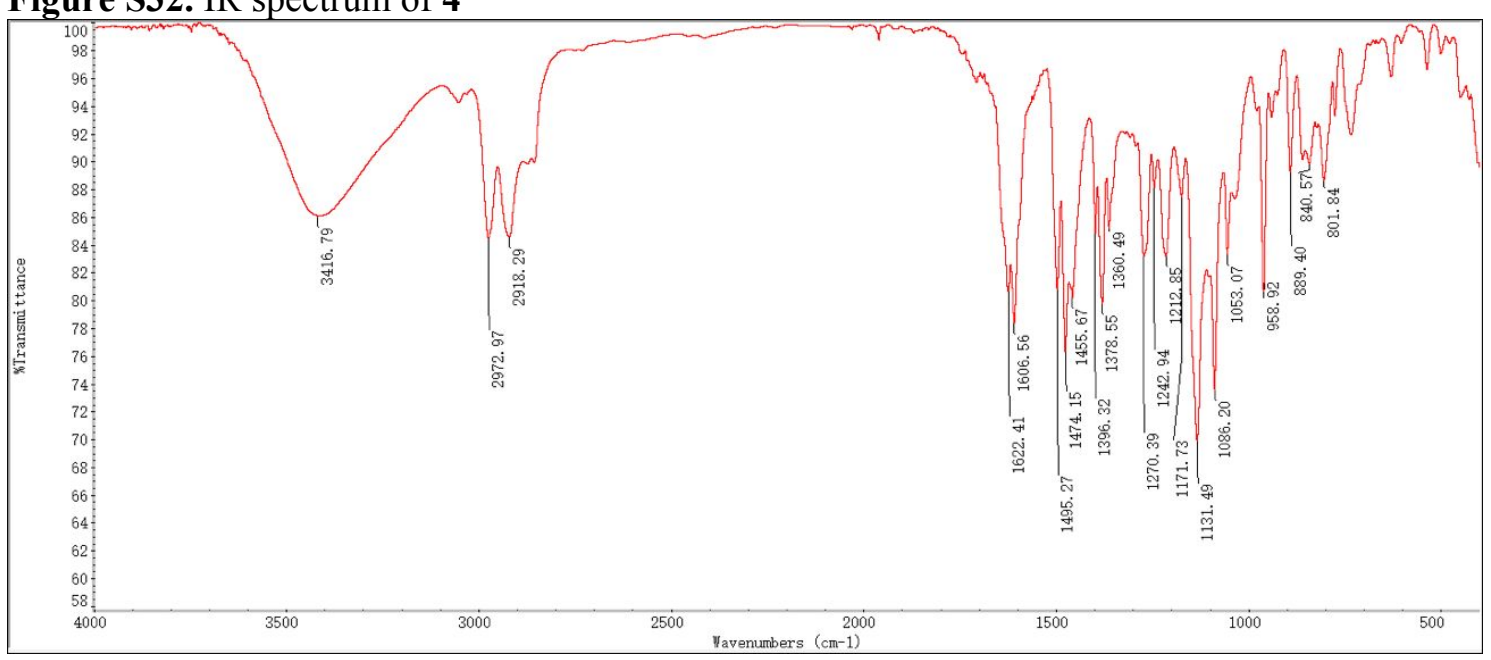


Figure S33. ${ }^{1} \mathrm{H}$ NMR spectrum of $5\left(500 \mathrm{MHz}, \mathrm{CDCl}_{3}\right)$

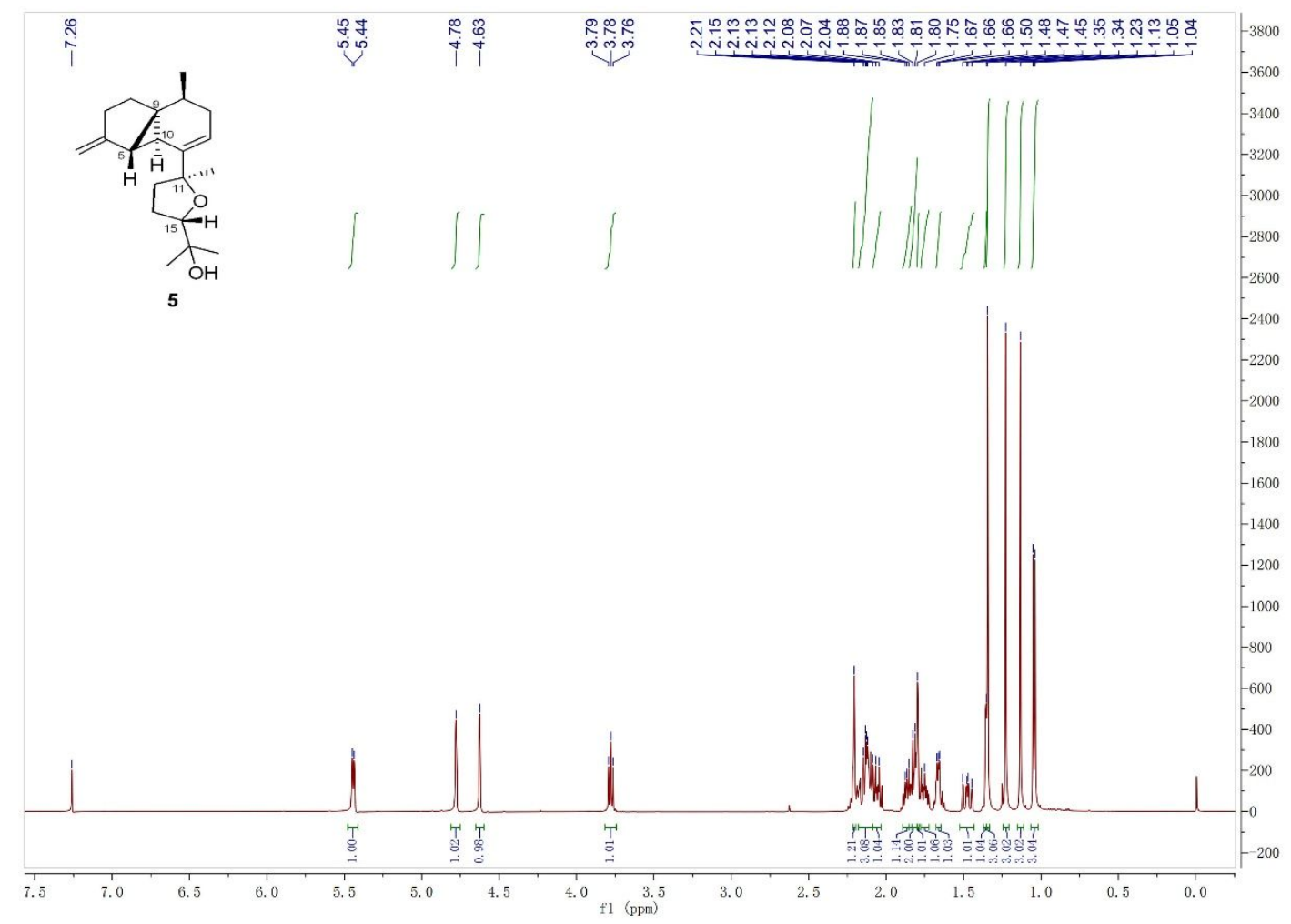

Figure S34. ${ }^{13} \mathrm{C}\left\{{ }^{1} \mathrm{H}\right\}$ NMR spectrum of $5\left(125 \mathrm{MHz}, \mathrm{CDCl}_{3}\right)$

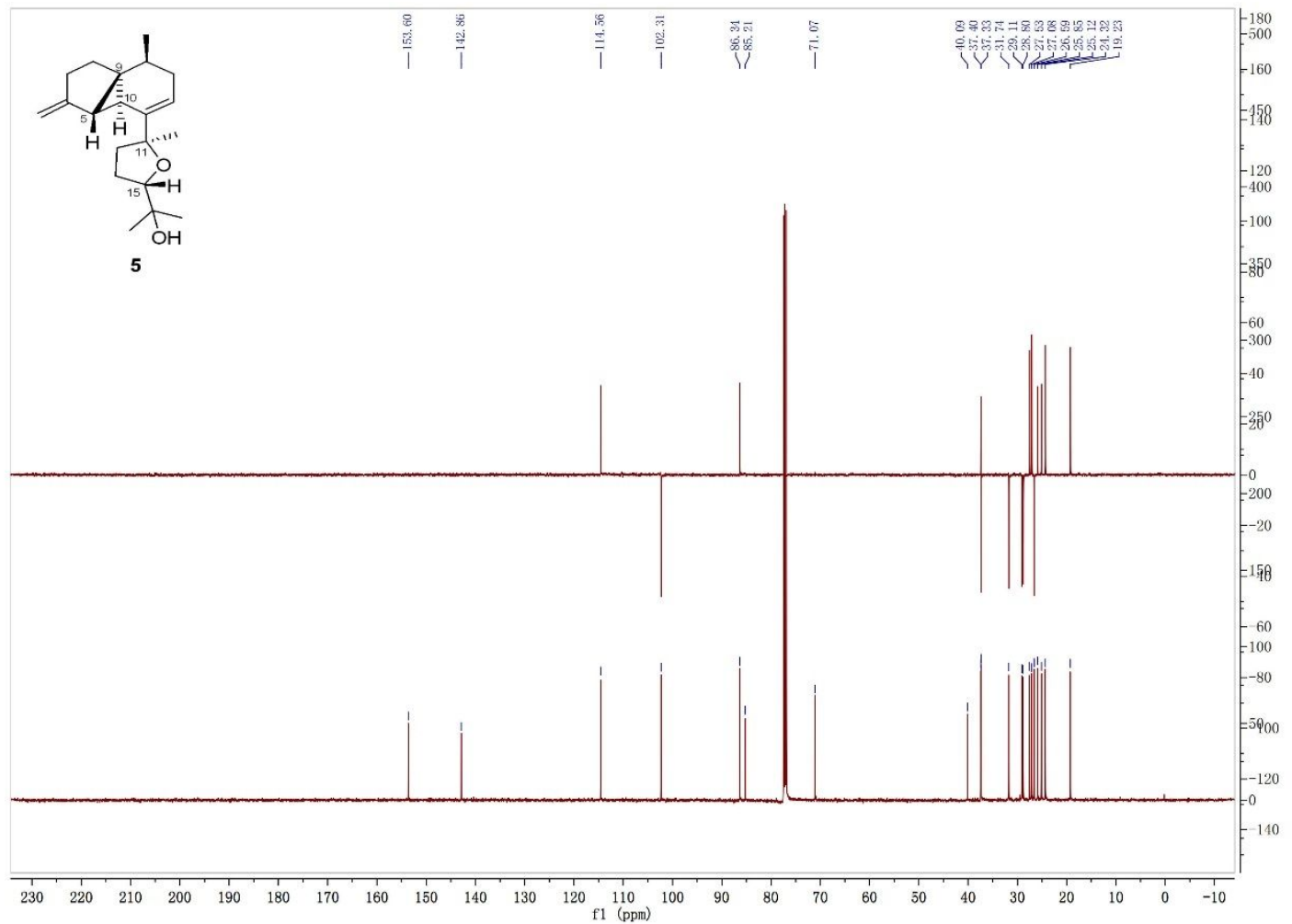


Figure S35. HSQC spectrum of $5\left(500 \mathrm{MHz}, \mathrm{CDCl}_{3}\right)$

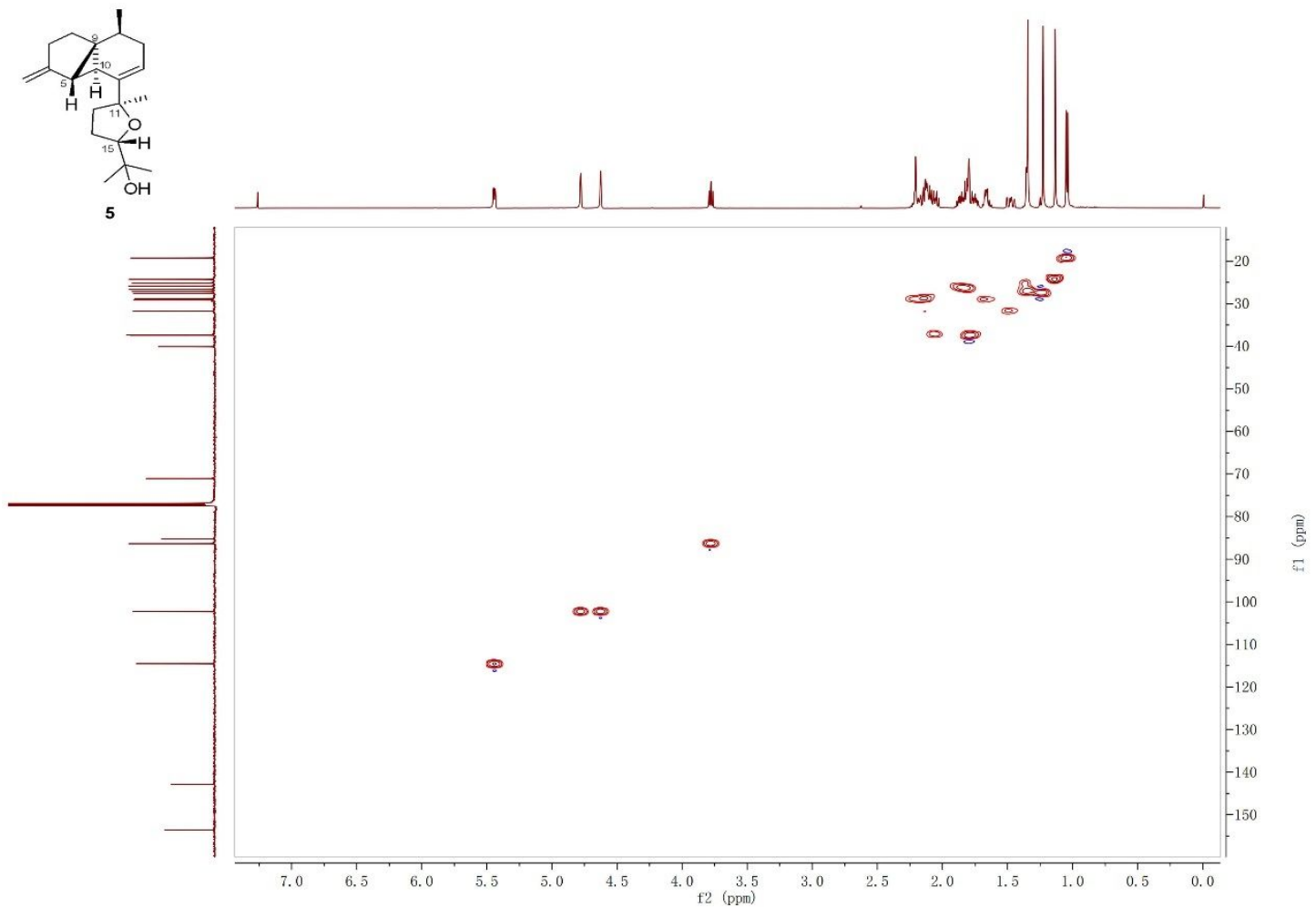

Figure S36. $\mathrm{HMBC}$ spectrum of $5\left(500 \mathrm{MHz}, \mathrm{CDCl}_{3}\right)$

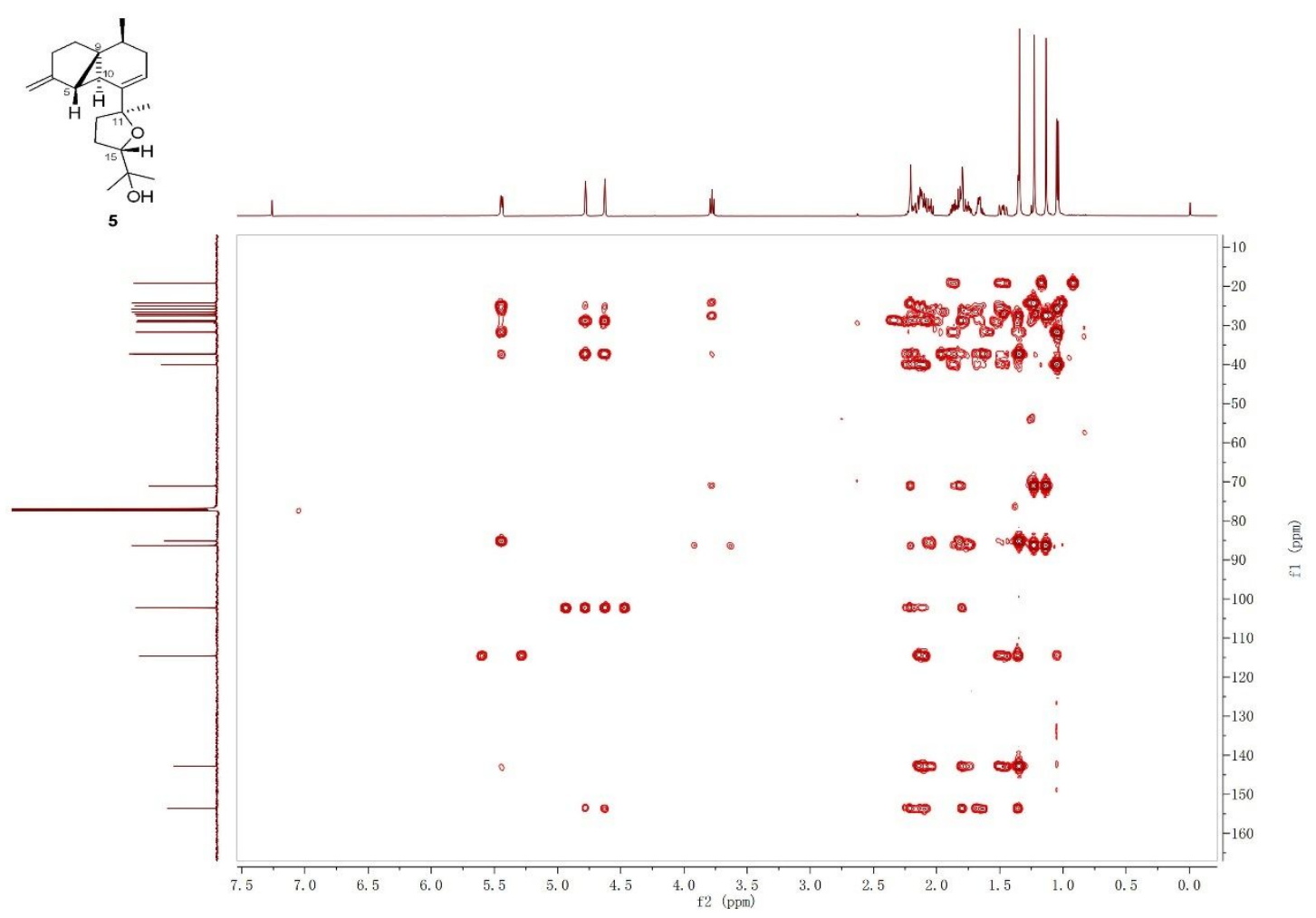


Figure S37. ${ }^{1} \mathrm{H}-{ }^{1} \mathrm{H}$ COSY spectrum of $5\left(500 \mathrm{MHz}, \mathrm{CDCl}_{3}\right)$

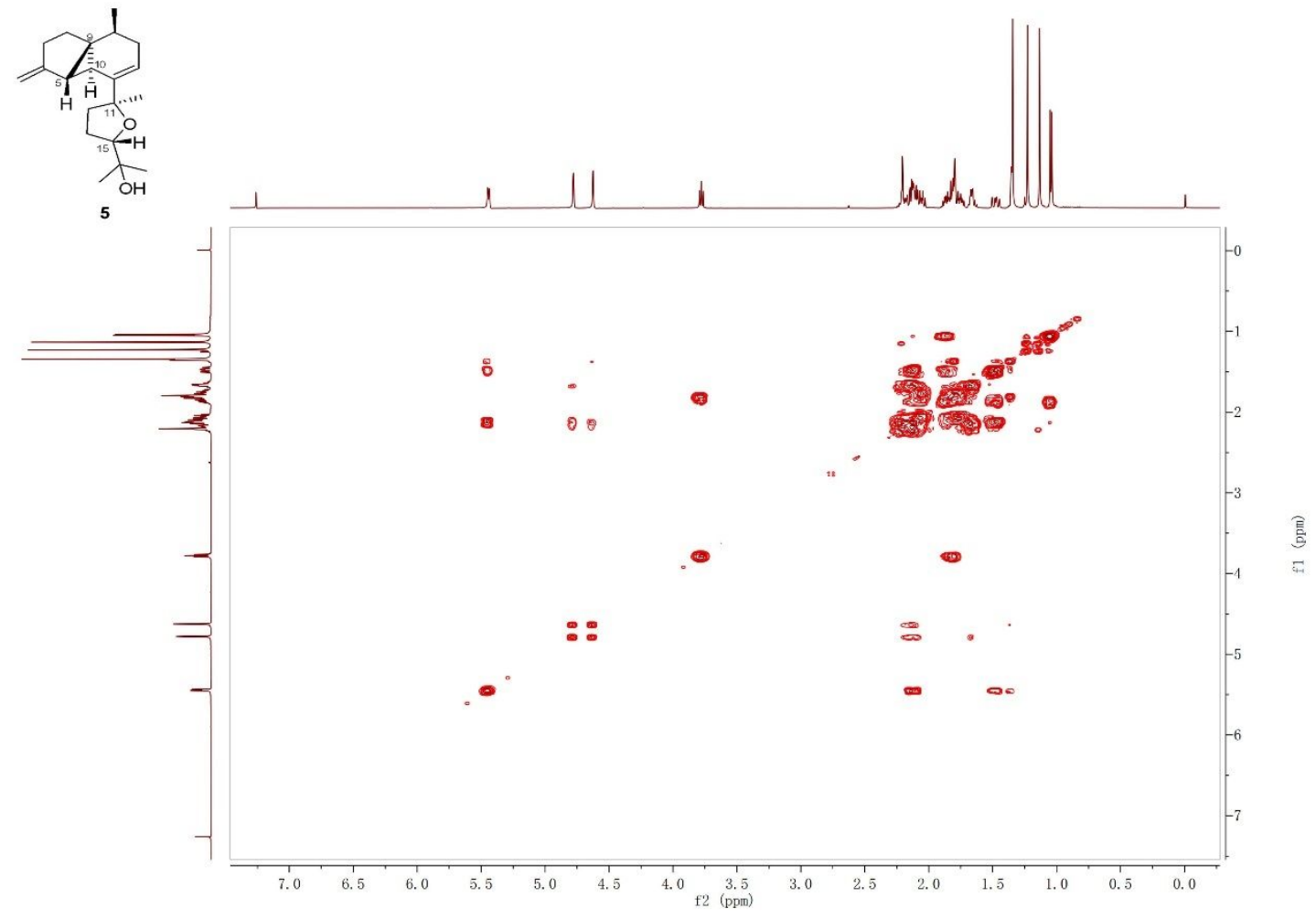

Figure S38. NOESY spectrum of $5\left(500 \mathrm{MHz}, \mathrm{CDCl}_{3}\right)$

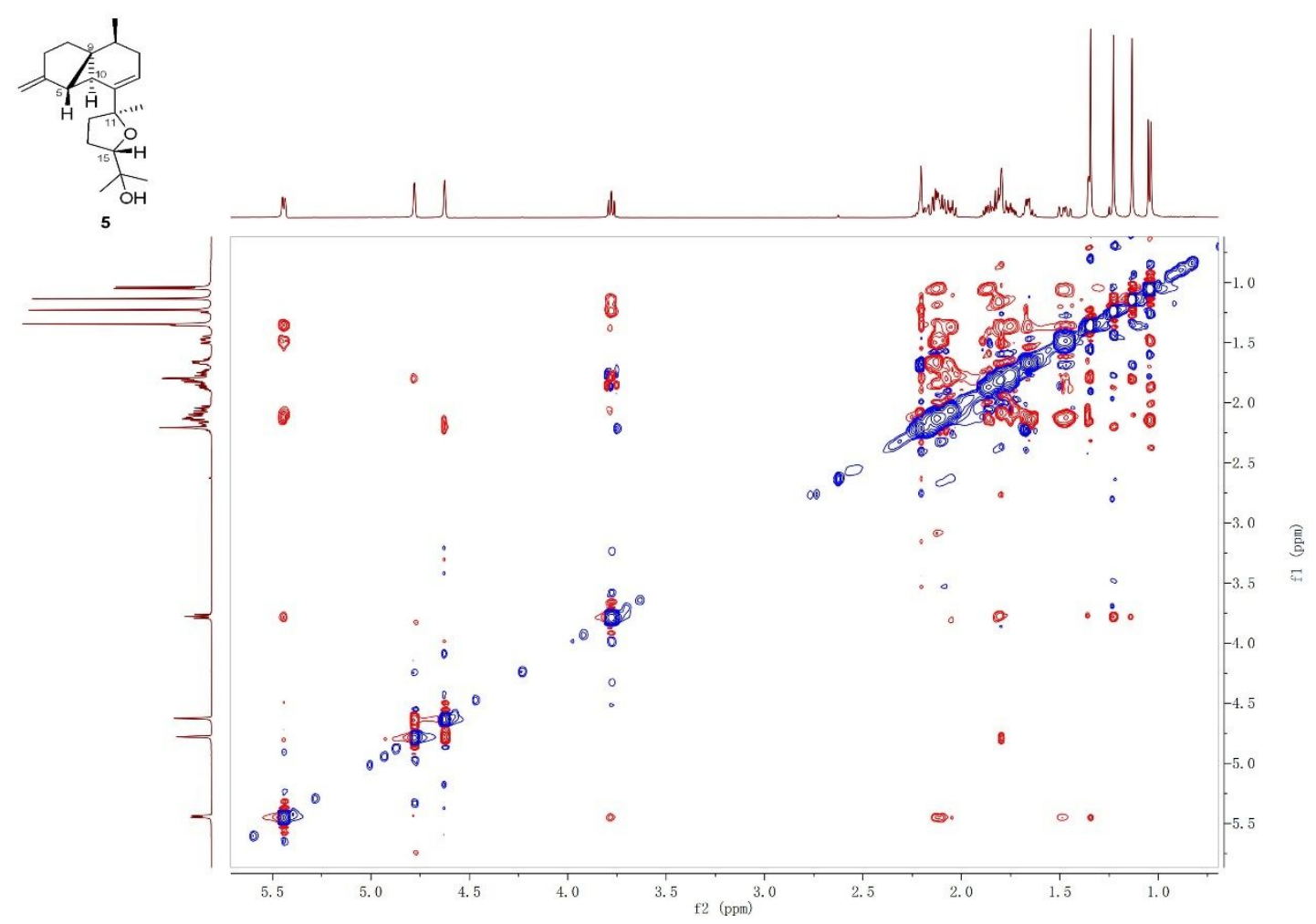


Figure S39. HR-ESI-MS spectrum of 5

Qualitative Analysis Report

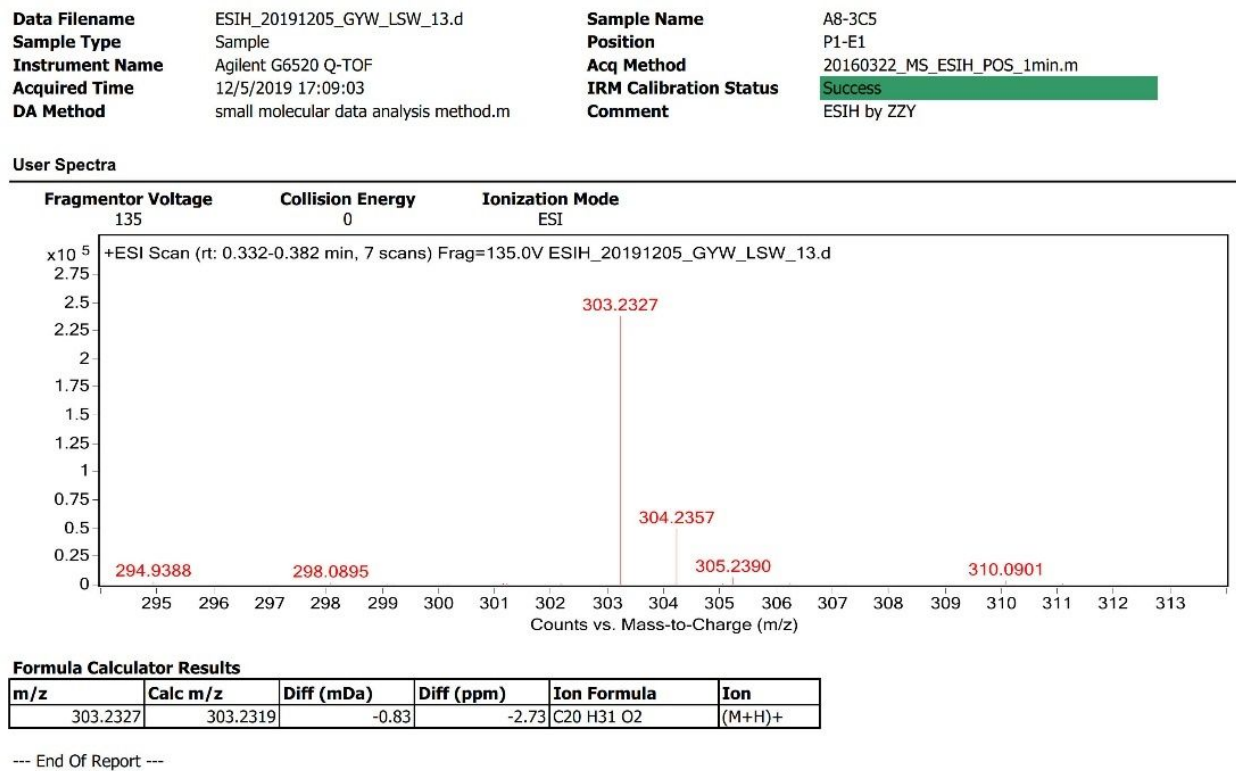

--- End Of Report --

A Agilent Technologies

Page 1 of 1

Printed at: $17: 23$ on: $12 / 5 / 2019$

Figure S40. IR spectrum of 5

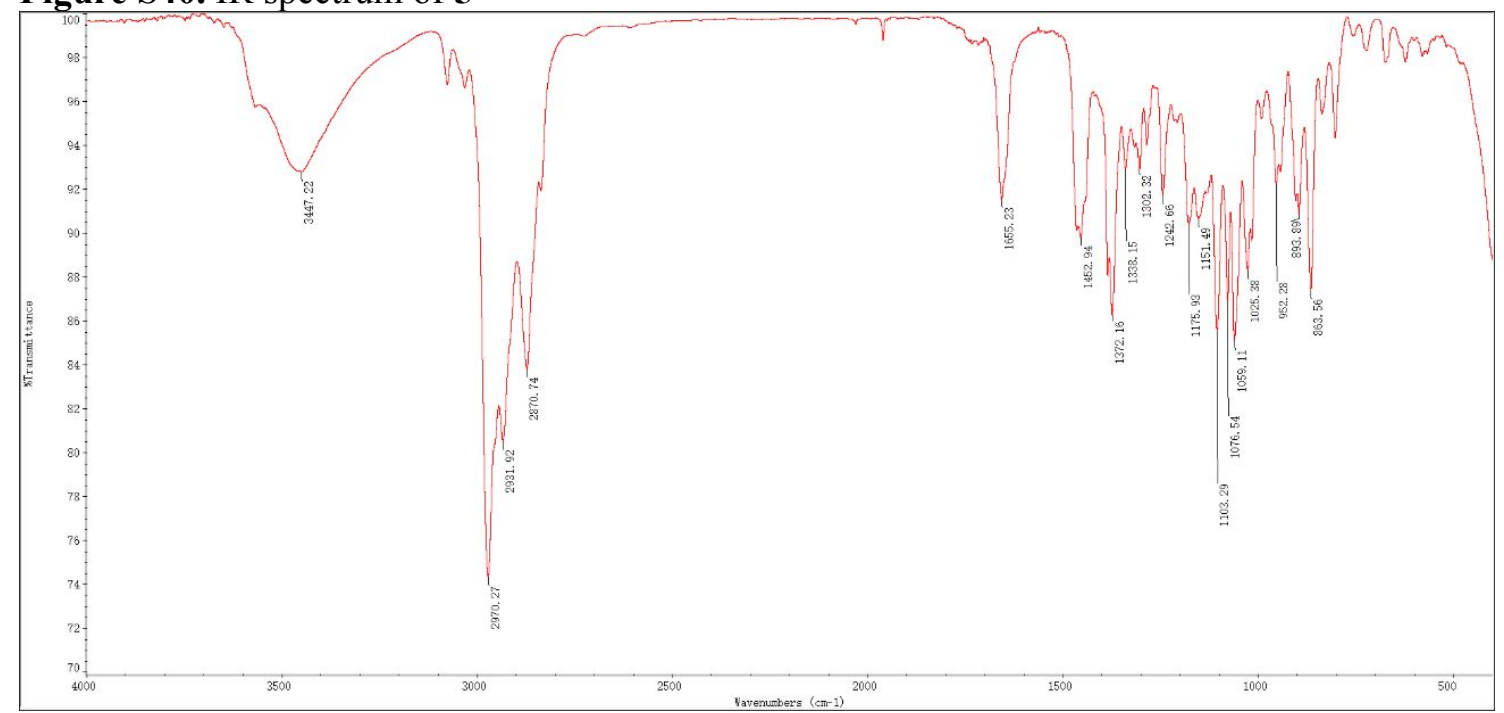


Figure S41. ${ }^{1} \mathrm{H}$ NMR spectrum of $\mathbf{5 a}\left(500 \mathrm{MHz}, \mathrm{CDCl}_{3}\right)$

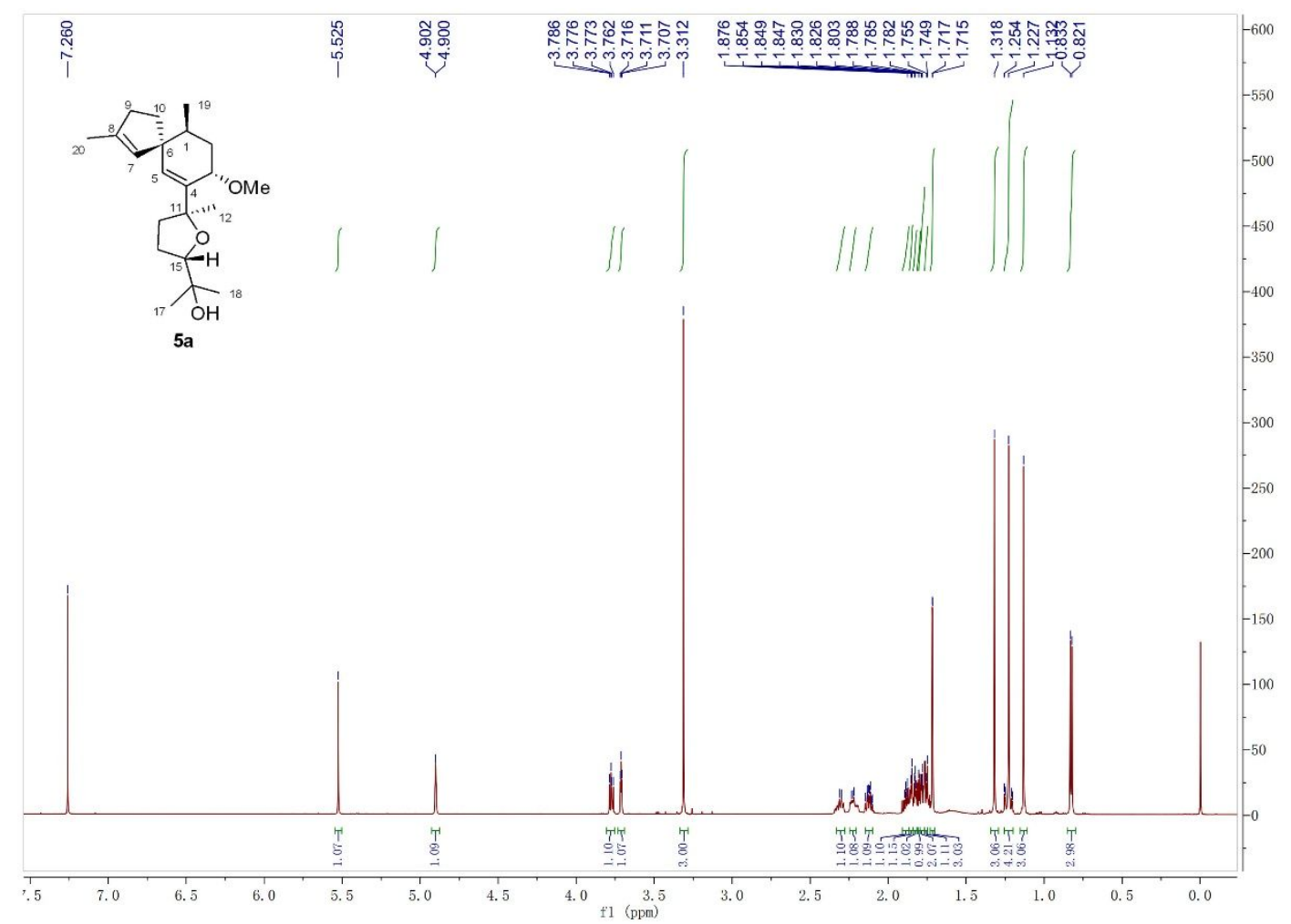

Figure $\mathbf{S 4 2 .}{ }^{13} \mathrm{C}\left\{{ }^{1} \mathrm{H}\right\}$ NMR spectrum of $\mathbf{5 a}\left(125 \mathrm{MHz}, \mathrm{CDCl}_{3}\right)$

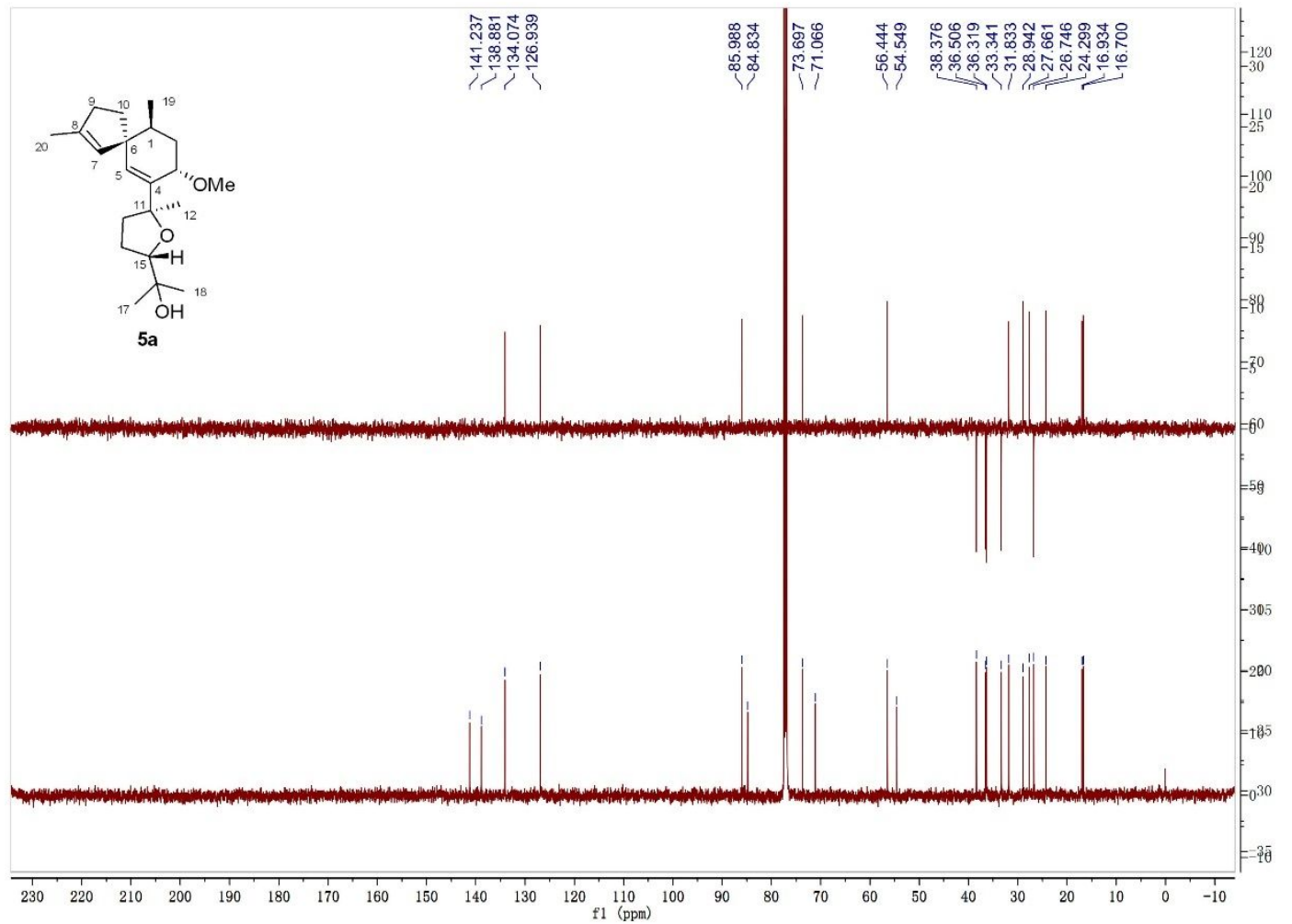


Figure S43. HSQC spectrum of $\mathbf{5 a}\left(500 \mathrm{MHz}, \mathrm{CDCl}_{3}\right)$

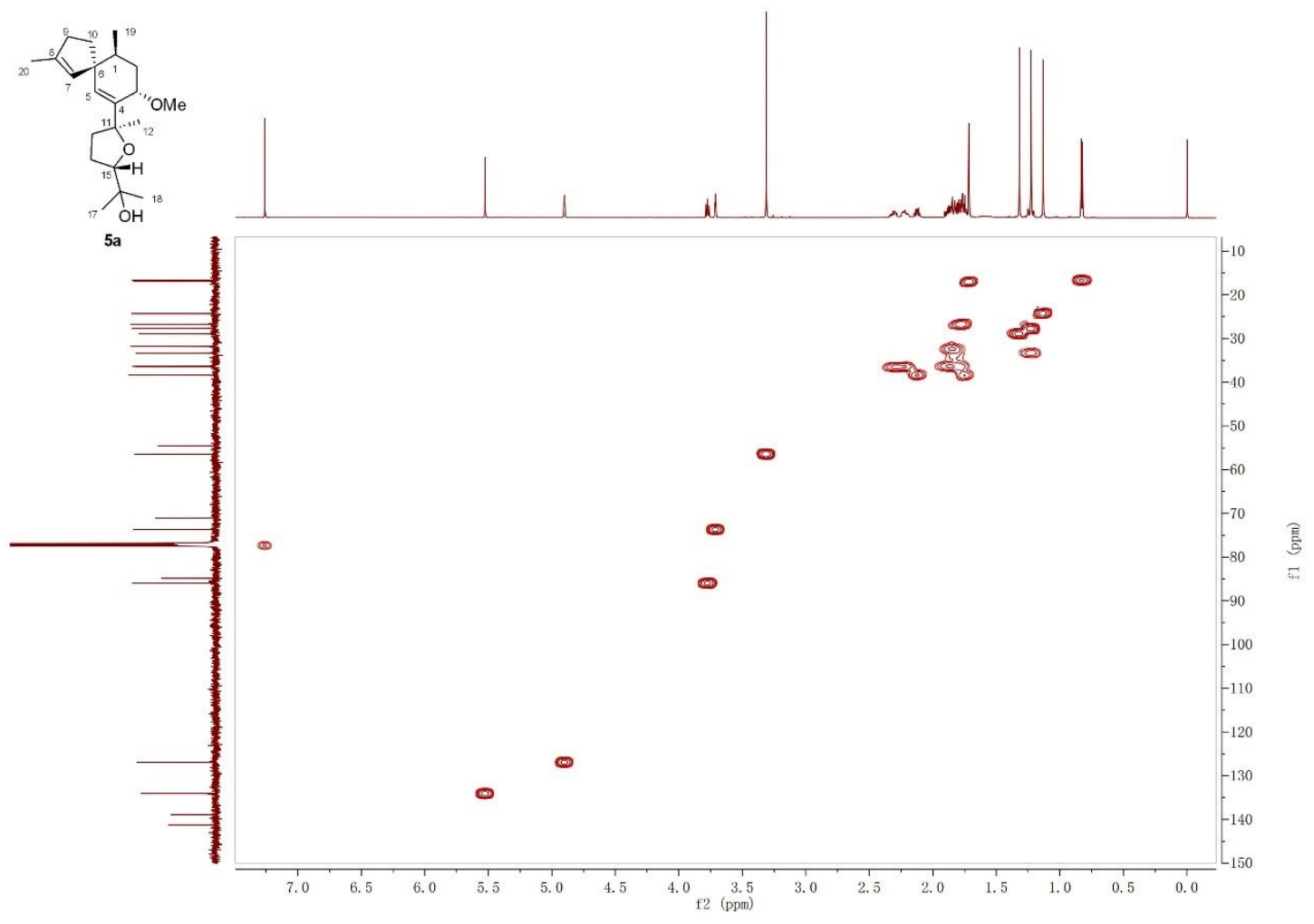

Figure S44. $\mathrm{HMBC}$ spectrum of $\mathbf{5 a}\left(500 \mathrm{MHz}, \mathrm{CDCl}_{3}\right)$

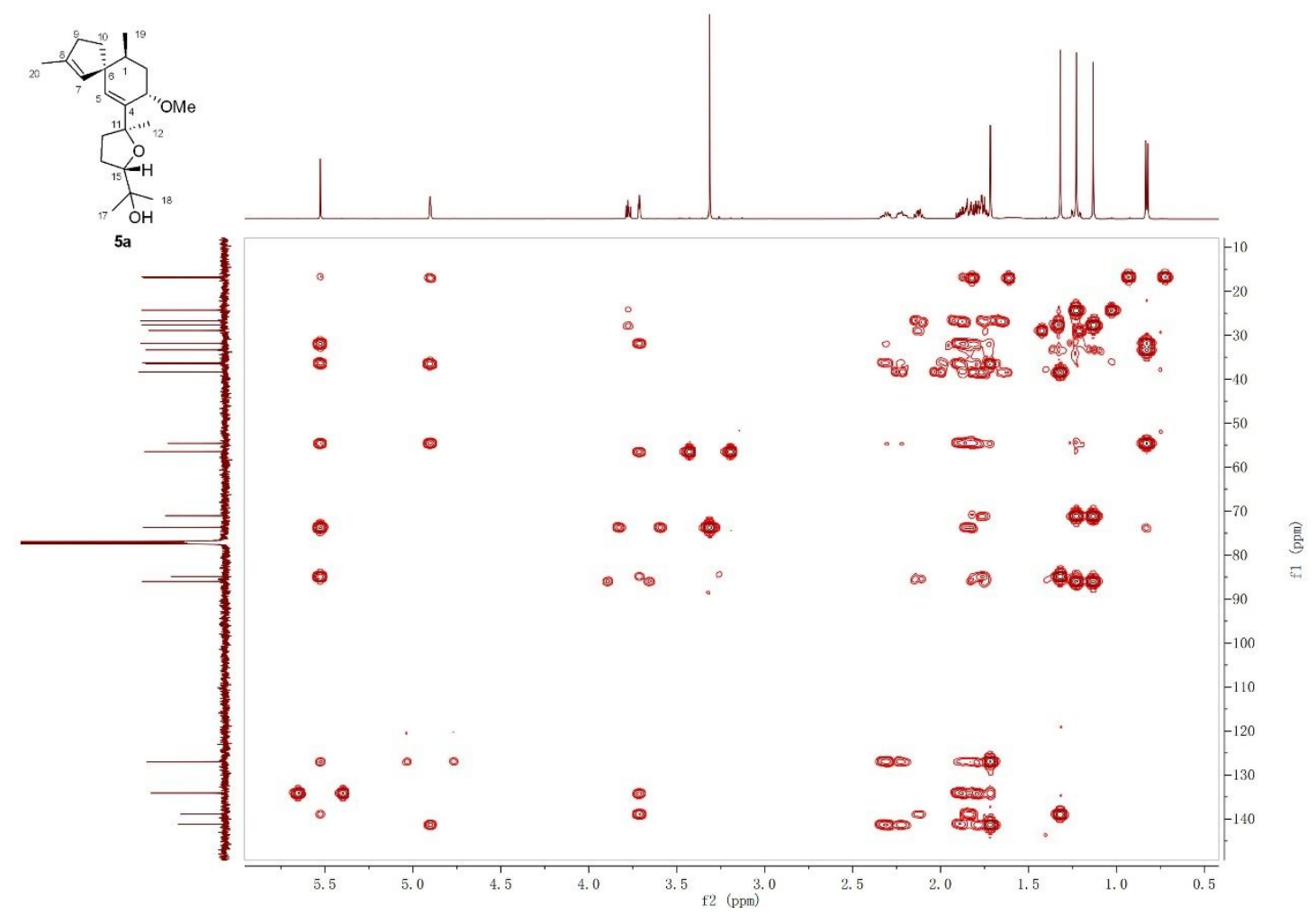


Figure S45. ${ }^{1} \mathrm{H}-{ }^{1} \mathrm{H}$ COSY spectrum of $5 \mathbf{a}\left(500 \mathrm{MHz}, \mathrm{CDCl}_{3}\right)$

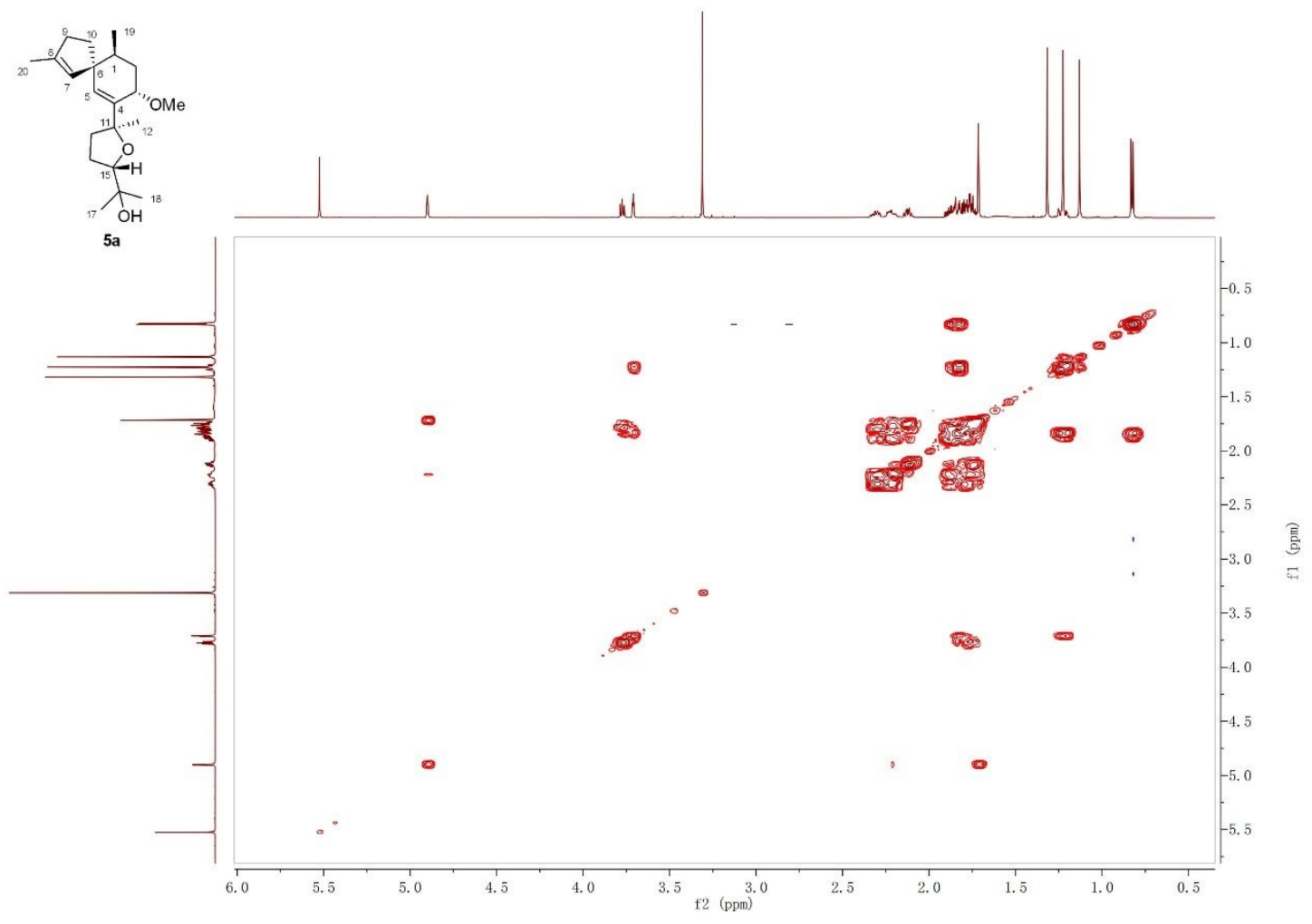

Figure S46. NOESY spectrum of $\mathbf{5 a}\left(500 \mathrm{MHz}, \mathrm{CDCl}_{3}\right)$

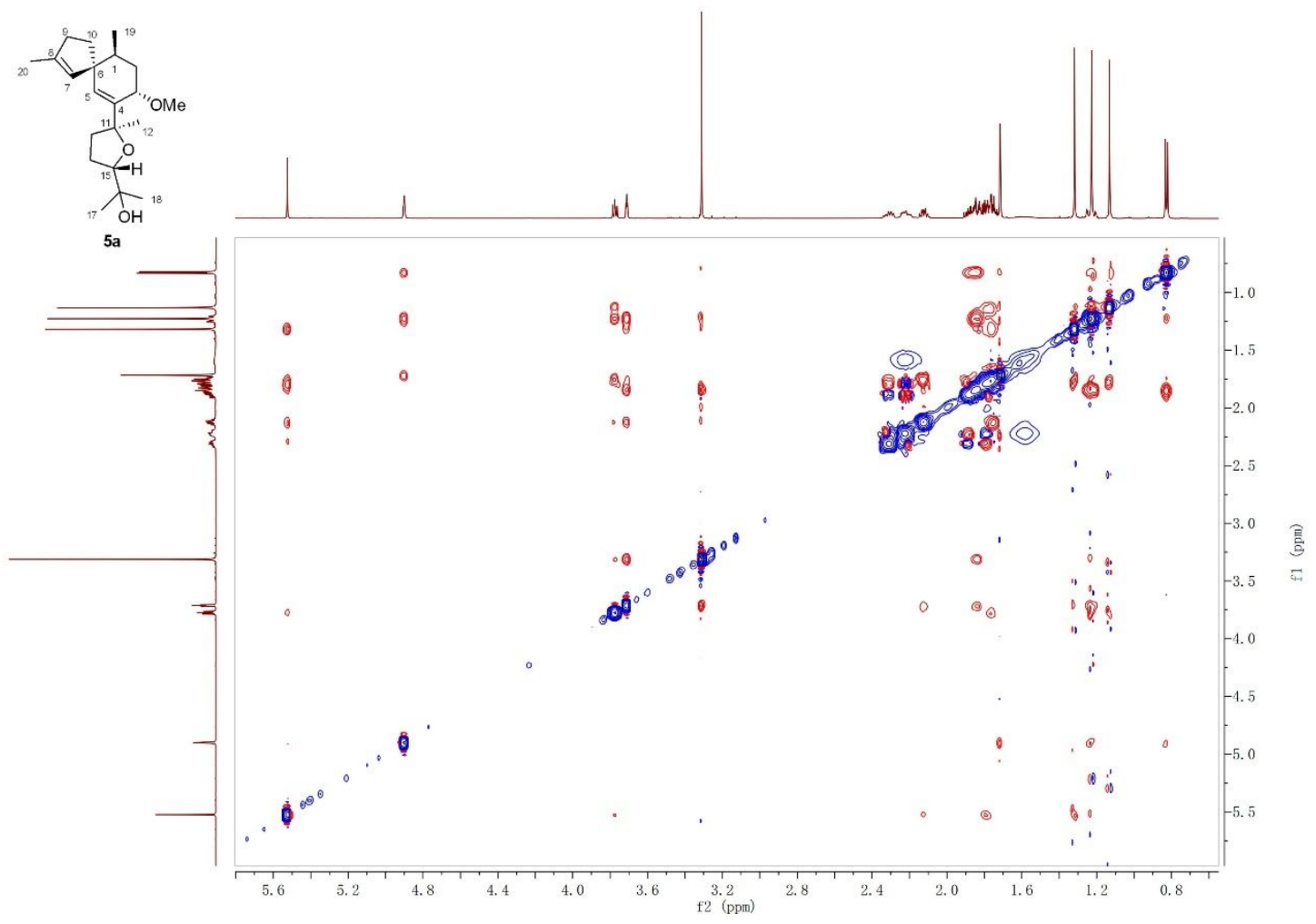


Figure S47. HR-ESI-MS spectrum of 5a

Qualitative Analysis Report

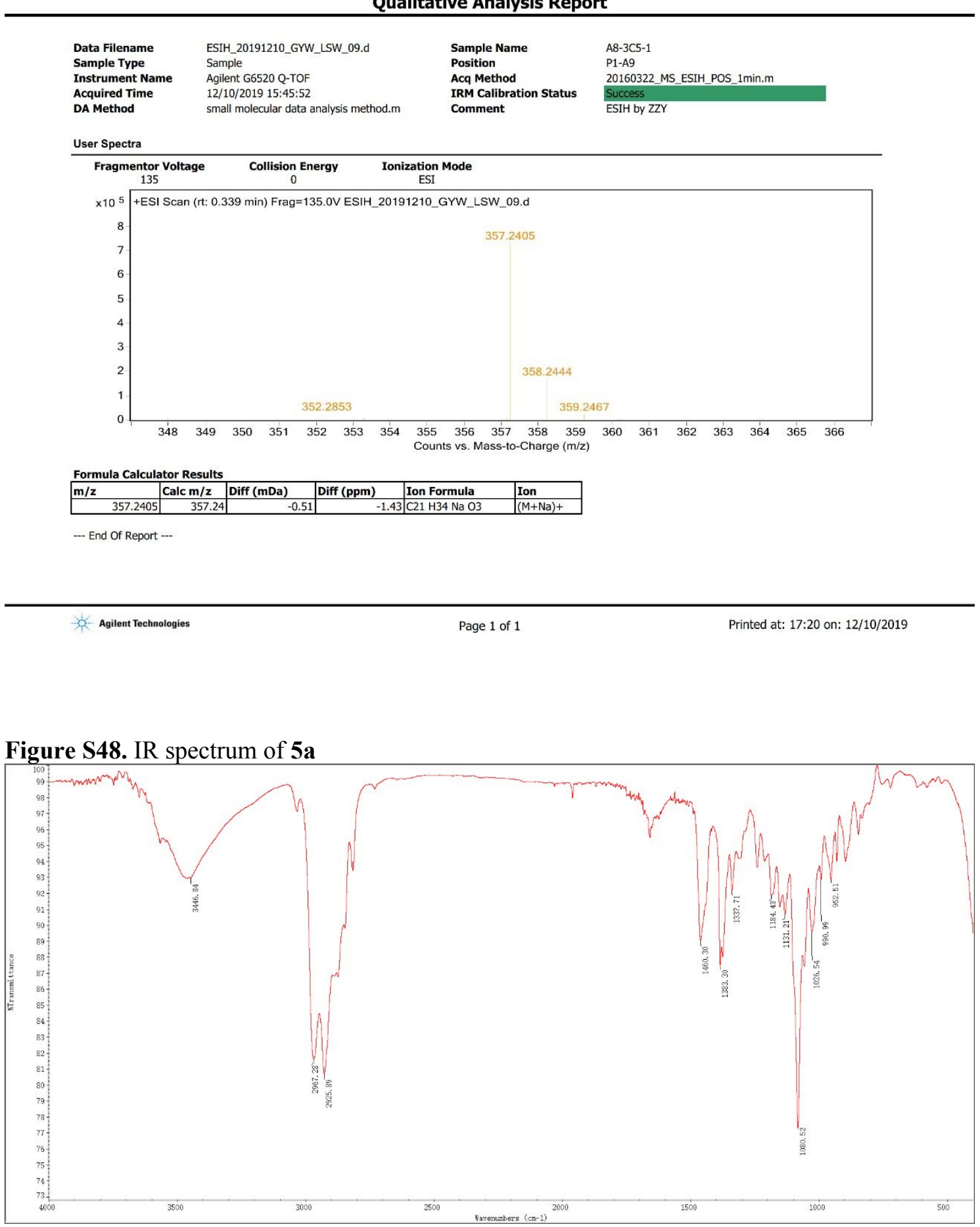


Figure S49. ${ }^{1} \mathrm{H}$ NMR spectrum of $\mathbf{5 b}\left(500 \mathrm{MHz}, \mathrm{CDCl}_{3}\right)$

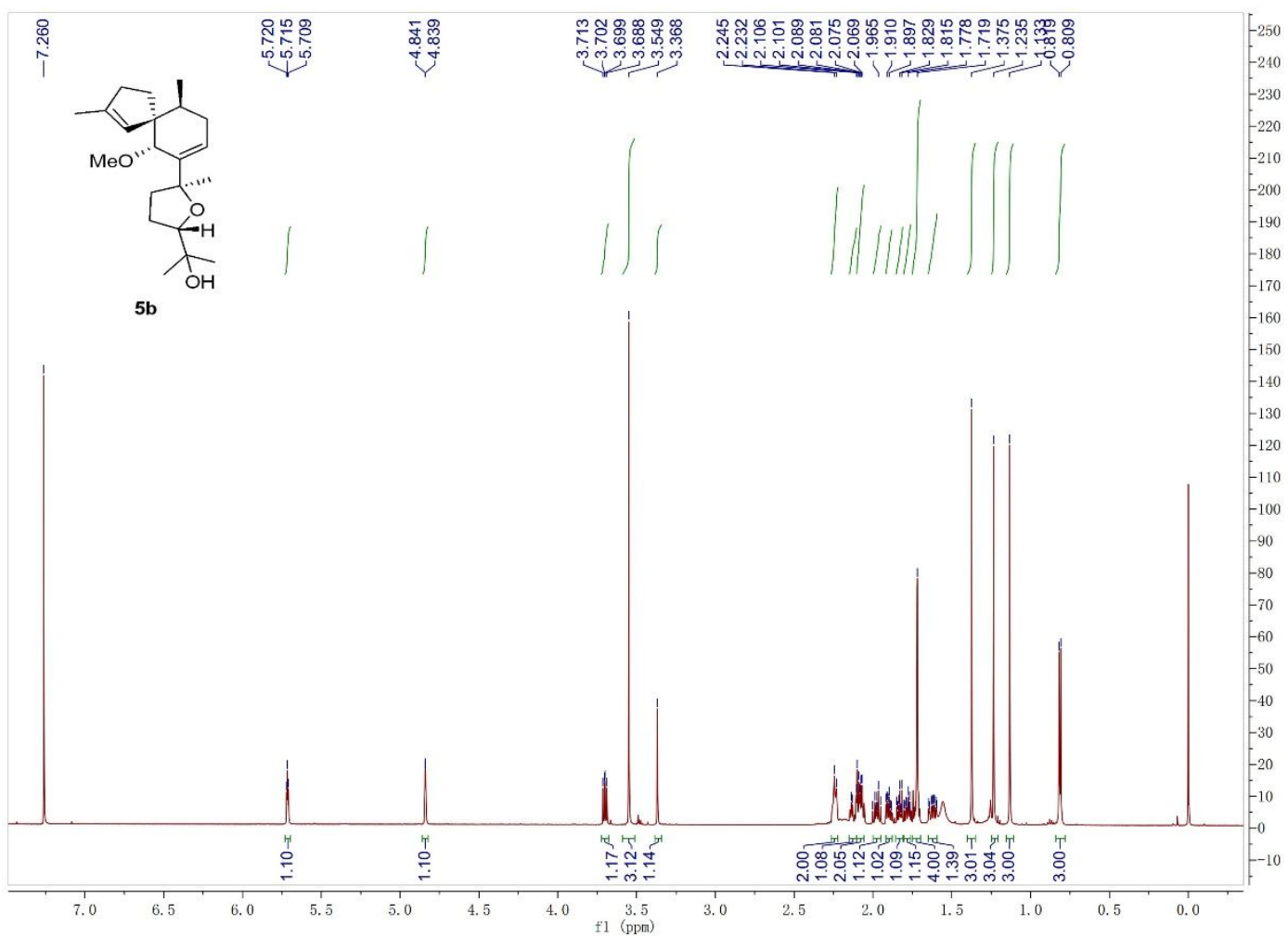

Figure S50. ${ }^{13} \mathrm{C}\left\{{ }^{1} \mathrm{H}\right\}$ NMR spectrum of $\mathbf{5 b}\left(125 \mathrm{MHz}, \mathrm{CDCl}_{3}\right)$

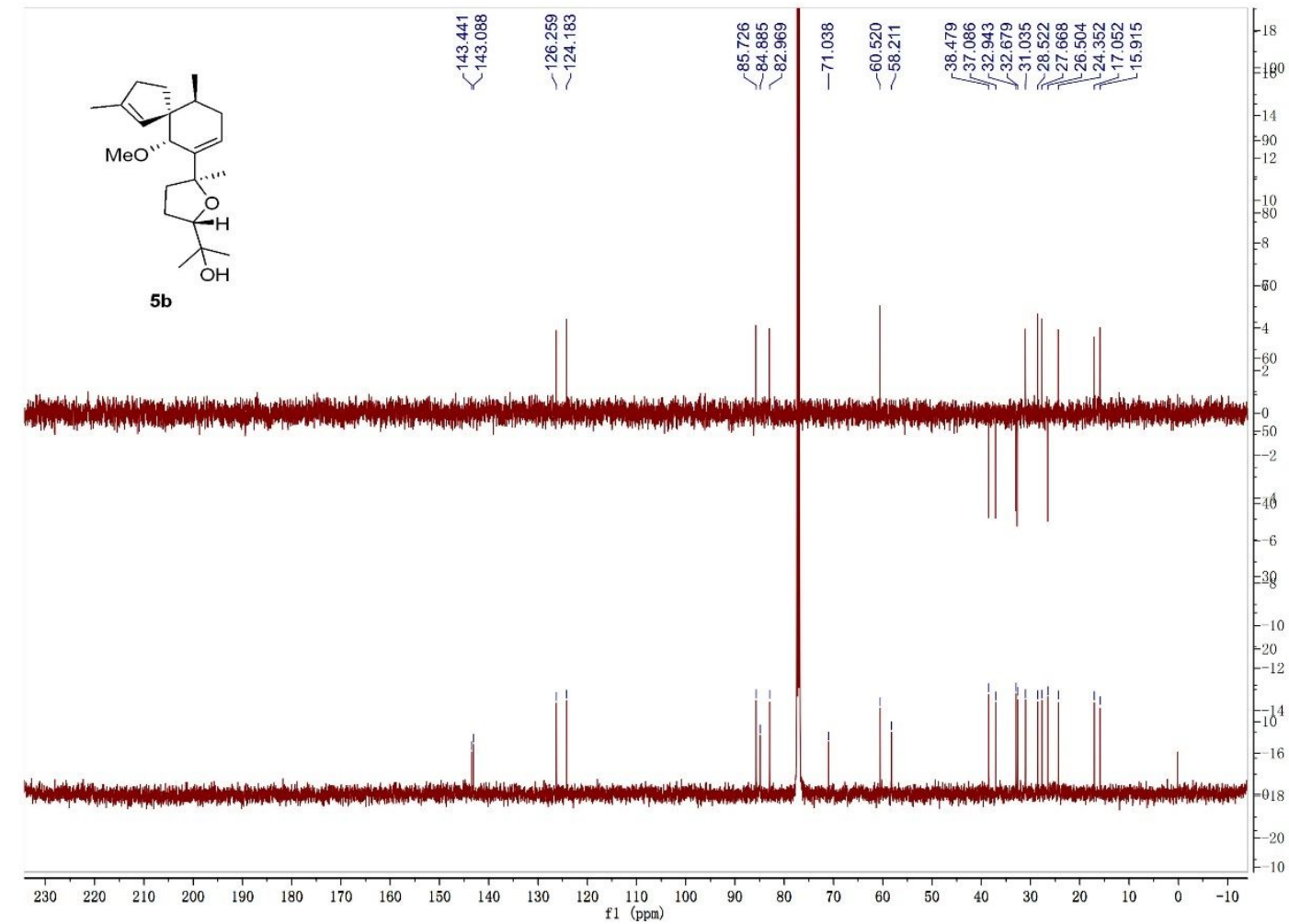


Figure S51. HSQC spectrum of $\mathbf{5 b}\left(500 \mathrm{MHz}, \mathrm{CDCl}_{3}\right)$

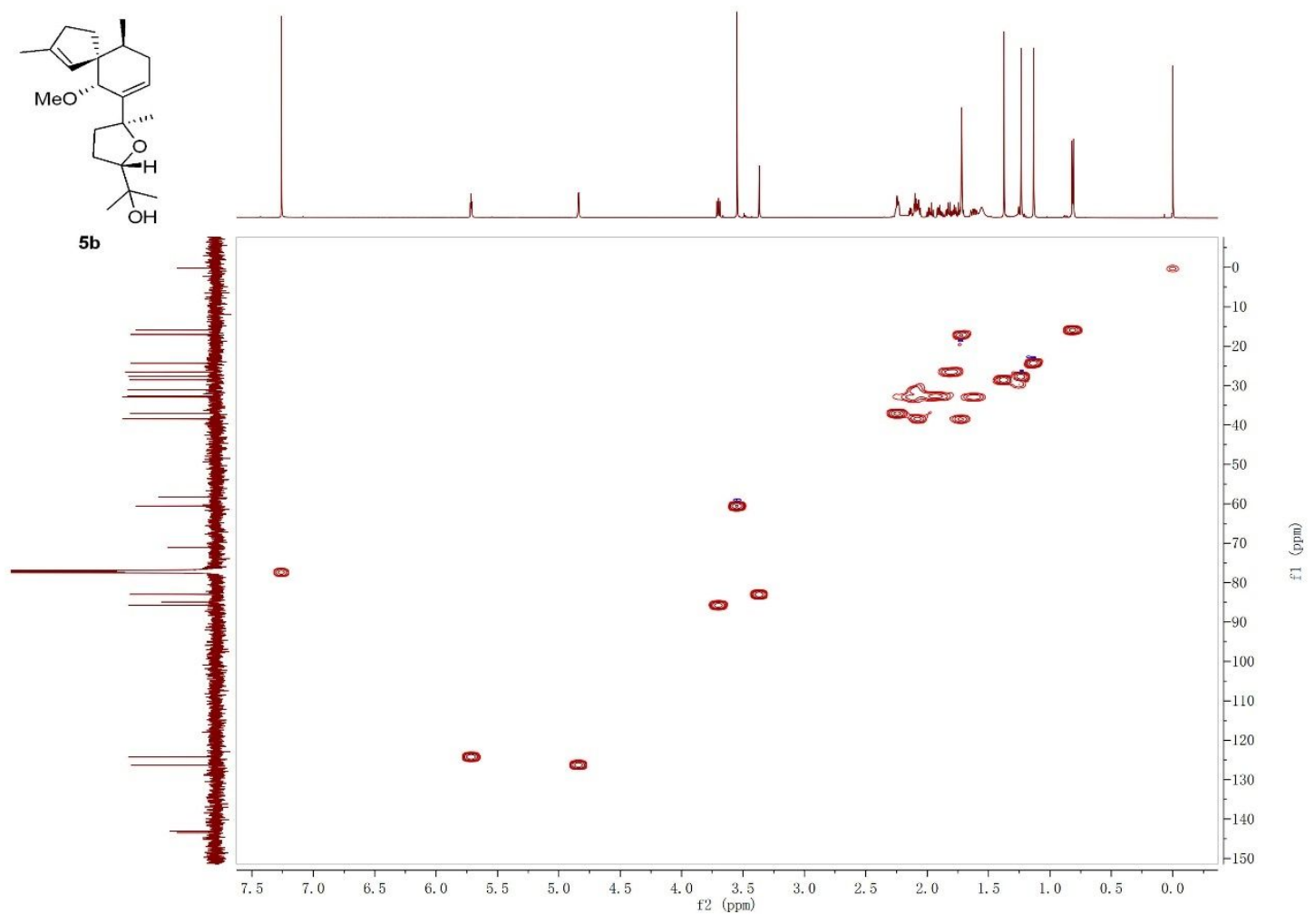

Figure S52. $\mathrm{HMBC}$ spectrum of $\mathbf{5 b}\left(500 \mathrm{MHz}, \mathrm{CDCl}_{3}\right)$

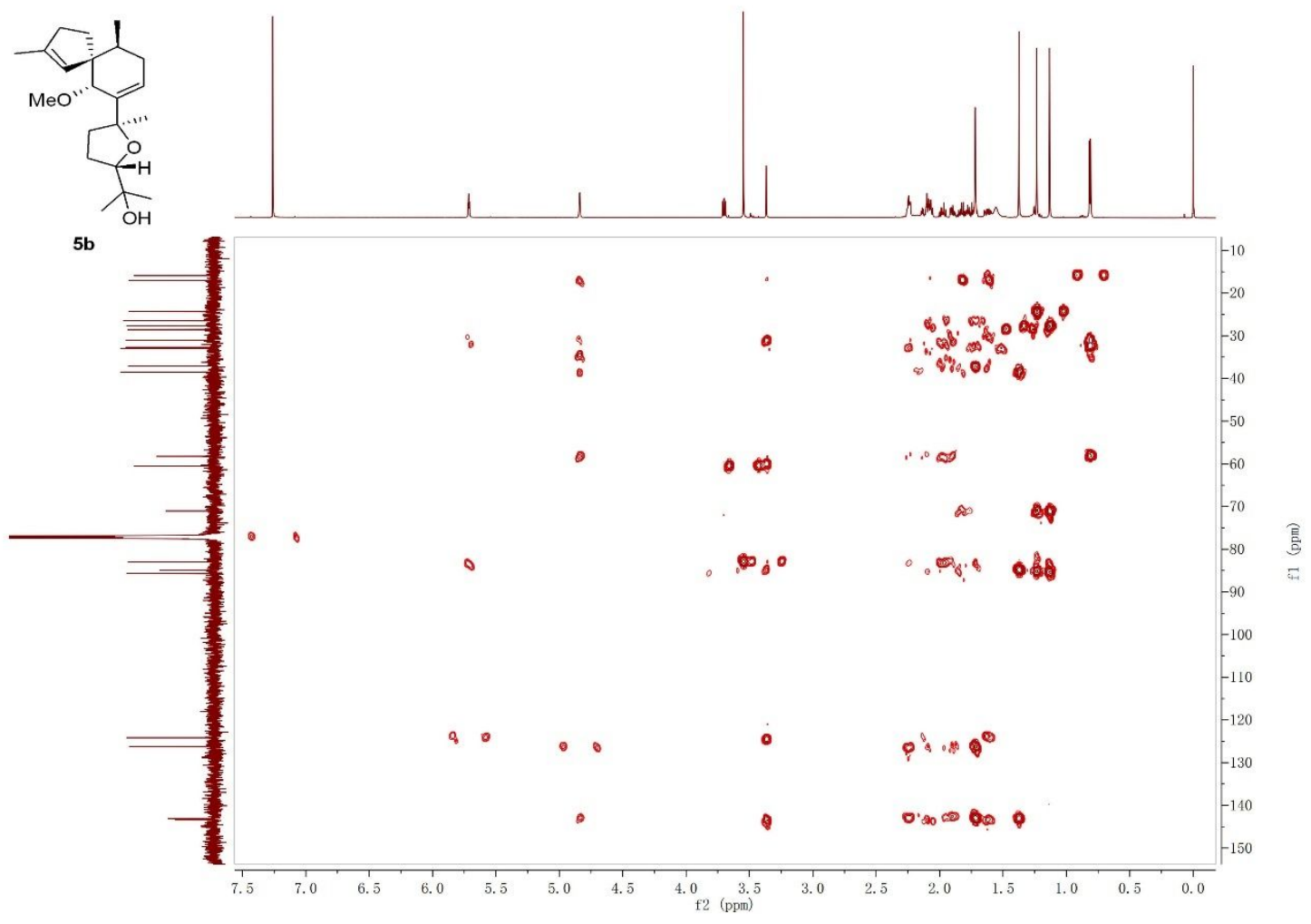

S31 
Figure S53. ${ }^{1} \mathrm{H}-{ }^{1} \mathrm{H}$ COSY spectrum of $\mathbf{5 b}\left(500 \mathrm{MHz}, \mathrm{CDCl}_{3}\right)$

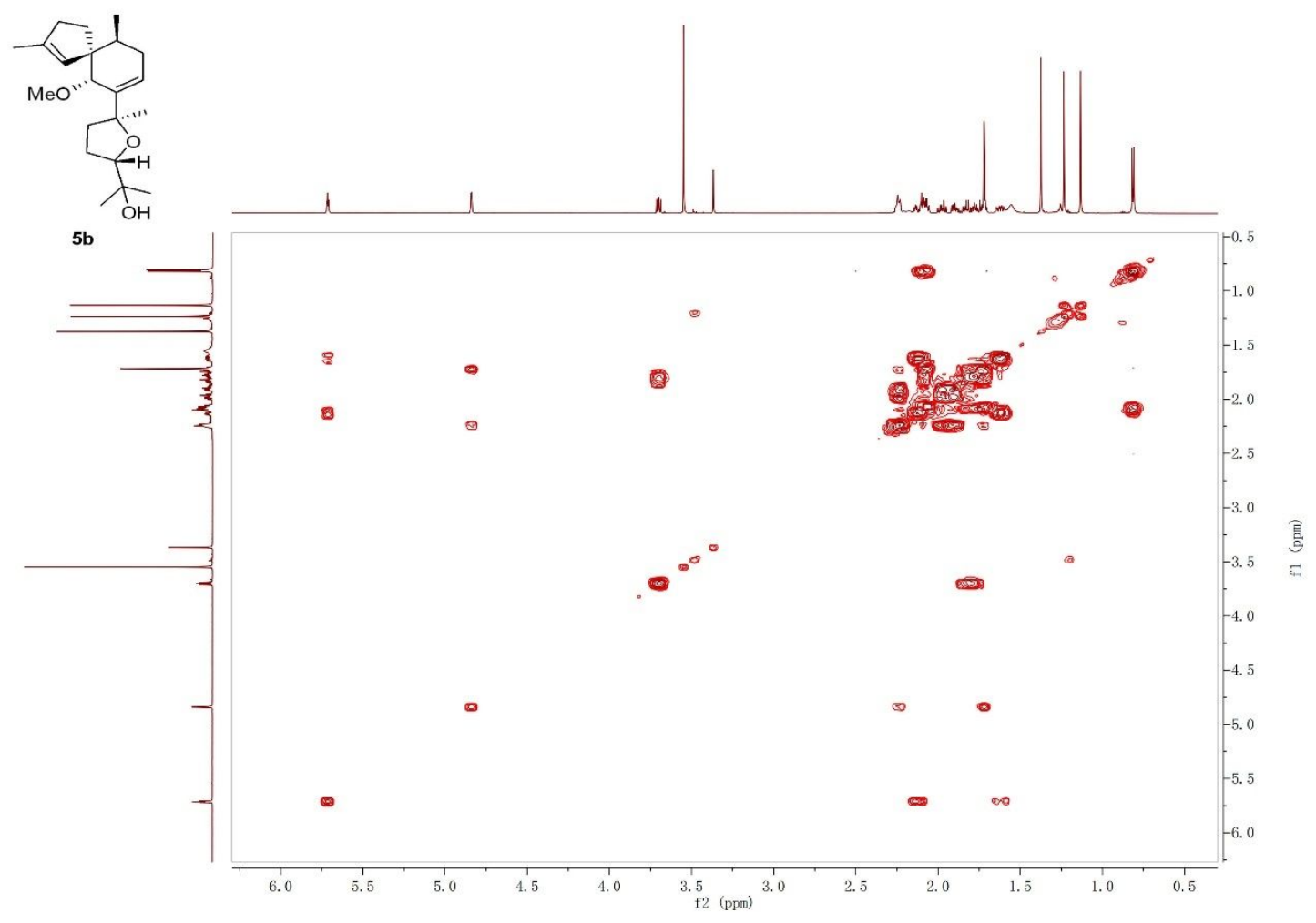

Figure S54. NOESY spectrum of $\mathbf{5 b}\left(500 \mathrm{MHz}, \mathrm{CDCl}_{3}\right)$
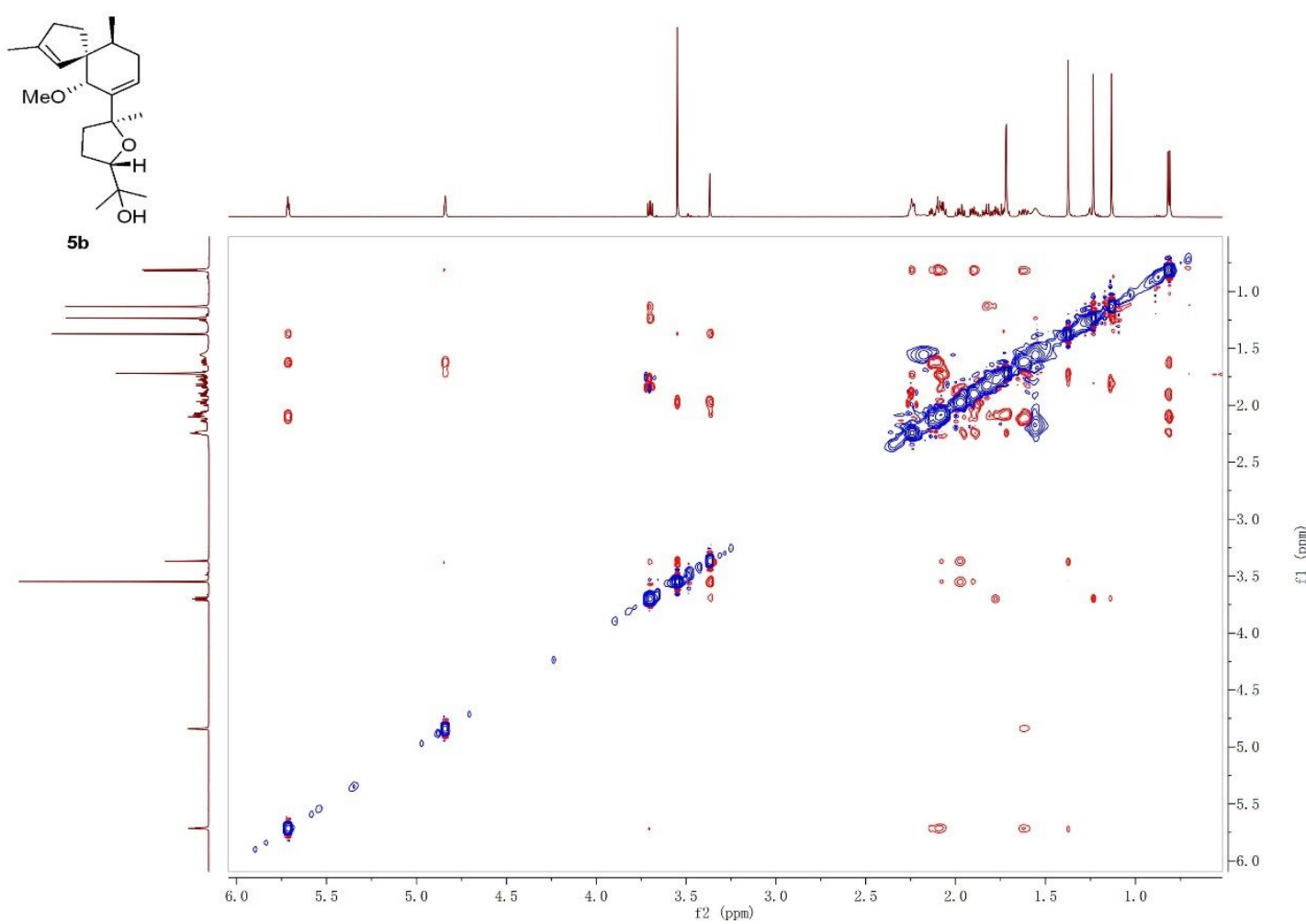
Figure S55. HR-ESI-MS spectrum of 5b

Qualitative Analysis Report

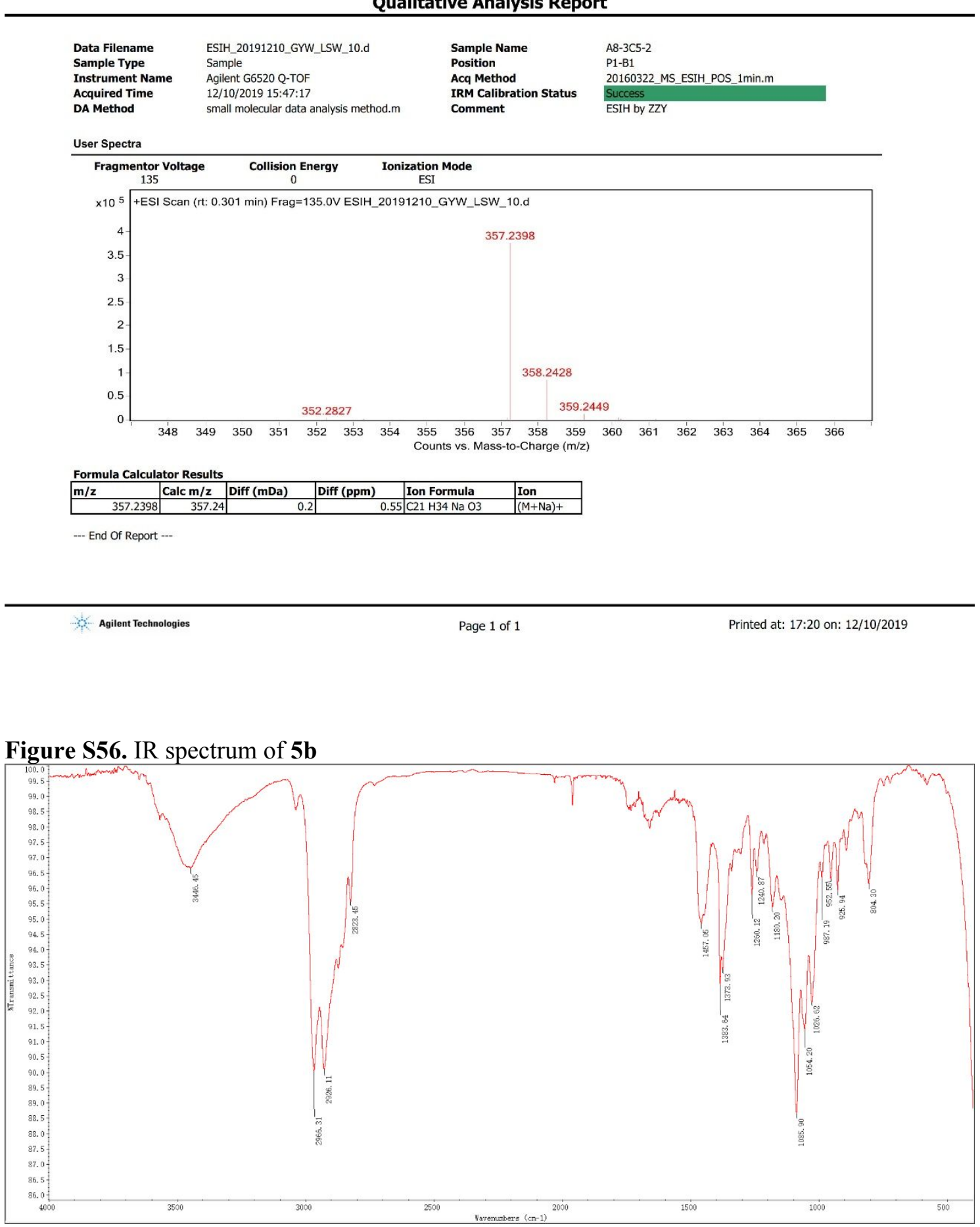


Figure S57. ${ }^{1} \mathrm{H}$ NMR spectrum of $6\left(500 \mathrm{MHz}, \mathrm{CDCl}_{3}\right)$

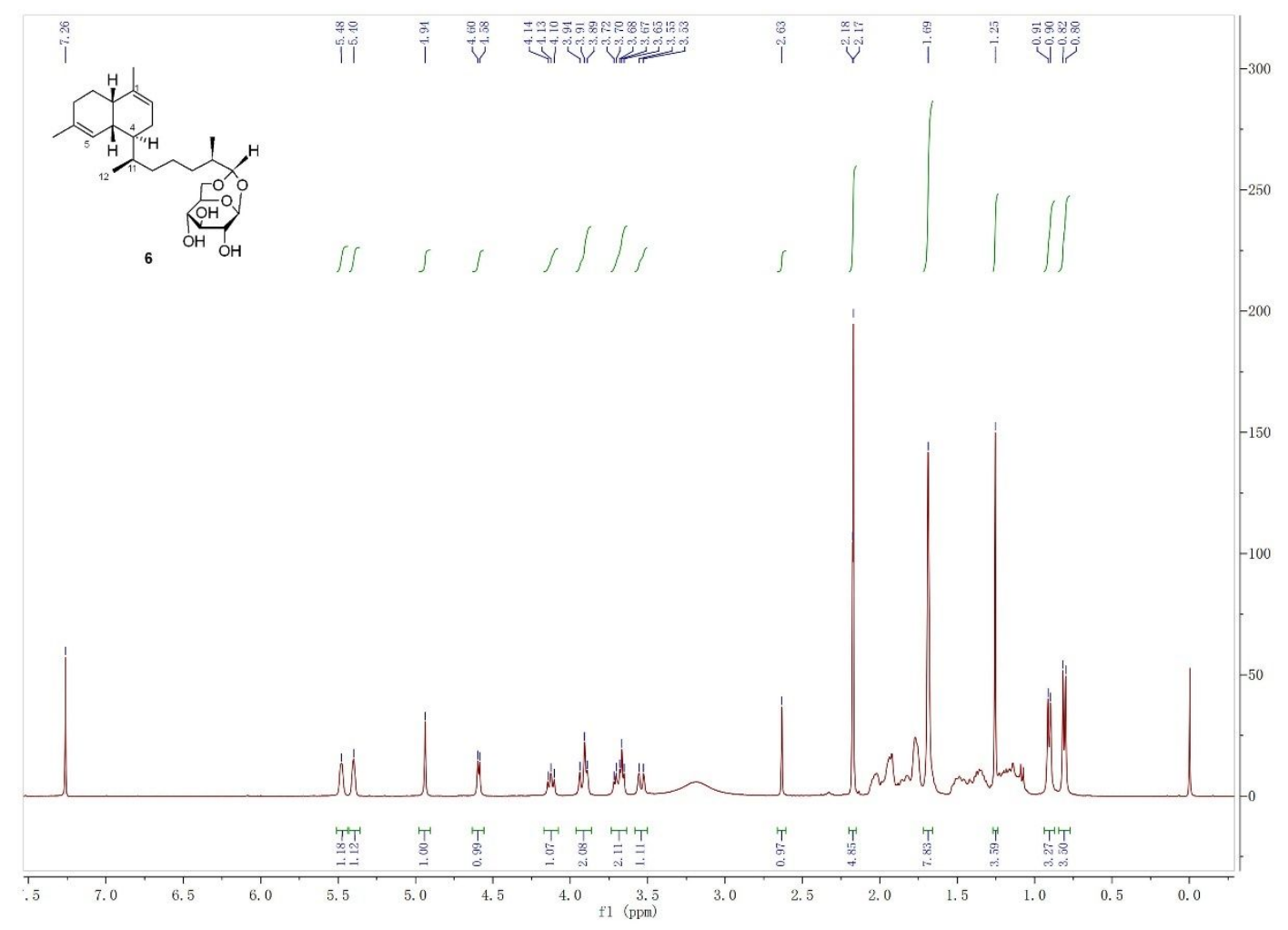

Figure S58. ${ }^{13} \mathrm{C}\left\{{ }^{1} \mathrm{H}\right\}$ NMR spectrum of $6\left(125 \mathrm{MHz}, \mathrm{CDCl}_{3}\right)$

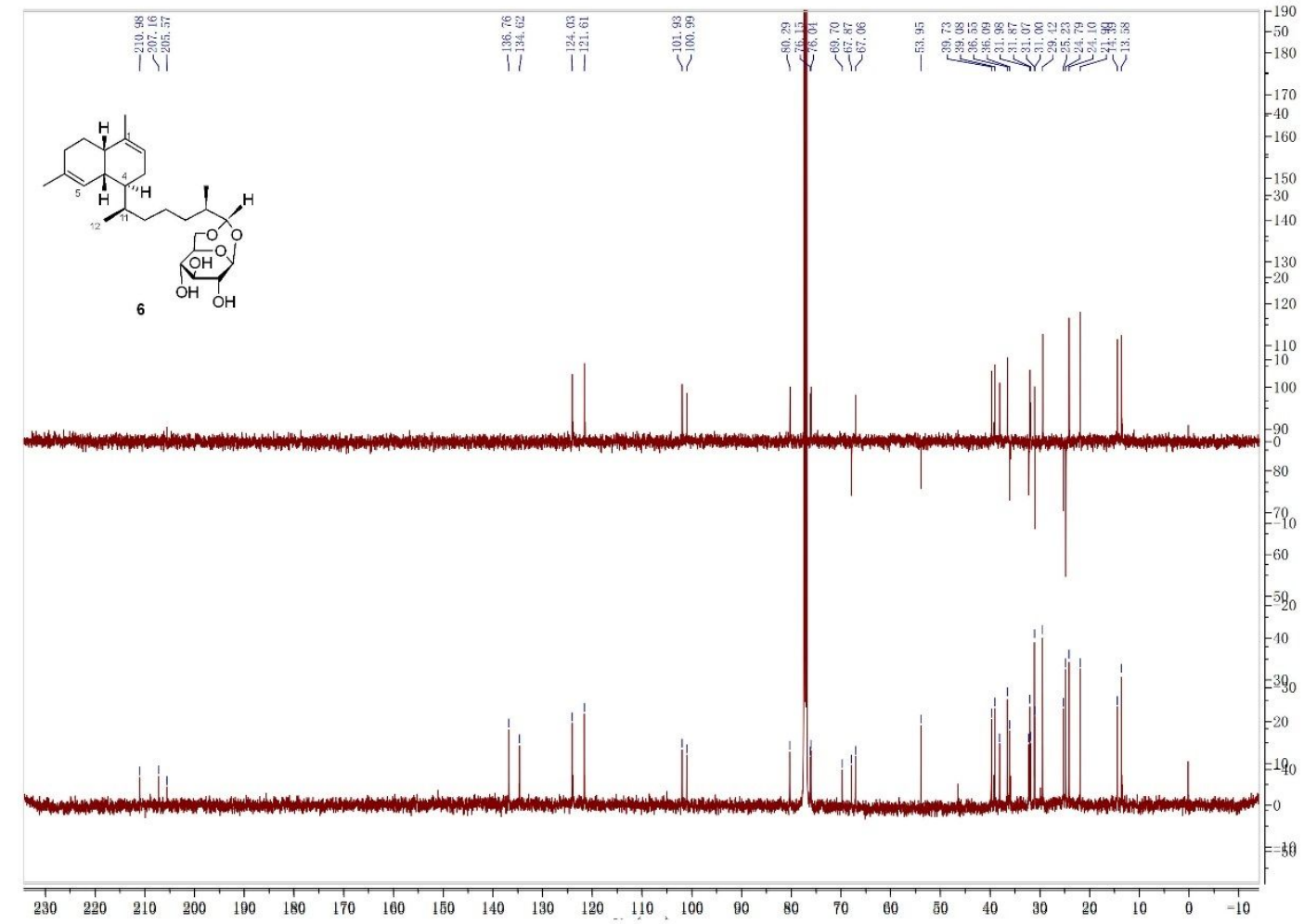


Figure S59. ${ }^{1} \mathrm{H}$ NMR spectrum of $6 \mathbf{6}\left(500 \mathrm{MHz}, \mathrm{CDCl}_{3}\right)$

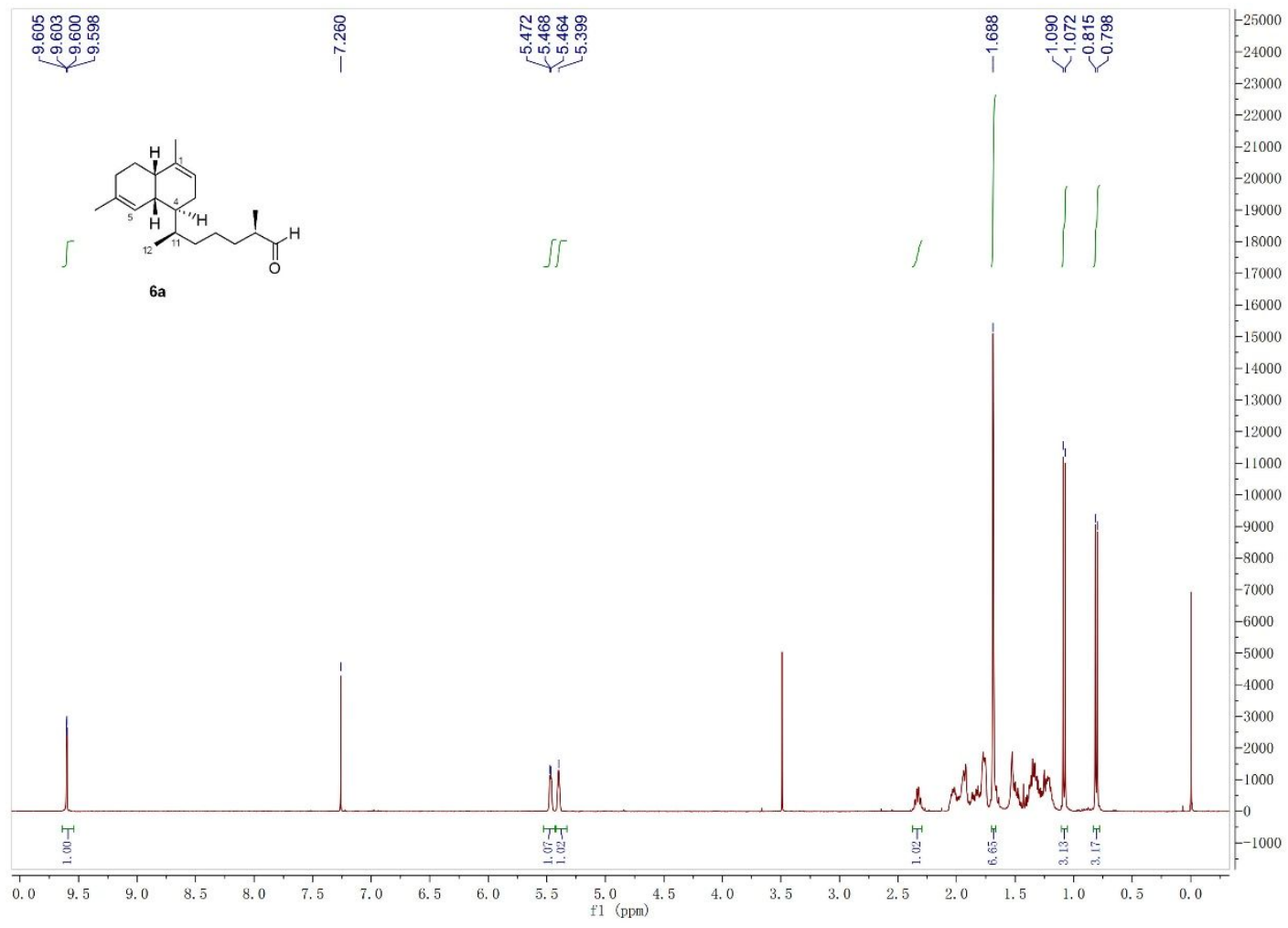

Figure S60. ${ }^{13} \mathrm{C}\left\{{ }^{1} \mathrm{H}\right\}$ NMR spectrum of $\mathbf{6 a}\left(125 \mathrm{MHz}, \mathrm{CDCl}_{3}\right)$

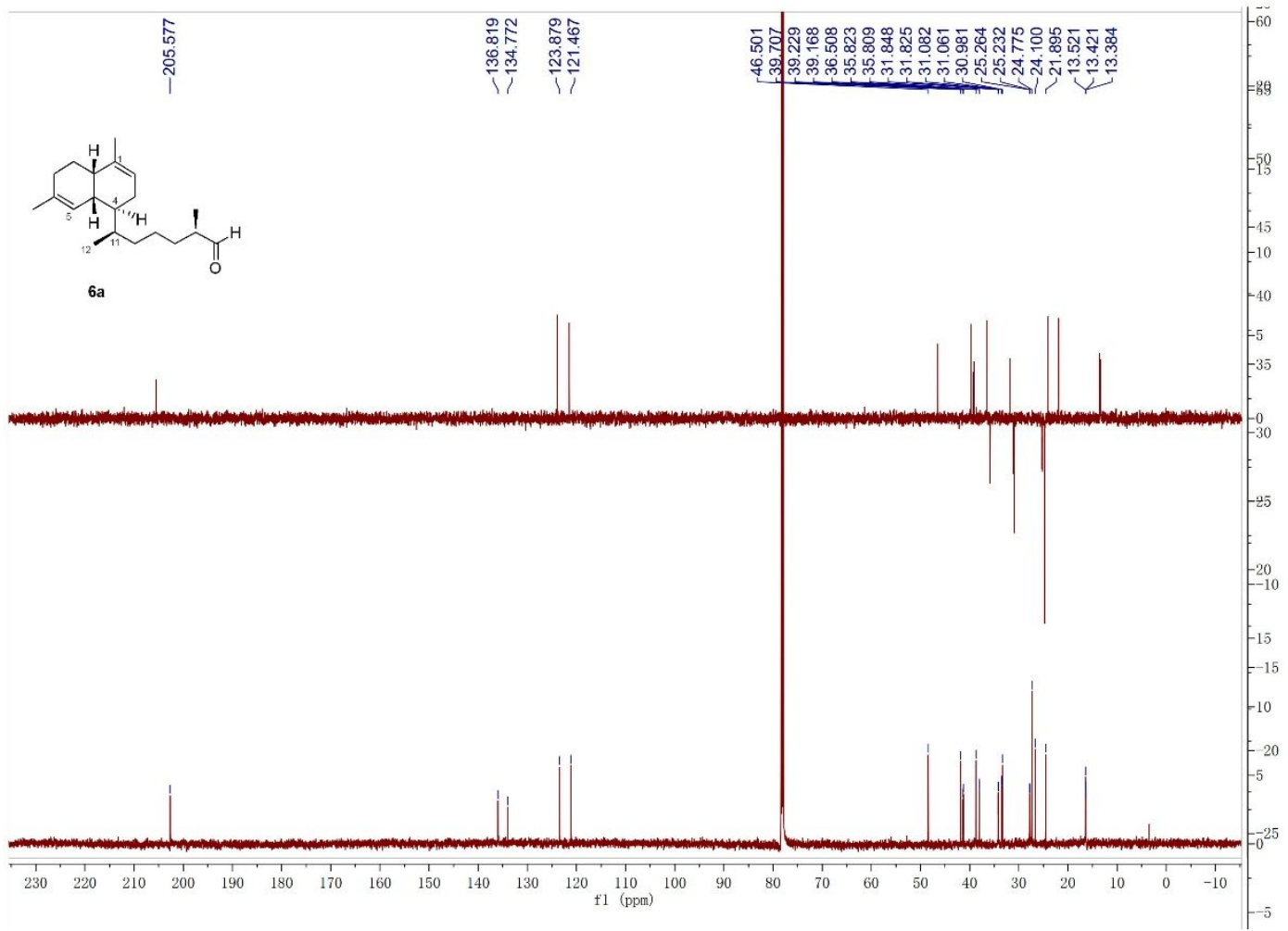


Figure S61. ${ }^{1} \mathrm{H}$ NMR spectrum of $7\left(400 \mathrm{MHz}, \mathrm{CDCl}_{3}\right)$

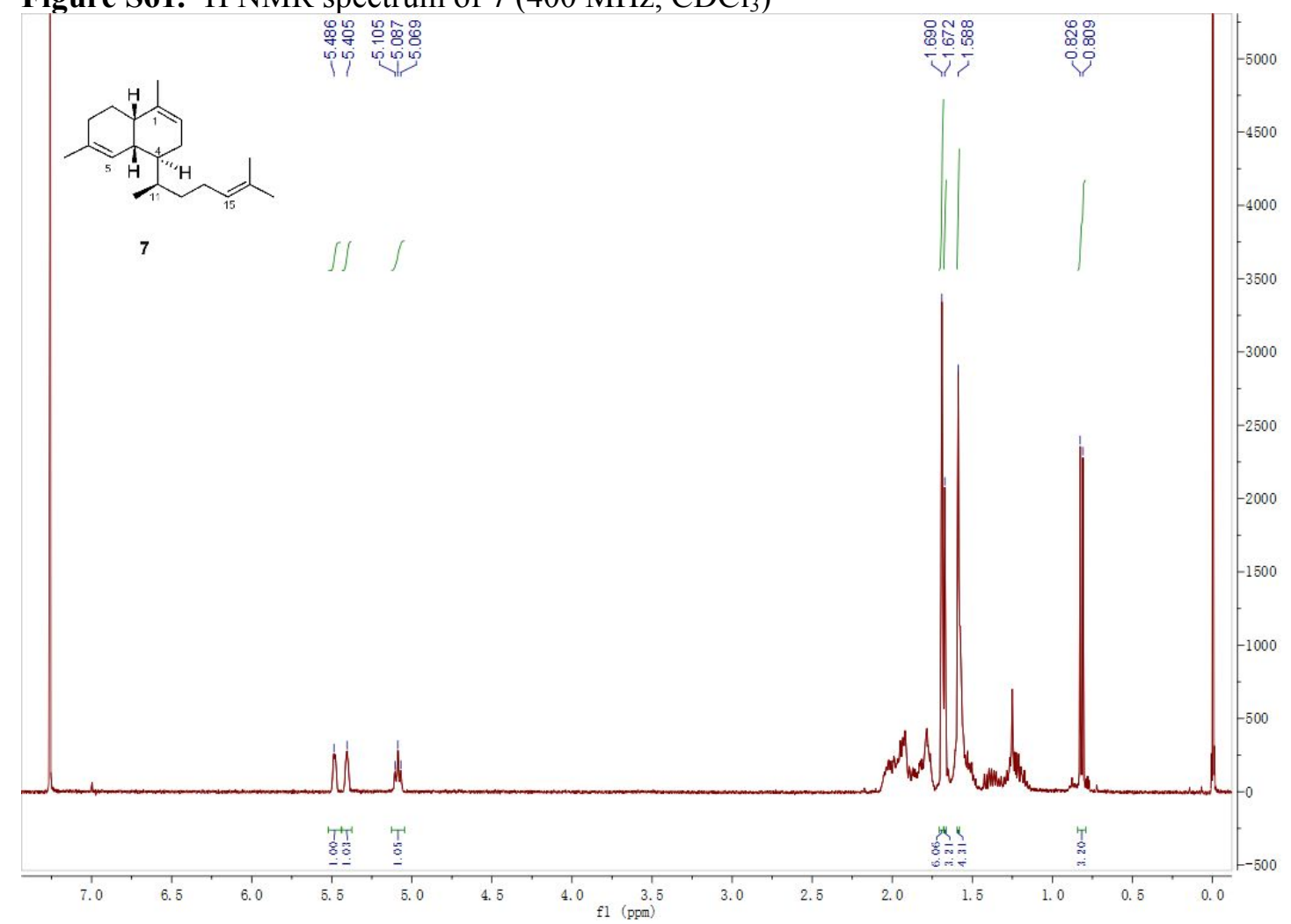

Figure S62. ${ }^{1} \mathrm{H}$ NMR spectrum of $8\left(500 \mathrm{MHz}, \mathrm{CDCl}_{3}\right)$

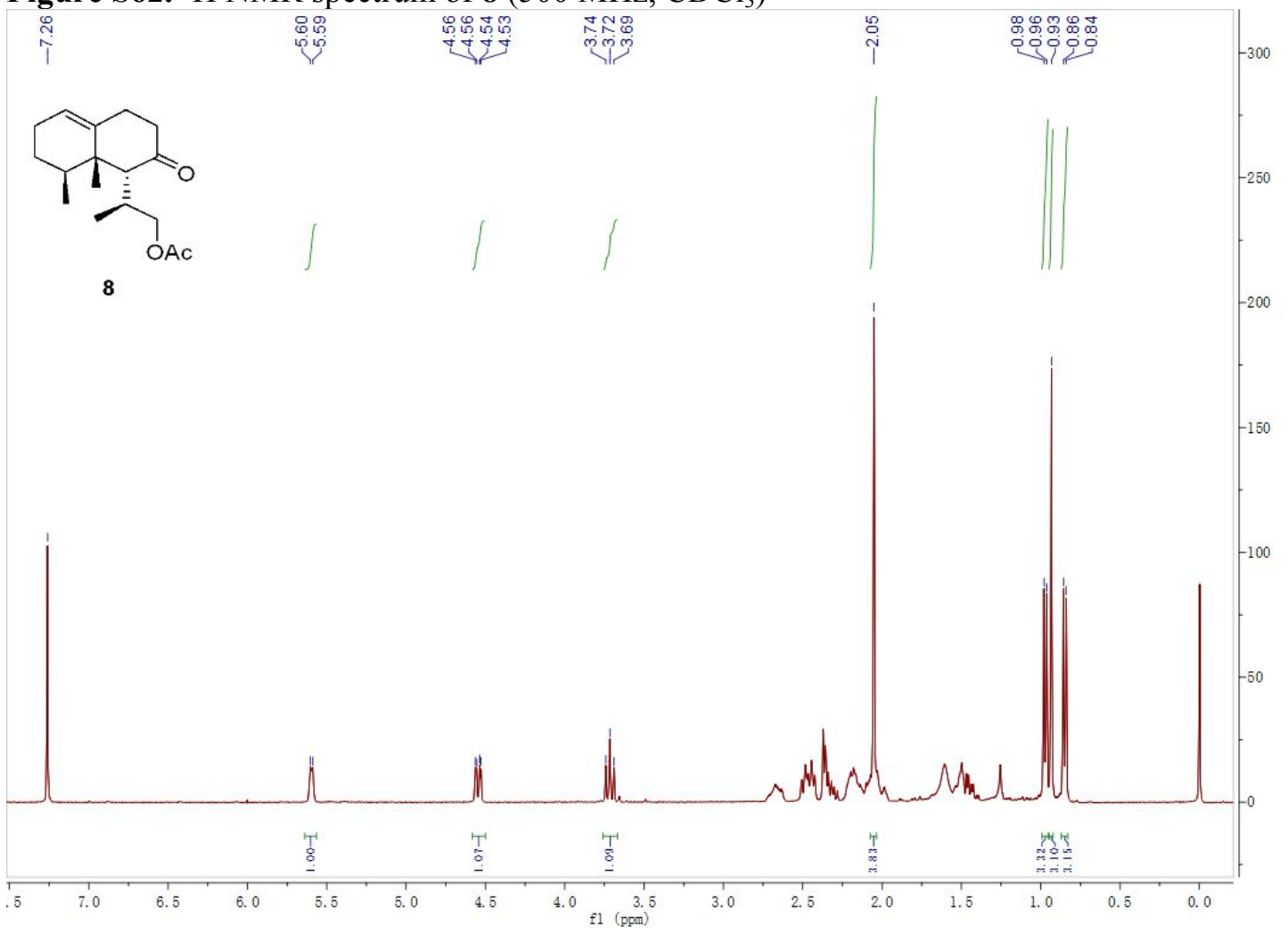


Figure S63. ${ }^{13} \mathrm{C}\left\{{ }^{1} \mathrm{H}\right\}$ NMR spectrum of $8\left(125 \mathrm{MHz}, \mathrm{CDCl}_{3}\right)$

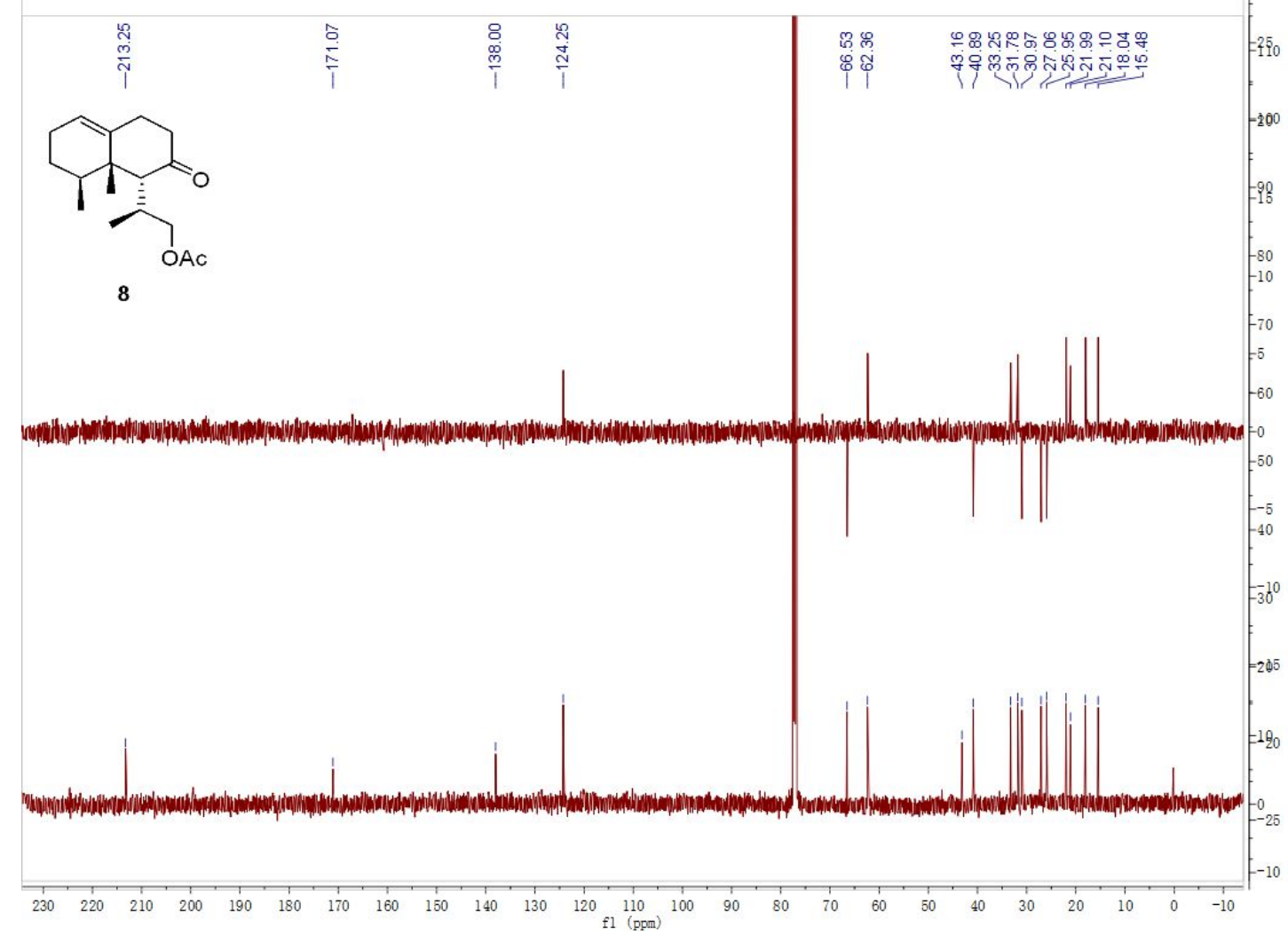

Figure S64. ${ }^{1} \mathrm{H}$ NMR spectrum of $9\left(500 \mathrm{MHz}, \mathrm{CDCl}_{3}\right)$

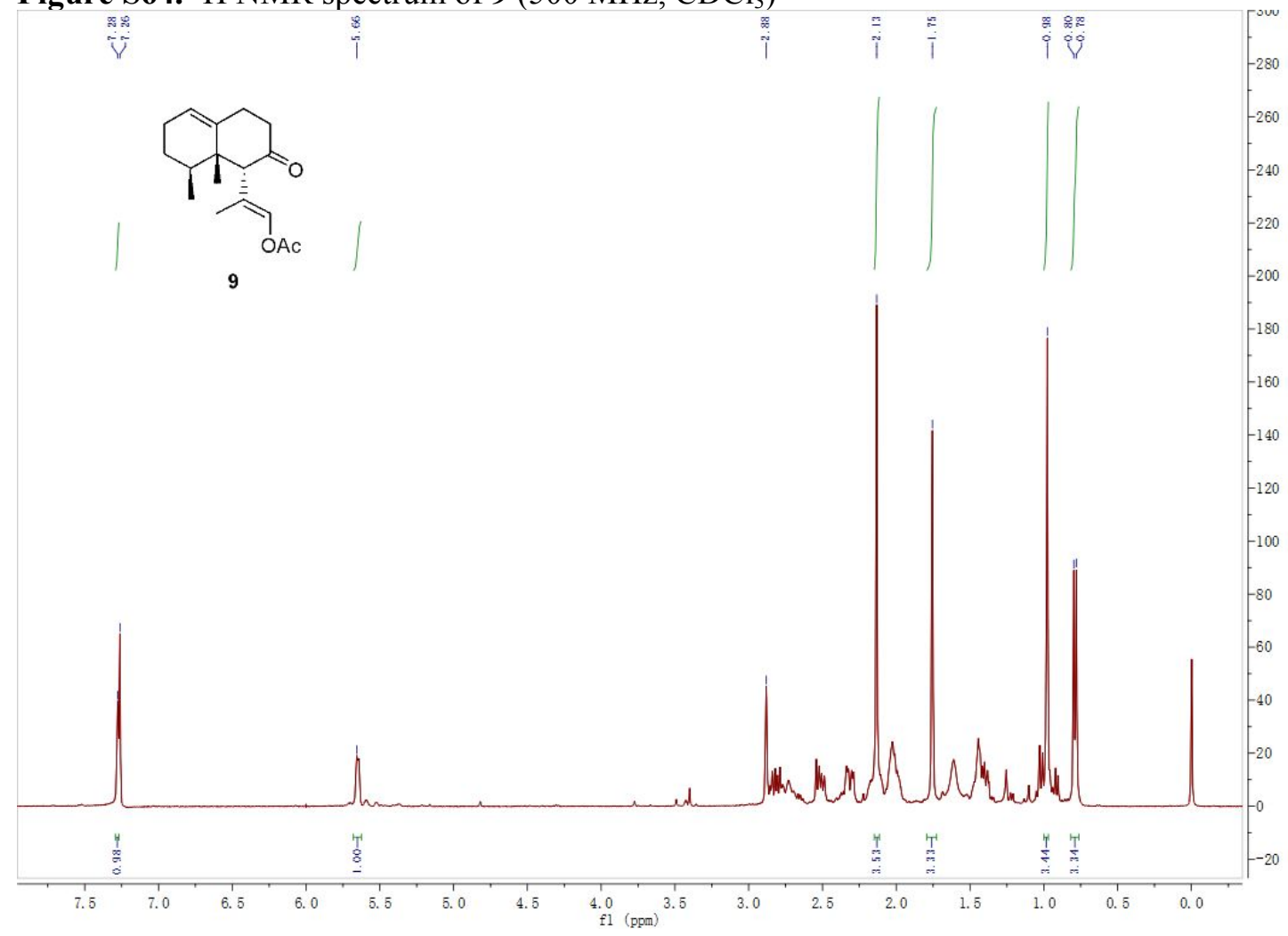


Figure S65. ${ }^{13} \mathrm{C}\left\{{ }^{1} \mathrm{H}\right\}$ NMR spectrum of $9\left(125 \mathrm{MHz}, \mathrm{CDCl}_{3}\right)$

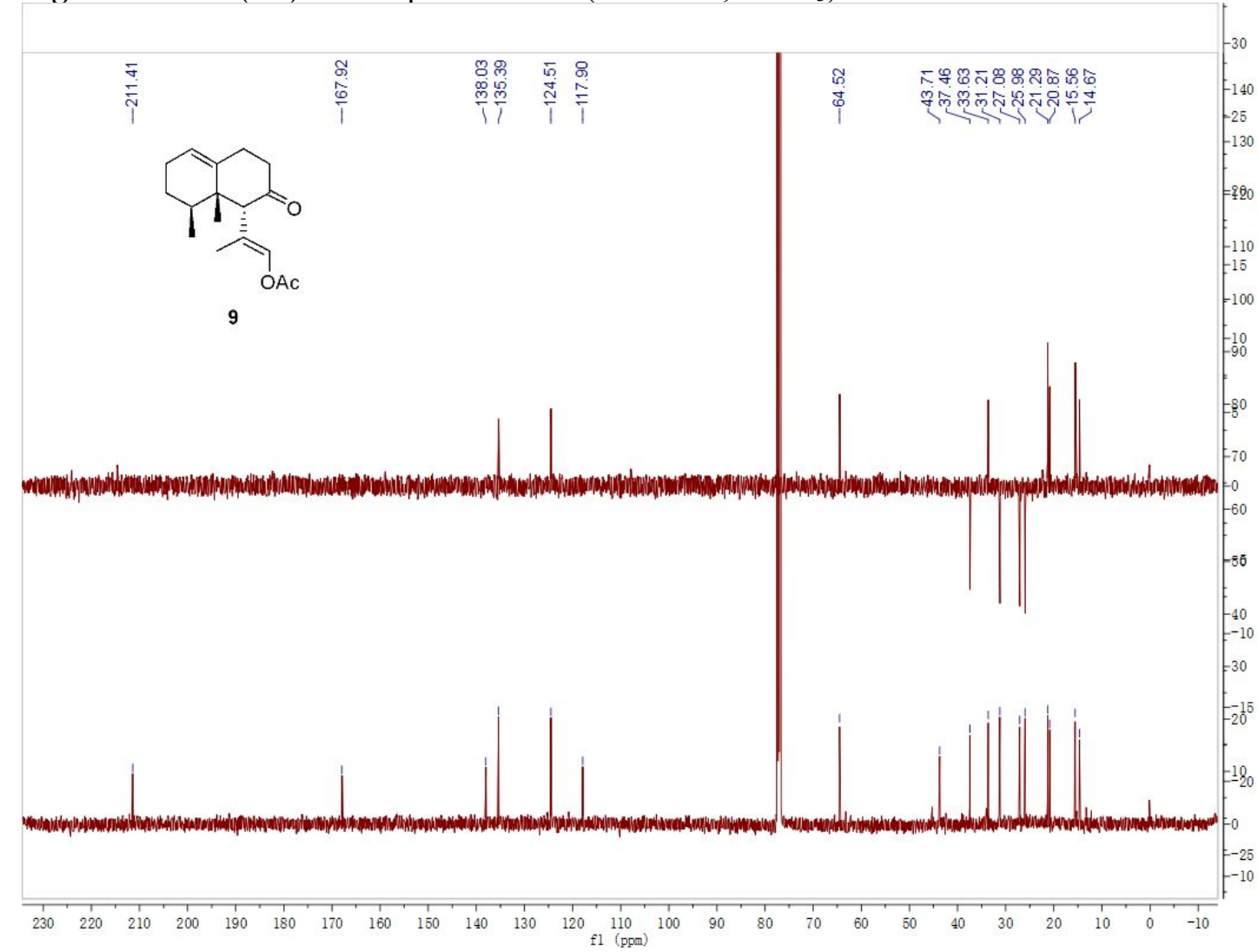

Figure S66. ${ }^{1} \mathrm{H}$ NMR spectrum of $10\left(500 \mathrm{MHz}, \mathrm{CDCl}_{3}\right)$

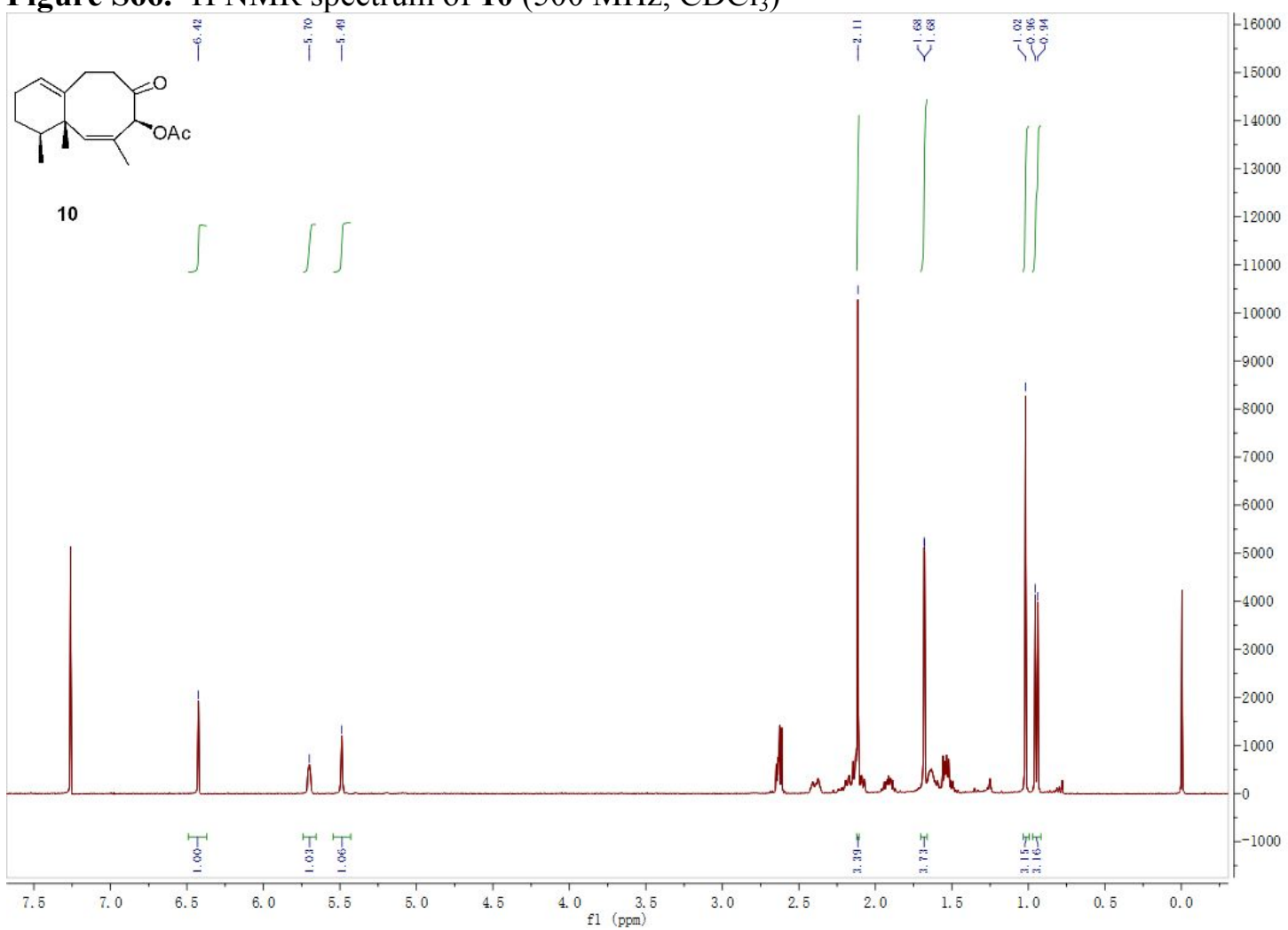


Figure S67. ${ }^{13} \mathrm{C}\left\{{ }^{1} \mathrm{H}\right\}$ NMR spectrum of $\mathbf{1 0}\left(125 \mathrm{MHz}, \mathrm{CDCl}_{3}\right)$

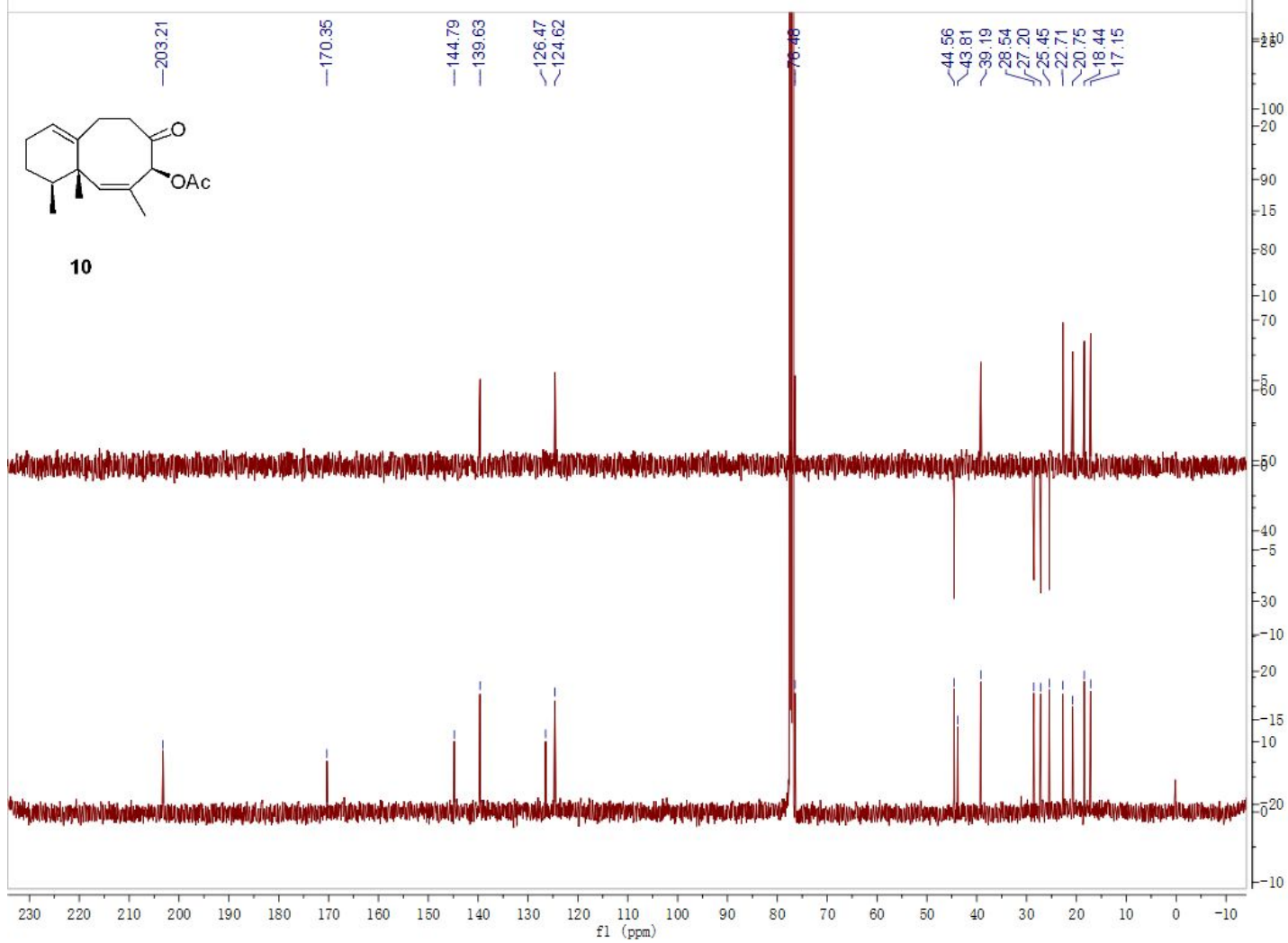

Figure S68. ${ }^{1} \mathrm{H}$ NMR spectrum of $11\left(400 \mathrm{MHz}, \mathrm{CDCl}_{3}\right)$

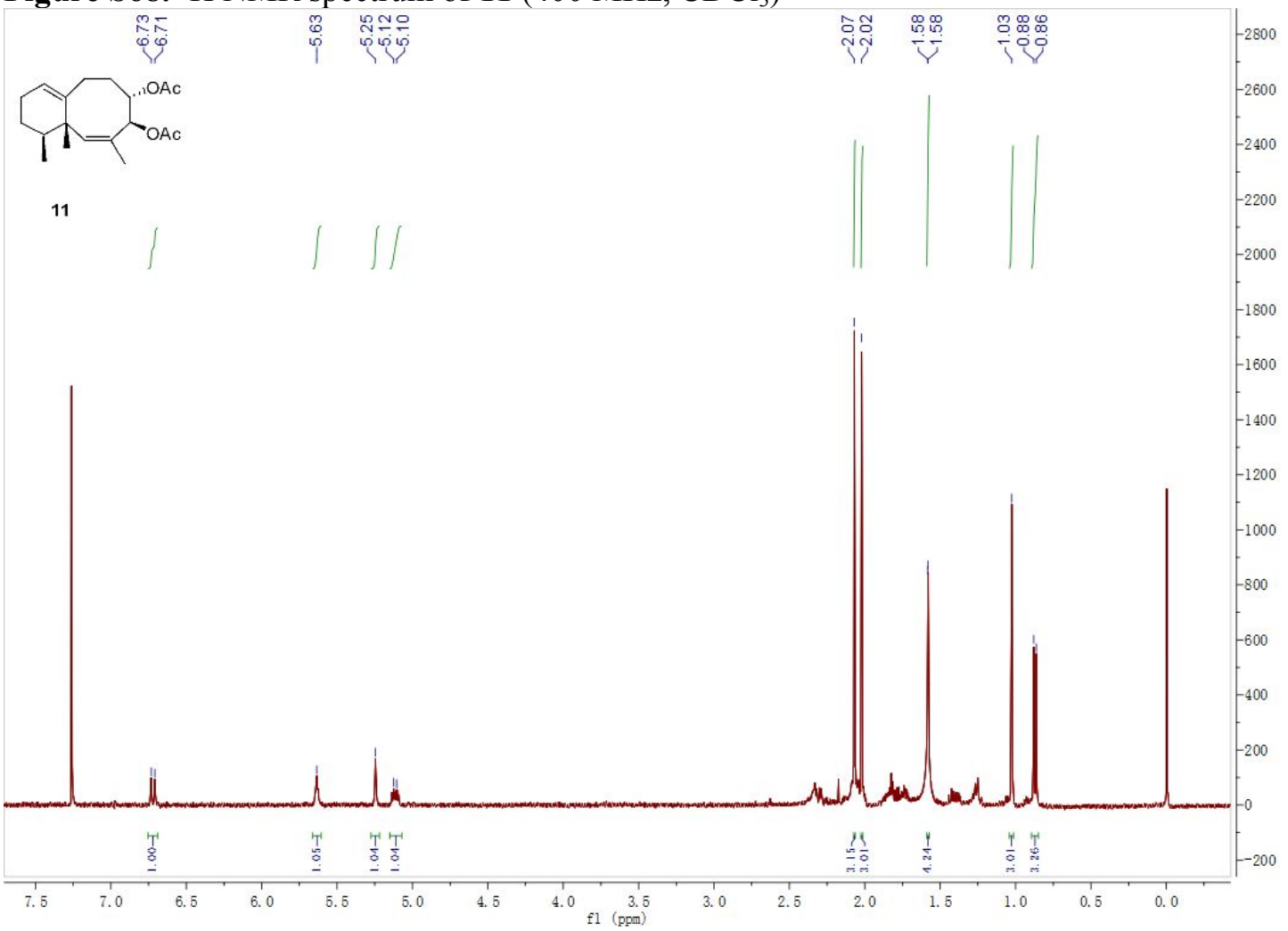




\section{Computational details}

All calculations were done following the general protocols previously described for DP4+. ${ }^{1,2}$ Conformational searches were done using de Macrocycle Conformational Sampling protocol in gas phase using the MMFF force field (as implemented in Macromodel). ${ }^{3}$ All conformers within $12 \mathrm{~kJ} / \mathrm{mol}$ of the lowest energy conformer were subjected to NMR calculations. DFT calculations were performed using Gaussian 09. ${ }^{4}$ Structure optimizations were done at the B3LYP/6-31G* level of theory. Magnetic shielding constants $(\sigma)$ were calculated by means of the gauge including atomic orbitals (GIAO) method, ${ }^{5}$ currently the most widely used to solve the gauge origin problem, at the mPW1PW91/6-31+G** level of theory as recommended for DP4+. Unscaled chemical shifts $\left(\delta_{\mathrm{u}}\right)$ were calculated using TMS as reference standard according to the following expression $\delta_{\mathrm{u}}=\sigma_{0^{-}} \sigma_{\mathrm{x}}$, where $\sigma_{\mathrm{x}}$ is the Boltzmann averaged shielding tensor (over all significantly populated conformations) and $\sigma_{0}$ is the shielding tensor of TMS computed at the same level of theory used to calculate $\sigma_{\mathrm{x}}$. Boltzmann averaging was done according to eq. S1:

$$
\sigma^{\mathrm{x}}=\frac{\sum_{i} \sigma_{i}^{\mathrm{x}} \mathrm{e}^{\left(-\mathrm{E}_{i} / \mathrm{RT}\right)}}{\sum_{i} \mathrm{e}^{\left(-\mathrm{E}_{i} / \mathrm{RT}\right)}}
$$

(Equation S1)

where $\sigma_{i}^{x}$ is the shielding constant for nucleus $\mathrm{x}$ in conformer $i$, $\mathrm{R}$ is the molar gas constant $\left(8.3145 \mathrm{~J} \mathrm{~K}^{-1} \mathrm{~mol}^{-1}\right)$, T is the temperature used for the calculation $(298 \mathrm{~K})$, and $E_{\mathrm{i}}$ is the relative energy of conformer $i$ (to the lowest energy conformer) obtained from a single-point NMR calculation at the corresponding level of theory. The scaled chemical shifts $\left(\delta_{\mathrm{S}}\right)$ were computed as $\delta_{\mathrm{S}}=\left(\delta_{\mathrm{u}}-\mathrm{b}\right) / \mathrm{m}$, where $\mathrm{m}$ and $\mathrm{b}$ are the slope and intercept, respectively, resulting from a linear regression calculation on a plot of $\delta_{\mathrm{u}}$ against $\delta_{\mathrm{exp}}$.

(1) Grimblat, N.; Zanardi, M. M.; Sarotti, A. M. Beyond DP4: An Improved Probability for the Stereochemical Assignment of Isomeric Compounds Using Quantum Chemical Calculations of NMR Shifts. J. Org. Chem. 2015, 80 (24), 12526-12534.

(2) Grimblat, N.; Gavín, J. A.; Hernández Daranas, A.; Sarotti, A. M. Combining the Power of J Coupling and DP4 Analysis on Stereochemical Assignments: The J-DP4 Methods. Org. Lett. 2019, 21 (11), 4003-4007.

(3) MacroModel Schrodinger release 2018-3; Schrodinger LLC: New York, 2018.

(4) Frisch, M. J.; Trucks, G. W.; Schlegel, H. B.; Scuseria, G. E.; Robb, M. A.; Cheeseman, J. R.; Scalmani, G.; Barone, V.; Mennucci, B.; Petersson, G. A.; Nakatsuji, H.; Caricato, M.; Li, X.; Hratchian, H. P.; Izmaylov, A. F.; Bloino, J.; Zheng, G.; Sonnenberg, J. L.; Hada, M.; Ehara, M.; Toyota, K.; Fukuda, R.; Hasegawa, J.; Ishida, M.; Nakajima, T.; Honda, Y.; Kitao, O.; Nakai, H.; Vreven, T.; Montgomery, J. A., Jr., Peralta, J. E.; Ogliaro, F.; Bearpark, M.; Heyd, J. J.; Brothers, E.; Kudin, K. N.; Staroverov, V. N.; Kobayashi, R.; Normand, J.; Raghavachari, K.; Rendell, A.; Burant, J. C.; Iyengar, S. S.; Tomasi, J.; Cossi, M.; Rega, N.; Millam, J. M.; Klene, M.; Knox, J. E.; Cross, J. B.; Bakken, V.; Adamo, C.; Jaramillo, J.; Gomperts, R.; Stratmann, R. E.; Yazyev, O.; Austin, A. J.; Cammi, R.; Pomelli, C.; Ochterski, J. W.; Martin, R. L.; Morokuma, K.; Zakrzewski, V. G.; Voth, G. A.; Salvador, P.; Dannenberg, J. J.; Dapprich, S.; Daniels, A. D.; Farkas, O.; Foresman, J. B.; Ortiz, J. V.; Cioslowski, J.; Fox, D. J. Gaussian 09, Gaussian, Inc.: Wallingford, CT, 2009.

(5) (a) Ditchfield, R. J. Chem. Phys. 1972, 56, 5688-5691. b) Ditchfield, R. Mol. Phys.1974, 27, 789-807. c) Rohlfing, C. M.; Allen, L. C.; Ditchfield, R. Chem. Phys. 1984, 87, 9-15. d) Wolinski, K.; Hinton, J. F.; Pulay, P. J. Am. Chem. Soc. 1990, 112, 8251-8260. 
Table S1. ${ }^{1} \mathrm{H}$ and ${ }^{13} \mathrm{C}$ NMR data of compounds $1,2,3,4$ and 5. ${ }^{\mathrm{a}}$

\begin{tabular}{|c|c|c|c|c|}
\hline \multirow{2}{*}{ No. } & \multicolumn{2}{|l|}{1} & \multicolumn{2}{|l|}{2} \\
\hline & $\delta_{\mathrm{H}}($ mult., $J$ in Hz) & $\delta_{\mathrm{C}}$ mult. & $\delta_{\mathrm{H}}$ (mult., $J$ in Hz) & $\delta_{\mathrm{C}}$ mult. \\
\hline 1 & $\begin{array}{l}1.81(\mathrm{dq}, 12.4,6.5,6.5,6.5, \\
6.5)\end{array}$ & $31.4(\mathrm{~d})$ & $1.82(\mathrm{~m})$ & $26.3(\mathrm{~d})$ \\
\hline 2 & 1.60 (overlapped) & $31.6(t)$ & $\begin{array}{l}2.04 \text { (ddd, 17.0, 6.3, } \\
6.3 \text { ) }\end{array}$ & $32.1(\mathrm{t})$ \\
\hline & $\begin{array}{l}0.51 \text { (dddd, 13.3, 13.3, 11.9, } \\
2.3)\end{array}$ & & 1.48 (overlapped) & \\
\hline 3 & $\begin{array}{l}1.33(\mathrm{~m}) \\
0.88 \text { (overlapped) }\end{array}$ & $25.5(\mathrm{t})$ & 5.14 (brd, 6.5) & 116.5 (d) \\
\hline 4 & $1.22(\mathrm{~m})$ & $43.0(\mathrm{~d})$ & - & $142.0(\mathrm{~s})$ \\
\hline 5 & $1.15(\mathrm{~d}, 2.5)$ & 36.3 (d) & $1.81(\mathrm{br} \mathrm{s})$ & 37.1 (d) \\
\hline 6 & - & $145.9(\mathrm{~s})$ & - & $153.7(\mathrm{~s})$ \\
\hline 7 & 4.89 (br s) & $120.4(d)$ & $\begin{array}{l}2.11(\mathrm{~m}) \\
1.61(\mathrm{dd}, 11.6,8.3)\end{array}$ & $28.9(\mathrm{t})$ \\
\hline 8 & $\begin{array}{l}2.51(\mathrm{dd}, 17.0,2.5) \\
2.15(\mathrm{dd}, 17.0,2.5)\end{array}$ & $40.4(\mathrm{t})$ & $\begin{array}{l}2.18(\mathrm{~m}) \\
2.12(\mathrm{~m})\end{array}$ & $29.1(\mathrm{t})$ \\
\hline 9 & - & $34.7(\mathrm{~s})$ & - & $40.0(\mathrm{~s})$ \\
\hline 10 & $0.21(t, 2.5)$ & 35.4 (d) & 1.25 (overlapped) & 25.7 (d) \\
\hline 11 & $1.46(\mathrm{~m})$ & 38.3 (d) & $2.18(\mathrm{~m})$ & 41.0 (d) \\
\hline 12 & $0.88(\mathrm{~d}, 6.7)$ & $16.4(\mathrm{q})$ & $1.03(\mathrm{~d}, 6.7)$ & $19.6(q)$ \\
\hline 13 & $\begin{array}{l}1.45(\mathrm{~m}) \\
1.18(\mathrm{~m})\end{array}$ & $34.5(t)$ & $\begin{array}{l}1.50(\mathrm{~m}) \\
1.40(\mathrm{~m})\end{array}$ & $31.8(t)$ \\
\hline 14 & $\begin{array}{l}2.03(\mathrm{~m}) \\
1.92(\mathrm{~m})\end{array}$ & $26.2(\mathrm{t})$ & $\begin{array}{l}1.50(\mathrm{~m}) \\
1.39(\mathrm{~m})\end{array}$ & $27.1(\mathrm{t})$ \\
\hline 15 & $5.11(\mathrm{t}, 7.1)$ & 125.3 (d) & $2.72(\mathrm{t}, 6.1)$ & 64.7 (d) \\
\hline 16 & - & $131.2(\mathrm{~s})$ & - & $58.4(\mathrm{~s})$ \\
\hline 17 & $1.69(\mathrm{~s})$ & $25.9(q)$ & $1.24(\mathrm{~s})$ & $18.7(\mathrm{q})$ \\
\hline 18 & $1.61(\mathrm{~s})$ & $17.8(\mathrm{q})$ & $1.30(\mathrm{~s})$ & $25.1(\mathrm{q})$ \\
\hline 19 & $0.92(\mathrm{~d}, 6.5)$ & $19.8(\mathrm{q})$ & $1.04(\mathrm{~d}, 6.7)$ & $19.2(\mathrm{q})$ \\
\hline 20 & $1.76(\mathrm{~d}, 1.3)$ & $16.8(\mathrm{q})$ & $\begin{array}{l}4.78 \text { (brs) } \\
4.62 \text { (brs) }\end{array}$ & $102.3(\mathrm{t})$ \\
\hline
\end{tabular}




\begin{tabular}{|c|c|c|c|c|c|c|}
\hline \multirow[b]{2}{*}{ No. } & \multicolumn{2}{|l|}{3} & \multicolumn{2}{|l|}{4} & \multicolumn{2}{|l|}{5} \\
\hline & $\delta_{\mathrm{H}}($ mult., $J$ in Hz) & $\delta_{\mathrm{C}}$ mult. & $\begin{array}{c}\delta_{\mathrm{H}} \text { (mult., } J \text { in } \\
\mathrm{Hz})\end{array}$ & $\delta_{\mathrm{C}}$ mult. & $\delta_{\mathrm{H}}$ (mult., $J$ in $\mathrm{Hz}$ ) & $\delta_{\mathrm{C}}$ mult. \\
\hline 1 & $1.77(\mathrm{~m})$ & $31.6(d)$ & $1.67(\mathrm{~m})$ & $31.5(\mathrm{~d})$ & $1.87(\mathrm{~m})$ & $25.9(d)$ \\
\hline \multirow[t]{2}{*}{2} & $1.36(\mathrm{~m})$ & $25.5(\mathrm{t})$ & $1.35(\mathrm{~m})$ & $25.5(\mathrm{t})$ & $2.13(\mathrm{~m})$ & $31.7(\mathrm{t})$ \\
\hline & $\begin{array}{c}0.76 \text { (dddd, 13.7, 13.7 } \\
11.9,2.1)\end{array}$ & & $0.73(\mathrm{~m})$ & & $\begin{array}{c}1.48(\mathrm{ddd}, 17.2 \\
11.6,2.1)\end{array}$ & \\
\hline 3 & $\begin{array}{c}1.84(\mathrm{ddd}, 14.1,4.8,1.9) \\
0.97 \text { (overlapped) }\end{array}$ & $22.3(\mathrm{t})$ & $\begin{array}{l}1.83(\mathrm{~m}) \\
0.94(\mathrm{~m})\end{array}$ & $22.1(\mathrm{t})$ & $5.44(\mathrm{dd}, 6.9,0.5)$ & $114.6(d)$ \\
\hline 4 & - & $74.5(\mathrm{~s})$ & - & $74.8(\mathrm{~d})$ & - & $142.9(\mathrm{~s})$ \\
\hline 5 & $2.00(\mathrm{~d}, 3.0)$ & $29.3(d)$ & $1.38(\mathrm{~d}, 3.2)$ & $27.8(d)$ & 1.80 (br s) & $37.4(\mathrm{~d})$ \\
\hline 6 & - & $155.0(\mathrm{~s})$ & - & $84.9(\mathrm{~s})$ & - & $153.6(\mathrm{~s})$ \\
\hline \multirow[t]{2}{*}{7} & $2.17(\mathrm{~m})$ & $29.2(\mathrm{t})$ & $1.50(\mathrm{~m})$ & $35.4(\mathrm{t})$ & $2.21(\mathrm{~m})$ & $28.8(\mathrm{t})$ \\
\hline & $2.00(\mathrm{~m})$ & & $1.45(\mathrm{~m})$ & & $2.14(\mathrm{~m})$ & \\
\hline \multirow[t]{2}{*}{8} & $1.58(\mathrm{~m})$ & $30.2(\mathrm{t})$ & $1.85(\mathrm{~m})$ & $28.3(\mathrm{t})$ & $2.14(\mathrm{~m})$ & $29.1(\mathrm{t})$ \\
\hline & $1.58(\mathrm{~m})$ & & $1.55(\mathrm{~m})$ & & $1.66(\mathrm{~m})$ & \\
\hline 9 & - & $38.2(\mathrm{~s})$ & - & $34.5(\mathrm{~s})$ & - & $40.1(\mathrm{~s})$ \\
\hline 10 & $1.10(\mathrm{~d}, 3.0)$ & 35.9 (d) & $1.12(\mathrm{~d}, 3.2)$ & 30.9 (d) & $1.35(\mathrm{~d}, 2.7)$ & $25.1(\mathrm{~d})$ \\
\hline 11 & $1.61(\mathrm{~m})$ & $41.6(d)$ & $1.69(\mathrm{~m})$ & $41.6(d)$ & - & $85.2(\mathrm{~s})$ \\
\hline 12 & $0.89(\mathrm{~d}, 6.7)$ & $17.3(q)$ & $0.90(\mathrm{~d}, 6.6)$ & $17.3(q)$ & $1.34(\mathrm{~s})$ & $27.1(q)$ \\
\hline \multirow[t]{2}{*}{13} & $1.59(\mathrm{~m})$ & $28.0(\mathrm{t})$ & $1.60(\mathrm{~m})$ & $28.0(\mathrm{t})$ & $\begin{array}{c}2.06(\mathrm{ddd}, 11.7 \\
8.3,8.3)\end{array}$ & $37.3(\mathrm{t})$ \\
\hline & $1.36(\mathrm{~m})$ & & $1.36(\mathrm{~m})$ & & $\begin{array}{c}1.76(\mathrm{ddd}, 11.7 \\
8.3,8.3)\end{array}$ & \\
\hline \multirow[t]{2}{*}{14} & $1.54(\mathrm{~m})$ & $25.8(\mathrm{t})$ & $1.55(\mathrm{~m})$ & $26.0(\mathrm{t})$ & $1.82(\mathrm{~m})$ & $26.6(t)$ \\
\hline & $1.38(\mathrm{~m})$ & & $1.35(\mathrm{~m})$ & & $1.82(\mathrm{~m})$ & \\
\hline 15 & $3.20(\mathrm{dd}, 11.1,2.6)$ & $75.3(d)$ & $\begin{array}{l}3.21(\mathrm{dd} \\
11.4,2.5)\end{array}$ & $75.4(d)$ & $3.78(\mathrm{dd}, 7.4,7.4)$ & $86.3(d)$ \\
\hline 16 & - & $72.1(\mathrm{~s})$ & - & $72.2(\mathrm{~s})$ & - & $71.1(\mathrm{~s})$ \\
\hline 17 & $1.13(\mathrm{~s})$ & $24.3(q)$ & $1.11(\mathrm{~s})$ & $23.9(q)$ & $1.13(\mathrm{~s})$ & 24.3 (q) \\
\hline 18 & $1.20(\mathrm{~s})$ & $27.2(q)$ & $1.17(\mathrm{~s})$ & $26.9(\mathrm{q})$ & $1.23(\mathrm{~s})$ & $27.5(q)$ \\
\hline 19 & $0.95(\mathrm{~d}, 6.6)$ & $18.9(q)$ & $0.93(\mathrm{~d}, 6.6)$ & $18.8(q)$ & $1.04(\mathrm{~d}, 6.5)$ & $19.2(q)$ \\
\hline \multirow[t]{2}{*}{20} & 4.83 (brs) & $102.1(\mathrm{t})$ & $1.25(\mathrm{~s})$ & $24.3(\mathrm{q})$ & 4.78 (brs) & $102.3(\mathrm{t})$ \\
\hline & 4.62 (brs) & & & & 4.63 (brs) & \\
\hline $\mathrm{OMe}$ & & & $3.35(\mathrm{~s})$ & $51.7(q)$ & & \\
\hline
\end{tabular}

${ }^{\text {a }}{ }^{1} \mathrm{H}(500 \mathrm{MHz})$ and ${ }^{13} \mathrm{C}(125 \mathrm{MHz})$ data in $\mathrm{CDCl}_{3}$. 


\subsection{Structures of isomers studied for compounds 1-5 and isoelisabethatriene}

Figure S69. Structures of isomers 1-8 of compound 1

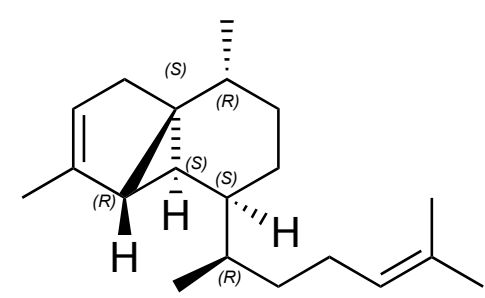

Isomer 1

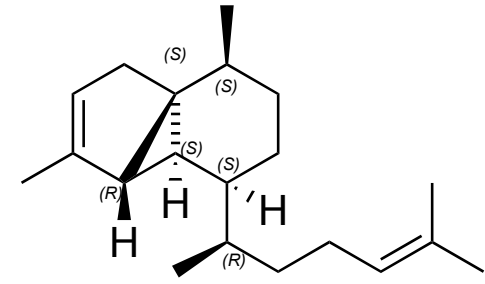

Isomer 3
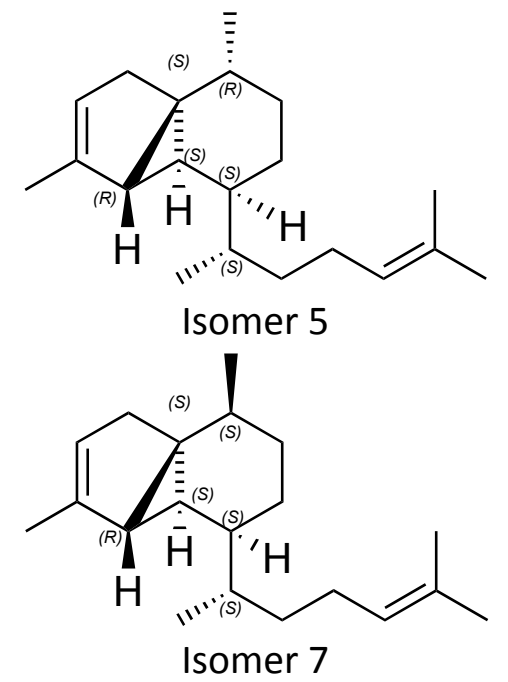

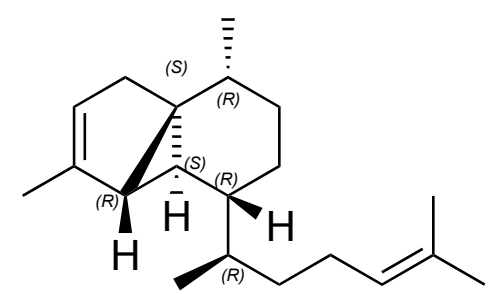

Isomer 2

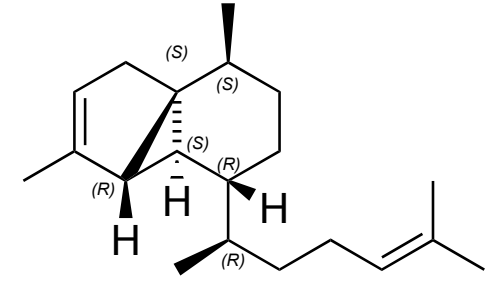

Isomer 4
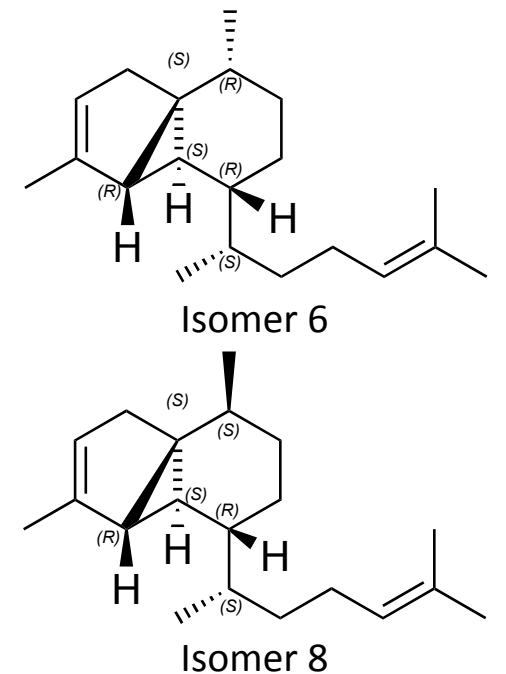
Figure S70. Structures of isomers 1-8 of compound 2

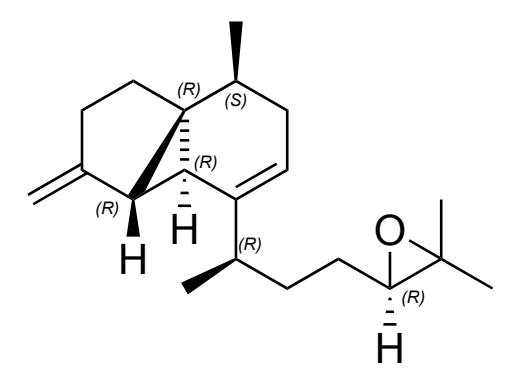

Isomer 1

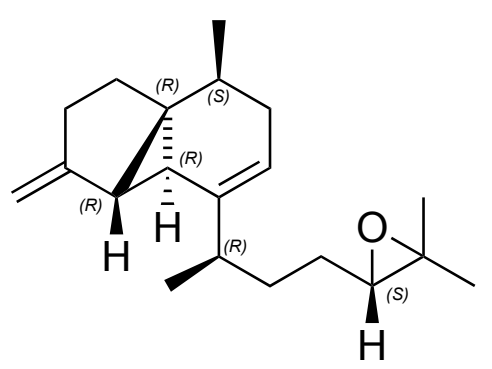

Isomer 5

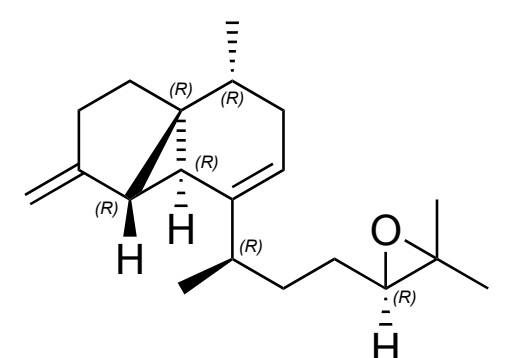

Isomer 2

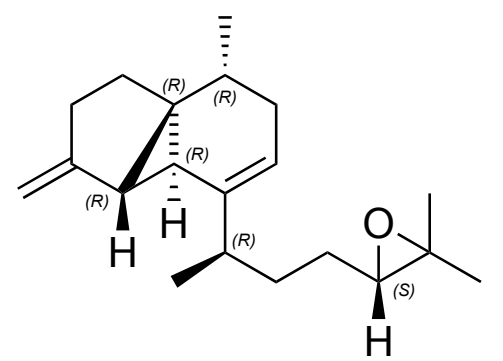

Isomer 6
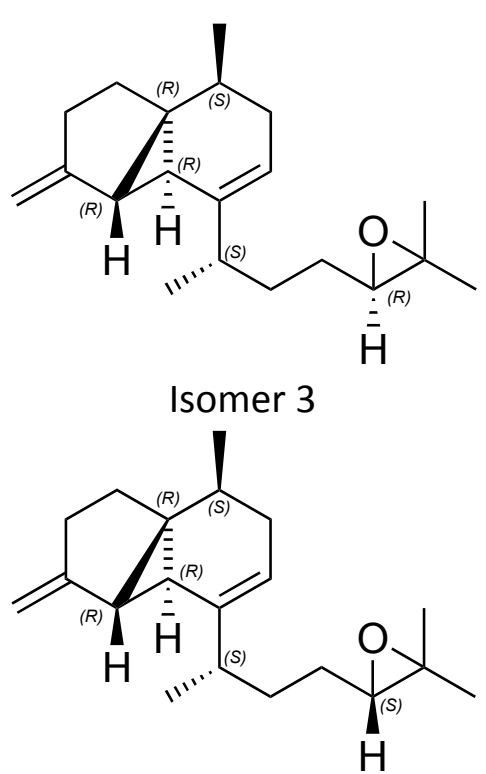

Isomer 7

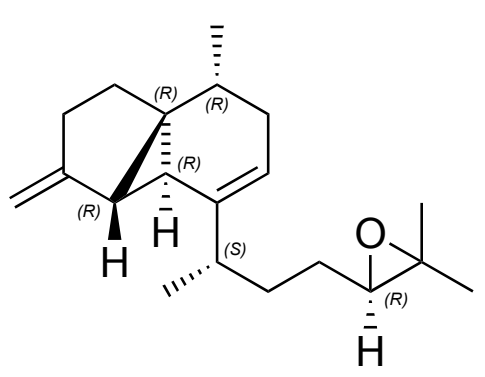

Isomer 4

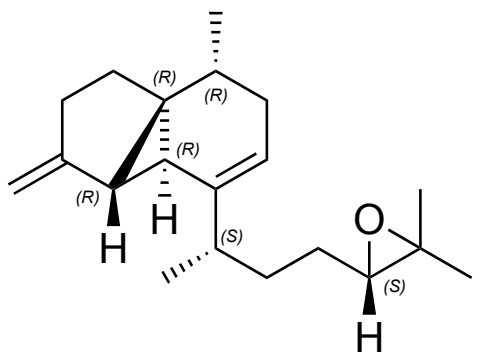

Isomer 8

*Isomers that are not compatible with NOESY experimental data are shown in red 
Figure S71. Structures of isomers 1-16 of compound 3
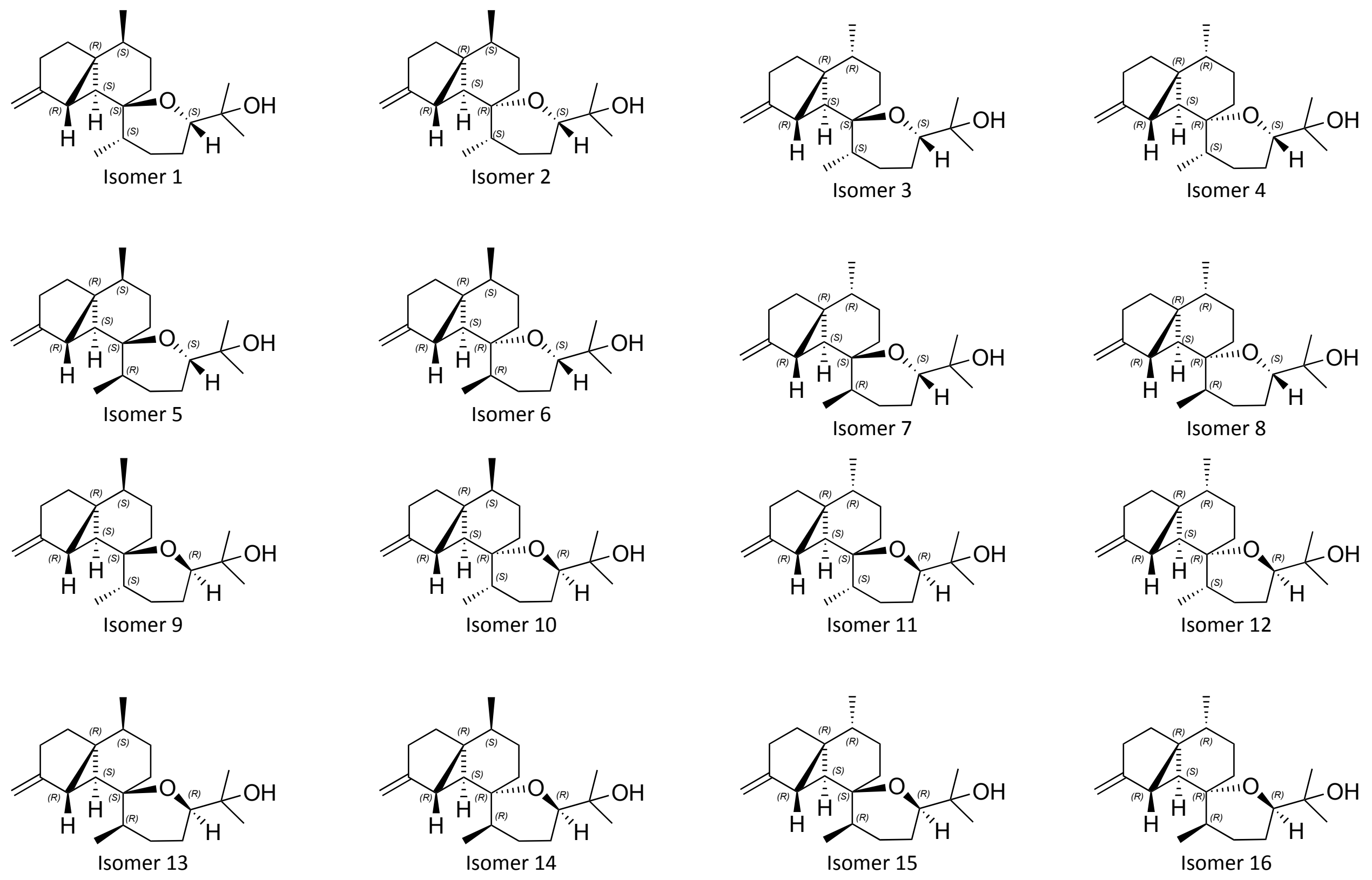
Figure S72. Structures of isomers 1-32 of compound 4
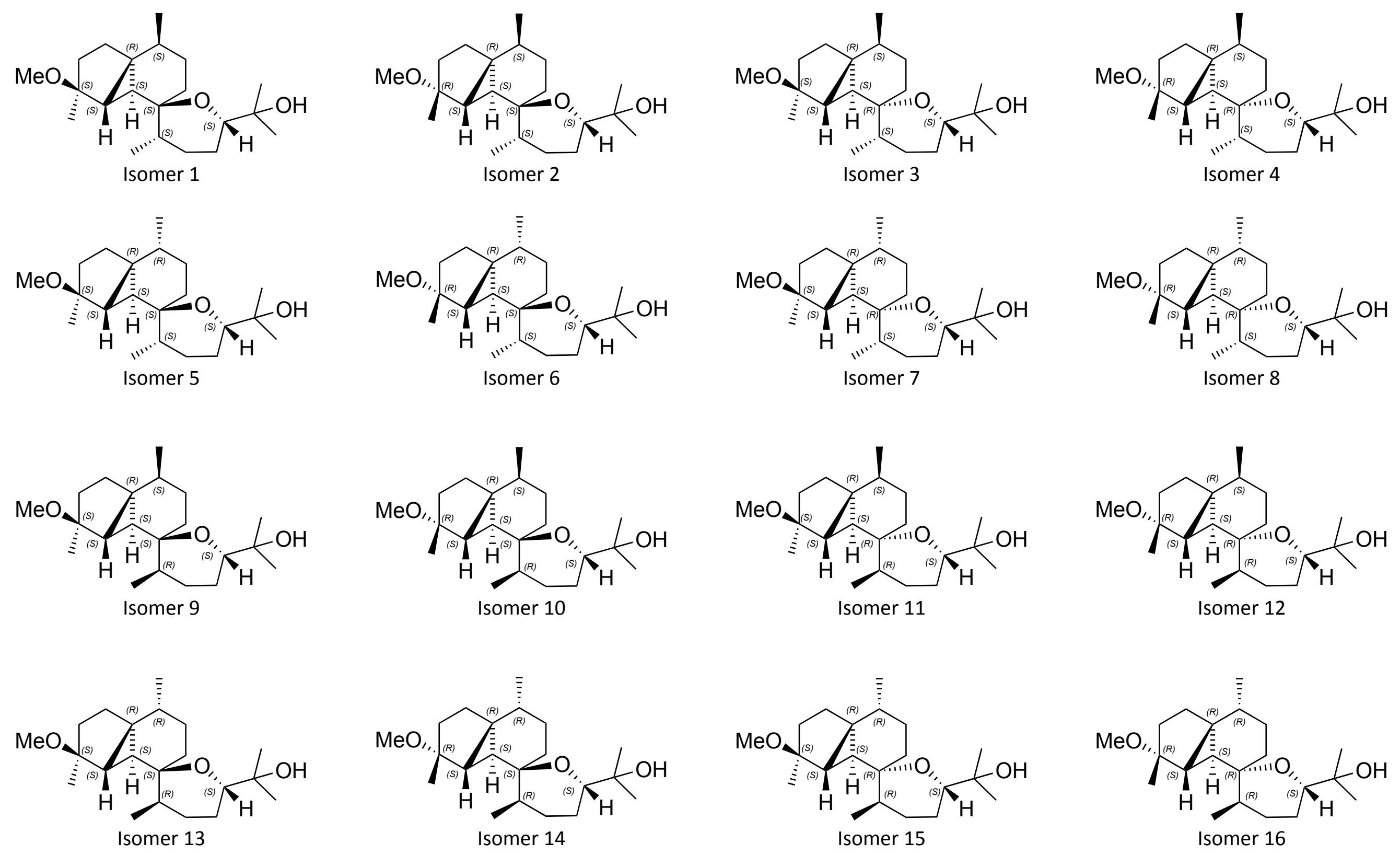


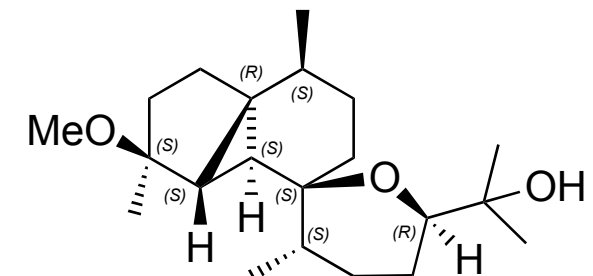

Isomer 17

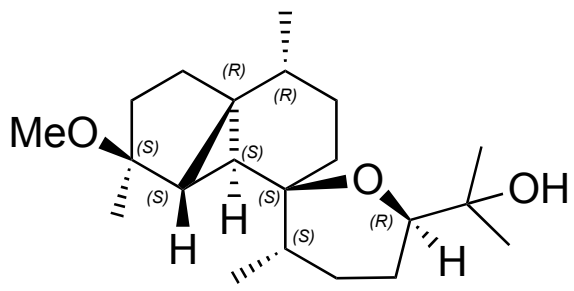

Isomer 21

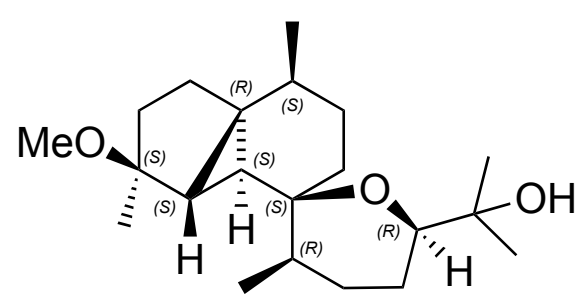

Isomer 25

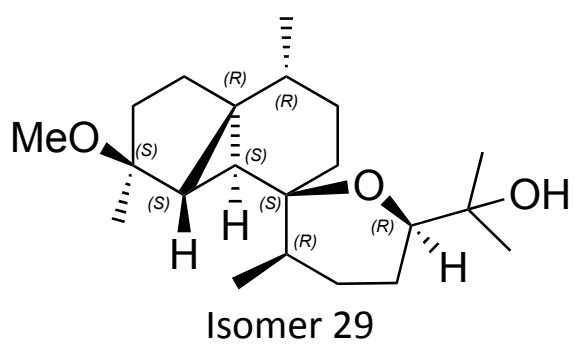

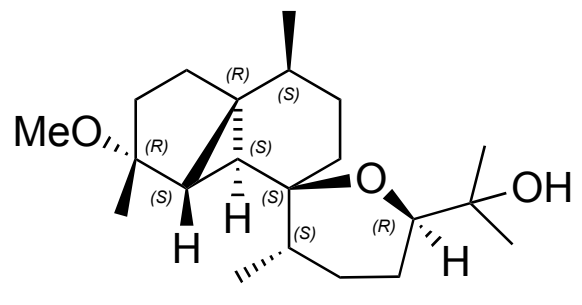

Isomer 18
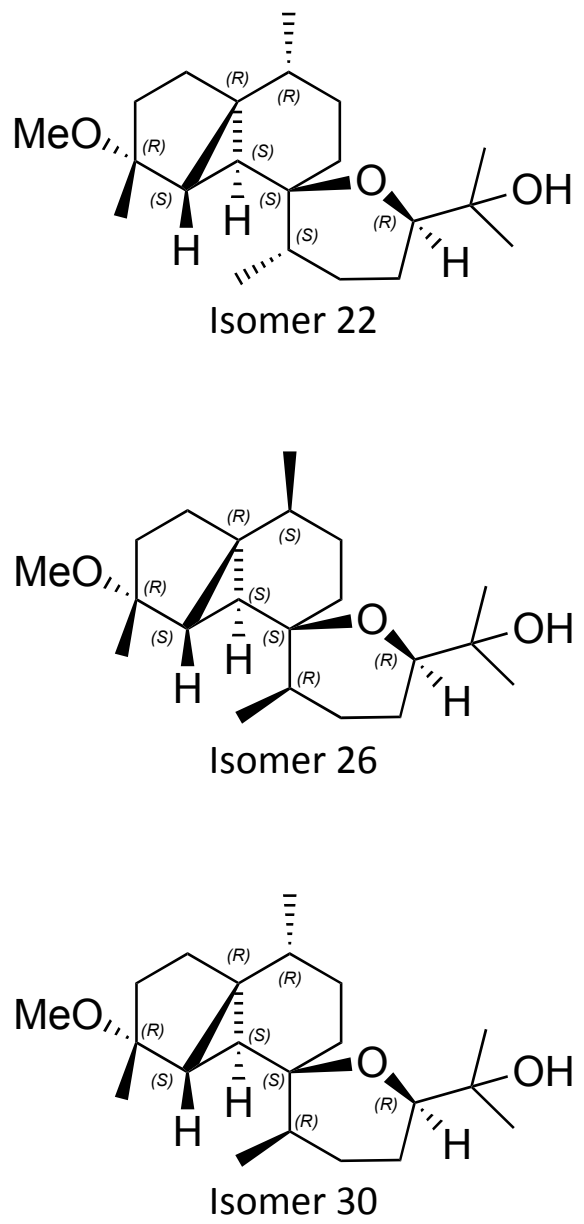
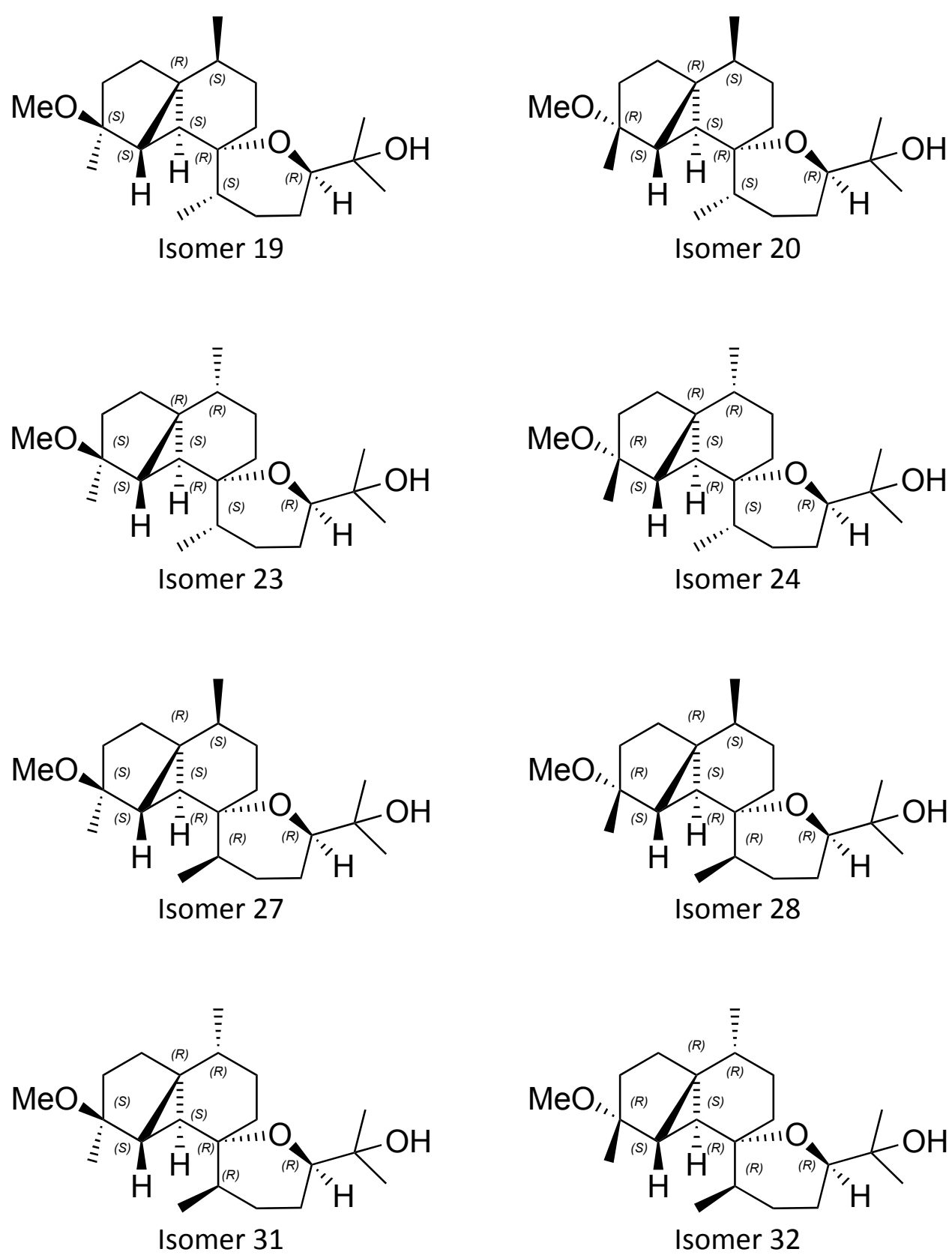


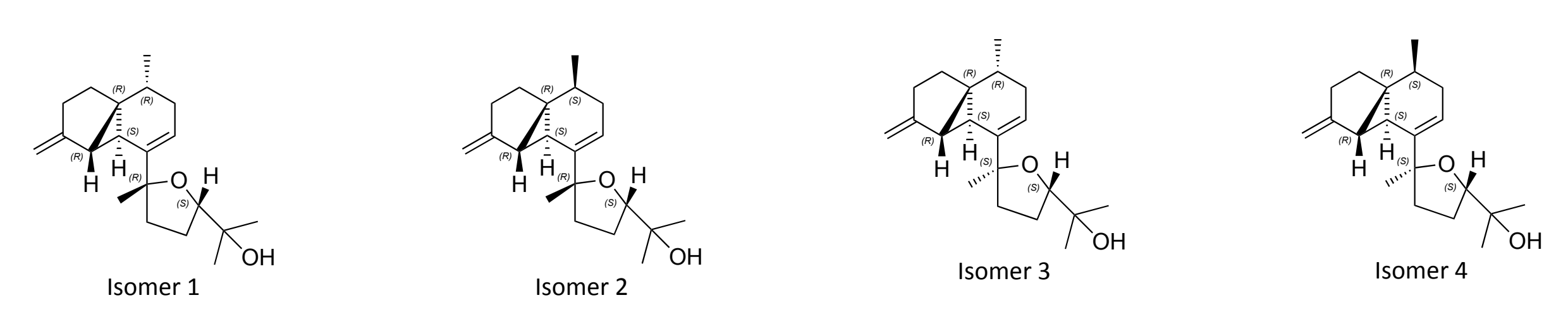

程独 
Figure S74. Structures of isomers 1-4 of compound isoelisabethatriene

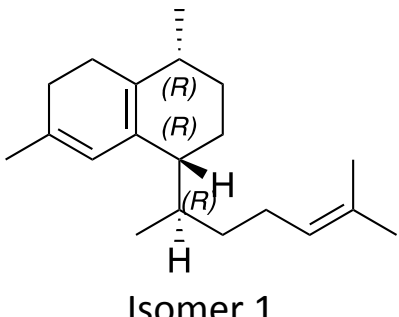

Isomer 1

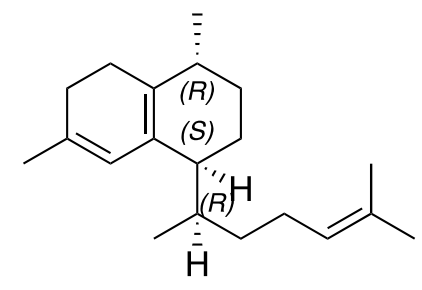

Isomer 2

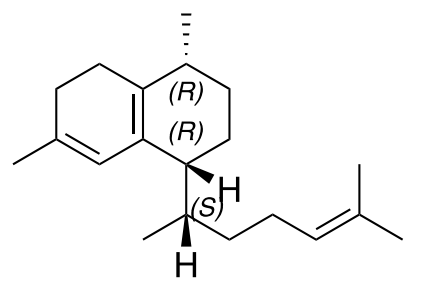

Isomer 3

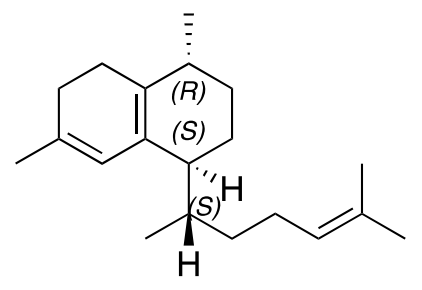

Isomer 4 


\subsection{Isotropic magnetic shielding constants of compounds 1-5 and isoelisabethatriene}

Table S2. Boltzmann averaged GIAO isotropic magnetic shielding constants $(\sigma)$ of isomers 1-8 calculated at the PCM/mPW1PW91/6-31+G**//B3LYP/6-31G* level of theory for compound 1

Isotropic shielding constants

\begin{tabular}{|c|c|c|c|c|c|c|c|c|}
\hline Nuclei & Isomer 1 & Isomer 2 & Isomer 3 & Isomer 4 & Isomer 5 & Isomer 6 & Isomer 7 & Isomer 8 \\
\hline C 1 & 161.33 & 162.55 & 162.59 & 161.30 & 161.12 & 162.95 & 162.25 & 161.01 \\
\hline$C 2$ & 159.42 & 164.94 & 163.11 & 162.57 & 159.72 & 164.81 & 162.93 & 162.37 \\
\hline C 3 & 169.66 & 173.39 & 172.31 & 167.27 & 169.95 & 174.26 & 171.92 & 167.83 \\
\hline $\mathrm{C} 4$ & 153.95 & 152.07 & 153.65 & 150.95 & 154.72 & 151.38 & 153.77 & 149.60 \\
\hline$C 5$ & 156.10 & 152.97 & 162.34 & 156.04 & 155.99 & 153.89 & 161.88 & 156.38 \\
\hline C 6 & 50.55 & 49.94 & 49.66 & 49.84 & 50.83 & 49.42 & 49.75 & 49.56 \\
\hline$C 7$ & 76.26 & 76.50 & 76.03 & 76.19 & 76.24 & 76.56 & 76.02 & 76.24 \\
\hline C 8 & 154.82 & 152.16 & 149.84 & 152.64 & 154.21 & 152.05 & 150.32 & 152.82 \\
\hline$C 9$ & 159.86 & 157.81 & 158.45 & 157.55 & 160.09 & 157.76 & 158.95 & 157.92 \\
\hline C 10 & 156.99 & 160.08 & 158.08 & 158.23 & 157.30 & 159.23 & 158.72 & 157.66 \\
\hline C 11 & 155.49 & 155.52 & 155.92 & 156.04 & 155.71 & 156.12 & 155.48 & 155.75 \\
\hline C 12 & 178.51 & 178.53 & 178.35 & 178.50 & 178.22 & 179.46 & 178.72 & 178.94 \\
\hline C 13 & 160.16 & 161.07 & 159.93 & 160.48 & 160.09 & 160.09 & 159.60 & 160.58 \\
\hline C 14 & 167.53 & 166.56 & 167.31 & 166.86 & 167.80 & 166.38 & 167.44 & 166.48 \\
\hline C 15 & 71.17 & 71.18 & 71.25 & 71.14 & 70.91 & 71.34 & 71.10 & 71.35 \\
\hline C 16 & 64.53 & 64.04 & 64.41 & 64.13 & 64.81 & 63.81 & 64.65 & 63.93 \\
\hline C 17 & 169.12 & 168.88 & 169.00 & 169.08 & 168.96 & 169.07 & 169.04 & 169.10 \\
\hline C 18 & 177.84 & 177.60 & 177.87 & 177.71 & 177.89 & 177.64 & 178.02 & 177.69 \\
\hline C 19 & 175.49 & 177.56 & 177.12 & 175.92 & 175.72 & 177.56 & 176.64 & 176.18 \\
\hline C 20 & 177.35 & 177.52 & 177.45 & 177.41 & 177.41 & 177.60 & 177.28 & 177.55 \\
\hline H 1 & 29.67 & 29.52 & 29.60 & 29.71 & 29.68 & 29.50 & 29.60 & 29.75 \\
\hline $\mathrm{H} 2 \mathrm{a}$ & 30.10 & 30.26 & 30.09 & 30.01 & 30.10 & 30.26 & 30.04 & 30.01 \\
\hline $\mathrm{H} 2 \mathrm{~b}$ & 30.53 & 30.28 & 30.47 & 30.91 & 30.51 & 30.28 & 30.43 & 30.89 \\
\hline H За & 30.08 & 30.25 & 30.26 & 30.31 & 30.09 & 30.25 & 30.32 & 30.30 \\
\hline $\mathrm{H} 3 \mathrm{~b}$ & 30.77 & 30.61 & 30.64 & 30.51 & 30.72 & 30.63 & 30.51 & 30.42 \\
\hline H 4 & 29.89 & 30.02 & 29.98 & 30.30 & 29.86 & 30.05 & 29.94 & 30.28 \\
\hline H 5 & 30.16 & 30.21 & 29.99 & 30.20 & 30.09 & 30.23 & 29.93 & 30.25 \\
\hline H 7 & 26.30 & 26.29 & 26.25 & 26.27 & 26.28 & 26.29 & 26.23 & 26.25 \\
\hline H 8a & 28.78 & 29.09 & 29.03 & 28.93 & 28.80 & 29.07 & 29.07 & 28.92 \\
\hline $\mathrm{H} 8 \mathrm{~b}$ & 29.28 & 29.20 & 29.13 & 29.32 & 29.26 & 29.18 & 29.14 & 29.28 \\
\hline H 10 & 31.12 & 31.35 & 31.01 & 31.31 & 31.15 & 31.31 & 31.01 & 31.29 \\
\hline H 11 & 30.07 & 30.03 & 30.09 & 30.03 & 30.05 & 30.04 & 30.02 & 30.07 \\
\hline H $12 a-c$ & 30.64 & 30.65 & 30.63 & 30.66 & 30.55 & 30.65 & 30.55 & 30.62 \\
\hline H 13a & 30.08 & 30.07 & 30.09 & 30.08 & 30.05 & 30.15 & 30.11 & 30.13 \\
\hline $\mathrm{H} 13 \mathrm{~b}$ & 30.19 & 30.35 & 30.17 & 30.40 & 30.35 & 30.24 & 30.27 & 30.29 \\
\hline H 14a & 29.35 & 29.42 & 29.39 & 29.40 & 29.43 & 29.37 & 29.46 & 29.43 \\
\hline $\mathrm{H} 14 \mathrm{~b}$ & 29.56 & 29.46 & 29.52 & 29.49 & 29.53 & 29.52 & 29.48 & 29.44 \\
\hline H 15 & 26.10 & 26.09 & 26.05 & 26.11 & 26.09 & 26.08 & 26.05 & 26.09 \\
\hline H 17a-c & 29.84 & 29.83 & 29.85 & 29.83 & 29.85 & 29.83 & 29.85 & 29.85 \\
\hline H 18a-c & 29.86 & 29.88 & 29.87 & 29.89 & 29.87 & 29.88 & 29.89 & 29.87 \\
\hline H 19a-c & 30.57 & 30.54 & 30.76 & 30.70 & 30.56 & 30.53 & 30.75 & 30.72 \\
\hline H 20a-c & 29.72 & 29.73 & 29.73 & 29.73 & 29.71 & 29.77 & 29.75 & 29.76 \\
\hline
\end{tabular}


Table S3. Boltzmann averaged GIAO isotropic magnetic shielding constants $(\sigma)$ of isomers 1-8 calculated at the PCM/mPW1PW91/6-31+G**//B3LYP/6-31G* level of theory for compound 2

\begin{tabular}{|c|c|c|c|c|c|c|c|c|}
\hline \multicolumn{9}{|c|}{ Isotropic shielding constants } \\
\hline Nuclei & Isomer 1 & Isomer 2 & Isomer 3 & Isomer 4 & Isomer 5 & Isomer 6 & Isomer 7 & Isomer 8 \\
\hline C 1 & 166.72 & 164.48 & 166.48 & 164.65 & 166.49 & 164.71 & 166.13 & 164.89 \\
\hline $\mathrm{C} 2$ & 160.41 & 161.99 & 160.36 & 162.26 & 160.84 & 162.27 & 161.08 & 161.86 \\
\hline C 3 & 79.97 & 82.46 & 79.37 & 81.8 & 79.57 & 81.67 & 79.11 & 82.9 \\
\hline C 4 & 52.1 & 51.45 & 51.97 & 53.12 & 52.56 & 52.53 & 53.08 & 51.99 \\
\hline $\mathrm{C} 5$ & 154.14 & 152.35 & 154.33 & 151.64 & 154.12 & 152.09 & 153.86 & 151.65 \\
\hline C 6 & 41.91 & 42.3 & 41.79 & 42.05 & 41.6 & 41.92 & 42.35 & 42.07 \\
\hline C 7 & 164.69 & 164.37 & 164.66 & 164.74 & 165.08 & 164.52 & 164.99 & 164.77 \\
\hline C 8 & 166.01 & 162.7 & 164.77 & 162.32 & 165.4 & 162.16 & 164.91 & 162.08 \\
\hline C 9 & 151.47 & 151.79 & 151.32 & 151.68 & 151.48 & 151.85 & 151.33 & 151.42 \\
\hline C 10 & 168.79 & 169.67 & 167.14 & 168.31 & 167.76 & 167.4 & 167.14 & 168.6 \\
\hline C 11 & 151.1 & 150.83 & 150.86 & 150.74 & 151.09 & 151.06 & 150.69 & 151.65 \\
\hline C 12 & 174.37 & 174.6 & 174.81 & 174.84 & 174.96 & 174.42 & 175.54 & 175.74 \\
\hline C 13 & 162 & 162.71 & 163.07 & 163.19 & 162.97 & 163.34 & 164.15 & 163.94 \\
\hline C 14 & 165.45 & 165.5 & 165.51 & 165.25 & 165.56 & 165.65 & 165.55 & 165.97 \\
\hline C 15 & 130.84 & 130.82 & 131.02 & 130.63 & 131.06 & 130.94 & 131.4 & 131.13 \\
\hline C 16 & 136.65 & 136.47 & 136.39 & 136.31 & 136.41 & 136.43 & 136.47 & 136.44 \\
\hline C 17 & 176.52 & 176.2 & 177.06 & 176.83 & 170.94 & 170.87 & 170.76 & 170.68 \\
\hline C 18 & 170.86 & 170.94 & 170.72 & 170.85 & 177.04 & 176.88 & 177.06 & 176.96 \\
\hline C 19 & 176.7 & 177.37 & 176.68 & 176.54 & 176.62 & 176.99 & 176.44 & 176.6 \\
\hline C 20 & 96.81 & 96.42 & 96.94 & 96.73 & 96.88 & 96.48 & 97.24 & 97 \\
\hline H 1 & 29.68 & 29.5 & 29.69 & 29.49 & 29.69 & 29.52 & 29.7 & 29.43 \\
\hline $\mathrm{H} 2 \mathrm{a}$ & 29.72 & 29.21 & 29.58 & 29.29 & 29.65 & 29.24 & 29.57 & 29.2 \\
\hline $\mathrm{H} 2 \mathrm{~b}$ & 29.8 & 29.85 & 29.81 & 29.87 & 29.79 & 29.81 & 29.83 & 29.83 \\
\hline H 3 & 25.78 & 25.99 & 25.97 & 26.01 & 25.87 & 26.05 & 25.98 & 25.98 \\
\hline H 5 & 29.52 & 29.52 & 29.56 & 29.64 & 29.48 & 29.52 & 29.53 & 29.55 \\
\hline H 7a & 29.29 & 29.39 & 29.27 & 29.31 & 29.29 & 29.34 & 29.31 & 29.28 \\
\hline $\mathrm{H} 7 \mathrm{~b}$ & 29.41 & 29.41 & 29.33 & 29.35 & 29.38 & 29.37 & 29.35 & 29.33 \\
\hline H 8a & 29.39 & 29.61 & 29.32 & 29.62 & 29.34 & 29.61 & 29.3 & 29.62 \\
\hline $\mathrm{H} 8 \mathrm{~b}$ & 29.9 & 29.75 & 29.99 & 29.65 & 29.89 & 29.72 & 30.04 & 29.67 \\
\hline H 10 & 29.99 & 30.02 & 30.03 & 29.95 & 30.03 & 30.05 & 30 & 29.94 \\
\hline H 11 & 29.41 & 29.4 & 29.41 & 29.45 & 29.49 & 29.43 & 29.45 & 29.41 \\
\hline H $12 a-c$ & 30.47 & 30.48 & 30.51 & 30.51 & 30.46 & 30.47 & 30.5 & 30.52 \\
\hline $\mathrm{H} 13 \mathrm{a}$ & 29.99 & 29.99 & 29.99 & 29.89 & 30 & 29.97 & 30.06 & 30.06 \\
\hline $\mathrm{H} 13 \mathrm{~b}$ & 30.08 & 30.1 & 30.31 & 30.29 & 30.31 & 30.27 & 30.19 & 30.17 \\
\hline H 14a & 30.02 & 30.13 & 29.87 & 29.95 & 29.82 & 29.9 & 29.82 & 29.81 \\
\hline $\mathrm{H} 14 \mathrm{~b}$ & 30.42 & 30.29 & 30.45 & 30.29 & 30.42 & 30.34 & 30.49 & 30.45 \\
\hline H 15 & 28.92 & 28.94 & 28.97 & 28.92 & 28.96 & 28.96 & 28.91 & 28.93 \\
\hline H 17a-c & 30.39 & 30.37 & 30.41 & 30.4 & 30.39 & 30.38 & 30.4 & 30.4 \\
\hline H 18a-c & 30.41 & 30.4 & 30.39 & 30.38 & 30.4 & 30.4 & 30.41 & 30.4 \\
\hline H 19a-c & 30.59 & 30.56 & 30.56 & 30.57 & 30.58 & 30.61 & 30.55 & 30.56 \\
\hline $\mathrm{H} 20 \mathrm{a}$ & 26.43 & 26.42 & 26.39 & 26.38 & 26.36 & 26.38 & 26.4 & 26.41 \\
\hline $\mathrm{H} 20 \mathrm{~b}$ & 26.58 & 26.56 & 26.6 & 26.61 & 26.64 & 26.59 & 26.61 & 26.62 \\
\hline
\end{tabular}


Table S4. Boltzmann averaged GIAO isotropic magnetic shielding constants $(\sigma)$ of isomers 1-16 calculated at the PCM/mPW1PW91/6-31+G**//B3LYP/6-31G* level of theory for compound 3

\begin{tabular}{|c|c|c|c|c|c|c|c|c|}
\hline \multicolumn{9}{|c|}{ Isotropic shielding constants } \\
\hline Nuclei & Isomer 1 & Isomer 2 & Isomer 3 & Isomer 4 & Isomer 5 & Isomer 6 & Isomer 7 & Isomer 8 \\
\hline C 1 & 161.21 & 161.41 & 162.04 & 162.33 & 161.64 & 164.71 & 160.90 & 163.01 \\
\hline $\mathrm{C} 2$ & 167.66 & 166.90 & 164.02 & 166.53 & 166.51 & 169.39 & 160.61 & 167.16 \\
\hline $\mathrm{C} 3$ & 167.56 & 161.42 & 164.88 & 168.38 & 159.31 & 177.87 & 156.15 & 175.78 \\
\hline C 4 & 118.75 & 119.03 & 117.96 & 117.79 & 118.35 & 116.54 & 117.04 & 116.54 \\
\hline $\mathrm{C} 5$ & 161.07 & 160.88 & 156.96 & 157.38 & 160.46 & 160.25 & 153.97 & 155.78 \\
\hline C 6 & 41.00 & 40.22 & 42.09 & 42.38 & 41.29 & 41.31 & 41.18 & 41.70 \\
\hline C 7 & 165.47 & 165.59 & 166.11 & 166.63 & 164.59 & 164.24 & 165.11 & 164.77 \\
\hline C 8 & 162.81 & 162.14 & 162.65 & 162.95 & 163.95 & 159.63 & 165.67 & 162.15 \\
\hline C 9 & 153.61 & 152.33 & 153.82 & 154.45 & 152.99 & 156.13 & 151.33 & 158.13 \\
\hline C 10 & 159.37 & 161.63 & 162.93 & 159.00 & 166.69 & 157.63 & 166.75 & 157.56 \\
\hline C 11 & 153.48 & 156.76 & 159.95 & 158.73 & 154.17 & 153.43 & 150.77 & 152.84 \\
\hline C 12 & 178.70 & 180.61 & 179.99 & 180.64 & 177.72 & 178.05 & 177.26 & 178.02 \\
\hline C 13 & 166.18 & 166.76 & 166.63 & 167.31 & 166.54 & 165.21 & 164.78 & 165.56 \\
\hline C 14 & 170.02 & 174.10 & 173.44 & 173.67 & 167.57 & 167.63 & 168.87 & 167.56 \\
\hline C 15 & 119.44 & 118.67 & 118.27 & 119.41 & 119.54 & 119.73 & 119.12 & 119.75 \\
\hline C 16 & 122.69 & 122.49 & 122.81 & 122.94 & 121.88 & 123.08 & 123.30 & 123.13 \\
\hline C 17 & 169.81 & 171.78 & 169.36 & 169.62 & 169.24 & 169.30 & 168.93 & 169.32 \\
\hline C 18 & 170.93 & 169.58 & 172.62 & 172.53 & 172.43 & 172.52 & 172.20 & 172.67 \\
\hline C 19 & 176.83 & 177.04 & 175.79 & 176.13 & 176.95 & 177.16 & 176.41 & 174.97 \\
\hline C 20 & 97.09 & 96.80 & 96.78 & 96.99 & 96.44 & 97.14 & 96.74 & 97.60 \\
\hline H 1 & 29.65 & 29.64 & 29.55 & 29.64 & 29.68 & 29.44 & 29.92 & 29.56 \\
\hline $\mathrm{H} 2 \mathrm{a}$ & 30.29 & 30.24 & 30.22 & 30.26 & 30.24 & 29.51 & 30.21 & 30.07 \\
\hline $\mathrm{H} 2 \mathrm{~b}$ & 30.42 & 30.38 & 30.49 & 30.39 & 30.38 & 30.77 & 30.35 & 30.34 \\
\hline H 3a & 30.09 & 29.93 & 29.95 & 29.44 & 29.92 & 29.79 & 29.85 & 29.70 \\
\hline $\mathrm{H} 3 \mathrm{~b}$ & 30.18 & 30.31 & 30.60 & 30.56 & 30.34 & 30.57 & 30.29 & 30.58 \\
\hline H 5 & 29.53 & 29.67 & 29.85 & 30.25 & 29.46 & 29.85 & 30.12 & 30.07 \\
\hline $\mathrm{H} 7 \mathrm{a}$ & 29.37 & 29.30 & 29.39 & 29.35 & 29.29 & 29.31 & 29.36 & 29.44 \\
\hline $\mathrm{H} 7 \mathrm{~b}$ & 29.41 & 29.40 & 29.54 & 29.41 & 29.31 & 29.37 & 29.47 & 29.47 \\
\hline $\mathrm{H} 8 \mathrm{a}$ & 29.65 & 29.75 & 29.61 & 29.63 & 29.49 & 29.71 & 29.42 & 29.63 \\
\hline $\mathrm{H} 8 \mathrm{~b}$ & 29.75 & 29.49 & 29.75 & 29.75 & 29.99 & 29.80 & 29.70 & 29.74 \\
\hline H 10 & 30.25 & 30.29 & 29.69 & 30.14 & 30.53 & 30.33 & 30.45 & 30.30 \\
\hline H 11 & 30.01 & 30.14 & 29.70 & 30.12 & 30.09 & 29.75 & 29.89 & 29.81 \\
\hline H 12a-c & 30.63 & 30.52 & 30.57 & 30.44 & 30.75 & 30.64 & 30.59 & 30.65 \\
\hline $\mathrm{H} 13 \mathrm{a}$ & 29.93 & 29.63 & 29.47 & 29.62 & 30.10 & 30.12 & 29.90 & 30.12 \\
\hline $\mathrm{H} 13 \mathrm{~b}$ & 30.12 & 30.09 & 30.07 & 30.16 & 30.12 & 30.14 & 30.17 & 30.15 \\
\hline H 14a & 30.03 & 29.97 & 29.98 & 29.90 & 30.17 & 30.12 & 30.10 & 30.10 \\
\hline $\mathrm{H} 14 \mathrm{~b}$ & 30.18 & 30.35 & 30.37 & 30.38 & 30.19 & 30.13 & 30.14 & 30.12 \\
\hline H 15 & 28.11 & 27.97 & 27.88 & 28.07 & 27.97 & 28.19 & 28.02 & 28.16 \\
\hline H 17a-c & 30.45 & 30.52 & 30.45 & 30.45 & 30.40 & 30.45 & 30.43 & 30.42 \\
\hline H 18a-c & 30.50 & 30.42 & 30.51 & 30.48 & 30.54 & 30.51 & 30.54 & 30.49 \\
\hline H 19a-c & 30.66 & 30.64 & 30.59 & 30.53 & 30.62 & 30.73 & 30.55 & 30.52 \\
\hline $\mathrm{H} 20 \mathrm{a}$ & 26.36 & 26.32 & 26.32 & 26.40 & 26.32 & 26.45 & 26.32 & 26.46 \\
\hline $\mathrm{H} 20 \mathrm{~b}$ & 26.61 & 26.68 & 26.55 & 26.58 & 26.45 & 26.73 & 26.49 & 26.69 \\
\hline
\end{tabular}




\begin{tabular}{|c|c|c|c|c|c|c|c|c|}
\hline \multicolumn{9}{|c|}{ Isotropic shielding constants } \\
\hline Nuclei & Isomer 9 & Isomer 10 & Isomer 11 & Isomer 12 & Isomer 13 & Isomer 14 & Isomer 15 & Isomer 16 \\
\hline C 1 & 160.35 & 164.47 & 162.30 & 164.20 & 162.43 & 163.65 & 162.84 & 163.42 \\
\hline $\mathrm{C} 2$ & 167.98 & 168.94 & 169.41 & 167.03 & 167.17 & 168.64 & 165.64 & 166.23 \\
\hline C 3 & 171.83 & 163.59 & 178.20 & 162.66 & 166.74 & 164.52 & 169.13 & 163.22 \\
\hline $\mathrm{C} 4$ & 118.93 & 117.52 & 118.08 & 117.75 & 118.89 & 118.75 & 118.32 & 119.11 \\
\hline C 5 & 162.22 & 158.61 & 159.63 & 154.05 & 162.00 & 160.77 & 158.30 & 155.79 \\
\hline C 6 & 41.34 & 42.09 & 40.62 & 42.81 & 39.53 & 40.41 & 40.69 & 41.60 \\
\hline C 7 & 165.43 & 166.07 & 164.53 & 165.88 & 166.01 & 165.54 & 165.41 & 166.44 \\
\hline C 8 & 163.81 & 159.74 & 161.72 & 162.42 & 161.11 & 160.35 & 162.91 & 162.54 \\
\hline$C 9$ & 153.64 & 156.11 & 154.04 & 157.69 & 154.04 & 153.69 & 154.59 & 154.95 \\
\hline C 10 & 156.26 & 168.26 & 157.10 & 167.75 & 160.31 & 164.17 & 161.47 & 164.94 \\
\hline C 11 & 152.44 & 151.87 & 151.03 & 152.37 & 159.05 & 159.49 & 160.12 & 158.99 \\
\hline C 12 & 178.02 & 177.02 & 177.65 & 177.22 & 179.79 & 180.39 & 179.43 & 180.22 \\
\hline C 13 & 166.24 & 165.00 & 166.09 & 165.90 & 166.90 & 167.60 & 166.57 & 167.71 \\
\hline C 14 & 168.47 & 167.27 & 167.88 & 167.33 & 173.96 & 174.27 & 173.64 & 174.47 \\
\hline C 15 & 119.87 & 119.58 & 119.97 & 119.73 & 119.20 & 119.14 & 119.24 & 119.70 \\
\hline C 16 & 122.71 & 123.31 & 122.25 & 123.20 & 122.47 & 122.28 & 122.66 & 122.79 \\
\hline C 17 & 172.00 & 172.60 & 172.11 & 172.59 & 172.41 & 172.60 & 172.46 & 172.75 \\
\hline C 18 & 168.14 & 169.62 & 168.66 & 169.54 & 169.16 & 169.57 & 169.43 & 169.70 \\
\hline C 19 & 176.79 & 177.08 & 177.44 & 173.94 & 177.09 & 177.75 & 176.10 & 175.33 \\
\hline C 20 & 97.36 & 96.95 & 96.75 & 96.74 & 97.56 & 97.25 & 97.41 & 97.32 \\
\hline H 1 & 29.69 & 29.49 & 29.57 & 29.56 & 29.62 & 29.57 & 29.52 & 29.50 \\
\hline $\mathrm{H} 2 \mathrm{a}$ & 30.34 & 29.38 & 30.07 & 29.95 & 30.16 & 29.71 & 30.00 & 30.08 \\
\hline $\mathrm{H} 2 \mathrm{~b}$ & 30.60 & 30.69 & 30.51 & 30.14 & 30.53 & 30.70 & 30.51 & 30.28 \\
\hline H За & 29.70 & 29.75 & 29.93 & 29.82 & 29.74 & 30.16 & 29.90 & 30.08 \\
\hline $\mathrm{H} 3 \mathrm{~b}$ & 30.54 & 30.49 & 30.35 & 30.42 & 30.19 & 30.31 & 30.02 & 30.41 \\
\hline H 5 & 29.41 & 29.90 & 29.39 & 30.05 & 29.55 & 30.17 & 29.70 & 30.33 \\
\hline H 7a & 29.36 & 29.35 & 29.37 & 29.31 & 29.38 & 29.27 & 29.41 & 29.39 \\
\hline $\mathrm{H} 7 \mathrm{~b}$ & 29.46 & 29.40 & 29.43 & 29.40 & 29.40 & 29.32 & 29.48 & 29.53 \\
\hline H 8a & 29.56 & 29.84 & 29.84 & 29.74 & 29.65 & 29.65 & 29.72 & 29.61 \\
\hline $\mathrm{H} 8 \mathrm{~b}$ & 29.88 & 29.90 & 29.73 & 29.82 & 29.90 & 29.77 & 29.76 & 29.83 \\
\hline H 10 & 30.31 & 29.72 & 30.18 & 29.84 & 30.00 & 29.55 & 29.97 & 29.52 \\
\hline H 11 & 29.97 & 30.04 & 29.99 & 30.09 & 29.88 & 29.92 & 29.80 & 29.88 \\
\hline H 12a-c & 30.69 & 30.61 & 30.65 & 30.67 & 30.39 & 30.61 & 30.38 & 30.64 \\
\hline $\mathrm{H} 13 \mathrm{a}$ & 30.03 & 30.06 & 30.09 & 29.99 & 29.57 & 29.44 & 29.59 & 29.45 \\
\hline $\mathrm{H} 13 \mathrm{~b}$ & 30.19 & 30.07 & 30.16 & 30.04 & 30.15 & 30.13 & 30.14 & 30.11 \\
\hline H 14a & 30.02 & 30.09 & 30.07 & 30.05 & 29.88 & 29.96 & 29.90 & 29.93 \\
\hline $\mathrm{H} 14 \mathrm{~b}$ & 30.12 & 30.10 & 30.14 & 30.07 & 30.42 & 30.38 & 30.43 & 30.36 \\
\hline H 15 & 28.24 & 27.89 & 28.14 & 27.92 & 28.10 & 27.84 & 28.10 & 27.93 \\
\hline H 17a-c & 30.55 & 30.49 & 30.50 & 30.52 & 30.52 & 30.47 & 30.50 & 30.49 \\
\hline H 18a-c & 30.42 & 30.41 & 30.43 & 30.42 & 30.46 & 30.43 & 30.47 & 30.44 \\
\hline H 19a-c & 30.67 & 30.75 & 30.57 & 30.62 & 30.68 & 30.72 & 30.55 & 30.56 \\
\hline $\mathrm{H} 20 \mathrm{a}$ & 26.39 & 26.39 & 26.32 & 26.40 & 26.34 & 26.49 & 26.35 & 26.41 \\
\hline $\mathrm{H} 20 \mathrm{~b}$ & 26.54 & 26.63 & 26.52 & 26.59 & 26.61 & 26.69 & 26.60 & 26.51 \\
\hline
\end{tabular}


Table S5. Boltzmann averaged GIAO isotropic magnetic shielding constants $(\sigma)$ of isomers 1-32 calculated at the PCM/mPW1PW91/6-31+G**//B3LYP/6-31G* level of theory for compound 4

\begin{tabular}{|c|c|c|c|c|c|c|c|c|}
\hline \multicolumn{9}{|c|}{ Isotropic shielding constants } \\
\hline Nuclei & Isomer 1 & Isomer 2 & Isomer 3 & Isomer 4 & Isomer 5 & Isomer 6 & Isomer 7 & Isomer 8 \\
\hline C 1 & 160.93 & 161.73 & 163.27 & 163.65 & 163.91 & 161.43 & 162.48 & 163.16 \\
\hline $\mathrm{C} 2$ & 166.61 & 167.77 & 168.17 & 168.59 & 167.01 & 168.30 & 166.53 & 166.41 \\
\hline $\mathrm{C} 3$ & 158.69 & 166.53 & 166.69 & 170.60 & 163.38 & 163.23 & 168.57 & 169.26 \\
\hline C 4 & 118.97 & 118.96 & 118.93 & 117.79 & 119.78 & 120.71 & 117.87 & 118.14 \\
\hline $\mathrm{C} 5$ & 161.94 & 162.06 & 162.79 & 166.04 & 160.57 & 157.44 & 161.67 & 161.64 \\
\hline C 6 & 110.23 & 110.22 & 110.37 & 110.64 & 108.96 & 110.72 & 109.31 & 110.53 \\
\hline C 7 & 160.67 & 159.93 & 159.73 & 160.22 & 159.71 & 161.26 & 159.12 & 159.75 \\
\hline C 8 & 164.18 & 163.62 & 161.62 & 161.29 & 162.41 & 162.65 & 162.52 & 164.48 \\
\hline C 9 & 157.06 & 158.35 & 157.43 & 158.17 & 156.58 & 157.34 & 158.75 & 160.85 \\
\hline C 10 & 171.37 & 166.18 & 166.60 & 164.06 & 166.71 & 168.13 & 162.86 & 163.54 \\
\hline C 11 & 153.61 & 153.14 & 157.56 & 158.26 & 155.51 & 155.36 & 158.76 & 157.89 \\
\hline C 12 & 177.37 & 177.57 & 180.62 & 181.19 & 180.45 & 180.59 & 179.88 & 180.77 \\
\hline C 13 & 165.97 & 166.39 & 167.42 & 167.59 & 167.01 & 167.44 & 166.87 & 167.52 \\
\hline C 14 & 168.62 & 169.31 & 174.23 & 174.00 & 174.02 & 175.18 & 173.83 & 173.79 \\
\hline C 15 & 119.38 & 119.95 & 119.85 & 119.87 & 119.56 & 120.06 & 119.68 & 119.80 \\
\hline C 16 & 122.64 & 122.80 & 122.61 & 122.71 & 122.25 & 122.46 & 122.76 & 122.77 \\
\hline C 17 & 169.59 & 170.58 & 172.04 & 169.39 & 169.71 & 167.80 & 169.50 & 169.23 \\
\hline C 18 & 172.06 & 169.94 & 169.34 & 172.55 & 172.26 & 171.68 & 172.66 & 172.64 \\
\hline C 19 & 176.35 & 176.84 & 177.24 & 177.00 & 177.37 & 177.52 & 175.43 & 176.08 \\
\hline C 20 & 173.80 & 174.38 & 173.54 & 172.85 & 174.64 & 173.00 & 174.08 & 172.73 \\
\hline $\mathrm{COCH}_{3}$ & 145.29 & 145.44 & 145.56 & 144.96 & 146.38 & 144.98 & 146.27 & 144.89 \\
\hline H 1 & 29.82 & 29.78 & 29.62 & 29.66 & 29.47 & 29.63 & 29.59 & 29.74 \\
\hline $\mathrm{H} 2 \mathrm{a}$ & 30.35 & 30.22 & 30.13 & 29.76 & 30.00 & 30.00 & 30.25 & 30.19 \\
\hline $\mathrm{H} 2 \mathrm{~b}$ & 30.47 & 30.52 & 30.37 & 30.80 & 30.38 & 30.48 & 30.37 & 30.42 \\
\hline H $3 a$ & 30.00 & 29.98 & 30.09 & 29.46 & 30.21 & 30.25 & 29.42 & 29.36 \\
\hline $\mathrm{H} 3 \mathrm{~b}$ & 30.34 & 30.35 & 30.35 & 30.79 & 30.35 & 30.37 & 30.64 & 30.77 \\
\hline H 5 & 30.00 & 30.00 & 30.38 & 30.62 & 29.85 & 30.12 & 30.44 & 30.83 \\
\hline H 7a & 30.03 & 30.01 & 30.08 & 29.97 & 29.93 & 30.02 & 30.03 & 30.06 \\
\hline $\mathrm{H} 7 \mathrm{~b}$ & 30.10 & 30.06 & 30.09 & 30.11 & 30.29 & 30.14 & 30.13 & 30.10 \\
\hline $\mathrm{H} 8 \mathrm{a}$ & 29.55 & 29.75 & 29.76 & 29.81 & 29.70 & 29.68 & 29.52 & 29.84 \\
\hline $\mathrm{H} 8 \mathrm{~b}$ & 29.97 & 29.84 & 29.85 & 29.96 & 29.91 & 29.87 & 29.93 & 29.90 \\
\hline H 10 & 30.51 & 30.33 & 30.13 & 30.20 & 30.69 & 30.23 & 30.53 & 30.20 \\
\hline H 11 & 30.09 & 30.02 & 30.09 & 30.14 & 30.14 & 30.12 & 30.15 & 30.12 \\
\hline H 12a-c & 30.70 & 30.65 & 30.48 & 30.26 & 30.58 & 30.59 & 30.38 & 30.29 \\
\hline $\mathrm{H} 13 \mathrm{a}$ & 29.97 & 29.94 & 29.49 & 29.60 & 29.48 & 29.37 & 29.64 & 29.55 \\
\hline $\mathrm{H} 13 \mathrm{~b}$ & 30.09 & 30.05 & 30.11 & 30.15 & 30.16 & 30.15 & 30.17 & 30.20 \\
\hline H 14a & 30.14 & 30.05 & 29.93 & 29.85 & 29.97 & 29.81 & 29.91 & 29.88 \\
\hline $\mathrm{H} 14 \mathrm{~b}$ & 30.15 & 30.14 & 30.34 & 30.38 & 30.42 & 30.44 & 30.41 & 30.42 \\
\hline H 15 & 27.73 & 27.88 & 27.85 & 28.08 & 27.88 & 27.46 & 28.10 & 28.12 \\
\hline H 17a-c & 30.41 & 30.50 & 30.51 & 30.47 & 30.43 & 30.34 & 30.45 & 30.44 \\
\hline H 18a-c & 30.53 & 30.46 & 30.43 & 30.47 & 30.52 & 30.56 & 30.49 & 30.48 \\
\hline H 19a-c & 30.62 & 30.63 & 30.69 & 30.78 & 30.54 & 30.59 & 30.55 & 30.62 \\
\hline H 20a-c & 30.27 & 30.29 & 30.32 & 30.34 & 30.24 & 30.34 & 30.24 & 30.26 \\
\hline $\mathrm{HOCH}_{3}$ & 28.25 & 28.23 & 28.25 & 28.20 & 28.30 & 28.28 & 28.23 & 28.26 \\
\hline
\end{tabular}




\begin{tabular}{|c|c|c|c|c|c|c|c|c|}
\hline \multicolumn{9}{|c|}{ Isotropic shielding constants } \\
\hline Nuclei & Isomer 9 & Isomer 10 & Isomer 11 & Isomer 12 & Isomer 13 & Isomer 14 & Isomer 15 & Isomer 16 \\
\hline C 1 & 161.69 & 160.50 & 164.50 & 164.60 & 162.12 & 162.02 & 162.92 & 162.83 \\
\hline$C 2$ & 166.55 & 166.56 & 169.41 & 168.96 & 167.43 & 168.43 & 166.79 & 167.35 \\
\hline C 3 & 158.27 & 158.76 & 178.41 & 177.96 & 163.83 & 163.95 & 176.13 & 176.35 \\
\hline C 4 & 118.71 & 118.96 & 116.23 & 115.81 & 117.96 & 119.05 & 116.22 & 116.47 \\
\hline C 5 & 163.43 & 161.28 & 164.47 & 158.18 & 162.11 & 158.84 & 158.68 & 154.54 \\
\hline C 6 & 109.58 & 110.55 & 108.76 & 110.26 & 108.26 & 110.71 & 108.92 & 110.71 \\
\hline$C 7$ & 159.97 & 161.02 & 156.88 & 163.54 & 159.31 & 160.90 & 158.44 & 163.74 \\
\hline C 8 & 164.01 & 164.36 & 160.36 & 160.62 & 161.92 & 163.55 & 163.30 & 163.00 \\
\hline C 9 & 155.32 & 157.90 & 160.30 & 160.44 & 156.61 & 158.10 & 160.89 & 163.60 \\
\hline C 10 & 170.48 & 172.22 & 162.14 & 162.36 & 172.15 & 171.89 & 161.77 & 162.29 \\
\hline C 11 & 153.61 & 153.46 & 153.86 & 153.08 & 153.87 & 153.25 & 153.48 & 153.27 \\
\hline C 12 & 177.29 & 177.14 & 177.29 & 177.56 & 177.80 & 176.27 & 177.33 & 178.09 \\
\hline C 13 & 165.71 & 165.96 & 165.34 & 165.25 & 166.61 & 166.76 & 165.50 & 165.29 \\
\hline C 14 & 168.66 & 168.09 & 167.58 & 167.35 & 167.33 & 168.80 & 167.63 & 167.71 \\
\hline C 15 & 118.65 & 119.67 & 118.93 & 120.12 & 120.08 & 120.13 & 119.22 & 119.49 \\
\hline C 16 & 123.15 & 122.44 & 123.18 & 122.81 & 122.10 & 123.07 & 123.14 & 123.03 \\
\hline C 17 & 170.07 & 169.41 & 169.31 & 169.04 & 170.16 & 167.97 & 169.34 & 169.57 \\
\hline C 18 & 172.01 & 172.06 & 172.52 & 172.23 & 172.51 & 171.11 & 172.65 & 172.87 \\
\hline C 19 & 176.12 & 176.42 & 176.71 & 176.13 & 177.60 & 177.87 & 174.82 & 174.96 \\
\hline C 20 & 174.97 & 173.23 & 177.80 & 173.89 & 174.31 & 173.20 & 174.72 & 173.86 \\
\hline $\mathrm{COCH}_{3}$ & 146.39 & 144.75 & 146.91 & 145.51 & 145.70 & 144.85 & 145.69 & 145.64 \\
\hline H 1 & 29.76 & 29.85 & 29.54 & 29.59 & 29.46 & 29.73 & 29.49 & 29.64 \\
\hline $\mathrm{H} 2 \mathrm{a}$ & 30.28 & 30.38 & 29.52 & 29.60 & 29.89 & 29.97 & 30.05 & 30.15 \\
\hline $\mathrm{H} 2 \mathrm{~b}$ & 30.43 & 30.51 & 30.80 & 30.81 & 30.47 & 30.48 & 30.39 & 30.40 \\
\hline H $3 a$ & 29.95 & 29.97 & 29.71 & 29.80 & 29.71 & 29.77 & 29.63 & 29.66 \\
\hline $\mathrm{H} 3 \mathrm{~b}$ & 30.35 & 30.36 & 30.49 & 30.59 & 30.63 & 30.59 & 30.54 & 30.56 \\
\hline H 5 & 29.80 & 30.08 & 30.03 & 30.53 & 29.79 & 30.08 & 30.29 & 30.68 \\
\hline $\mathrm{H} 7 \mathrm{a}$ & 29.98 & 30.05 & 30.11 & 30.04 & 29.87 & 29.91 & 30.06 & 30.03 \\
\hline $\mathrm{H} 7 \mathrm{~b}$ & 30.36 & 29.98 & 30.28 & 30.14 & 30.26 & 30.00 & 30.33 & 30.17 \\
\hline $\mathrm{H} 8 \mathrm{a}$ & 29.45 & 29.59 & 29.58 & 29.71 & 29.69 & 29.83 & 29.46 & 29.78 \\
\hline $\mathrm{H} 8 \mathrm{~b}$ & 30.13 & 29.90 & 29.80 & 29.90 & 29.84 & 29.97 & 29.80 & 29.80 \\
\hline H 10 & 30.84 & 30.37 & 30.82 & 30.33 & 30.75 & 30.26 & 30.87 & 30.32 \\
\hline H 11 & 30.13 & 30.07 & 29.80 & 29.67 & 30.13 & 30.09 & 29.84 & 29.71 \\
\hline H 12a-c & 30.69 & 30.70 & 30.66 & 30.62 & 30.71 & 30.64 & 30.66 & 30.62 \\
\hline H 13a & 30.07 & 29.96 & 30.15 & 30.08 & 30.09 & 29.96 & 30.16 & 30.09 \\
\hline $\mathrm{H} 13 \mathrm{~b}$ & 30.13 & 30.09 & 30.15 & 30.15 & 30.12 & 30.06 & 30.16 & 30.20 \\
\hline H 14a & 30.11 & 30.13 & 30.11 & 30.09 & 30.10 & 30.05 & 30.13 & 30.10 \\
\hline $\mathrm{H} 14 \mathrm{~b}$ & 30.14 & 30.17 & 30.16 & 30.09 & 30.19 & 30.10 & 30.16 & 30.11 \\
\hline H 15 & 28.01 & 27.60 & 28.18 & 28.17 & 27.86 & 27.57 & 28.16 & 28.26 \\
\hline H 17a-c & 30.40 & 30.42 & 30.45 & 30.46 & 30.43 & 30.37 & 30.43 & 30.45 \\
\hline H 18a-c & 30.51 & 30.55 & 30.53 & 30.50 & 30.55 & 30.59 & 30.52 & 30.51 \\
\hline H 19a-c & 30.60 & 30.63 & 30.74 & 30.74 & 30.51 & 30.61 & 30.51 & 30.61 \\
\hline H 20a-c & 30.23 & 30.28 & 30.32 & 30.33 & 30.14 & 30.28 & 30.32 & 30.28 \\
\hline $\mathrm{HOCH}_{3}$ & 28.23 & 28.25 & 28.33 & 28.33 & 28.25 & 28.19 & 28.30 & 28.36 \\
\hline
\end{tabular}




\begin{tabular}{|c|c|c|c|c|c|c|c|c|}
\hline \multicolumn{9}{|c|}{ Isotropic shielding constants } \\
\hline Nuclei & Isomer 17 & Isomer 18 & Isomer 19 & Isomer 20 & Isomer $\mathbf{2 1}$ & Isomer 22 & Isomer 23 & Isomer 24 \\
\hline C 1 & 161.14 & 160.96 & 164.56 & 164.74 & 161.23 & 161.72 & 163.43 & 163.51 \\
\hline $\mathrm{C} 2$ & 167.73 & 167.30 & 169.15 & 168.95 & 168.72 & 169.04 & 166.70 & 166.17 \\
\hline C 3 & 171.99 & 171.80 & 164.59 & 164.62 & 177.62 & 178.29 & 161.91 & 162.63 \\
\hline $\mathrm{C} 4$ & 118.28 & 119.35 & 116.72 & 117.59 & 118.34 & 118.47 & 117.77 & 117.72 \\
\hline $\mathrm{C} 5$ & 167.05 & 159.97 & 164.77 & 158.23 & 163.56 & 161.81 & 160.27 & 153.95 \\
\hline C 6 & 109.34 & 111.30 & 109.44 & 109.93 & 109.35 & 110.71 & 109.61 & 110.69 \\
\hline C 7 & 157.14 & 161.44 & 157.00 & 163.36 & 158.09 & 158.94 & 157.93 & 162.97 \\
\hline C 8 & 164.57 & 163.58 & 159.92 & 161.04 & 161.42 & 163.60 & 163.07 & 163.00 \\
\hline$C 9$ & 157.48 & 158.21 & 159.04 & 161.79 & 157.69 & 159.19 & 159.80 & 162.99 \\
\hline C 10 & 160.93 & 161.93 & 171.12 & 170.90 & 160.32 & 162.70 & 169.65 & 170.56 \\
\hline C 11 & 152.33 & 152.57 & 150.95 & 151.70 & 150.33 & 151.29 & 151.46 & 150.90 \\
\hline C 12 & 177.65 & 177.21 & 176.48 & 176.44 & 176.64 & 176.78 & 176.61 & 177.54 \\
\hline C 13 & 166.01 & 166.01 & 164.79 & 164.98 & 166.23 & 166.19 & 165.10 & 165.57 \\
\hline C 14 & 167.51 & 168.07 & 167.77 & 166.92 & 167.06 & 167.50 & 167.25 & 167.39 \\
\hline C 15 & 119.96 & 119.93 & 119.26 & 119.24 & 119.50 & 119.84 & 119.61 & 119.48 \\
\hline C 16 & 123.19 & 122.19 & 122.55 & 122.81 & 123.05 & 122.79 & 122.63 & 122.79 \\
\hline C 17 & 172.20 & 172.25 & 172.46 & 172.53 & 172.08 & 171.97 & 172.54 & 172.51 \\
\hline C 18 & 168.92 & 168.81 & 169.26 & 169.66 & 169.08 & 168.88 & 169.33 & 169.31 \\
\hline C 19 & 175.90 & 176.71 & 177.22 & 176.44 & 177.15 & 177.09 & 173.90 & 174.42 \\
\hline C 20 & 176.63 & 173.85 & 176.17 & 173.39 & 174.51 & 172.70 & 174.18 & 173.76 \\
\hline $\mathrm{COCH}_{3}$ & 146.55 & 145.59 & 147.58 & 145.62 & 145.77 & 144.61 & 146.49 & 145.92 \\
\hline H 1 & 29.74 & 29.87 & 29.61 & 29.66 & 29.53 & 29.65 & 29.50 & 29.61 \\
\hline $\mathrm{H} 2 \mathrm{a}$ & 30.35 & 30.36 & 29.43 & 29.41 & 30.07 & 30.12 & 29.96 & 29.97 \\
\hline $\mathrm{H} 2 \mathrm{~b}$ & 30.64 & 30.68 & 30.73 & 30.76 & 30.46 & 30.52 & 30.22 & 30.29 \\
\hline H $3 a$ & 29.68 & 29.71 & 29.63 & 29.82 & 29.93 & 29.97 & 29.81 & 29.74 \\
\hline $\mathrm{H} 3 \mathrm{~b}$ & 30.54 & 30.59 & 30.41 & 30.44 & 30.33 & 30.34 & 30.42 & 30.41 \\
\hline H 5 & 29.58 & 29.98 & 30.04 & 30.47 & 29.69 & 30.03 & 30.29 & 30.51 \\
\hline H 7a & 30.11 & 30.00 & 30.13 & 30.06 & 30.01 & 30.09 & 30.16 & 30.04 \\
\hline $\mathrm{H} 7 \mathrm{~b}$ & 30.18 & 30.06 & 30.26 & 30.07 & 30.15 & 30.13 & 30.23 & 30.11 \\
\hline $\mathrm{H} 8 \mathrm{a}$ & 29.41 & 29.62 & 29.66 & 29.81 & 29.71 & 29.71 & 29.61 & 29.77 \\
\hline $\mathrm{H} 8 \mathrm{~b}$ & 30.11 & 29.87 & 29.82 & 29.88 & 29.83 & 29.96 & 29.96 & 29.78 \\
\hline H 10 & 30.65 & 30.27 & 30.16 & 29.65 & 30.58 & 30.24 & 30.13 & 29.67 \\
\hline H 11 & 29.94 & 29.89 & 30.06 & 30.08 & 29.94 & 29.89 & 30.06 & 30.17 \\
\hline H 12a-c & 30.69 & 30.66 & 30.60 & 30.59 & 30.66 & 30.64 & 30.62 & 30.56 \\
\hline $\mathrm{H} 13 \mathrm{a}$ & 30.07 & 30.05 & 29.94 & 29.83 & 30.05 & 30.04 & 29.89 & 29.82 \\
\hline $\mathrm{H} 13 \mathrm{~b}$ & 30.16 & 30.17 & 30.07 & 30.10 & 30.21 & 30.15 & 30.07 & 30.10 \\
\hline H 14a & 30.05 & 30.06 & 30.02 & 30.04 & 30.04 & 30.04 & 30.05 & 30.04 \\
\hline $\mathrm{H} 14 \mathrm{~b}$ & 30.12 & 30.17 & 30.12 & 30.12 & 30.16 & 30.13 & 30.08 & 30.17 \\
\hline H 15 & 28.22 & 28.24 & 27.88 & 27.78 & 28.13 & 28.17 & 27.84 & 27.81 \\
\hline H 17a-c & 30.50 & 30.53 & 30.48 & 30.50 & 30.50 & 30.53 & 30.46 & 30.50 \\
\hline H 18a-c & 30.45 & 30.47 & 30.43 & 30.42 & 30.44 & 30.46 & 30.39 & 30.44 \\
\hline H 19a-c & 30.61 & 30.61 & 30.75 & 30.73 & 30.55 & 30.59 & 30.58 & 30.60 \\
\hline H 20a-c & 30.31 & 30.32 & 30.30 & 30.34 & 30.31 & 30.31 & 30.34 & 30.36 \\
\hline $\mathrm{HOCH}_{3}$ & 28.18 & 28.32 & 28.33 & 28.33 & 28.25 & 28.14 & 28.27 & 28.36 \\
\hline
\end{tabular}




\begin{tabular}{|c|c|c|c|c|c|c|c|c|}
\hline \multicolumn{9}{|c|}{ Isotropic shielding constants } \\
\hline Nuclei & Isomer 25 & Isomer 26 & Isomer 27 & Isomer 28 & Isomer 29 & Isomer 30 & Isomer 31 & Isomer 32 \\
\hline C 1 & 160.98 & 162.49 & 163.91 & 164.09 & 161.63 & 162.46 & 162.15 & 161.10 \\
\hline$C 2$ & 167.63 & 166.43 & 168.21 & 168.09 & 166.56 & 166.11 & 166.11 & 165.70 \\
\hline C 3 & 164.48 & 167.23 & 164.85 & 165.26 & 169.65 & 168.88 & 162.91 & 163.09 \\
\hline C 4 & 120.57 & 119.98 & 118.85 & 119.04 & 119.52 & 118.75 & 118.95 & 119.12 \\
\hline C 5 & 166.99 & 163.00 & 164.49 & 163.49 & 162.78 & 161.91 & 160.33 & 158.88 \\
\hline C 6 & 109.17 & 110.00 & 109.51 & 111.31 & 109.39 & 110.73 & 109.53 & 111.43 \\
\hline C 7 & 157.66 & 159.51 & 158.15 & 158.70 & 158.30 & 158.65 & 158.36 & 159.61 \\
\hline C 8 & 162.63 & 161.98 & 160.00 & 161.56 & 161.93 & 163.81 & 162.31 & 164.58 \\
\hline C 9 & 158.87 & 158.52 & 156.30 & 156.93 & 157.66 & 157.89 & 157.62 & 157.83 \\
\hline C 10 & 163.78 & 164.95 & 167.86 & 169.87 & 164.81 & 166.07 & 167.71 & 169.75 \\
\hline C 11 & 156.29 & 159.59 & 159.03 & 158.22 & 158.72 & 158.97 & 158.73 & 157.85 \\
\hline C 12 & 179.86 & 179.97 & 180.39 & 180.37 & 179.45 & 179.64 & 180.25 & 180.25 \\
\hline C 13 & 167.03 & 166.99 & 166.86 & 167.88 & 167.45 & 166.75 & 166.94 & 167.74 \\
\hline C 14 & 173.89 & 173.77 & 173.67 & 174.04 & 173.53 & 173.48 & 174.73 & 174.23 \\
\hline C 15 & 119.68 & 119.29 & 119.77 & 119.93 & 119.56 & 119.38 & 119.22 & 119.02 \\
\hline C 16 & 122.90 & 122.50 & 122.65 & 122.77 & 122.57 & 122.51 & 122.12 & 122.55 \\
\hline C 17 & 172.41 & 172.61 & 172.46 & 172.41 & 172.39 & 172.34 & 172.46 & 172.63 \\
\hline C 18 & 168.80 & 169.21 & 169.28 & 169.28 & 169.30 & 168.71 & 169.19 & 169.34 \\
\hline C 19 & 176.09 & 177.88 & 177.37 & 177.59 & 176.26 & 176.47 & 175.04 & 175.80 \\
\hline C 20 & 176.48 & 173.35 & 175.91 & 172.87 & 174.91 & 173.07 & 175.02 & 172.57 \\
\hline $\mathrm{COCH}_{3}$ & 146.78 & 145.16 & 147.34 & 145.17 & 145.97 & 144.55 & 146.22 & 144.84 \\
\hline H 1 & 29.78 & 29.81 & 29.55 & 29.64 & 29.55 & 29.64 & 29.55 & 29.79 \\
\hline $\mathrm{H} 2 \mathrm{a}$ & 30.32 & 30.24 & 29.69 & 29.71 & 30.09 & 30.15 & 30.15 & 30.22 \\
\hline $\mathrm{H} 2 \mathrm{~b}$ & 30.64 & 30.63 & 30.69 & 30.75 & 30.54 & 30.56 & 30.30 & 30.40 \\
\hline H 3a & 29.11 & 29.87 & 30.14 & 30.17 & 29.85 & 29.96 & 30.08 & 30.03 \\
\hline $\mathrm{H} 3 \mathrm{~b}$ & 30.74 & 30.21 & 30.41 & 30.46 & 30.03 & 30.09 & 30.37 & 30.51 \\
\hline H 5 & 29.60 & 30.23 & 30.36 & 30.64 & 29.85 & 30.31 & 30.49 & 30.77 \\
\hline H 7a & 30.12 & 30.04 & 30.09 & 30.06 & 30.05 & 30.08 & 30.05 & 30.04 \\
\hline $\mathrm{H} 7 \mathrm{~b}$ & 30.19 & 30.07 & 30.24 & 30.12 & 30.26 & 30.08 & 30.20 & 30.11 \\
\hline H 8a & 29.44 & 29.74 & 29.60 & 29.77 & 29.62 & 29.75 & 29.52 & 29.78 \\
\hline $\mathrm{H} 8 \mathrm{~b}$ & 30.10 & 29.80 & 29.88 & 29.83 & 29.88 & 29.92 & 29.93 & 29.83 \\
\hline H 10 & 30.50 & 30.01 & 29.84 & 29.61 & 30.35 & 29.98 & 30.06 & 29.69 \\
\hline H 11 & 30.11 & 29.88 & 29.97 & 29.96 & 29.84 & 29.82 & 29.90 & 29.90 \\
\hline H 12a-c & 30.34 & 30.34 & 30.61 & 30.63 & 30.38 & 30.33 & 30.62 & 30.62 \\
\hline H 13a & 29.54 & 29.59 & 29.41 & 29.18 & 29.61 & 29.63 & 29.48 & 29.25 \\
\hline H 13b & 30.15 & 30.17 & 30.12 & 30.18 & 30.12 & 30.13 & 30.16 & 30.20 \\
\hline H 14a & 29.78 & 29.92 & 29.92 & 29.94 & 29.86 & 29.84 & 29.93 & 29.93 \\
\hline H 14b & 30.40 & 30.43 & 30.34 & 30.33 & 30.39 & 30.39 & 30.37 & 30.30 \\
\hline H 15 & 28.15 & 28.13 & 27.88 & 27.79 & 28.10 & 28.07 & 27.87 & 27.86 \\
\hline H 17a-c & 30.50 & 30.47 & 30.50 & 30.51 & 30.51 & 30.52 & 30.47 & 30.47 \\
\hline H 18a-c & 30.44 & 30.50 & 30.45 & 30.45 & 30.45 & 30.47 & 30.40 & 30.44 \\
\hline H 19a-c & 30.62 & 30.69 & 30.71 & 30.75 & 30.58 & 30.64 & 30.55 & 30.58 \\
\hline H 20a-c & 30.29 & 30.29 & 30.33 & 30.32 & 30.30 & 30.36 & 30.34 & 30.33 \\
\hline $\mathrm{H} \mathrm{OCH}_{3}$ & 28.18 & 28.26 & 28.28 & 28.22 & 28.26 & 28.17 & 28.24 & 28.23 \\
\hline
\end{tabular}


Table S6. Boltzmann averaged GIAO isotropic magnetic shielding constants $(\sigma)$ of isomers 1-8 calculated at the PCM/mPW1PW91/6-31+G**//B3LYP/6-31G* level of theory for compound 5

\begin{tabular}{|c|c|c|c|c|c|c|c|c|}
\hline \multicolumn{9}{|c|}{ Isotropic shielding constants } \\
\hline Nuclei & Isomer 1 & Isomer 2 & Isomer 3 & Isomer 4 & Isomer 5 & Isomer 6 & Isomer 7 & Isomer 8 \\
\hline C 1 & 165.06 & 166.41 & 164.67 & 166.19 & 165.02 & 166.21 & 164.91 & 165.97 \\
\hline$C 2$ & 162.90 & 161.03 & 162.93 & 161.33 & 162.64 & 161.50 & 162.76 & 160.99 \\
\hline C 3 & 82.92 & 81.18 & 83.47 & 80.23 & 83.85 & 79.82 & 83.32 & 80.67 \\
\hline C 4 & 51.37 & 50.87 & 51.04 & 52.43 & 51.34 & 51.99 & 51.21 & 50.17 \\
\hline C 5 & 152.72 & 154.37 & 152.61 & 154.82 & 152.53 & 154.83 & 152.26 & 154.24 \\
\hline C 6 & 42.25 & 43.09 & 41.95 & 42.26 & 42.80 & 41.58 & 42.74 & 42.50 \\
\hline C 7 & 164.97 & 165.15 & 165.15 & 164.51 & 164.54 & 165.08 & 165.04 & 165.30 \\
\hline C 8 & 161.80 & 164.87 & 162.18 & 164.40 & 162.10 & 164.21 & 162.50 & 165.34 \\
\hline C 9 & 151.61 & 151.24 & 152.00 & 152.25 & 151.55 & 152.17 & 151.17 & 151.44 \\
\hline C 10 & 167.18 & 165.95 & 166.73 & 165.78 & 168.04 & 166.92 & 167.10 & 165.94 \\
\hline C 11 & 109.33 & 109.25 & 109.09 & 108.54 & 109.29 & 109.21 & 109.14 & 108.90 \\
\hline C 12 & 166.94 & 167.33 & 167.08 & 168.12 & 167.58 & 168.70 & 166.77 & 169.52 \\
\hline C 13 & 157.19 & 156.92 & 157.18 & 157.34 & 156.46 & 156.36 & 157.63 & 157.33 \\
\hline C 14 & 167.05 & 167.15 & 166.94 & 167.42 & 167.15 & 166.97 & 167.01 & 167.40 \\
\hline C 15 & 110.00 & 109.92 & 109.79 & 110.50 & 109.23 & 108.94 & 109.96 & 111.31 \\
\hline C 16 & 123.84 & 123.57 & 124.66 & 123.41 & 124.92 & 123.90 & 124.11 & 122.99 \\
\hline C 17 & 171.46 & 171.56 & 171.88 & 172.33 & 167.52 & 168.39 & 167.71 & 168.29 \\
\hline C 18 & 167.63 & 167.84 & 167.75 & 168.88 & 171.81 & 172.22 & 171.59 & 171.65 \\
\hline C 19 & 176.97 & 176.87 & 177.12 & 176.49 & 177.16 & 176.67 & 176.67 & 176.69 \\
\hline C 20 & 95.73 & 96.58 & 96.68 & 96.71 & 96.30 & 96.98 & 96.03 & 96.82 \\
\hline H 1 & 29.52 & 29.66 & 29.53 & 29.68 & 29.56 & 29.70 & 29.52 & 29.70 \\
\hline $\mathrm{H} 2 \mathrm{a}$ & 29.21 & 29.47 & 29.19 & 29.48 & 29.26 & 29.52 & 29.23 & 29.55 \\
\hline $\mathrm{H} 2 \mathrm{~b}$ & 29.68 & 29.77 & 29.67 & 29.86 & 29.69 & 29.85 & 29.70 & 29.86 \\
\hline H 3 & 25.73 & 25.69 & 25.74 & 25.70 & 25.70 & 25.81 & 25.76 & 25.78 \\
\hline H 5 & 29.54 & 29.46 & 29.56 & 29.61 & 29.58 & 29.48 & 29.57 & 29.55 \\
\hline $\mathrm{H} 7 \mathrm{a}$ & 29.28 & 29.29 & 29.30 & 29.28 & 29.37 & 29.33 & 29.32 & 29.33 \\
\hline $\mathrm{H} 7 \mathrm{~b}$ & 29.34 & 29.33 & 29.33 & 29.31 & 29.44 & 29.38 & 29.36 & 29.33 \\
\hline H 8a & 29.60 & 29.31 & 29.54 & 29.34 & 29.67 & 29.44 & 29.56 & 29.31 \\
\hline $\mathrm{H} 8 \mathrm{~b}$ & 29.72 & 30.00 & 29.64 & 29.90 & 29.73 & 30.01 & 29.62 & 29.95 \\
\hline H 10 & 29.79 & 29.93 & 29.77 & 30.00 & 30.00 & 29.76 & 29.84 & 29.61 \\
\hline H 12a-c & 30.25 & 30.22 & 30.28 & 30.24 & 30.26 & 30.22 & 30.22 & 30.29 \\
\hline H $13 a$ & 29.28 & 29.31 & 29.46 & 29.39 & 29.54 & 29.31 & 29.20 & 29.62 \\
\hline $\mathrm{H} 13 \mathrm{~b}$ & 29.71 & 29.72 & 29.63 & 29.91 & 29.59 & 29.77 & 29.82 & 29.74 \\
\hline H 14a & 29.84 & 29.80 & 29.87 & 29.76 & 29.46 & 29.53 & 29.53 & 29.51 \\
\hline $\mathrm{H} 14 \mathrm{~b}$ & 29.58 & 29.57 & 29.48 & 29.62 & 29.87 & 29.80 & 29.90 & 29.58 \\
\hline H 15 & 27.53 & 27.57 & 27.69 & 27.68 & 27.61 & 27.53 & 27.49 & 27.49 \\
\hline H 17a-c & 30.55 & 30.55 & 30.54 & 30.54 & 30.38 & 30.41 & 30.38 & 30.41 \\
\hline H 18a-c & 30.39 & 30.39 & 30.40 & 30.44 & 30.54 & 30.50 & 30.59 & 30.57 \\
\hline H 19a-c & 30.60 & 30.52 & 30.57 & 30.57 & 30.58 & 30.59 & 30.58 & 30.56 \\
\hline H $20 \mathrm{a}$ & 26.34 & 26.39 & 26.41 & 26.42 & 26.42 & 26.43 & 26.40 & 26.36 \\
\hline $\mathrm{H} 20 \mathrm{~b}$ & 26.54 & 26.58 & 26.61 & 26.63 & 26.50 & 26.55 & 26.55 & 26.59 \\
\hline
\end{tabular}


Table S7. Boltzmann averaged GIAO isotropic magnetic shielding constants $(\sigma)$ of isomers 1-4 calculated at the PCM/mPW1PW91/6-31+G**//B3LYP/6-31G* level of theory for compound isoelisabethatriene (vacuum).

\begin{tabular}{|c|c|c|c|c|}
\hline Nuclei & Isomer 1 & Isomer 2 & Isomer 3 & Isomer 4 \\
\hline C 1 & 64.06 & 64.41 & 64.56 & 64.46 \\
\hline$C 2$ & 75.07 & 74.80 & 75.00 & 74.20 \\
\hline C 3 & 63.22 & 64.26 & 63.58 & 64.05 \\
\hline C 4 & 164.93 & 164.96 & 164.88 & 164.88 \\
\hline$C 5$ & 164.03 & 165.79 & 164.13 & 165.17 \\
\hline C 6 & 61.35 & 62.20 & 61.74 & 61.83 \\
\hline C 7 & 158.92 & 158.81 & 158.92 & 158.91 \\
\hline C 8 & 165.04 & 162.21 & 165.18 & 162.80 \\
\hline C 9 & 175.55 & 172.75 & 176.30 & 172.57 \\
\hline C 10 & 149.58 & 152.34 & 152.32 & 149.86 \\
\hline C 11 & 159.69 & 159.47 & 160.21 & 158.28 \\
\hline C 12 & 163.96 & 158.34 & 158.58 & 163.03 \\
\hline C 13 & 165.62 & 165.99 & 165.98 & 165.33 \\
\hline C 14 & 71.10 & 71.17 & 70.95 & 70.70 \\
\hline C 15 & 65.28 & 64.83 & 65.31 & 65.65 \\
\hline C 16 & 177.55 & 177.61 & 177.55 & 177.58 \\
\hline C 17 & 168.68 & 168.64 & 168.57 & 168.61 \\
\hline C 18 & 176.96 & 181.11 & 181.30 & 176.95 \\
\hline C 19 & 174.33 & 175.88 & 174.13 & 175.89 \\
\hline C 20 & 170.88 & 171.26 & 171.03 & 171.43 \\
\hline $\mathrm{H} 2$ & 25.53 & 25.50 & 25.58 & 25.54 \\
\hline $\mathrm{H} 4 \mathrm{a}$ & 29.69 & 29.41 & 29.76 & 29.43 \\
\hline $\mathrm{H} 4 \mathrm{~b}$ & 29.41 & 29.72 & 29.37 & 29.74 \\
\hline H $5 a$ & 29.45 & 29.54 & 29.34 & 29.60 \\
\hline $\mathrm{H} 5 \mathrm{~b}$ & 29.54 & 29.33 & 29.62 & 29.31 \\
\hline H 7 & 29.68 & 29.26 & 29.71 & 29.29 \\
\hline H $8 a$ & 30.20 & 30.41 & 30.16 & 30.43 \\
\hline $\mathrm{H} 8 \mathrm{~b}$ & 29.94 & 29.91 & 29.94 & 29.90 \\
\hline H 9a & 29.97 & 30.04 & 29.92 & 29.95 \\
\hline $\mathrm{H} 9 \mathrm{~b}$ & 30.14 & 30.14 & 30.25 & 30.15 \\
\hline H 10 & 29.49 & 29.35 & 29.33 & 29.50 \\
\hline H 11 & 29.73 & 29.66 & 29.67 & 29.76 \\
\hline H $12 a$ & 30.20 & 30.31 & 30.32 & 30.66 \\
\hline $\mathrm{H} 12 \mathrm{~b}$ & 30.59 & 30.31 & 30.28 & 30.24 \\
\hline $\mathrm{H} 13 \mathrm{a}$ & 29.48 & 29.48 & 29.50 & 29.76 \\
\hline $\mathrm{H} 13 \mathrm{~b}$ & 29.72 & 29.52 & 29.49 & 29.49 \\
\hline H 14 & 26.14 & 26.05 & 26.08 & 26.15 \\
\hline H 19a-c & 30.53 & 30.70 & 30.51 & 30.69 \\
\hline H 18a-c & 30.63 & 30.91 & 30.90 & 30.63 \\
\hline H 16a-c & 29.97 & 29.95 & 29.92 & 29.96 \\
\hline H 17a-c & 29.89 & 29.85 & 29.87 & 29.91 \\
\hline H 20a-c & 29.73 & 29.77 & 29.76 & 29.74 \\
\hline
\end{tabular}


Table S8. Boltzmann averaged GIAO isotropic magnetic shielding constants $(\sigma)$ of isomers 1-4 calculated at the PCM/mPW1PW91/6-31+G**//B3LYP/6-31G* level of theory for compound isoelisabethatriene in benzene

\begin{tabular}{|c|c|c|c|c|}
\hline Nuclei & Isomer 1 & Isomer 2 & Isomer 3 & Isomer 4 \\
\hline C 1 & 64.18 & 64.60 & 64.71 & 64.63 \\
\hline$C 2$ & 75.49 & 75.23 & 75.41 & 74.62 \\
\hline C 3 & 62.56 & 63.55 & 62.88 & 63.39 \\
\hline C 4 & 165.05 & 165.09 & 165.01 & 164.99 \\
\hline$C 5$ & 164.14 & 165.92 & 164.23 & 165.29 \\
\hline C 6 & 61.02 & 61.85 & 61.41 & 61.50 \\
\hline C 7 & 159.00 & 158.86 & 159.00 & 158.96 \\
\hline C 8 & 165.15 & 162.33 & 165.27 & 162.94 \\
\hline C 9 & 175.61 & 172.84 & 176.40 & 172.67 \\
\hline C 10 & 149.65 & 152.38 & 152.36 & 149.95 \\
\hline C 11 & 159.62 & 159.45 & 160.19 & 158.23 \\
\hline C 12 & 163.92 & 158.40 & 158.64 & 163.00 \\
\hline C 13 & 165.72 & 166.12 & 166.10 & 165.43 \\
\hline C 14 & 71.39 & 71.43 & 71.20 & 70.98 \\
\hline C 15 & 64.77 & 64.35 & 64.84 & 65.13 \\
\hline C 16 & 177.71 & 177.78 & 177.73 & 177.75 \\
\hline C 17 & 168.90 & 168.87 & 168.78 & 168.83 \\
\hline C 18 & 177.14 & 181.28 & 181.46 & 177.15 \\
\hline C 19 & 174.51 & 176.05 & 174.32 & 176.04 \\
\hline C 20 & 171.10 & 171.49 & 171.24 & 171.66 \\
\hline $\mathrm{H} 2$ & 25.51 & 25.48 & 25.55 & 25.51 \\
\hline $\mathrm{H} 4 \mathrm{a}$ & 29.65 & 29.40 & 29.71 & 29.43 \\
\hline $\mathrm{H} 4 \mathrm{~b}$ & 29.40 & 29.68 & 29.37 & 29.70 \\
\hline H $5 a$ & 29.45 & 29.49 & 29.35 & 29.56 \\
\hline $\mathrm{H} 5 \mathrm{~b}$ & 29.50 & 29.33 & 29.57 & 29.32 \\
\hline H 7 & 29.65 & 29.25 & 29.68 & 29.27 \\
\hline H $8 a$ & 30.18 & 30.41 & 30.15 & 30.43 \\
\hline $\mathrm{H} 8 \mathrm{~b}$ & 29.95 & 29.89 & 29.95 & 29.89 \\
\hline H 9a & 29.97 & 30.02 & 29.91 & 29.94 \\
\hline $\mathrm{H} 9 \mathrm{~b}$ & 30.13 & 30.14 & 30.24 & 30.15 \\
\hline H 10 & 29.47 & 29.33 & 29.31 & 29.48 \\
\hline H 11 & 29.72 & 29.65 & 29.66 & 29.75 \\
\hline H $12 a$ & 30.24 & 30.30 & 30.32 & 30.65 \\
\hline $\mathrm{H} 12 \mathrm{~b}$ & 30.58 & 30.31 & 30.28 & 30.28 \\
\hline H 13a & 29.46 & 29.46 & 29.49 & 29.76 \\
\hline $\mathrm{H} 13 \mathrm{~b}$ & 29.72 & 29.51 & 29.48 & 29.47 \\
\hline H 14 & 26.11 & 26.02 & 26.06 & 26.13 \\
\hline H 16a-c & 29.94 & 29.93 & 29.89 & 29.94 \\
\hline H 17a-c & 29.87 & 29.83 & 29.86 & 29.89 \\
\hline H 18a-c & 30.62 & 30.92 & 30.91 & 30.61 \\
\hline H 19a-c & 30.52 & 30.69 & 30.51 & 30.68 \\
\hline H 20a-c & 29.72 & 29.74 & 29.74 & 29.72 \\
\hline
\end{tabular}


Table S9. Unscaled and scaled chemical shifts and its errors computed for isomers 1-8 at the PCM/mPW1PW91/6-31+G**//B3LYP/6-31G* level of theory for compound 1.

\begin{tabular}{|c|c|c|c|c|c|c|c|c|}
\hline \multicolumn{9}{|c|}{ Unscaled chemical shifts constants } \\
\hline Nuclei & Isomer 1 & Isomer 2 & Isomer 3 & Isomer 4 & Isomer 5 & Isomer 6 & Isomer 7 & Isomer 8 \\
\hline C 1 & 35.28 & 34.06 & 34.02 & 35.31 & 35.49 & 33.66 & 34.35 & 35.60 \\
\hline $\mathrm{C} 2$ & 37.19 & 31.67 & 33.50 & 34.04 & 36.89 & 31.80 & 33.68 & 34.24 \\
\hline C 3 & 26.95 & 23.22 & 24.30 & 29.34 & 26.66 & 22.35 & 24.69 & 28.78 \\
\hline C 4 & 42.66 & 44.54 & 42.96 & 45.66 & 41.88 & 45.22 & 42.84 & 47.01 \\
\hline C 5 & 40.51 & 43.64 & 34.27 & 40.57 & 40.62 & 42.72 & 34.73 & 40.23 \\
\hline C 6 & 146.06 & 146.67 & 146.95 & 146.77 & 145.78 & 147.19 & 146.86 & 147.05 \\
\hline$C 7$ & 120.35 & 120.11 & 120.58 & 120.42 & 120.37 & 120.04 & 120.59 & 120.37 \\
\hline C 8 & 41.79 & 44.45 & 46.77 & 43.97 & 42.40 & 44.56 & 46.29 & 43.79 \\
\hline C 9 & 36.75 & 38.80 & 38.16 & 39.06 & 36.51 & 38.85 & 37.65 & 38.69 \\
\hline C 10 & 39.62 & 36.53 & 38.53 & 38.38 & 39.31 & 37.38 & 37.89 & 38.95 \\
\hline C 11 & 41.12 & 41.09 & 40.69 & 40.57 & 40.90 & 40.49 & 41.13 & 40.86 \\
\hline C 12 & 18.10 & 18.08 & 18.26 & 18.11 & 18.39 & 17.15 & 17.89 & 17.67 \\
\hline C 13 & 36.45 & 35.54 & 36.68 & 36.13 & 36.52 & 36.52 & 37.01 & 36.03 \\
\hline C 14 & 29.08 & 30.05 & 29.30 & 29.75 & 28.81 & 30.23 & 29.17 & 30.13 \\
\hline C 15 & 125.44 & 125.42 & 125.36 & 125.47 & 125.70 & 125.27 & 125.50 & 125.26 \\
\hline C 16 & 132.08 & 132.57 & 132.20 & 132.48 & 131.80 & 132.80 & 131.96 & 132.68 \\
\hline C 17 & 27.49 & 27.73 & 27.61 & 27.53 & 27.65 & 27.54 & 27.57 & 27.51 \\
\hline C 18 & 18.76 & 19.01 & 18.74 & 18.90 & 18.72 & 18.97 & 18.59 & 18.92 \\
\hline C 19 & 21.12 & 19.05 & 19.49 & 20.69 & 20.89 & 19.04 & 19.97 & 20.43 \\
\hline C 20 & 19.26 & 19.09 & 19.16 & 19.20 & 19.20 & 19.01 & 19.33 & 19.06 \\
\hline H 1 & 1.89 & 2.04 & 1.96 & 1.85 & 1.88 & 2.06 & 1.96 & 1.81 \\
\hline $\mathrm{H} 2 \mathrm{a}$ & 1.46 & 1.30 & 1.47 & 1.55 & 1.46 & 1.30 & 1.52 & 1.55 \\
\hline $\mathrm{H} 2 \mathrm{~b}$ & 1.03 & 1.28 & 1.09 & 0.65 & 1.05 & 1.28 & 1.13 & 0.67 \\
\hline H $3 a$ & 1.48 & 1.31 & 1.30 & 1.25 & 1.47 & 1.31 & 1.24 & 1.26 \\
\hline $\mathrm{H} 3 \mathrm{~b}$ & 0.79 & 0.95 & 0.92 & 1.05 & 0.84 & 0.93 & 1.05 & 1.14 \\
\hline H 4 & 1.67 & 1.54 & 1.58 & 1.26 & 1.70 & 1.51 & 1.62 & 1.28 \\
\hline H 5 & 1.40 & 1.35 & 1.57 & 1.36 & 1.47 & 1.33 & 1.63 & 1.31 \\
\hline H 7 & 5.26 & 5.27 & 5.31 & 5.29 & 5.28 & 5.27 & 5.33 & 5.31 \\
\hline H 8a & 2.78 & 2.47 & 2.53 & 2.63 & 2.76 & 2.49 & 2.49 & 2.64 \\
\hline $\mathrm{H} 8 \mathrm{~b}$ & 2.28 & 2.36 & 2.43 & 2.24 & 2.30 & 2.38 & 2.42 & 2.28 \\
\hline H 10 & 0.44 & 0.21 & 0.55 & 0.25 & 0.41 & 0.25 & 0.55 & 0.27 \\
\hline H 11 & 1.49 & 1.53 & 1.47 & 1.53 & 1.51 & 1.52 & 1.54 & 1.49 \\
\hline H $12 a-c$ & 0.92 & 0.91 & 0.93 & 0.90 & 1.01 & 0.91 & 1.01 & 0.94 \\
\hline H 13a & 1.48 & 1.49 & 1.47 & 1.48 & 1.51 & 1.41 & 1.45 & 1.43 \\
\hline $\mathrm{H} 13 \mathrm{~b}$ & 1.37 & 1.21 & 1.39 & 1.16 & 1.21 & 1.32 & 1.29 & 1.27 \\
\hline H 14a & 2.21 & 2.14 & 2.17 & 2.16 & 2.13 & 2.19 & 2.10 & 2.13 \\
\hline H 14b & 2.00 & 2.10 & 2.04 & 2.07 & 2.03 & 2.04 & 2.08 & 2.12 \\
\hline H 15 & 5.46 & 5.47 & 5.51 & 5.45 & 5.47 & 5.48 & 5.51 & 5.47 \\
\hline H 17a-c & 1.72 & 1.73 & 1.71 & 1.73 & 1.71 & 1.73 & 1.71 & 1.71 \\
\hline H 18a-c & 1.70 & 1.68 & 1.69 & 1.67 & 1.69 & 1.68 & 1.67 & 1.69 \\
\hline H 19a-c & 0.99 & 1.02 & 0.80 & 0.86 & 1.00 & 1.03 & 0.81 & 0.84 \\
\hline H 20a-c & 1.84 & 1.83 & 1.83 & 1.83 & 1.85 & 1.79 & 1.81 & 1.80 \\
\hline
\end{tabular}


Scaled chemical shifts constants

\begin{tabular}{|c|c|c|c|c|c|c|c|c|}
\hline Nuclei & Isomer 1 & Isomer 2 & Isomer 3 & Isomer 4 & Isomer 5 & Isomer 6 & Isomer 7 & Isomer 8 \\
\hline C 1 & 32.99 & 32.14 & 32.32 & 32.73 & 33.29 & 31.81 & 32.63 & 33.00 \\
\hline$C 2$ & 34.94 & 29.72 & 31.79 & 31.44 & 34.71 & 29.94 & 31.95 & 31.62 \\
\hline C 3 & 24.51 & 21.18 & 22.50 & 26.66 & 24.28 & 20.41 & 22.87 & 26.07 \\
\hline C 4 & 40.52 & 42.74 & 41.34 & 43.27 & 39.81 & 43.47 & 41.21 & 44.60 \\
\hline$C 5$ & 38.33 & 41.83 & 32.57 & 38.08 & 38.52 & 40.95 & 33.02 & 37.70 \\
\hline C 6 & 145.93 & 146.01 & 146.32 & 146.18 & 145.75 & 146.28 & 146.30 & 146.31 \\
\hline$C 7$ & 119.72 & 119.15 & 119.70 & 119.36 & 119.84 & 118.91 & 119.75 & 119.18 \\
\hline C 8 & 39.63 & 42.64 & 45.19 & 41.55 & 40.33 & 42.80 & 44.70 & 41.33 \\
\hline C 9 & 34.49 & 36.93 & 36.50 & 36.55 & 34.33 & 37.04 & 35.97 & 36.14 \\
\hline C 10 & 37.42 & 34.63 & 36.88 & 35.85 & 37.18 & 35.56 & 36.20 & 36.40 \\
\hline C 11 & 38.95 & 39.25 & 39.05 & 38.09 & 38.80 & 38.70 & 39.48 & 38.35 \\
\hline C 12 & 15.48 & 15.98 & 16.41 & 15.23 & 15.85 & 15.17 & 16.00 & 14.77 \\
\hline C 13 & 34.19 & 33.64 & 35.00 & 33.56 & 34.33 & 34.70 & 35.32 & 33.44 \\
\hline C 14 & 26.67 & 28.08 & 27.55 & 27.08 & 26.47 & 28.35 & 27.39 & 27.44 \\
\hline C 15 & 124.91 & 124.53 & 124.52 & 124.51 & 125.27 & 124.18 & 124.72 & 124.15 \\
\hline C 16 & 131.68 & 131.75 & 131.43 & 131.64 & 131.49 & 131.76 & 131.25 & 131.70 \\
\hline C 17 & 25.05 & 25.74 & 25.85 & 24.82 & 25.29 & 25.64 & 25.78 & 24.78 \\
\hline C 18 & 16.16 & 16.92 & 16.90 & 16.03 & 16.18 & 17.01 & 16.71 & 16.04 \\
\hline C 19 & 18.56 & 16.96 & 17.65 & 17.85 & 18.40 & 17.08 & 18.10 & 17.57 \\
\hline C 20 & 16.66 & 17.00 & 17.32 & 16.33 & 16.68 & 17.04 & 17.45 & 16.18 \\
\hline H 1 & 1.74 & 1.90 & 1.80 & 1.76 & 1.73 & 1.92 & 1.79 & 1.72 \\
\hline H 2a & 1.33 & 1.19 & 1.33 & 1.48 & 1.32 & 1.18 & 1.36 & 1.47 \\
\hline $\mathrm{H} 2 \mathrm{~b}$ & 0.91 & 1.17 & 0.96 & 0.64 & 0.92 & 1.17 & 0.99 & 0.65 \\
\hline H $3 a$ & 1.34 & 1.20 & 1.16 & 1.20 & 1.33 & 1.20 & 1.10 & 1.20 \\
\hline $\mathrm{H} 3 \mathrm{~b}$ & 0.68 & 0.85 & 0.80 & 1.02 & 0.72 & 0.84 & 0.91 & 1.09 \\
\hline H 4 & 1.52 & 1.42 & 1.43 & 1.21 & 1.55 & 1.39 & 1.46 & 1.22 \\
\hline H 5 & 1.27 & 1.23 & 1.43 & 1.30 & 1.33 & 1.22 & 1.47 & 1.25 \\
\hline H 7 & 4.99 & 5.01 & 5.03 & 4.97 & 5.00 & 5.01 & 5.06 & 4.98 \\
\hline H $8 \mathrm{a}$ & 2.59 & 2.32 & 2.35 & 2.48 & 2.57 & 2.33 & 2.31 & 2.49 \\
\hline $\mathrm{H} 8 \mathrm{~b}$ & 2.11 & 2.21 & 2.25 & 2.13 & 2.13 & 2.23 & 2.24 & 2.15 \\
\hline H 10 & 0.33 & 0.14 & 0.45 & 0.27 & 0.31 & 0.18 & 0.43 & 0.28 \\
\hline H 11 & 1.35 & 1.41 & 1.33 & 1.46 & 1.37 & 1.40 & 1.38 & 1.42 \\
\hline H 12a-c & 0.80 & 0.81 & 0.81 & 0.88 & 0.88 & 0.81 & 0.87 & 0.90 \\
\hline H 13a & 1.34 & 1.37 & 1.33 & 1.41 & 1.37 & 1.29 & 1.29 & 1.36 \\
\hline H 13b & 1.24 & 1.10 & 1.25 & 1.12 & 1.08 & 1.20 & 1.15 & 1.21 \\
\hline H 14a & 2.04 & 2.00 & 2.00 & 2.05 & 1.96 & 2.04 & 1.93 & 2.01 \\
\hline $\mathrm{H} 14 \mathrm{~b}$ & 1.84 & 1.96 & 1.88 & 1.96 & 1.87 & 1.90 & 1.91 & 2.00 \\
\hline H 15 & 5.17 & 5.20 & 5.22 & 5.12 & 5.19 & 5.21 & 5.24 & 5.13 \\
\hline H 17a-c & 1.58 & 1.60 & 1.56 & 1.65 & 1.56 & 1.61 & 1.55 & 1.62 \\
\hline H 18a-c & 1.55 & 1.56 & 1.55 & 1.59 & 1.54 & 1.56 & 1.51 & 1.60 \\
\hline H 19a-c & 0.87 & 0.92 & 0.69 & 0.84 & 0.87 & 0.93 & 0.68 & 0.81 \\
\hline H 20a-c & 1.69 & 1.70 & 1.67 & 1.74 & 1.70 & 1.66 & 1.64 & 1.70 \\
\hline
\end{tabular}


Absolute unscaled error

Absolute scaled error

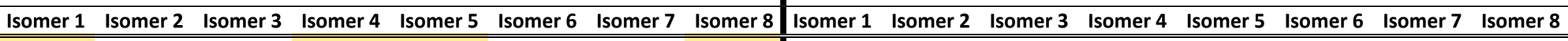

\begin{tabular}{|c|c|c|c|c|c|c|c|c|c|c|c|c|c|c|c|c|}
\hline C 1 & 3.9 & 2.7 & 2.6 & 3.9 & 4.1 & 2.3 & 3.0 & 4.2 & 1.6 & 0.7 & 0.9 & 1.3 & 1.9 & 0.4 & 1.2 & 1.6 \\
\hline C 2 & 5.6 & 0.1 & 1.9 & 2.4 & 5.3 & 0.2 & 2.1 & 2.6 & 3.3 & 1.9 & 0.2 & 0.2 & 3.1 & 1.7 & 0.3 & 0.0 \\
\hline C 3 & 1.5 & 2.3 & 1.2 & 3.8 & 1.2 & 3.2 & 0.8 & 3.3 & 1.0 & 4.3 & 3.0 & 1.2 & 1.2 & 5.1 & 2.6 & 0.6 \\
\hline C 4 & 0.3 & 1.5 & 0.0 & 2.7 & 1.1 & 2.2 & 0.2 & 4.0 & 2.5 & 0.3 & 1.7 & 0.3 & 3.2 & 0.5 & 1.8 & 1.6 \\
\hline C 5 & 4.2 & 7.3 & 2.0 & 4.3 & 4.3 & 6.4 & 1.6 & 3.9 & 2.0 & 5.5 & 3.7 & 1.8 & 2.2 & 4.6 & 3.3 & 1.4 \\
\hline C 6 & 0.2 & 0.8 & 1.0 & 0.9 & 0.1 & 1.3 & 1.0 & 1.2 & 0.0 & 0.1 & 0.4 & 0.3 & 0.1 & 0.4 & 0.4 & 0.4 \\
\hline C 7 & 0.1 & 0.3 & 0.2 & 0.0 & 0.0 & 0.4 & 0.2 & 0.0 & 0.7 & 1.2 & 0.7 & 1.0 & 0.6 & 1.5 & 0.6 & 1.2 \\
\hline C 8 & 1.4 & 4.0 & 6.4 & 3.6 & 2.0 & 4.2 & 5.9 & 3.4 & 0.8 & 2.2 & 4.8 & 1.1 & 0.1 & 2.4 & 4.3 & 0.9 \\
\hline C 9 & 2.0 & 4.1 & 3.5 & 4.4 & 1.8 & 4.1 & 3.0 & 4.0 & 0.2 & 2.2 & 1.8 & 1.8 & 0.4 & 2.3 & 1.3 & 1.4 \\
\hline C 10 & 4.2 & 1.1 & 3.1 & 3.0 & 3.9 & 2.0 & 2.5 & 3.5 & 2.0 & 0.8 & 1.5 & 0.5 & 1.8 & 0.2 & 0.8 & 1.0 \\
\hline C 11 & 2.8 & 2.8 & 2.4 & 2.3 & 2.6 & 2.2 & 2.8 & 2.6 & 0.6 & 0.9 & 0.8 & 0.2 & 0.5 & 0.4 & 1.2 & 0.0 \\
\hline C 12 & 1.7 & 1.7 & 1.9 & 1.7 & 2.0 & 0.8 & 1.5 & 1.3 & 0.9 & 0.4 & 0.0 & 1.2 & 0.6 & 1.2 & 0.4 & 1.6 \\
\hline C 13 & 2.0 & 1.0 & 2.2 & 1.6 & 2.0 & 2.0 & 2.5 & 1.5 & 0.3 & 0.9 & 0.5 & 0.9 & 0.2 & 0.2 & 0.8 & 1.1 \\
\hline C 14 & 2.9 & 3.9 & 3.1 & 3.6 & 2.6 & 4.0 & 3.0 & 3.9 & 0.5 & 1.9 & 1.4 & 0.9 & 0.3 & 2.2 & 1.2 & 1.2 \\
\hline C 15 & 0.1 & 0.1 & 0.1 & 0.2 & 0.4 & 0.0 & 0.2 & 0.0 & 0.4 & 0.8 & 0.8 & 0.8 & 0.0 & 1.1 & 0.6 & 1.1 \\
\hline C 16 & 0.9 & 1.4 & 1.0 & 1.3 & 0.6 & 1.6 & 0.8 & 1.5 & 0.5 & 0.5 & 0.2 & 0.4 & 0.3 & 0.6 & 0.0 & 0.5 \\
\hline C 17 & 1.6 & 1.8 & 1.7 & 1.6 & 1.7 & 1.6 & 1.7 & 1.6 & 0.8 & 0.2 & 0.0 & 1.1 & 0.6 & 0.3 & 0.1 & 1.1 \\
\hline C 18 & 1.0 & 1.2 & 0.9 & 1.1 & 0.9 & 1.2 & 0.8 & 1.1 & 1.6 & 0.9 & 0.9 & 1.8 & 1.6 & 0.8 & 1.1 & 1.8 \\
\hline C 19 & 1.3 & 0.8 & 0.3 & 0.9 & 1.1 & 0.8 & 0.2 & 0.6 & 1.2 & 2.8 & 2.1 & 1.9 & 1.4 & 2.7 & 1.7 & 2.2 \\
\hline C 20 & 2.5 & 2.3 & 2.4 & 2.4 & 2.4 & 2.2 & 2.5 & 2.3 & 0.1 & 0.2 & 0.5 & 0.5 & 0.1 & 0.2 & 0.7 & 0.6 \\
\hline Average & 2.0 & 2.1 & 1.9 & 2.3 & 2.0 & 2.1 & 1.8 & 2.3 & 1.1 & 1.4 & 1.3 & 1.0 & 1.0 & 1.4 & 1.2 & 1.1 \\
\hline H 1 & 0.08 & 0.23 & 0.15 & 0.04 & 0.07 & 0.25 & 0.15 & 0.00 & 0.07 & 0.09 & 0.01 & 0.05 & 0.08 & 0.11 & 0.02 & 0.09 \\
\hline $\mathrm{H} 2 \mathrm{a}$ & 0.14 & 0.30 & 0.13 & 0.05 & 0.14 & 0.30 & 0.08 & 0.05 & 0.27 & 0.41 & 0.27 & 0.12 & 0.28 & 0.42 & 0.24 & 0.13 \\
\hline $\mathrm{H} 2 \mathrm{~b}$ & 0.52 & 0.77 & 0.58 & 0.14 & 0.54 & 0.77 & 0.62 & 0.16 & 0.40 & 0.66 & 0.45 & 0.13 & 0.41 & 0.66 & 0.48 & 0.14 \\
\hline H 3a & 0.15 & 0.02 & 0.03 & 0.08 & 0.14 & 0.02 & 0.09 & 0.07 & 0.01 & 0.13 & 0.17 & 0.13 & 0.00 & 0.13 & 0.23 & 0.13 \\
\hline$H 3 b$ & 0.09 & 0.07 & 0.04 & 0.17 & 0.04 & 0.05 & 0.17 & 0.26 & 0.20 & 0.03 & 0.08 & 0.14 & 0.16 & 0.04 & 0.03 & 0.21 \\
\hline $\mathrm{H} 4$ & 0.45 & 0.32 & 0.36 & 0.04 & 0.48 & 0.29 & 0.40 & 0.06 & 0.30 & 0.20 & 0.21 & 0.01 & 0.33 & 0.17 & 0.24 & 0.00 \\
\hline H 5 & 0.25 & 0.20 & 0.42 & 0.21 & 0.32 & 0.18 & 0.48 & 0.16 & 0.12 & 0.08 & 0.28 & 0.15 & 0.18 & 0.07 & 0.32 & 0.10 \\
\hline H 7 & 0.37 & 0.38 & 0.42 & 0.40 & 0.39 & 0.38 & 0.44 & 0.42 & 0.10 & 0.12 & 0.14 & 0.08 & 0.11 & 0.12 & 0.17 & 0.09 \\
\hline H 8a & 0.27 & 0.04 & 0.02 & 0.12 & 0.25 & 0.02 & 0.02 & 0.13 & 0.08 & 0.19 & 0.16 & 0.03 & 0.06 & 0.18 & 0.20 & 0.02 \\
\hline $\mathrm{H} 8 \mathrm{~b}$ & 0.13 & 0.21 & 0.28 & 0.09 & 0.15 & 0.23 & 0.27 & 0.13 & 0.04 & 0.06 & 0.10 & 0.02 & 0.02 & 0.08 & 0.09 & 0.00 \\
\hline H 10 & 0.23 & 0.00 & 0.34 & 0.04 & 0.20 & 0.04 & 0.34 & 0.06 & 0.12 & 0.07 & 0.24 & 0.06 & 0.10 & 0.03 & 0.22 & 0.07 \\
\hline H 11 & 0.03 & 0.07 & 0.01 & 0.07 & 0.05 & 0.06 & 0.08 & 0.03 & 0.11 & 0.05 & 0.13 & 0.00 & 0.09 & 0.06 & 0.08 & 0.04 \\
\hline H 12a-c & 0.04 & 0.03 & 0.05 & 0.02 & 0.13 & 0.03 & 0.13 & 0.06 & 0.08 & 0.07 & 0.07 & 0.00 & 0.00 & 0.07 & 0.01 & 0.02 \\
\hline H 13a & 0.03 & 0.04 & 0.02 & 0.03 & 0.06 & 0.04 & 0.00 & 0.02 & 0.11 & 0.08 & 0.12 & 0.04 & 0.08 & 0.16 & 0.16 & 0.09 \\
\hline H 13b & 0.19 & 0.03 & 0.21 & 0.02 & 0.03 & 0.14 & 0.11 & 0.09 & 0.06 & 0.08 & 0.07 & 0.06 & 0.10 & 0.02 & 0.03 & 0.03 \\
\hline H 14a & 0.18 & 0.11 & 0.14 & 0.13 & 0.10 & 0.16 & 0.07 & 0.10 & 0.01 & 0.03 & 0.03 & 0.02 & 0.07 & 0.01 & 0.10 & 0.02 \\
\hline H 14b & 0.08 & 0.18 & 0.12 & 0.15 & 0.11 & 0.12 & 0.16 & 0.20 & 0.08 & 0.04 & 0.04 & 0.04 & 0.05 & 0.02 & 0.01 & 0.08 \\
\hline H 15 & 0.35 & 0.36 & 0.40 & 0.34 & 0.36 & 0.37 & 0.40 & 0.36 & 0.06 & 0.09 & 0.11 & 0.01 & 0.08 & 0.10 & 0.13 & 0.02 \\
\hline H 17a-c & 0.03 & 0.04 & 0.02 & 0.04 & 0.02 & 0.04 & 0.02 & 0.02 & 0.11 & 0.09 & 0.13 & 0.04 & 0.13 & 0.08 & 0.14 & 0.07 \\
\hline H 18a-c & 0.09 & 0.07 & 0.08 & 0.06 & 0.08 & 0.07 & 0.06 & 0.08 & 0.06 & 0.05 & 0.06 & 0.02 & 0.07 & 0.05 & 0.10 & 0.01 \\
\hline$-19 a-c$ & 0.07 & 0.10 & 0.12 & 0.06 & 0.08 & 0.11 & 0.11 & 0.08 & 0.05 & 0.00 & 0.23 & 0.08 & 0.05 & 0.01 & 0.24 & 0.11 \\
\hline H 2Oa-c & 0.08 & 0.07 & 0.07 & 0.07 & 0.09 & 0.03 & 0.05 & 0.04 & 0.07 & 0.06 & 0.09 & 0.02 & 0.06 & 0.10 & 0.12 & 0.06 \\
\hline Average & 0.18 & 0.17 & 0.18 & 0.11 & 0.17 & 0.17 & 0.19 & 0.12 & 0.11 & 0.12 & 0.14 & 0.06 & 0.11 & 0.12 & 0.15 & 0.07 \\
\hline
\end{tabular}


Table S10. Unscaled and scaled chemical shifts and its errors computed for isomers $1-8$ at the PCM/mPW1PW91/6-31+G**//B3LYP/6-31G* level of theory for compound 2

\begin{tabular}{|c|c|c|c|c|c|c|c|c|}
\hline \multicolumn{9}{|c|}{ Unscaled chemical shifts constants } \\
\hline Nuclei & Isomer 1 & Isomer 2 & Isomer 3 & Isomer 4 & Isomer 5 & Isomer 6 & Isomer 7 & Isomer 8 \\
\hline C 1 & 29.89 & 32.13 & 30.13 & 31.96 & 30.12 & 31.90 & 30.48 & 31.72 \\
\hline $\mathrm{C} 2$ & 36.20 & 34.62 & 36.25 & 34.35 & 35.77 & 34.34 & 35.53 & 34.75 \\
\hline C 3 & 116.64 & 114.15 & 117.24 & 114.81 & 117.04 & 114.94 & 117.50 & 113.71 \\
\hline $\mathrm{C} 4$ & 144.51 & 145.16 & 144.64 & 143.49 & 144.05 & 144.08 & 143.53 & 144.62 \\
\hline $\mathrm{C} 5$ & 42.47 & 44.26 & 42.28 & 44.97 & 42.49 & 44.52 & 42.75 & 44.96 \\
\hline C 6 & 154.70 & 154.31 & 154.82 & 154.56 & 155.01 & 154.69 & 154.26 & 154.54 \\
\hline C 7 & 31.92 & 32.24 & 31.95 & 31.87 & 31.53 & 32.09 & 31.62 & 31.84 \\
\hline C 8 & 30.60 & 33.91 & 31.84 & 34.29 & 31.21 & 34.45 & 31.70 & 34.53 \\
\hline C 9 & 45.14 & 44.82 & 45.29 & 44.93 & 45.13 & 44.76 & 45.28 & 45.19 \\
\hline C 10 & 27.82 & 26.94 & 29.47 & 28.30 & 28.85 & 29.21 & 29.47 & 28.01 \\
\hline C 11 & 45.51 & 45.78 & 45.75 & 45.87 & 45.52 & 45.55 & 45.92 & 44.96 \\
\hline C 12 & 22.24 & 22.01 & 21.80 & 21.77 & 21.65 & 22.19 & 21.07 & 20.87 \\
\hline C 13 & 34.61 & 33.90 & 33.54 & 33.42 & 33.64 & 33.27 & 32.46 & 32.67 \\
\hline C 14 & 31.16 & 31.11 & 31.10 & 31.36 & 31.05 & 30.96 & 31.06 & 30.64 \\
\hline C 15 & 65.77 & 65.79 & 65.59 & 65.98 & 65.55 & 65.67 & 65.21 & 65.48 \\
\hline C 16 & 59.96 & 60.14 & 60.22 & 60.30 & 60.20 & 60.18 & 60.14 & 60.17 \\
\hline C 17 & 20.09 & 20.41 & 19.55 & 19.78 & 25.67 & 25.74 & 25.85 & 25.93 \\
\hline C 18 & 25.75 & 25.67 & 25.89 & 25.76 & 19.57 & 19.73 & 19.55 & 19.65 \\
\hline C 19 & 19.91 & 19.24 & 19.93 & 20.07 & 19.99 & 19.62 & 20.17 & 20.01 \\
\hline C 20 & 99.80 & 100.19 & 99.67 & 99.88 & 99.73 & 100.13 & 99.37 & 99.61 \\
\hline H 1 & 1.88 & 2.06 & 1.87 & 2.07 & 1.87 & 2.04 & 1.86 & 2.13 \\
\hline $\mathrm{H} 2 \mathrm{a}$ & 1.84 & 2.35 & 1.98 & 2.27 & 1.91 & 2.32 & 1.99 & 2.36 \\
\hline $\mathrm{H} 2 \mathrm{~b}$ & 1.76 & 1.71 & 1.75 & 1.69 & 1.77 & 1.75 & 1.73 & 1.73 \\
\hline H 3 & 5.78 & 5.57 & 5.59 & 5.55 & 5.69 & 5.51 & 5.58 & 5.58 \\
\hline H 5 & 2.04 & 2.04 & 2.00 & 1.92 & 2.08 & 2.04 & 2.03 & 2.01 \\
\hline H 7a & 2.27 & 2.17 & 2.29 & 2.25 & 2.27 & 2.22 & 2.25 & 2.28 \\
\hline $\mathrm{H} 7 \mathrm{~b}$ & 2.15 & 2.15 & 2.23 & 2.21 & 2.18 & 2.19 & 2.21 & 2.23 \\
\hline $\mathrm{H} 8 \mathrm{a}$ & 2.17 & 1.95 & 2.24 & 1.94 & 2.22 & 1.95 & 2.26 & 1.94 \\
\hline $\mathrm{H} 8 \mathrm{~b}$ & 1.66 & 1.81 & 1.57 & 1.91 & 1.67 & 1.84 & 1.52 & 1.89 \\
\hline H 10 & 1.57 & 1.54 & 1.53 & 1.61 & 1.53 & 1.51 & 1.56 & 1.62 \\
\hline H 11 & 2.15 & 2.16 & 2.15 & 2.11 & 2.07 & 2.13 & 2.11 & 2.15 \\
\hline H 12a-c & 1.09 & 1.08 & 1.05 & 1.05 & 1.10 & 1.09 & 1.06 & 1.04 \\
\hline H 13a & 1.57 & 1.57 & 1.57 & 1.67 & 1.56 & 1.59 & 1.50 & 1.50 \\
\hline $\mathrm{H} 13 \mathrm{~b}$ & 1.48 & 1.46 & 1.25 & 1.27 & 1.25 & 1.29 & 1.37 & 1.39 \\
\hline H 14a & 1.54 & 1.43 & 1.69 & 1.61 & 1.74 & 1.66 & 1.74 & 1.75 \\
\hline $\mathrm{H} 14 \mathrm{~b}$ & 1.14 & 1.27 & 1.11 & 1.27 & 1.14 & 1.22 & 1.07 & 1.11 \\
\hline H 15 & 2.64 & 2.62 & 2.59 & 2.64 & 2.60 & 2.60 & 2.65 & 2.63 \\
\hline H 17a-c & 1.17 & 1.19 & 1.15 & 1.16 & 1.17 & 1.18 & 1.16 & 1.16 \\
\hline H 18a-c & 1.15 & 1.16 & 1.17 & 1.18 & 1.16 & 1.16 & 1.15 & 1.16 \\
\hline H 19a-c & 0.97 & 1.00 & 1.00 & 0.99 & 0.98 & 0.95 & 1.01 & 1.00 \\
\hline H 20a & 5.13 & 5.14 & 5.17 & 5.18 & 5.20 & 5.18 & 5.16 & 5.15 \\
\hline $\mathrm{H} 20 \mathrm{~b}$ & 4.98 & 5.00 & 4.96 & 4.95 & 4.92 & 4.97 & 4.95 & 4.94 \\
\hline
\end{tabular}


Scaled chemical shifts constants

\begin{tabular}{|c|c|c|c|c|c|c|c|c|}
\hline Nuclei & Isomer 1 & Isomer 2 & Isomer 3 & Isomer 4 & Isomer 5 & Isomer 6 & Isomer 7 & Isomer 8 \\
\hline C 1 & 27.19 & 29.22 & 27.34 & 28.91 & 27.47 & 28.86 & 27.79 & 28.89 \\
\hline$C 2$ & 33.59 & 31.76 & 33.54 & 31.36 & 33.20 & 31.36 & 32.92 & 31.98 \\
\hline C 3 & 115.11 & 112.85 & 115.56 & 113.72 & 115.56 & 113.76 & 116.32 & 112.72 \\
\hline C 4 & 143.36 & 144.47 & 143.31 & 143.08 & 142.94 & 143.55 & 142.81 & 144.33 \\
\hline C 5 & 39.94 & 41.59 & 39.64 & 42.23 & 40.01 & 41.76 & 40.27 & 42.42 \\
\hline C 6 & 153.69 & 153.80 & 153.62 & 154.41 & 154.04 & 154.40 & 153.73 & 154.47 \\
\hline$C 7$ & 29.25 & 29.33 & 29.18 & 28.82 & 28.90 & 29.05 & 28.95 & 29.01 \\
\hline C 8 & 27.91 & 31.03 & 29.07 & 31.29 & 28.58 & 31.47 & 29.03 & 31.76 \\
\hline$C 9$ & 42.65 & 42.16 & 42.69 & 42.19 & 42.68 & 42.01 & 42.84 & 42.66 \\
\hline C 10 & 25.09 & 23.93 & 26.67 & 25.16 & 26.19 & 26.11 & 26.76 & 25.09 \\
\hline C 11 & 43.02 & 43.14 & 43.16 & 43.15 & 43.08 & 42.82 & 43.50 & 42.42 \\
\hline C 12 & 19.44 & 18.90 & 18.90 & 18.48 & 18.89 & 18.93 & 18.21 & 17.79 \\
\hline C 13 & 31.98 & 31.02 & 30.79 & 30.40 & 31.04 & 30.26 & 29.80 & 29.86 \\
\hline C 14 & 28.48 & 28.18 & 28.32 & 28.29 & 28.41 & 27.90 & 28.38 & 27.78 \\
\hline C 15 & 63.56 & 63.54 & 63.25 & 63.73 & 63.38 & 63.39 & 63.12 & 63.41 \\
\hline C 16 & 57.67 & 57.78 & 57.81 & 57.92 & 57.96 & 57.77 & 57.96 & 57.98 \\
\hline C 17 & 17.26 & 17.27 & 16.62 & 16.44 & 22.96 & 22.56 & 23.08 & 22.97 \\
\hline C 18 & 23.00 & 22.63 & 23.04 & 22.56 & 16.78 & 16.42 & 16.67 & 16.55 \\
\hline C 19 & 17.08 & 16.08 & 17.01 & 16.74 & 17.21 & 16.31 & 17.30 & 16.91 \\
\hline C 20 & 98.05 & 98.62 & 97.77 & 98.43 & 98.02 & 98.62 & 97.88 & 98.30 \\
\hline H 1 & 1.82 & 1.97 & 1.82 & 1.98 & 1.82 & 1.95 & 1.81 & 2.02 \\
\hline $\mathrm{H} 2 \mathrm{a}$ & 1.79 & 2.24 & 1.92 & 2.16 & 1.85 & 2.21 & 1.93 & 2.24 \\
\hline $\mathrm{H} 2 \mathrm{~b}$ & 1.72 & 1.65 & 1.71 & 1.63 & 1.72 & 1.69 & 1.69 & 1.65 \\
\hline H 3 & 5.35 & 5.21 & 5.23 & 5.20 & 5.29 & 5.17 & 5.23 & 5.22 \\
\hline H 5 & 1.97 & 1.95 & 1.94 & 1.84 & 2.01 & 1.95 & 1.97 & 1.91 \\
\hline $\mathrm{H} 7 \mathrm{a}$ & 2.18 & 2.07 & 2.21 & 2.14 & 2.18 & 2.12 & 2.17 & 2.16 \\
\hline $\mathrm{H} 7 \mathrm{~b}$ & 2.07 & 2.06 & 2.15 & 2.11 & 2.10 & 2.09 & 2.13 & 2.12 \\
\hline H 8a & 2.09 & 1.87 & 2.16 & 1.86 & 2.13 & 1.87 & 2.18 & 1.85 \\
\hline $\mathrm{H} 8 \mathrm{~b}$ & 1.63 & 1.74 & 1.55 & 1.83 & 1.63 & 1.77 & 1.50 & 1.80 \\
\hline H 10 & 1.54 & 1.49 & 1.51 & 1.55 & 1.51 & 1.46 & 1.53 & 1.55 \\
\hline H 11 & 2.07 & 2.06 & 2.08 & 2.02 & 2.00 & 2.04 & 2.04 & 2.04 \\
\hline H $12 a-c$ & 1.11 & 1.07 & 1.07 & 1.03 & 1.11 & 1.08 & 1.07 & 1.01 \\
\hline H 13a & 1.54 & 1.52 & 1.55 & 1.61 & 1.53 & 1.54 & 1.48 & 1.44 \\
\hline $\mathrm{H} 13 \mathrm{~b}$ & 1.46 & 1.42 & 1.25 & 1.24 & 1.25 & 1.26 & 1.36 & 1.34 \\
\hline H 14a & 1.52 & 1.39 & 1.66 & 1.55 & 1.70 & 1.60 & 1.70 & 1.67 \\
\hline $\mathrm{H} 14 \mathrm{~b}$ & 1.16 & 1.24 & 1.12 & 1.24 & 1.15 & 1.20 & 1.08 & 1.08 \\
\hline H 15 & 2.51 & 2.49 & 2.48 & 2.51 & 2.48 & 2.47 & 2.54 & 2.49 \\
\hline H 17a-c & 1.18 & 1.17 & 1.16 & 1.14 & 1.18 & 1.16 & 1.17 & 1.12 \\
\hline H 18a-c & 1.16 & 1.14 & 1.18 & 1.15 & 1.17 & 1.14 & 1.16 & 1.12 \\
\hline H 19a-c & 1.00 & 0.99 & 1.02 & 0.98 & 1.00 & 0.95 & 1.03 & 0.98 \\
\hline H 20a & 4.76 & 4.81 & 4.84 & 4.86 & 4.85 & 4.86 & 4.84 & 4.82 \\
\hline $\mathrm{H} 20 \mathrm{~b}$ & 4.63 & 4.69 & 4.65 & 4.64 & 4.59 & 4.67 & 4.65 & 4.63 \\
\hline
\end{tabular}


Absolute unscaled error

Absolute scaled error

\begin{tabular}{|c|c|c|c|c|c|c|c|c|c|c|c|c|c|c|c|c|}
\hline & & & & & & & & & \\
\hline & Isomer 1 & Isomer 2 & Isomer 3 & Isomer 4 & Isomer 5 & Isomer 6 & Isomer 7 & Isomer 8 & Isomer 1 & Isomer 2 & Isomer 3 & Isomer 4 & Isomer 5 & Isomer 6 & Isomer 7 & Isomer 8 \\
\hline C 1 & 3.6 & 5.8 & 3.8 & 5.7 & 3.8 & 5.6 & 4.2 & 5.4 & 0.9 & 2.9 & 1.0 & 2.6 & 1.2 & 2.6 & 1.5 & 2.6 \\
\hline C 2 & 4.1 & 2.5 & 4.1 & 2.2 & 3.7 & 2.2 & 3.4 & 2.6 & 1.5 & 0.3 & 1.4 & 0.7 & 1.1 & 0.7 & 0.8 & 0.1 \\
\hline C 3 & 0.1 & 2.4 & 0.7 & 1.7 & 0.5 & 1.6 & 1.0 & 2.8 & 1.4 & 3.6 & 0.9 & 2.8 & 0.9 & 2.7 & 0.2 & 3.8 \\
\hline C 4 & 4.5 & 5.2 & 4.6 & 3.5 & 4.0 & 4.1 & 3.5 & 4.6 & 3.4 & 4.5 & 3.3 & 3.1 & 2.9 & 3.6 & 2.8 & 4.3 \\
\hline C 5 & 5.4 & 7.2 & 5.2 & 7.9 & 5.4 & 7.4 & 5.6 & 7.9 & 2.8 & 4.5 & 2.5 & 5.1 & 2.9 & 4.7 & 3.2 & 5.3 \\
\hline C 6 & 1.0 & 0.6 & 1.1 & 0.9 & 1.3 & 1.0 & 0.6 & 0.8 & 0.0 & 0.1 & 0.1 & 0.7 & 0.3 & 0.7 & 0.0 & 0.8 \\
\hline C 7 & 3.0 & 3.3 & 3.0 & 3.0 & 2.6 & 3.2 & 2.7 & 2.9 & 0.3 & 0.4 & 0.3 & 0.1 & 0.0 & 0.2 & 0.0 & 0.1 \\
\hline C 8 & 1.5 & 4.8 & 2.7 & 5.2 & 2.1 & 5.3 & 2.6 & 5.4 & 1.2 & 1.9 & 0.0 & 2.2 & 0.5 & 2.4 & 0.1 & 2.7 \\
\hline C9 & 5.1 & 4.8 & 5.3 & 4.9 & 5.1 & 4.8 & 5.3 & 5.2 & 2.6 & 2.2 & 2.7 & 2.2 & 2.7 & 2.0 & 2.8 & 2.7 \\
\hline C 10 & 2.1 & 1.2 & 3.8 & 2.6 & 3.1 & 3.5 & 3.8 & 2.3 & 0.6 & 1.8 & 1.0 & 0.5 & 0.5 & 0.4 & 1.1 & 0.6 \\
\hline C 11 & 4.5 & 4.8 & 4.7 & 4.9 & 4.5 & 4.5 & 4.9 & 4.0 & 2.0 & 2.1 & 2.2 & 2.1 & 2.1 & 1.8 & 2.5 & 1.4 \\
\hline C 12 & 2.6 & 2.4 & 2.2 & 2.2 & 2.0 & 2.6 & 1.5 & 1.3 & 0.2 & 0.7 & 0.7 & 1.1 & 0.7 & 0.7 & 1.4 & 1.8 \\
\hline C 13 & 2.8 & 2.1 & 1.7 & 1.6 & 1.8 & 1.5 & 0.7 & 0.9 & 0.2 & 0.8 & 1.0 & 1.4 & 0.8 & 1.5 & 2.0 & 1.9 \\
\hline C 14 & 4.1 & 4.0 & 4.0 & 4.3 & 3.9 & 3.9 & 4.0 & 3.5 & 1.4 & 1.1 & 1.2 & 1.2 & 1.3 & 0.8 & 1.3 & 0.7 \\
\hline C 15 & 1.1 & 1.1 & 0.9 & 1.3 & 0.8 & 1.0 & 0.5 & 0.8 & 1.1 & 1.2 & 1.4 & 1.0 & 1.3 & 1.3 & 1.6 & 1.3 \\
\hline C 16 & 1.6 & 1.7 & 1.8 & 1.9 & 1.8 & 1.8 & 1.7 & 1.8 & 0.7 & 0.6 & 0.6 & 0.5 & 0.4 & 0.6 & 0.4 & 0.4 \\
\hline C 17 & 1.4 & 1.7 & 0.8 & 1.1 & 7.0 & 7.0 & 7.1 & 7.2 & 1.4 & 1.4 & 2.1 & 2.3 & 4.3 & 3.9 & 4.4 & 4.3 \\
\hline C 18 & 0.6 & 0.6 & 0.8 & 0.7 & 5.5 & 5.4 & 5.6 & 5.5 & 2.1 & 2.5 & 2.1 & 2.5 & 8.3 & 8.7 & 8.4 & 8.6 \\
\hline C 19 & 0.7 & 0.0 & 0.7 & 0.9 & 0.8 & 0.4 & 1.0 & 0.8 & 2.1 & 3.1 & 2.2 & 2.5 & 2.0 & 2.9 & 1.9 & 2.3 \\
\hline C 20 & 2.5 & 2.1 & 2.6 & 2.4 & 2.6 & 2.2 & 2.9 & 2.7 & 4.3 & 3.7 & 4.5 & 3.9 & 4.3 & 3.7 & 4.4 & 4.0 \\
\hline Average & 2.6 & 2.9 & 2.7 & 2.9 & 3.1 & 3.4 & 3.1 & 3.4 & 1.5 & 2.0 & 1.6 & 1.9 & 1.9 & 2.3 & 2.0 & 2.5 \\
\hline H 1 & 0.06 & 0.24 & 0.05 & 0.25 & 0.05 & 0.22 & 0.04 & 0.31 & 0.00 & 0.15 & 0.00 & 0.16 & 0.00 & 0.13 & 0.01 & 0.20 \\
\hline $\mathrm{H} 2 \mathrm{a}$ & 0.20 & 0.31 & 0.06 & 0.23 & 0.13 & 0.28 & 0.05 & 0.32 & 0.25 & 0.20 & 0.12 & 0.12 & 0.19 & 0.17 & 0.11 & 0.20 \\
\hline $\mathrm{H} 2 \mathrm{~b}$ & 0.28 & 0.23 & 0.27 & 0.21 & 0.29 & 0.27 & 0.25 & 0.25 & 0.24 & 0.17 & 0.23 & 0.15 & 0.24 & 0.21 & 0.21 & 0.17 \\
\hline $\mathrm{H}_{3}$ & 0.64 & 0.43 & 0.45 & 0.41 & 0.55 & 0.37 & 0.44 & 0.44 & 0.21 & 0.07 & 0.09 & 0.06 & 0.15 & 0.03 & 0.09 & 0.08 \\
\hline $\mathrm{H} 5$ & 0.23 & 0.23 & 0.19 & 0.11 & 0.27 & 0.23 & 0.22 & 0.20 & 0.16 & 0.14 & 0.13 & 0.03 & 0.20 & 0.14 & 0.16 & 0.10 \\
\hline $\mathrm{H} 7 \mathrm{a}$ & 0.16 & 0.06 & 0.18 & 0.14 & 0.16 & 0.11 & 0.14 & 0.17 & 0.07 & 0.04 & 0.10 & 0.03 & 0.07 & 0.01 & 0.06 & 0.05 \\
\hline $\mathrm{H} 7 \mathrm{~b}$ & 0.54 & 0.54 & 0.62 & 0.60 & 0.57 & 0.58 & 0.60 & 0.62 & 0.46 & 0.45 & 0.54 & 0.50 & 0.49 & 0.48 & 0.52 & 0.51 \\
\hline H 8 a & 0.01 & 0.23 & 0.06 & 0.24 & 0.04 & 0.23 & 0.08 & 0.24 & 0.09 & 0.31 & 0.02 & 0.32 & 0.05 & 0.31 & 0.00 & 0.33 \\
\hline H $8 b$ & 0.46 & 0.31 & 0.55 & 0.21 & 0.45 & 0.28 & 0.60 & 0.23 & 0.49 & 0.38 & 0.57 & 0.29 & 0.49 & 0.35 & 0.62 & 0.32 \\
\hline H 10 & 0.32 & 0.29 & 0.28 & 0.36 & 0.28 & 0.26 & 0.31 & 0.37 & 0.29 & 0.24 & 0.26 & 0.30 & 0.26 & 0.21 & 0.28 & 0.30 \\
\hline H 11 & 0.03 & 0.02 & 0.03 & 0.07 & 0.11 & 0.05 & 0.07 & 0.03 & 0.11 & 0.12 & 0.10 & 0.16 & 0.18 & 0.14 & 0.14 & 0.14 \\
\hline H $12 a-c$ & 0.06 & 0.05 & 0.02 & 0.02 & 0.07 & 0.06 & 0.03 & 0.01 & 0.08 & 0.04 & 0.04 & 0.00 & 0.08 & 0.05 & 0.04 & 0.02 \\
\hline H 13a & 0.07 & 0.07 & 0.07 & 0.17 & 0.06 & 0.09 & 0.00 & 0.00 & 0.04 & 0.02 & 0.05 & 0.11 & 0.03 & 0.04 & 0.02 & 0.06 \\
\hline H 13b & 0.08 & 0.06 & 0.15 & 0.13 & 0.15 & 0.11 & 0.03 & 0.01 & 0.06 & 0.02 & 0.15 & 0.16 & 0.15 & 0.14 & 0.04 & 0.06 \\
\hline H 14a & 0.04 & 0.07 & 0.19 & 0.11 & 0.24 & 0.16 & 0.24 & 0.25 & 0.02 & 0.11 & 0.16 & 0.05 & 0.20 & 0.10 & 0.20 & 0.17 \\
\hline H 14b & 0.25 & 0.12 & 0.28 & 0.12 & 0.25 & 0.17 & 0.32 & 0.28 & 0.23 & 0.15 & 0.27 & 0.15 & 0.24 & 0.19 & 0.31 & 0.31 \\
\hline H 15 & 0.08 & 0.10 & 0.13 & 0.08 & 0.12 & 0.12 & 0.07 & 0.09 & 0.21 & 0.23 & 0.24 & 0.21 & 0.24 & 0.25 & 0.18 & 0.23 \\
\hline H 17a-c & 0.07 & 0.05 & 0.09 & 0.08 & 0.07 & 0.06 & 0.08 & 0.08 & 0.06 & 0.07 & 0.08 & 0.10 & 0.06 & 0.08 & 0.07 & 0.12 \\
\hline H 18a-c & 0.15 & 0.14 & 0.13 & 0.12 & 0.14 & 0.14 & 0.15 & 0.14 & 0.14 & 0.16 & 0.12 & 0.15 & 0.13 & 0.16 & 0.14 & 0.18 \\
\hline H 19a-c & 0.07 & 0.04 & 0.04 & 0.05 & 0.06 & 0.09 & 0.03 & 0.04 & 0.04 & 0.05 & 0.02 & 0.06 & 0.04 & 0.09 & 0.01 & 0.06 \\
\hline H 20a & 0.35 & 0.36 & 0.39 & 0.40 & 0.42 & 0.40 & 0.38 & 0.37 & 0.02 & 0.03 & 0.06 & 0.08 & 0.07 & 0.08 & 0.06 & 0.04 \\
\hline H 20b & 0.36 & 0.38 & 0.34 & 0.33 & 0.30 & 0.35 & 0.33 & 0.32 & 0.01 & 0.07 & 0.03 & 0.02 & 0.03 & 0.05 & 0.03 & 0.01 \\
\hline Average & 0.21 & 0.20 & 0.21 & 0.20 & 0.22 & 0.21 & 0.20 & 0.22 & 0.15 & 0.15 & 0.15 & 0.15 & 0.16 & 0.16 & 0.15 & 0.17 \\
\hline
\end{tabular}


Table S11. Unscaled and scaled chemical shifts and its errors computed for isomers 1-16 at the $\mathrm{PCM} / \mathrm{mPW} 1 \mathrm{PW} 91 / 6-31+\mathrm{G}^{* *} / / \mathrm{B} 3 \mathrm{LYP} / 6-31 \mathrm{G}^{*}$ level of theory for compound 3

\begin{tabular}{|c|c|c|c|c|c|c|c|c|}
\hline \multicolumn{9}{|c|}{ Unscaled chemical shifts constants } \\
\hline Nuclei & Isomer 1 & Isomer 2 & Isomer 3 & Isomer 4 & Isomer 5 & Isomer 6 & Isomer 7 & Isomer 8 \\
\hline C 1 & 35.40 & 35.20 & 34.57 & 34.28 & 34.97 & 31.90 & 35.71 & 33.60 \\
\hline C 2 & 28.95 & 29.71 & 32.59 & 30.08 & 30.10 & 27.22 & 36.00 & 29.45 \\
\hline C 3 & 29.05 & 35.19 & 31.73 & 28.23 & 37.29 & 18.74 & 40.46 & 20.83 \\
\hline C 4 & 77.86 & 77.58 & 78.65 & 78.82 & 78.26 & 80.07 & 79.57 & 80.07 \\
\hline C 5 & 35.54 & 35.73 & 39.65 & 39.22 & 36.15 & 36.36 & 42.64 & 40.83 \\
\hline C 6 & 155.61 & 156.39 & 154.52 & 154.23 & 155.32 & 155.30 & 155.43 & 154.91 \\
\hline C 7 & 31.14 & 31.02 & 30.50 & 29.98 & 32.02 & 32.37 & 31.50 & 31.84 \\
\hline C 8 & 33.80 & 34.47 & 33.96 & 33.66 & 32.66 & 36.98 & 30.94 & 34.46 \\
\hline$C 9$ & 43.00 & 44.28 & 42.79 & 42.16 & 43.62 & 40.48 & 45.28 & 38.48 \\
\hline C 10 & 37.24 & 34.98 & 33.68 & 37.61 & 29.92 & 38.98 & 29.86 & 39.05 \\
\hline C 11 & 43.13 & 39.85 & 36.66 & 37.88 & 42.44 & 43.18 & 45.84 & 43.77 \\
\hline C 12 & 17.90 & 16.00 & 16.62 & 15.96 & 18.89 & 18.56 & 19.35 & 18.59 \\
\hline C 13 & 30.43 & 29.85 & 29.98 & 29.30 & 30.07 & 31.40 & 31.83 & 31.05 \\
\hline C 14 & 26.59 & 22.51 & 23.17 & 22.94 & 29.04 & 28.98 & 27.74 & 29.04 \\
\hline C 15 & 77.17 & 77.94 & 78.34 & 77.20 & 77.07 & 76.88 & 77.49 & 76.86 \\
\hline C 16 & 73.92 & 74.12 & 73.80 & 73.67 & 74.73 & 73.53 & 73.31 & 73.48 \\
\hline C 17 & 26.80 & 24.83 & 27.25 & 26.99 & 27.37 & 27.30 & 27.68 & 27.29 \\
\hline C 18 & 25.67 & 27.03 & 23.99 & 24.08 & 24.18 & 24.09 & 24.41 & 23.94 \\
\hline C 19 & 19.78 & 19.57 & 20.82 & 20.48 & 19.66 & 19.45 & 20.20 & 21.64 \\
\hline C 20 & 99.52 & 99.81 & 99.83 & 99.62 & 100.17 & 99.47 & 99.87 & 99.01 \\
\hline H 1 & 1.91 & 1.92 & 2.01 & 1.92 & 1.88 & 2.12 & 1.64 & 2.00 \\
\hline $\mathrm{H} 2 \mathrm{a}$ & 1.27 & 1.32 & 1.34 & 1.30 & 1.32 & 2.05 & 1.35 & 1.49 \\
\hline $\mathrm{H} 2 \mathrm{~b}$ & 1.14 & 1.18 & 1.07 & 1.17 & 1.18 & 0.79 & 1.21 & 1.22 \\
\hline H $3 a$ & 1.47 & 1.63 & 1.61 & 2.12 & 1.64 & 1.77 & 1.71 & 1.86 \\
\hline $\mathrm{H} 3 \mathrm{~b}$ & 1.38 & 1.25 & 0.96 & 1.00 & 1.22 & 0.99 & 1.27 & 0.98 \\
\hline H 5 & 2.03 & 1.89 & 1.71 & 1.31 & 2.10 & 1.71 & 1.44 & 1.49 \\
\hline $\mathrm{H} 7 \mathrm{a}$ & 2.19 & 2.26 & 2.17 & 2.21 & 2.27 & 2.25 & 2.20 & 2.12 \\
\hline $\mathrm{H} 7 \mathrm{~b}$ & 2.15 & 2.16 & 2.02 & 2.15 & 2.24 & 2.19 & 2.09 & 2.09 \\
\hline H 8a & 1.91 & 1.81 & 1.95 & 1.93 & 2.07 & 1.85 & 2.14 & 1.93 \\
\hline $\mathrm{H} 8 \mathrm{~b}$ & 1.81 & 2.07 & 1.81 & 1.81 & 1.57 & 1.76 & 1.86 & 1.82 \\
\hline H 10 & 1.31 & 1.27 & 1.87 & 1.42 & 1.03 & 1.23 & 1.11 & 1.26 \\
\hline H 11 & 1.55 & 1.42 & 1.86 & 1.44 & 1.47 & 1.81 & 1.67 & 1.75 \\
\hline H $12 a-c$ & 0.93 & 1.04 & 0.99 & 1.12 & 0.81 & 0.92 & 0.97 & 0.91 \\
\hline H 13a & 1.63 & 1.93 & 2.09 & 1.94 & 1.46 & 1.44 & 1.66 & 1.44 \\
\hline $\mathrm{H} 13 \mathrm{~b}$ & 1.44 & 1.47 & 1.49 & 1.40 & 1.44 & 1.42 & 1.39 & 1.41 \\
\hline H $14 a$ & 1.53 & 1.59 & 1.58 & 1.66 & 1.39 & 1.44 & 1.46 & 1.46 \\
\hline $\mathrm{H} 14 \mathrm{~b}$ & 1.38 & 1.21 & 1.19 & 1.18 & 1.37 & 1.43 & 1.42 & 1.44 \\
\hline H 15 & 3.45 & 3.59 & 3.68 & 3.49 & 3.59 & 3.37 & 3.54 & 3.40 \\
\hline H 17a-c & 1.11 & 1.04 & 1.11 & 1.11 & 1.16 & 1.11 & 1.13 & 1.14 \\
\hline H 18a-c & 1.06 & 1.14 & 1.05 & 1.08 & 1.02 & 1.05 & 1.02 & 1.07 \\
\hline H 19a-c & 0.90 & 0.92 & 0.97 & 1.03 & 0.94 & 0.83 & 1.01 & 1.04 \\
\hline $\mathrm{H} 20 \mathrm{a}$ & 5.20 & 5.24 & 5.24 & 5.16 & 5.24 & 5.11 & 5.24 & 5.10 \\
\hline $\mathrm{H} 20 \mathrm{~b}$ & 4.95 & 4.88 & 5.01 & 4.98 & 5.11 & 4.83 & 5.07 & 4.87 \\
\hline
\end{tabular}




\begin{tabular}{|c|c|c|c|c|c|c|c|c|}
\hline \multicolumn{9}{|c|}{ Unscaled chemical shifts constants } \\
\hline Nuclei & Isomer 9 & Isomer 10 & Isomer 11 & Isomer 12 & Isomer 13 & Isomer 14 & Isomer 15 & Isomer 16 \\
\hline C 1 & 36.26 & 32.14 & 34.31 & 32.41 & 34.18 & 32.96 & 33.77 & 33.19 \\
\hline $\mathrm{C} 2$ & 28.63 & 27.67 & 27.20 & 29.58 & 29.44 & 27.97 & 30.97 & 30.38 \\
\hline C 3 & 24.78 & 33.02 & 18.41 & 33.94 & 29.87 & 32.09 & 27.48 & 33.39 \\
\hline $\mathrm{C} 4$ & 77.68 & 79.09 & 78.53 & 78.86 & 77.72 & 77.86 & 78.29 & 77.50 \\
\hline C 5 & 34.39 & 38.00 & 36.98 & 42.56 & 34.61 & 35.84 & 38.31 & 40.82 \\
\hline C 6 & 155.27 & 154.52 & 155.99 & 153.80 & 157.08 & 156.20 & 155.92 & 155.01 \\
\hline$C 7$ & 31.18 & 30.54 & 32.08 & 30.73 & 30.60 & 31.07 & 31.20 & 30.17 \\
\hline C 8 & 32.80 & 36.87 & 34.89 & 34.19 & 35.50 & 36.26 & 33.70 & 34.07 \\
\hline C 9 & 42.97 & 40.50 & 42.57 & 38.92 & 42.57 & 42.92 & 42.02 & 41.66 \\
\hline C 10 & 40.35 & 28.35 & 39.51 & 28.86 & 36.30 & 32.44 & 35.14 & 31.67 \\
\hline C 11 & 44.17 & 44.74 & 45.58 & 44.24 & 37.56 & 37.12 & 36.49 & 37.62 \\
\hline C 12 & 18.59 & 19.59 & 18.96 & 19.39 & 16.82 & 16.21 & 17.18 & 16.39 \\
\hline C 13 & 30.37 & 31.60 & 30.52 & 30.71 & 29.71 & 29.01 & 30.04 & 28.90 \\
\hline C 14 & 28.14 & 29.33 & 28.73 & 29.28 & 22.65 & 22.34 & 22.97 & 22.13 \\
\hline C 15 & 76.74 & 77.03 & 76.64 & 76.88 & 77.41 & 77.47 & 77.37 & 76.91 \\
\hline C 16 & 73.90 & 73.30 & 74.36 & 73.41 & 74.14 & 74.33 & 73.95 & 73.82 \\
\hline C 17 & 24.61 & 24.00 & 24.50 & 24.02 & 24.20 & 24.01 & 24.15 & 23.86 \\
\hline C 18 & 28.47 & 26.99 & 27.95 & 27.07 & 27.45 & 27.04 & 27.17 & 26.91 \\
\hline C 19 & 19.82 & 19.53 & 19.17 & 22.67 & 19.52 & 18.86 & 20.51 & 21.28 \\
\hline C 20 & 99.25 & 99.66 & 99.86 & 99.87 & 99.05 & 99.36 & 99.20 & 99.29 \\
\hline H 1 & 1.87 & 2.07 & 1.99 & 2.00 & 1.94 & 1.99 & 2.04 & 2.06 \\
\hline $\mathrm{H} 2 \mathrm{a}$ & 1.22 & 2.18 & 1.49 & 1.61 & 1.40 & 1.85 & 1.56 & 1.48 \\
\hline $\mathrm{H} 2 \mathrm{~b}$ & 0.96 & 0.87 & 1.05 & 1.42 & 1.03 & 0.86 & 1.05 & 1.28 \\
\hline H 3a & 1.86 & 1.81 & 1.63 & 1.74 & 1.82 & 1.40 & 1.66 & 1.48 \\
\hline $\mathrm{H} 3 \mathrm{~b}$ & 1.02 & 1.07 & 1.21 & 1.14 & 1.37 & 1.25 & 1.54 & 1.15 \\
\hline H 5 & 2.15 & 1.66 & 2.17 & 1.51 & 2.01 & 1.39 & 1.86 & 1.23 \\
\hline $\mathrm{H} 7 \mathrm{a}$ & 2.20 & 2.21 & 2.19 & 2.25 & 2.18 & 2.29 & 2.15 & 2.17 \\
\hline $\mathrm{H} 7 \mathrm{~b}$ & 2.10 & 2.16 & 2.13 & 2.16 & 2.16 & 2.24 & 2.08 & 2.03 \\
\hline H 8a & 2.00 & 1.72 & 1.72 & 1.82 & 1.91 & 1.91 & 1.84 & 1.95 \\
\hline $\mathrm{H} 8 \mathrm{~b}$ & 1.68 & 1.66 & 1.83 & 1.74 & 1.66 & 1.79 & 1.80 & 1.73 \\
\hline H 10 & 1.25 & 1.84 & 1.38 & 1.72 & 1.56 & 2.01 & 1.59 & 2.04 \\
\hline H 11 & 1.59 & 1.52 & 1.57 & 1.47 & 1.68 & 1.64 & 1.76 & 1.68 \\
\hline H 12a-c & 0.87 & 0.95 & 0.91 & 0.89 & 1.17 & 0.95 & 1.18 & 0.92 \\
\hline H 13a & 1.53 & 1.50 & 1.47 & 1.57 & 1.99 & 2.12 & 1.97 & 2.11 \\
\hline $\mathrm{H} 13 \mathrm{~b}$ & 1.37 & 1.49 & 1.40 & 1.52 & 1.41 & 1.43 & 1.42 & 1.45 \\
\hline $\mathrm{H} 14 \mathrm{a}$ & 1.54 & 1.47 & 1.49 & 1.51 & 1.68 & 1.60 & 1.66 & 1.63 \\
\hline $\mathrm{H} 14 \mathrm{~b}$ & 1.44 & 1.46 & 1.42 & 1.49 & 1.14 & 1.18 & 1.13 & 1.20 \\
\hline H 15 & 3.32 & 3.67 & 3.42 & 3.64 & 3.46 & 3.72 & 3.46 & 3.63 \\
\hline H 17a-c & 1.01 & 1.07 & 1.06 & 1.04 & 1.04 & 1.09 & 1.06 & 1.07 \\
\hline H 18a-c & 1.14 & 1.15 & 1.13 & 1.14 & 1.10 & 1.13 & 1.09 & 1.12 \\
\hline H 19a-c & 0.89 & 0.81 & 0.99 & 0.94 & 0.88 & 0.84 & 1.01 & 1.00 \\
\hline $\mathrm{H} 20 \mathrm{a}$ & 5.17 & 5.17 & 5.24 & 5.16 & 5.22 & 5.07 & 5.21 & 5.15 \\
\hline $\mathrm{H} 20 \mathrm{~b}$ & 5.02 & 4.93 & 5.04 & 4.97 & 4.95 & 4.87 & 4.96 & 5.05 \\
\hline
\end{tabular}




\begin{tabular}{|c|c|c|c|c|c|c|c|c|}
\hline \multicolumn{9}{|c|}{ Scaled chemical shifts constants } \\
\hline Nuclei & Isomer 1 & Isomer 2 & Isomer 3 & Isomer 4 & Isomer 5 & Isomer 6 & Isomer 7 & Isomer 8 \\
\hline C 1 & 32.91 & 32.92 & 32.30 & 32.40 & 32.10 & 29.84 & 31.57 & 31.05 \\
\hline $\mathrm{C} 2$ & 26.32 & 27.34 & 30.28 & 28.11 & 27.08 & 25.09 & 31.87 & 26.79 \\
\hline C 3 & 26.42 & 32.90 & 29.39 & 26.22 & 34.50 & 16.50 & 36.54 & 17.95 \\
\hline $\mathrm{C} 4$ & 76.35 & 75.98 & 77.52 & 77.91 & 76.71 & 78.69 & 77.44 & 78.71 \\
\hline $\mathrm{C} 5$ & 33.06 & 33.46 & 37.52 & 37.45 & 33.32 & 34.36 & 38.82 & 38.47 \\
\hline C 6 & 155.88 & 156.07 & 155.37 & 154.98 & 156.11 & 154.98 & 156.80 & 155.47 \\
\hline C 7 & 28.56 & 28.67 & 28.13 & 28.00 & 29.06 & 30.31 & 27.16 & 29.24 \\
\hline C 8 & 31.27 & 32.18 & 31.68 & 31.76 & 29.72 & 34.99 & 26.58 & 31.93 \\
\hline C 9 & 40.69 & 42.15 & 40.74 & 40.46 & 41.02 & 38.54 & 41.58 & 36.06 \\
\hline C 10 & 34.79 & 32.69 & 31.39 & 35.80 & 26.90 & 37.02 & 25.45 & 36.65 \\
\hline C 11 & 40.83 & 37.64 & 34.45 & 36.08 & 39.79 & 41.28 & 42.17 & 41.48 \\
\hline C 12 & 15.02 & 13.40 & 13.89 & 13.69 & 15.54 & 16.31 & 14.45 & 15.66 \\
\hline C 13 & 27.83 & 27.48 & 27.59 & 27.31 & 27.05 & 29.33 & 27.51 & 28.44 \\
\hline C 14 & 23.90 & 20.02 & 20.61 & 20.81 & 25.99 & 26.88 & 23.24 & 26.38 \\
\hline C 15 & 75.64 & 76.35 & 77.21 & 76.26 & 75.49 & 75.45 & 75.27 & 75.42 \\
\hline C 16 & 72.32 & 72.47 & 72.55 & 72.66 & 73.08 & 72.06 & 70.90 & 71.95 \\
\hline C 17 & 24.12 & 22.38 & 24.79 & 24.95 & 24.26 & 25.18 & 23.17 & 24.58 \\
\hline C 18 & 22.97 & 24.61 & 21.45 & 21.98 & 20.98 & 21.91 & 19.75 & 21.14 \\
\hline C 19 & 16.93 & 17.03 & 18.20 & 18.30 & 16.32 & 17.21 & 15.34 & 18.78 \\
\hline C 20 & 98.51 & 98.57 & 99.26 & 99.18 & 99.29 & 98.36 & 98.68 & 98.14 \\
\hline H 1 & 1.81 & 1.80 & 1.86 & 1.81 & 1.79 & 2.02 & 1.56 & 1.92 \\
\hline $\mathrm{H} 2 \mathrm{a}$ & 1.20 & 1.24 & 1.23 & 1.22 & 1.29 & 1.95 & 1.29 & 1.42 \\
\hline $\mathrm{H} 2 \mathrm{~b}$ & 1.08 & 1.10 & 0.98 & 1.10 & 1.16 & 0.75 & 1.16 & 1.17 \\
\hline H $3 a$ & 1.40 & 1.52 & 1.49 & 2.00 & 1.57 & 1.69 & 1.63 & 1.78 \\
\hline $\mathrm{H} 3 \mathrm{~b}$ & 1.31 & 1.17 & 0.88 & 0.94 & 1.20 & 0.94 & 1.22 & 0.93 \\
\hline H 5 & 1.93 & 1.77 & 1.58 & 1.24 & 1.99 & 1.63 & 1.38 & 1.43 \\
\hline H 7a & 2.08 & 2.12 & 2.01 & 2.09 & 2.14 & 2.14 & 2.09 & 2.03 \\
\hline $\mathrm{H} 7 \mathrm{~b}$ & 2.04 & 2.02 & 1.87 & 2.02 & 2.12 & 2.09 & 1.98 & 2.00 \\
\hline H 8a & 1.81 & 1.70 & 1.81 & 1.82 & 1.96 & 1.76 & 2.02 & 1.85 \\
\hline $\mathrm{H} 8 \mathrm{~b}$ & 1.71 & 1.94 & 1.67 & 1.70 & 1.51 & 1.68 & 1.77 & 1.74 \\
\hline H 10 & 1.24 & 1.19 & 1.73 & 1.33 & 1.03 & 1.17 & 1.08 & 1.20 \\
\hline H 11 & 1.47 & 1.33 & 1.72 & 1.36 & 1.42 & 1.73 & 1.59 & 1.68 \\
\hline H $12 a-c$ & 0.89 & 0.97 & 0.91 & 1.06 & 0.83 & 0.88 & 0.94 & 0.86 \\
\hline H 13a & 1.54 & 1.81 & 1.94 & 1.83 & 1.41 & 1.37 & 1.58 & 1.37 \\
\hline $\mathrm{H} 13 \mathrm{~b}$ & 1.37 & 1.37 & 1.38 & 1.32 & 1.39 & 1.35 & 1.33 & 1.35 \\
\hline H 14a & 1.46 & 1.48 & 1.46 & 1.57 & 1.35 & 1.37 & 1.40 & 1.39 \\
\hline $\mathrm{H} 14 \mathrm{~b}$ & 1.32 & 1.13 & 1.09 & 1.11 & 1.33 & 1.37 & 1.36 & 1.37 \\
\hline H 15 & 3.26 & 3.36 & 3.42 & 3.29 & 3.32 & 3.21 & 3.33 & 3.27 \\
\hline H 17a-c & 1.06 & 0.97 & 1.02 & 1.04 & 1.15 & 1.06 & 1.09 & 1.08 \\
\hline H 18a-c & 1.01 & 1.07 & 0.97 & 1.02 & 1.02 & 1.01 & 0.99 & 1.02 \\
\hline H 19a-c & 0.86 & 0.85 & 0.90 & 0.97 & 0.95 & 0.80 & 0.98 & 0.99 \\
\hline $\mathrm{H} 20 \mathrm{a}$ & 4.91 & 4.92 & 4.87 & 4.88 & 4.81 & 4.86 & 4.90 & 4.91 \\
\hline $\mathrm{H} 20 \mathrm{~b}$ & 4.67 & 4.58 & 4.66 & 4.71 & 4.69 & 4.60 & 4.74 & 4.69 \\
\hline
\end{tabular}




\begin{tabular}{|c|c|c|c|c|c|c|c|c|}
\hline \multicolumn{9}{|c|}{ Scaled chemical shifts constants } \\
\hline Nuclei & Isomer 9 & Isomer 10 & Isomer 11 & Isomer 12 & Isomer 13 & Isomer 14 & Isomer 15 & Isomer 16 \\
\hline C 1 & 33.78 & 29.55 & 32.03 & 29.42 & 32.46 & 31.48 & 31.99 & 31.33 \\
\hline C 2 & 25.97 & 24.94 & 24.84 & 26.47 & 27.68 & 26.44 & 29.15 & 28.44 \\
\hline C 3 & 22.02 & 30.47 & 15.94 & 31.02 & 28.11 & 30.60 & 25.61 & 31.53 \\
\hline C 4 & 76.21 & 77.98 & 76.78 & 77.82 & 76.39 & 76.80 & 77.20 & 76.72 \\
\hline C 5 & 31.87 & 35.60 & 34.74 & 40.00 & 32.89 & 34.39 & 36.60 & 39.14 \\
\hline C 6 & 155.71 & 155.76 & 155.18 & 155.91 & 156.45 & 155.88 & 156.01 & 156.13 \\
\hline$C 7$ & 28.58 & 27.91 & 29.77 & 27.67 & 28.85 & 29.57 & 29.39 & 28.23 \\
\hline C 8 & 30.24 & 34.43 & 32.62 & 31.27 & 33.80 & 34.81 & 31.92 & 32.22 \\
\hline C 9 & 40.66 & 38.18 & 40.39 & 36.20 & 40.93 & 41.53 & 40.37 & 40.00 \\
\hline C 10 & 37.97 & 25.64 & 37.30 & 25.71 & 34.60 & 30.95 & 33.38 & 29.76 \\
\hline C 11 & 41.89 & 42.55 & 43.43 & 41.74 & 35.88 & 35.68 & 34.76 & 35.86 \\
\hline C 12 & 15.69 & 16.62 & 16.50 & 15.85 & 14.95 & 14.58 & 15.15 & 14.11 \\
\hline C 13 & 27.75 & 29.00 & 28.20 & 27.65 & 27.95 & 27.50 & 28.20 & 26.93 \\
\hline C 14 & 25.46 & 26.66 & 26.39 & 26.16 & 20.83 & 20.75 & 21.03 & 20.00 \\
\hline C 15 & 75.26 & 75.85 & 74.87 & 75.76 & 76.08 & 76.41 & 76.26 & 76.12 \\
\hline C 16 & 72.34 & 72.00 & 72.56 & 72.14 & 72.78 & 73.24 & 72.78 & 72.95 \\
\hline C 17 & 21.85 & 21.17 & 22.11 & 20.68 & 22.40 & 22.45 & 22.23 & 21.76 \\
\hline C 18 & 25.81 & 24.25 & 25.59 & 23.85 & 25.67 & 25.50 & 25.30 & 24.89 \\
\hline C 19 & 16.94 & 16.55 & 16.70 & 19.27 & 17.67 & 17.25 & 18.53 & 19.12 \\
\hline C 20 & 98.31 & 99.18 & 98.38 & 99.72 & 97.91 & 98.51 & 98.43 & 99.05 \\
\hline H 1 & 1.79 & 1.93 & 1.87 & 1.86 & 1.80 & 1.85 & 1.88 & 1.91 \\
\hline $\mathrm{H} 2 \mathrm{a}$ & 1.19 & 2.03 & 1.41 & 1.49 & 1.28 & 1.71 & 1.42 & 1.36 \\
\hline $\mathrm{H} 2 \mathrm{~b}$ & 0.95 & 0.80 & 1.01 & 1.31 & 0.93 & 0.75 & 0.92 & 1.17 \\
\hline H 3a & 1.78 & 1.69 & 1.54 & 1.62 & 1.68 & 1.28 & 1.51 & 1.36 \\
\hline $\mathrm{H} 3 \mathrm{~b}$ & 1.01 & 0.98 & 1.15 & 1.04 & 1.25 & 1.13 & 1.39 & 1.04 \\
\hline H 5 & 2.05 & 1.54 & 2.04 & 1.39 & 1.86 & 1.27 & 1.71 & 1.12 \\
\hline $\mathrm{H} 7 \mathrm{a}$ & 2.09 & 2.06 & 2.06 & 2.10 & 2.02 & 2.14 & 1.99 & 2.02 \\
\hline $\mathrm{H} 7 \mathrm{~b}$ & 2.01 & 2.02 & 2.01 & 2.02 & 2.01 & 2.09 & 1.92 & 1.89 \\
\hline H 8a & 1.91 & 1.60 & 1.63 & 1.70 & 1.77 & 1.77 & 1.69 & 1.81 \\
\hline $\mathrm{H} 8 \mathrm{~b}$ & 1.61 & 1.54 & 1.72 & 1.62 & 1.53 & 1.66 & 1.64 & 1.60 \\
\hline H 10 & 1.23 & 1.71 & 1.31 & 1.60 & 1.43 & 1.87 & 1.45 & 1.90 \\
\hline H 11 & 1.54 & 1.41 & 1.49 & 1.36 & 1.54 & 1.52 & 1.61 & 1.55 \\
\hline H 12a-c & 0.88 & 0.88 & 0.88 & 0.81 & 1.06 & 0.84 & 1.05 & 0.82 \\
\hline $\mathrm{H} 13 \mathrm{a}$ & 1.48 & 1.39 & 1.40 & 1.45 & 1.84 & 1.98 & 1.81 & 1.96 \\
\hline $\mathrm{H} 13 \mathrm{~b}$ & 1.34 & 1.38 & 1.33 & 1.41 & 1.29 & 1.31 & 1.28 & 1.33 \\
\hline H 14a & 1.49 & 1.36 & 1.42 & 1.40 & 1.55 & 1.47 & 1.51 & 1.51 \\
\hline $\mathrm{H} 14 \mathrm{~b}$ & 1.40 & 1.36 & 1.35 & 1.38 & 1.03 & 1.07 & 1.00 & 1.09 \\
\hline H 15 & 3.13 & 3.44 & 3.20 & 3.43 & 3.24 & 3.52 & 3.25 & 3.43 \\
\hline H 17a-c & 1.01 & 0.99 & 1.01 & 0.95 & 0.94 & 0.98 & 0.93 & 0.97 \\
\hline H 18a-c & 1.12 & 1.07 & 1.08 & 1.05 & 1.00 & 1.02 & 0.96 & 1.01 \\
\hline H 19a-c & 0.89 & 0.75 & 0.95 & 0.85 & 0.79 & 0.74 & 0.89 & 0.90 \\
\hline $\mathrm{H} 20 \mathrm{a}$ & 4.83 & 4.85 & 4.88 & 4.89 & 4.91 & 4.83 & 4.94 & 4.89 \\
\hline $\mathrm{H} 20 \mathrm{~b}$ & 4.69 & 4.63 & 4.70 & 4.71 & 4.65 & 4.64 & 4.70 & 4.79 \\
\hline
\end{tabular}


Absolute unscaled error

\begin{tabular}{|c|c|c|c|c|c|c|c|c|c|c|c|c|c|c|c|c|}
\hline & Isomer 1 & Isomer 2 & Isomer 3 & Isomer 4 & Isomer 5 & Isomer 6 & Isomer 7 & Isomer 8 & Isomer 9 & Isomer 10 & Isomer 11 & Isomer 12 & Isomer 13 & Isomer 14 & Isomer 15 & Isomer 16 \\
\hline C 1 & 3.8 & 3.6 & 3.0 & 2.7 & 3.4 & 0.3 & 4.1 & 2.0 & 4.7 & 0.5 & 2.7 & 0.8 & 2.6 & 1.4 & 2.2 & 1.6 \\
\hline C 2 & 3.5 & 4.2 & 7.1 & 4.6 & 4.6 & 1.7 & 10.5 & 3.9 & 3.1 & 2.2 & 1.7 & 4.1 & 3.9 & 2.5 & 5.5 & 4.9 \\
\hline C 3 & 6.7 & 12.9 & 9.4 & 5.9 & 15.0 & 3.6 & 18.2 & 1.5 & 2.5 & 10.7 & 3.9 & 11.6 & 7.6 & 9.8 & 5.2 & 11.1 \\
\hline C 4 & 3.4 & 3.1 & 4.1 & 4.3 & 3.8 & 5.6 & 5.1 & 5.6 & 3.2 & 4.6 & 4.0 & 4.4 & 3.2 & 3.4 & 3.8 & 3.0 \\
\hline C5 & 6.2 & 6.4 & 10.4 & 9.9 & 6.9 & 7.1 & 13.3 & 11.5 & 5.1 & 8.7 & 7.7 & 13.3 & 5.3 & 6.5 & 9.0 & 11.5 \\
\hline C 6 & 0.6 & 1.4 & 0.5 & 0.8 & 0.3 & 0.3 & 0.4 & 0.1 & 0.3 & 0.5 & 1.0 & 1.2 & 2.1 & 1.2 & 0.9 & 0.0 \\
\hline C 7 & 1.9 & 1.8 & 1.3 & 0.8 & 2.8 & 3.2 & 2.3 & 2.6 & 2.0 & 1.3 & 2.9 & 1.5 & 1.4 & 1.9 & 2.0 & 1.0 \\
\hline C 8 & 3.6 & 4.3 & 3.8 & 3.5 & 2.5 & 6.8 & 0.7 & 4.3 & 2.6 & 6.7 & 4.7 & 4.0 & 5.3 & 6.1 & 3.5 & 3.9 \\
\hline C 9 & 4.8 & 6.1 & 4.6 & 4.0 & 5.4 & 2.3 & 7.1 & 0.3 & 4.8 & 2.3 & 4.4 & 0.7 & 4.4 & 4.7 & 3.8 & 3.5 \\
\hline C 10 & 1.3 & 0.9 & 2.2 & 1.7 & 6.0 & 3.1 & 6.0 & 3.2 & 4.4 & 7.6 & 3.6 & 7.0 & 0.4 & 3.5 & 0.8 & 4.2 \\
\hline C 11 & 1.5 & 1.8 & 4.9 & 3.7 & 0.8 & 1.6 & 4.2 & 2.2 & 2.6 & 3.1 & 4.0 & 2.6 & 4.0 & 4.5 & 5.1 & 4.0 \\
\hline C 12 & 0.6 & 1.3 & 0.7 & 1.3 & 1.6 & 1.3 & 2.0 & 1.3 & 1.3 & 2.3 & 1.7 & 2.1 & 0.5 & 1.1 & 0.1 & 0.9 \\
\hline C 13 & 2.4 & 1.9 & 2.0 & 1.3 & 2.1 & 3.4 & 3.8 & 3.1 & 2.4 & 3.6 & 2.5 & 2.7 & 1.7 & 1.0 & 2.0 & 0.9 \\
\hline C 14 & 0.8 & 3.3 & 2.6 & 2.9 & 3.2 & 3.2 & 1.9 & 3.2 & 2.3 & 3.5 & 2.9 & 3.5 & 3.1 & 3.5 & 2.8 & 3.7 \\
\hline C 15 & 1.9 & 2.6 & 3.0 & 1.9 & 1.8 & 1.6 & 2.2 & 1.6 & 1.4 & 1.7 & 1.3 & 1.6 & 2.1 & 2.2 & 2.1 & 1.6 \\
\hline C 16 & 1.8 & 2.0 & 1.7 & 1.6 & 2.6 & 1.4 & 1.2 & 1.4 & 1.8 & 1.2 & 2.3 & 1.3 & 2.0 & 2.2 & 1.8 & 1.7 \\
\hline C 17 & 2.5 & 0.5 & 3.0 & 2.7 & 3.1 & 3.0 & 3.4 & 3.0 & 0.3 & 0.3 & 0.2 & 0.3 & 0.1 & 0.3 & 0.1 & 0.4 \\
\hline C 18 & 1.5 & 0.2 & 3.2 & 3.1 & 3.0 & 3.1 & 2.8 & 3.3 & 1.3 & 0.2 & 0.7 & 0.1 & 0.2 & 0.2 & 0.0 & 0.3 \\
\hline C 19 & 0.9 & 0.7 & 1.9 & 1.6 & 0.8 & 0.6 & 1.3 & 2.7 & 0.9 & 0.6 & 0.3 & 3.8 & 0.6 & 0.0 & 1.6 & 2.4 \\
\hline C 20 & 2.6 & 2.3 & 2.3 & 2.5 & 1.9 & 2.6 & 2.2 & 3.1 & 2.9 & 2.4 & 2.2 & 2.2 & 3.1 & 2.7 & 2.9 & 2.8 \\
\hline Average & 2.6 & 3.1 & 3.6 & 3.0 & 3.6 & 2.8 & 4.6 & 3.0 & 2.5 & 3.2 & 2.7 & 3.4 & 2.7 & 2.9 & 2.8 & 3.2 \\
\hline H 1 & 0.14 & 0.15 & 0.24 & 0.15 & 0.11 & 0.35 & 0.13 & 0.23 & 0.10 & 0.30 & 0.22 & 0.23 & 0.17 & 0.22 & 0.27 & 0.29 \\
\hline $\mathrm{H} 2 \mathrm{a}$ & 0.09 & 0.04 & 0.02 & 0.06 & 0.04 & 0.69 & 0.01 & 0.13 & 0.14 & 0.82 & 0.13 & 0.25 & 0.04 & 0.49 & 0.20 & 0.12 \\
\hline $\mathrm{H} 2 \mathrm{~b}$ & 0.38 & 0.42 & 0.31 & 0.41 & 0.42 & 0.03 & 0.45 & 0.46 & 0.20 & 0.11 & 0.29 & 0.66 & 0.27 & 0.10 & 0.29 & 0.52 \\
\hline H 3a & 0.37 & 0.21 & 0.23 & 0.28 & 0.20 & 0.07 & 0.13 & 0.02 & 0.02 & 0.03 & 0.21 & 0.10 & 0.02 & 0.44 & 0.18 & 0.36 \\
\hline H 3b & 0.41 & 0.28 & 0.01 & 0.03 & 0.25 & 0.02 & 0.30 & 0.01 & 0.05 & 0.10 & 0.24 & 0.17 & 0.40 & 0.28 & 0.57 & 0.18 \\
\hline H 5 & 0.03 & 0.11 & 0.29 & 0.69 & 0.10 & 0.29 & 0.56 & 0.51 & 0.15 & 0.34 & 0.17 & 0.49 & 0.01 & 0.61 & 0.14 & 0.77 \\
\hline $\mathrm{H} 7 \mathrm{a}$ & 0.02 & 0.09 & 0.00 & 0.04 & 0.10 & 0.08 & 0.03 & 0.05 & 0.03 & 0.04 & 0.02 & 0.08 & 0.01 & 0.12 & 0.02 & 0.00 \\
\hline $\mathrm{H} 7 \mathrm{~b}$ & 0.15 & 0.16 & 0.02 & 0.15 & 0.24 & 0.19 & 0.09 & 0.09 & 0.10 & 0.16 & 0.13 & 0.16 & 0.16 & 0.24 & 0.08 & 0.03 \\
\hline H $8 \mathrm{a}$ & 0.33 & 0.23 & 0.37 & 0.35 & 0.49 & 0.27 & 0.56 & 0.35 & 0.42 & 0.14 & 0.14 & 0.24 & 0.33 & 0.33 & 0.26 & 0.37 \\
\hline $\mathrm{H} 8 \mathrm{~b}$ & 0.23 & 0.49 & 0.23 & 0.23 & 0.01 & 0.18 & 0.28 & 0.24 & 0.10 & 0.08 & 0.25 & 0.16 & 0.08 & 0.21 & 0.22 & 0.15 \\
\hline H 10 & 0.21 & 0.17 & 0.77 & 0.32 & 0.07 & 0.13 & 0.01 & 0.16 & 0.15 & 0.74 & 0.28 & 0.62 & 0.46 & 0.91 & 0.49 & 0.94 \\
\hline H 11 & 0.06 & 0.19 & 0.25 & 0.17 & 0.14 & 0.20 & 0.06 & 0.14 & 0.02 & 0.09 & 0.04 & 0.14 & 0.07 & 0.03 & 0.15 & 0.07 \\
\hline H 12a-c & 0.04 & 0.15 & 0.10 & 0.23 & 0.08 & 0.03 & 0.08 & 0.02 & 0.02 & 0.06 & 0.02 & 0.00 & 0.28 & 0.06 & 0.29 & 0.03 \\
\hline H 13a & 0.04 & 0.34 & 0.50 & 0.35 & 0.13 & 0.15 & 0.07 & 0.15 & 0.06 & 0.09 & 0.12 & 0.02 & 0.40 & 0.53 & 0.38 & 0.52 \\
\hline H 13b & 0.08 & 0.11 & 0.13 & 0.04 & 0.08 & 0.06 & 0.03 & 0.05 & 0.01 & 0.13 & 0.04 & 0.16 & 0.05 & 0.07 & 0.06 & 0.09 \\
\hline H 14a & 0.01 & 0.05 & 0.04 & 0.12 & 0.15 & 0.10 & 0.08 & 0.08 & 0.00 & 0.07 & 0.05 & 0.03 & 0.14 & 0.06 & 0.12 & 0.09 \\
\hline H 14b & 0.00 & 0.17 & 0.19 & 0.20 & 0.01 & 0.05 & 0.04 & 0.06 & 0.06 & 0.08 & 0.04 & 0.11 & 0.24 & 0.20 & 0.25 & 0.18 \\
\hline H 15 & 0.25 & 0.39 & 0.48 & 0.29 & 0.39 & 0.17 & 0.34 & 0.20 & 0.12 & 0.47 & 0.22 & 0.44 & 0.26 & 0.52 & 0.26 & 0.43 \\
\hline H 17a-c & 0.02 & 0.09 & 0.02 & 0.02 & 0.03 & 0.02 & 0.00 & 0.01 & 0.12 & 0.06 & 0.07 & 0.09 & 0.09 & 0.04 & 0.07 & 0.06 \\
\hline H 18a-c & 0.14 & 0.06 & 0.15 & 0.12 & 0.18 & 0.15 & 0.18 & 0.13 & 0.06 & 0.05 & 0.07 & 0.06 & 0.10 & 0.07 & 0.11 & 0.08 \\
\hline H 19a-c & 0.05 & 0.03 & 0.02 & 0.08 & 0.01 & 0.12 & 0.06 & 0.09 & 0.06 & 0.14 & 0.04 & 0.01 & 0.07 & 0.11 & 0.06 & 0.05 \\
\hline H 20a & 0.37 & 0.41 & 0.41 & 0.33 & 0.41 & 0.28 & 0.41 & 0.27 & 0.34 & 0.34 & 0.41 & 0.33 & 0.39 & 0.24 & 0.38 & 0.32 \\
\hline $\mathrm{H} 20 \mathrm{~b}$ & 0.33 & 0.26 & 0.39 & 0.36 & 0.49 & 0.21 & 0.45 & 0.25 & 0.40 & 0.31 & 0.42 & 0.35 & 0.33 & 0.25 & 0.34 & 0.43 \\
\hline Average & 0.16 & 0.20 & 0.23 & 0.22 & 0.18 & 0.17 & 0.19 & 0.16 & 0.12 & 0.21 & 0.16 & 0.21 & 0.19 & 0.27 & 0.23 & 0.26 \\
\hline
\end{tabular}


Absolute scaled error

\begin{tabular}{|c|c|c|c|c|c|c|c|c|c|c|c|c|c|c|c|c|}
\hline & Isomer 1 & Isomer 2 & Isomer 3 & Isomer 4 & Isomer 5 & Isomer 6 & Isomer 7 & Isomer 8 & Isomer 9 & Isomer 10 & Isomer 11 & Isomer 12 & Isomer 13 & Isomer 14 & Isomer 15 & Isomer 16 \\
\hline C 1 & 1.3 & 1.3 & 0.7 & 0.8 & 0.5 & 1.8 & 0.0 & 0.6 & 2.2 & 2.0 & 0.4 & 2.2 & 0.9 & 0.1 & 0.4 & 0.3 \\
\hline$C 2$ & 0.8 & 1.8 & 4.8 & 2.6 & 1.6 & 0.4 & 6.4 & 1.3 & 0.5 & 0.6 & 0.7 & 1.0 & 2.2 & 0.9 & 3.6 & 2.9 \\
\hline C 3 & 4.1 & 10.6 & 7.1 & 3.9 & 12.2 & 5.8 & 14.2 & 4.3 & 0.3 & 8.2 & 6.4 & 8.7 & 5.8 & 8.3 & 3.3 & 9.2 \\
\hline C 4 & 1.8 & 1.5 & 3.0 & 3.4 & 2.2 & 4.2 & 2.9 & 4.2 & 1.7 & 3.5 & 2.3 & 3.3 & 1.9 & 2.3 & 2.7 & 2.2 \\
\hline C 5 & 3.8 & 4.2 & 8.2 & 8.2 & 4.0 & 5.1 & 9.5 & 9.2 & 2.6 & 6.3 & 5.4 & 10.7 & 3.6 & 5.1 & 7.3 & 9.8 \\
\hline C 6 & 0.9 & 1.1 & 0.4 & 0.0 & 1.1 & 0.0 & 1.8 & 0.5 & 0.7 & 0.8 & 0.2 & 0.9 & 1.5 & 0.9 & 1.0 & 1.1 \\
\hline C 7 & 0.6 & 0.5 & 1.1 & 1.2 & 0.1 & 1.1 & 2.0 & 0.0 & 0.6 & 1.3 & 0.6 & 1.5 & 0.3 & 0.4 & 0.2 & 1.0 \\
\hline C 8 & 1.1 & 2.0 & 1.5 & 1.6 & 0.5 & 4.8 & 3.6 & 1.7 & 0.0 & 4.2 & 2.4 & 1.1 & 3.6 & 4.6 & 1.7 & 2.0 \\
\hline C9 & 2.5 & 3.9 & 2.5 & 2.3 & 2.8 & 0.3 & 3.4 & 2.1 & 2.5 & 0.0 & 2.2 & 2.0 & 2.7 & 3.3 & 2.2 & 1.8 \\
\hline C 10 & 1.1 & 3.2 & 4.5 & 0.1 & 9.0 & 1.1 & 10.4 & 0.7 & 2.1 & 10.3 & 1.4 & 10.2 & 1.3 & 4.9 & 2.5 & 6.1 \\
\hline C 11 & 0.8 & 4.0 & 7.2 & 5.5 & 1.8 & 0.3 & 0.6 & 0.1 & 0.3 & 0.9 & 1.8 & 0.1 & 5.7 & 5.9 & 6.8 & 5.7 \\
\hline C 12 & 2.3 & 3.9 & 3.4 & 3.6 & 1.8 & 1.0 & 2.8 & 1.6 & 1.6 & 0.7 & 0.8 & 1.4 & 2.3 & 2.7 & 2.1 & 3.2 \\
\hline C 13 & 0.2 & 0.5 & 0.4 & 0.7 & 0.9 & 1.3 & 0.5 & 0.4 & 0.3 & 1.0 & 0.2 & 0.4 & 0.0 & 0.5 & 0.2 & 1.1 \\
\hline C 14 & 1.9 & 5.8 & 5.2 & 5.0 & 0.2 & 1.1 & 2.6 & 0.6 & 0.3 & 0.9 & 0.6 & 0.4 & 5.0 & 5.0 & 4.8 & 5.8 \\
\hline C 15 & 0.3 & 1.1 & 1.9 & 1.0 & 0.2 & 0.2 & 0.0 & 0.1 & 0.0 & 0.6 & 0.4 & 0.5 & 0.8 & 1.1 & 1.0 & 0.8 \\
\hline C 16 & 0.2 & 0.4 & 0.5 & 0.6 & 1.0 & 0.0 & 1.2 & 0.2 & 0.2 & 0.1 & 0.5 & 0.0 & 0.7 & 1.1 & 0.7 & 0.8 \\
\hline C 17 & 0.2 & 1.9 & 0.5 & 0.6 & 0.0 & 0.9 & 1.1 & 0.3 & 2.4 & 3.1 & 2.2 & 3.6 & 1.9 & 1.9 & 2.1 & 2.5 \\
\hline C 18 & 4.2 & 2.6 & 5.8 & 5.2 & 6.2 & 5.3 & 7.4 & 6.1 & 1.4 & 3.0 & 1.6 & 3.3 & 1.5 & 1.7 & 1.9 & 2.3 \\
\hline C 19 & 2.0 & 1.9 & 0.7 & 0.6 & 2.6 & 1.7 & 3.6 & 0.1 & 2.0 & 2.4 & 2.2 & 0.4 & 1.2 & 1.7 & 0.4 & 0.2 \\
\hline C 20 & 3.6 & 3.5 & 2.8 & 2.9 & 2.8 & 3.7 & 3.4 & 4.0 & 3.8 & 2.9 & 3.7 & 2.4 & 4.2 & 3.6 & 3.7 & 3.1 \\
\hline Average & 1.7 & 2.8 & 3.1 & 2.5 & 2.6 & 2.0 & 3.9 & 1.9 & 1.3 & 2.6 & 1.8 & 2.7 & 2.4 & 2.8 & 2.4 & 3.1 \\
\hline H 1 & 0.04 & 0.03 & 0.09 & 0.04 & 0.02 & 0.25 & 0.21 & 0.15 & 0.02 & 0.16 & 0.10 & 0.09 & 0.03 & 0.08 & 0.11 & 0.14 \\
\hline $\mathrm{H} 2 \mathrm{a}$ & 0.16 & 0.12 & 0.13 & 0.14 & 0.07 & 0.59 & 0.07 & 0.06 & 0.17 & 0.67 & 0.05 & 0.13 & 0.08 & 0.35 & 0.06 & 0.00 \\
\hline $\mathrm{H} 2 \mathrm{~b}$ & 0.32 & 0.34 & 0.22 & 0.34 & 0.40 & 0.01 & 0.40 & 0.41 & 0.19 & 0.04 & 0.25 & 0.55 & 0.17 & 0.01 & 0.16 & 0.41 \\
\hline H $3 a$ & 0.44 & 0.32 & 0.35 & 0.16 & 0.27 & 0.15 & 0.21 & 0.06 & 0.06 & 0.15 & 0.30 & 0.22 & 0.16 & 0.56 & 0.33 & 0.48 \\
\hline H $3 b$ & 0.34 & 0.20 & 0.09 & 0.03 & 0.23 & 0.03 & 0.25 & 0.04 & 0.04 & 0.01 & 0.18 & 0.07 & 0.28 & 0.16 & 0.42 & 0.07 \\
\hline H 5 & 0.07 & 0.23 & 0.42 & 0.76 & 0.01 & 0.37 & 0.62 & 0.57 & 0.05 & 0.46 & 0.04 & 0.61 & 0.14 & 0.73 & 0.29 & 0.88 \\
\hline $\mathrm{H} 7 \mathrm{a}$ & 0.09 & 0.05 & 0.16 & 0.08 & 0.03 & 0.03 & 0.08 & 0.14 & 0.08 & 0.11 & 0.11 & 0.07 & 0.15 & 0.03 & 0.18 & 0.15 \\
\hline$H 7 b$ & 0.04 & 0.02 & 0.13 & 0.02 & 0.12 & 0.09 & 0.02 & 0.00 & 0.01 & 0.02 & 0.01 & 0.02 & 0.01 & 0.09 & 0.08 & 0.11 \\
\hline $\mathrm{H} 8 \mathrm{a}$ & 0.23 & 0.12 & 0.23 & 0.24 & 0.38 & 0.18 & 0.44 & 0.27 & 0.33 & 0.02 & 0.05 & 0.12 & 0.19 & 0.19 & 0.11 & 0.23 \\
\hline$H 8 b$ & 0.13 & 0.36 & 0.09 & 0.12 & 0.07 & 0.10 & 0.19 & 0.16 & 0.03 & 0.04 & 0.14 & 0.04 & 0.05 & 0.08 & 0.06 & 0.02 \\
\hline H 10 & 0.14 & 0.09 & 0.63 & 0.23 & 0.07 & 0.07 & 0.02 & 0.10 & 0.13 & 0.61 & 0.21 & 0.50 & 0.33 & 0.77 & 0.35 & 0.80 \\
\hline H 11 & 0.14 & 0.28 & 0.11 & 0.25 & 0.19 & 0.12 & 0.02 & 0.07 & 0.07 & 0.20 & 0.12 & 0.25 & 0.07 & 0.09 & 0.00 & 0.06 \\
\hline H $12 a-c$ & 0.00 & 0.08 & 0.02 & 0.17 & 0.06 & 0.01 & 0.05 & 0.03 & 0.01 & 0.01 & 0.01 & 0.08 & 0.17 & 0.05 & 0.16 & 0.07 \\
\hline H 13a & 0.05 & 0.22 & 0.35 & 0.24 & 0.18 & 0.22 & 0.01 & 0.22 & 0.11 & 0.20 & 0.19 & 0.14 & 0.25 & 0.39 & 0.22 & 0.37 \\
\hline H 13b & 0.01 & 0.01 & 0.02 & 0.04 & 0.03 & 0.01 & 0.03 & 0.01 & 0.02 & 0.02 & 0.03 & 0.05 & 0.07 & 0.05 & 0.08 & 0.03 \\
\hline H 14a & 0.08 & 0.06 & 0.08 & 0.03 & 0.19 & 0.17 & 0.14 & 0.15 & 0.05 & 0.18 & 0.12 & 0.14 & 0.01 & 0.07 & 0.03 & 0.03 \\
\hline H 14b & 0.06 & 0.25 & 0.29 & 0.27 & 0.05 & 0.01 & 0.02 & 0.01 & 0.02 & 0.02 & 0.03 & 0.00 & 0.35 & 0.31 & 0.38 & 0.29 \\
\hline H 15 & 0.06 & 0.16 & 0.22 & 0.09 & 0.12 & 0.01 & 0.13 & 0.07 & 0.07 & 0.24 & 0.00 & 0.23 & 0.04 & 0.32 & 0.05 & 0.23 \\
\hline H 17a-c & 0.07 & 0.16 & 0.11 & 0.09 & 0.02 & 0.07 & 0.04 & 0.05 & 0.12 & 0.14 & 0.12 & 0.18 & 0.19 & 0.15 & 0.20 & 0.16 \\
\hline H 18a-c & 0.19 & 0.13 & 0.23 & 0.18 & 0.18 & 0.19 & 0.21 & 0.18 & 0.08 & 0.13 & 0.12 & 0.15 & 0.20 & 0.18 & 0.24 & 0.19 \\
\hline H 19a-c & 0.09 & 0.10 & 0.05 & 0.02 & 0.00 & 0.15 & 0.03 & 0.04 & 0.06 & 0.20 & 0.00 & 0.10 & 0.16 & 0.21 & 0.06 & 0.05 \\
\hline H 20a & 0.08 & 0.09 & 0.04 & 0.05 & 0.02 & 0.03 & 0.07 & 0.08 & 0.00 & 0.02 & 0.05 & 0.06 & 0.08 & 0.00 & 0.11 & 0.06 \\
\hline $\mathrm{H} 20 \mathrm{~b}$ & 0.05 & 0.04 & 0.04 & 0.09 & 0.07 & 0.02 & 0.12 & 0.07 & 0.07 & 0.01 & 0.08 & 0.09 & 0.03 & 0.02 & 0.08 & 0.17 \\
\hline Average & 0.13 & 0.15 & 0.18 & 0.16 & 0.12 & 0.13 & 0.15 & 0.13 & 0.08 & 0.16 & 0.10 & 0.17 & 0.14 & 0.21 & 0.16 & 0.22 \\
\hline
\end{tabular}


Table S12. Unscaled and scaled chemical shifts and its errors computed for isomers 1-32 at the PCM/mPW1PW91/6-31+G**//B3LYP/6-31G* level of theory for compound 4

\begin{tabular}{|c|c|c|c|c|c|c|c|c|}
\hline \multicolumn{9}{|c|}{ Unscaled chemical shifts constants } \\
\hline Nuclei & Isomer 1 & Isomer 2 & Isomer 3 & Isomer 4 & Isomer 5 & Isomer 6 & Isomer 7 & Isomer 8 \\
\hline C 1 & 35.68 & 34.88 & 33.34 & 32.96 & 32.70 & 35.18 & 34.13 & 33.45 \\
\hline $\mathrm{C} 2$ & 30.00 & 28.84 & 28.44 & 28.02 & 29.60 & 28.31 & 30.08 & 30.20 \\
\hline C 3 & 37.92 & 30.08 & 29.92 & 26.01 & 33.23 & 33.38 & 28.04 & 27.35 \\
\hline C 4 & 77.64 & 77.65 & 77.68 & 78.82 & 76.83 & 75.90 & 78.74 & 78.47 \\
\hline C 5 & 34.67 & 34.55 & 33.82 & 30.57 & 36.04 & 39.16 & 34.94 & 34.97 \\
\hline C 6 & 86.38 & 86.39 & 86.24 & 85.97 & 87.65 & 85.89 & 87.30 & 86.08 \\
\hline$C 7$ & 35.94 & 36.68 & 36.88 & 36.39 & 36.90 & 35.35 & 37.49 & 36.86 \\
\hline C 8 & 32.43 & 32.99 & 34.99 & 35.31 & 34.20 & 33.96 & 34.09 & 32.13 \\
\hline C 9 & 39.55 & 38.26 & 39.18 & 38.44 & 40.03 & 39.27 & 37.86 & 35.76 \\
\hline C 10 & 25.24 & 30.43 & 30.01 & 32.55 & 29.90 & 28.48 & 33.75 & 33.07 \\
\hline C 11 & 43.00 & 43.47 & 39.05 & 38.35 & 41.10 & 41.25 & 37.85 & 38.72 \\
\hline C 12 & 19.24 & 19.04 & 15.99 & 15.42 & 16.16 & 16.02 & 16.73 & 15.84 \\
\hline C 13 & 30.64 & 30.22 & 29.19 & 29.02 & 29.60 & 29.17 & 29.74 & 29.09 \\
\hline C 14 & 27.99 & 27.30 & 22.38 & 22.61 & 22.59 & 21.43 & 22.77 & 22.82 \\
\hline C 15 & 77.23 & 76.66 & 76.76 & 76.74 & 77.05 & 76.55 & 76.93 & 76.80 \\
\hline C 16 & 73.97 & 73.81 & 74.00 & 73.90 & 74.36 & 74.15 & 73.85 & 73.84 \\
\hline C 17 & 27.02 & 26.03 & 24.57 & 27.22 & 26.90 & 28.81 & 27.11 & 27.38 \\
\hline C 18 & 24.55 & 26.66 & 27.27 & 24.06 & 24.35 & 24.93 & 23.95 & 23.97 \\
\hline C 19 & 20.26 & 19.76 & 19.37 & 19.61 & 19.24 & 19.09 & 21.18 & 20.53 \\
\hline C 20 & 22.81 & 22.23 & 23.07 & 23.76 & 21.97 & 23.61 & 22.53 & 23.88 \\
\hline $\mathrm{COCH}_{3}$ & 51.32 & 51.17 & 51.04 & 51.65 & 50.23 & 51.63 & 50.34 & 51.72 \\
\hline H 1 & 1.74 & 1.78 & 1.94 & 1.90 & 2.09 & 1.93 & 1.97 & 1.82 \\
\hline $\mathrm{H} 2 \mathrm{a}$ & 1.21 & 1.34 & 1.43 & 1.80 & 1.56 & 1.56 & 1.31 & 1.37 \\
\hline $\mathrm{H} 2 \mathrm{~b}$ & 1.09 & 1.04 & 1.19 & 0.76 & 1.18 & 1.08 & 1.19 & 1.14 \\
\hline H $3 a$ & 1.56 & 1.58 & 1.47 & 2.10 & 1.35 & 1.31 & 2.14 & 2.20 \\
\hline $\mathrm{H} 3 \mathrm{~b}$ & 1.22 & 1.21 & 1.21 & 0.77 & 1.21 & 1.19 & 0.92 & 0.79 \\
\hline H 5 & 1.56 & 1.56 & 1.18 & 0.94 & 1.71 & 1.44 & 1.12 & 0.73 \\
\hline H 7a & 1.53 & 1.55 & 1.48 & 1.59 & 1.63 & 1.54 & 1.53 & 1.50 \\
\hline $\mathrm{H} 7 \mathrm{~b}$ & 1.46 & 1.50 & 1.47 & 1.45 & 1.27 & 1.42 & 1.43 & 1.46 \\
\hline $\mathrm{H} 8 \mathrm{a}$ & 2.01 & 1.81 & 1.80 & 1.75 & 1.86 & 1.88 & 2.04 & 1.72 \\
\hline $\mathrm{H} 8 \mathrm{~b}$ & 1.59 & 1.72 & 1.71 & 1.60 & 1.65 & 1.69 & 1.63 & 1.66 \\
\hline H 10 & 1.05 & 1.23 & 1.43 & 1.36 & 0.87 & 1.33 & 1.03 & 1.36 \\
\hline H 11 & 1.47 & 1.54 & 1.47 & 1.42 & 1.42 & 1.44 & 1.41 & 1.44 \\
\hline H 12a-c & 0.86 & 0.91 & 1.08 & 1.30 & 0.98 & 0.97 & 1.18 & 1.27 \\
\hline H 13a & 1.59 & 1.62 & 2.07 & 1.96 & 2.08 & 2.19 & 1.92 & 2.01 \\
\hline $\mathrm{H} 13 \mathrm{~b}$ & 1.47 & 1.51 & 1.45 & 1.41 & 1.40 & 1.41 & 1.39 & 1.36 \\
\hline H 14a & 1.42 & 1.51 & 1.63 & 1.71 & 1.59 & 1.75 & 1.65 & 1.68 \\
\hline $\mathrm{H} 14 \mathrm{~b}$ & 1.41 & 1.42 & 1.22 & 1.18 & 1.14 & 1.12 & 1.15 & 1.14 \\
\hline H 15 & 3.83 & 3.68 & 3.71 & 3.48 & 3.68 & 4.10 & 3.46 & 3.44 \\
\hline H 17a-c & 1.15 & 1.06 & 1.05 & 1.09 & 1.13 & 1.22 & 1.11 & 1.12 \\
\hline H 18a-c & 1.03 & 1.10 & 1.13 & 1.09 & 1.04 & 1.00 & 1.07 & 1.08 \\
\hline H 19a-c & 0.94 & 0.93 & 0.87 & 0.78 & 1.02 & 0.97 & 1.01 & 0.94 \\
\hline H 20a-c & 1.29 & 1.27 & 1.24 & 1.22 & 1.32 & 1.22 & 1.32 & 1.30 \\
\hline $\mathrm{H} \mathrm{OCH}_{3}$ & 3.31 & 3.33 & 3.31 & 3.36 & 3.26 & 3.28 & 3.33 & 3.30 \\
\hline
\end{tabular}




\begin{tabular}{|c|c|c|c|c|c|c|c|c|}
\hline \multicolumn{9}{|c|}{ Unscaled chemical shifts constants } \\
\hline Nuclei & Isomer 9 & Isomer 10 & Isomer 11 & Isomer 12 & Isomer 13 & Isomer 14 & Isomer 15 & Isomer 16 \\
\hline C 1 & 34.92 & 36.11 & 32.11 & 32.01 & 34.49 & 34.59 & 33.69 & 33.78 \\
\hline$C 2$ & 30.05 & 30.05 & 27.20 & 27.65 & 29.18 & 28.18 & 29.81 & 29.26 \\
\hline C 3 & 38.34 & 37.85 & 18.20 & 18.65 & 32.78 & 32.66 & 20.48 & 20.26 \\
\hline C 4 & 77.90 & 77.65 & 80.38 & 80.80 & 78.65 & 77.56 & 80.39 & 80.14 \\
\hline C 5 & 33.18 & 35.33 & 32.14 & 38.43 & 34.49 & 37.77 & 37.93 & 42.07 \\
\hline C 6 & 87.03 & 86.06 & 87.85 & 86.35 & 88.35 & 85.90 & 87.69 & 85.90 \\
\hline C 7 & 36.64 & 35.59 & 39.73 & 33.07 & 37.30 & 35.71 & 38.17 & 32.86 \\
\hline C 8 & 32.60 & 32.25 & 36.25 & 35.99 & 34.69 & 33.06 & 33.31 & 33.61 \\
\hline C 9 & 41.29 & 38.71 & 36.31 & 36.17 & 40.00 & 38.51 & 35.72 & 33.01 \\
\hline C 10 & 26.13 & 24.39 & 34.47 & 34.25 & 24.46 & 24.72 & 34.84 & 34.32 \\
\hline C 11 & 43.00 & 43.15 & 42.75 & 43.53 & 42.74 & 43.36 & 43.13 & 43.34 \\
\hline C 12 & 19.32 & 19.47 & 19.32 & 19.05 & 18.81 & 20.34 & 19.28 & 18.52 \\
\hline C 13 & 30.90 & 30.65 & 31.27 & 31.35 & 30.00 & 29.85 & 31.11 & 31.31 \\
\hline C 14 & 27.95 & 28.52 & 29.03 & 29.25 & 29.28 & 27.80 & 28.98 & 28.90 \\
\hline C 15 & 77.96 & 76.94 & 77.68 & 76.49 & 76.53 & 76.48 & 77.39 & 77.12 \\
\hline C 16 & 73.46 & 74.17 & 73.43 & 73.80 & 74.51 & 73.54 & 73.47 & 73.58 \\
\hline C 17 & 26.54 & 27.20 & 27.30 & 27.57 & 26.45 & 28.64 & 27.27 & 27.04 \\
\hline C 18 & 24.60 & 24.55 & 24.09 & 24.38 & 24.10 & 25.50 & 23.96 & 23.74 \\
\hline C 19 & 20.49 & 20.19 & 19.90 & 20.48 & 19.01 & 18.74 & 21.79 & 21.65 \\
\hline C 20 & 21.64 & 23.38 & 18.81 & 22.72 & 22.30 & 23.41 & 21.89 & 22.75 \\
\hline $\mathrm{COCH}_{3}$ & 50.22 & 51.86 & 49.70 & 51.10 & 50.91 & 51.75 & 50.92 & 50.97 \\
\hline H 1 & 1.80 & 1.71 & 2.02 & 1.97 & 2.10 & 1.83 & 2.07 & 1.92 \\
\hline $\mathrm{H} 2 \mathrm{a}$ & 1.28 & 1.18 & 2.04 & 1.96 & 1.67 & 1.59 & 1.51 & 1.41 \\
\hline $\mathrm{H} 2 \mathrm{~b}$ & 1.13 & 1.05 & 0.76 & 0.75 & 1.09 & 1.08 & 1.17 & 1.16 \\
\hline H $3 a$ & 1.61 & 1.59 & 1.85 & 1.76 & 1.85 & 1.79 & 1.93 & 1.90 \\
\hline $\mathrm{H} 3 \mathrm{~b}$ & 1.21 & 1.20 & 1.07 & 0.97 & 0.93 & 0.97 & 1.02 & 1.00 \\
\hline H 5 & 1.76 & 1.48 & 1.53 & 1.03 & 1.77 & 1.48 & 1.27 & 0.88 \\
\hline H 7a & 1.58 & 1.51 & 1.45 & 1.52 & 1.69 & 1.65 & 1.50 & 1.53 \\
\hline $\mathrm{H} 7 \mathrm{~b}$ & 1.20 & 1.58 & 1.28 & 1.42 & 1.30 & 1.56 & 1.23 & 1.39 \\
\hline H 8 a & 2.11 & 1.97 & 1.98 & 1.85 & 1.87 & 1.73 & 2.10 & 1.78 \\
\hline $\mathrm{H} 8 \mathrm{~b}$ & 1.43 & 1.66 & 1.76 & 1.66 & 1.72 & 1.59 & 1.76 & 1.76 \\
\hline H 10 & 0.72 & 1.19 & 0.74 & 1.23 & 0.81 & 1.30 & 0.69 & 1.24 \\
\hline H 11 & 1.43 & 1.49 & 1.76 & 1.89 & 1.43 & 1.47 & 1.72 & 1.85 \\
\hline H 12a-c & 0.87 & 0.86 & 0.90 & 0.94 & 0.85 & 0.92 & 0.90 & 0.94 \\
\hline H 13a & 1.49 & 1.60 & 1.41 & 1.48 & 1.47 & 1.60 & 1.40 & 1.47 \\
\hline H 13b & 1.43 & 1.47 & 1.41 & 1.41 & 1.44 & 1.50 & 1.40 & 1.36 \\
\hline H 14a & 1.45 & 1.43 & 1.45 & 1.47 & 1.46 & 1.51 & 1.43 & 1.46 \\
\hline $\mathrm{H} 14 \mathrm{~b}$ & 1.42 & 1.39 & 1.40 & 1.47 & 1.37 & 1.46 & 1.40 & 1.45 \\
\hline H 15 & 3.55 & 3.96 & 3.38 & 3.39 & 3.70 & 3.99 & 3.40 & 3.30 \\
\hline H 17a-c & 1.16 & 1.14 & 1.11 & 1.10 & 1.13 & 1.19 & 1.13 & 1.11 \\
\hline H 18a-c & 1.05 & 1.01 & 1.03 & 1.06 & 1.01 & 0.97 & 1.04 & 1.05 \\
\hline H 19a-c & 0.96 & 0.93 & 0.82 & 0.82 & 1.05 & 0.95 & 1.05 & 0.95 \\
\hline H 20a-c & 1.33 & 1.28 & 1.24 & 1.23 & 1.42 & 1.28 & 1.24 & 1.28 \\
\hline $\mathrm{H} \mathrm{OCH}_{3}$ & 3.33 & 3.31 & 3.23 & 3.23 & 3.31 & 3.37 & 3.26 & 3.20 \\
\hline
\end{tabular}




\begin{tabular}{|c|c|c|c|c|c|c|c|c|}
\hline \multicolumn{9}{|c|}{ Unscaled chemical shifts constants } \\
\hline Nuclei & Isomer 17 & Isomer 18 & Isomer 19 & Isomer 20 & Isomer 21 & Isomer 22 & Isomer 23 & Isomer 24 \\
\hline C 1 & 35.47 & 35.65 & 32.05 & 31.87 & 35.37 & 34.89 & 33.18 & 33.09 \\
\hline C 2 & 28.88 & 29.31 & 27.46 & 27.66 & 27.89 & 27.57 & 29.91 & 30.44 \\
\hline C 3 & 24.62 & 24.81 & 32.01 & 31.99 & 18.99 & 18.32 & 34.70 & 33.98 \\
\hline C 4 & 78.33 & 77.26 & 79.89 & 79.02 & 78.27 & 78.14 & 78.84 & 78.89 \\
\hline C 5 & 29.56 & 36.64 & 31.84 & 38.37 & 33.04 & 34.80 & 36.34 & 42.66 \\
\hline C 6 & 87.27 & 85.31 & 87.17 & 86.68 & 87.26 & 85.90 & 87.00 & 85.92 \\
\hline C 7 & 39.47 & 35.17 & 39.61 & 33.25 & 38.52 & 37.67 & 38.68 & 33.64 \\
\hline C 8 & 32.04 & 33.03 & 36.69 & 35.57 & 35.19 & 33.01 & 33.54 & 33.61 \\
\hline C 9 & 39.13 & 38.40 & 37.57 & 34.82 & 38.92 & 37.42 & 36.81 & 33.62 \\
\hline C 10 & 35.68 & 34.68 & 25.49 & 25.71 & 36.29 & 33.91 & 26.96 & 26.05 \\
\hline C 11 & 44.28 & 44.04 & 45.66 & 44.91 & 46.28 & 45.32 & 45.15 & 45.71 \\
\hline C 12 & 18.96 & 19.40 & 20.13 & 20.17 & 19.97 & 19.83 & 20.00 & 19.07 \\
\hline C 13 & 30.60 & 30.60 & 31.82 & 31.63 & 30.37 & 30.42 & 31.51 & 31.04 \\
\hline C 14 & 29.10 & 28.54 & 28.84 & 29.69 & 29.55 & 29.11 & 29.36 & 29.22 \\
\hline C 15 & 76.65 & 76.68 & 77.34 & 77.37 & 77.11 & 76.76 & 77.00 & 77.13 \\
\hline C 16 & 73.42 & 74.42 & 74.06 & 73.80 & 73.56 & 73.82 & 73.98 & 73.82 \\
\hline C 17 & 24.40 & 24.36 & 24.15 & 24.08 & 24.53 & 24.64 & 24.07 & 24.10 \\
\hline C 18 & 27.69 & 27.80 & 27.35 & 26.95 & 27.53 & 27.72 & 27.28 & 27.30 \\
\hline C 19 & 20.71 & 19.90 & 19.39 & 20.17 & 19.46 & 19.52 & 22.71 & 22.19 \\
\hline C 20 & 19.97 & 22.76 & 20.44 & 23.22 & 22.10 & 23.91 & 22.43 & 22.85 \\
\hline $\mathrm{COCH}_{3}$ & 50.06 & 51.02 & 49.03 & 50.99 & 50.84 & 52.00 & 50.12 & 50.68 \\
\hline H 1 & 1.82 & 1.69 & 1.95 & 1.90 & 2.03 & 1.91 & 2.06 & 1.95 \\
\hline $\mathrm{H} 2 \mathrm{a}$ & 1.21 & 1.20 & 2.13 & 2.15 & 1.49 & 1.44 & 1.60 & 1.59 \\
\hline $\mathrm{H} 2 \mathrm{~b}$ & 0.92 & 0.88 & 0.83 & 0.80 & 1.10 & 1.04 & 1.34 & 1.27 \\
\hline H 3a & 1.88 & 1.85 & 1.93 & 1.74 & 1.63 & 1.59 & 1.75 & 1.82 \\
\hline $\mathrm{H} 3 \mathrm{~b}$ & 1.02 & 0.97 & 1.15 & 1.12 & 1.23 & 1.22 & 1.14 & 1.15 \\
\hline H 5 & 1.98 & 1.58 & 1.52 & 1.09 & 1.87 & 1.53 & 1.27 & 1.05 \\
\hline $\mathrm{H} 7 \mathrm{a}$ & 1.45 & 1.56 & 1.43 & 1.50 & 1.55 & 1.47 & 1.40 & 1.52 \\
\hline $\mathrm{H} 7 \mathrm{~b}$ & 1.38 & 1.50 & 1.30 & 1.49 & 1.41 & 1.43 & 1.33 & 1.45 \\
\hline $\mathrm{H} 8 \mathrm{a}$ & 2.15 & 1.94 & 1.90 & 1.75 & 1.85 & 1.85 & 1.95 & 1.79 \\
\hline $\mathrm{H} 8 \mathrm{~b}$ & 1.45 & 1.69 & 1.74 & 1.68 & 1.73 & 1.60 & 1.60 & 1.78 \\
\hline H 10 & 0.91 & 1.29 & 1.40 & 1.91 & 0.98 & 1.32 & 1.43 & 1.89 \\
\hline H 11 & 1.62 & 1.67 & 1.50 & 1.48 & 1.62 & 1.67 & 1.50 & 1.39 \\
\hline H 12a-c & 0.87 & 0.90 & 0.96 & 0.97 & 0.90 & 0.92 & 0.94 & 1.00 \\
\hline H 13a & 1.49 & 1.51 & 1.62 & 1.73 & 1.51 & 1.52 & 1.67 & 1.74 \\
\hline $\mathrm{H} 13 \mathrm{~b}$ & 1.40 & 1.39 & 1.49 & 1.46 & 1.35 & 1.41 & 1.49 & 1.46 \\
\hline H 14a & 1.51 & 1.50 & 1.54 & 1.52 & 1.52 & 1.52 & 1.51 & 1.52 \\
\hline $\mathrm{H} 14 \mathrm{~b}$ & 1.44 & 1.39 & 1.44 & 1.44 & 1.40 & 1.43 & 1.48 & 1.39 \\
\hline H 15 & 3.34 & 3.32 & 3.68 & 3.78 & 3.43 & 3.39 & 3.72 & 3.75 \\
\hline H 17a-c & 1.06 & 1.03 & 1.08 & 1.06 & 1.06 & 1.03 & 1.10 & 1.06 \\
\hline H 18a-c & 1.11 & 1.09 & 1.13 & 1.14 & 1.12 & 1.10 & 1.17 & 1.12 \\
\hline H 19a-c & 0.95 & 0.95 & 0.81 & 0.83 & 1.01 & 0.97 & 0.98 & 0.96 \\
\hline H 20a-c & 1.25 & 1.24 & 1.26 & 1.22 & 1.25 & 1.25 & 1.22 & 1.20 \\
\hline $\mathrm{H} \mathrm{OCH}_{3}$ & 3.38 & 3.24 & 3.23 & 3.23 & 3.31 & 3.42 & 3.29 & 3.20 \\
\hline
\end{tabular}




\begin{tabular}{|c|c|c|c|c|c|c|c|c|}
\hline \multicolumn{9}{|c|}{ Unscaled chemical shifts constants } \\
\hline Nuclei & Isomer 25 & Isomer 26 & Isomer 27 & Isomer 28 & Isomer 29 & Isomer 30 & Isomer 31 & Isomer 32 \\
\hline C 1 & 35.63 & 34.12 & 32.70 & 32.52 & 34.98 & 34.15 & 34.46 & 35.51 \\
\hline $\mathrm{C} 2$ & 28.98 & 30.18 & 28.40 & 28.52 & 30.05 & 30.50 & 30.50 & 30.91 \\
\hline C 3 & 32.13 & 29.38 & 31.75 & 31.34 & 26.96 & 27.73 & 33.70 & 33.52 \\
\hline C 4 & 76.04 & 76.63 & 77.76 & 77.57 & 77.09 & 77.86 & 77.66 & 77.49 \\
\hline C 5 & 29.62 & 33.61 & 32.11 & 33.12 & 33.83 & 34.70 & 36.28 & 37.72 \\
\hline C 6 & 87.44 & 86.61 & 87.10 & 85.30 & 87.22 & 85.88 & 87.08 & 85.18 \\
\hline C 7 & 38.95 & 37.10 & 38.46 & 37.91 & 38.31 & 37.96 & 38.25 & 37.00 \\
\hline C 8 & 33.98 & 34.63 & 36.61 & 35.05 & 34.68 & 32.80 & 34.30 & 32.03 \\
\hline C 9 & 37.74 & 38.09 & 40.31 & 39.68 & 38.95 & 38.72 & 38.99 & 38.78 \\
\hline C 10 & 32.83 & 31.66 & 28.75 & 26.73 & 31.80 & 30.54 & 28.90 & 26.86 \\
\hline C 11 & 40.32 & 37.01 & 37.58 & 38.39 & 37.89 & 37.64 & 37.88 & 38.75 \\
\hline C 12 & 16.75 & 16.64 & 16.21 & 16.24 & 17.16 & 16.97 & 16.36 & 16.36 \\
\hline C 13 & 29.58 & 29.62 & 29.75 & 28.73 & 29.15 & 29.86 & 29.67 & 28.87 \\
\hline C 14 & 22.72 & 22.84 & 22.94 & 22.57 & 23.08 & 23.13 & 21.88 & 22.38 \\
\hline C 15 & 76.93 & 77.32 & 76.84 & 76.68 & 77.05 & 77.23 & 77.39 & 77.59 \\
\hline C 16 & 73.71 & 74.11 & 73.95 & 73.83 & 74.04 & 74.09 & 74.49 & 74.06 \\
\hline C 17 & 24.20 & 24.00 & 24.15 & 24.20 & 24.22 & 24.27 & 24.15 & 23.98 \\
\hline C 18 & 27.81 & 27.40 & 27.33 & 27.33 & 27.30 & 27.90 & 27.42 & 27.27 \\
\hline C 19 & 20.52 & 18.73 & 19.23 & 19.02 & 20.35 & 20.14 & 21.56 & 20.81 \\
\hline C 20 & 20.13 & 23.26 & 20.70 & 23.74 & 21.70 & 23.54 & 21.59 & 24.04 \\
\hline $\mathrm{COCH}_{3}$ & 49.83 & 51.45 & 49.27 & 51.44 & 50.64 & 52.06 & 50.39 & 51.77 \\
\hline H 1 & 1.78 & 1.75 & 2.01 & 1.92 & 2.01 & 1.92 & 2.01 & 1.77 \\
\hline $\mathrm{H} 2 \mathrm{a}$ & 1.24 & 1.32 & 1.87 & 1.85 & 1.47 & 1.41 & 1.41 & 1.34 \\
\hline $\mathrm{H} 2 \mathrm{~b}$ & 0.92 & 0.93 & 0.87 & 0.81 & 1.02 & 1.00 & 1.26 & 1.16 \\
\hline H $3 a$ & 2.45 & 1.69 & 1.42 & 1.39 & 1.71 & 1.60 & 1.48 & 1.53 \\
\hline$H 3 b$ & 0.82 & 1.35 & 1.15 & 1.10 & 1.53 & 1.47 & 1.19 & 1.05 \\
\hline H 5 & 1.96 & 1.33 & 1.20 & 0.92 & 1.71 & 1.25 & 1.07 & 0.79 \\
\hline $\mathrm{H} 7 \mathrm{a}$ & 1.44 & 1.52 & 1.47 & 1.50 & 1.51 & 1.48 & 1.51 & 1.52 \\
\hline $\mathrm{H} 7 \mathrm{~b}$ & 1.37 & 1.49 & 1.32 & 1.44 & 1.30 & 1.48 & 1.36 & 1.45 \\
\hline H 8a & 2.12 & 1.82 & 1.96 & 1.79 & 1.94 & 1.81 & 2.04 & 1.78 \\
\hline $\mathrm{H} 8 \mathrm{~b}$ & 1.46 & 1.76 & 1.68 & 1.73 & 1.68 & 1.64 & 1.63 & 1.73 \\
\hline H 10 & 1.06 & 1.55 & 1.72 & 1.95 & 1.21 & 1.58 & 1.50 & 1.87 \\
\hline H 11 & 1.45 & 1.68 & 1.59 & 1.60 & 1.72 & 1.74 & 1.66 & 1.66 \\
\hline H 12a-c & 1.22 & 1.22 & 0.95 & 0.93 & 1.18 & 1.23 & 0.94 & 0.94 \\
\hline $\mathrm{H} 13 a$ & 2.02 & 1.97 & 2.15 & 2.38 & 1.95 & 1.93 & 2.08 & 2.31 \\
\hline $\mathrm{H} 13 \mathrm{~b}$ & 1.41 & 1.39 & 1.44 & 1.38 & 1.44 & 1.43 & 1.40 & 1.36 \\
\hline H 14a & 1.78 & 1.64 & 1.64 & 1.62 & 1.70 & 1.72 & 1.63 & 1.63 \\
\hline $\mathrm{H} 14 \mathrm{~b}$ & 1.16 & 1.13 & 1.22 & 1.23 & 1.17 & 1.17 & 1.19 & 1.26 \\
\hline H 15 & 3.41 & 3.43 & 3.68 & 3.77 & 3.46 & 3.49 & 3.69 & 3.70 \\
\hline H 17a-c & 1.06 & 1.09 & 1.06 & 1.05 & 1.05 & 1.04 & 1.09 & 1.09 \\
\hline H 18a-c & 1.12 & 1.06 & 1.11 & 1.11 & 1.11 & 1.09 & 1.16 & 1.12 \\
\hline H 19a-c & 0.94 & 0.87 & 0.85 & 0.81 & 0.98 & 0.92 & 1.01 & 0.98 \\
\hline H 20a-c & 1.27 & 1.27 & 1.23 & 1.24 & 1.26 & 1.20 & 1.22 & 1.23 \\
\hline $\mathrm{HOCH}_{3}$ & 3.38 & 3.30 & 3.28 & 3.34 & 3.30 & 3.39 & 3.32 & 3.33 \\
\hline
\end{tabular}




\begin{tabular}{|c|c|c|c|c|c|c|c|c|}
\hline \multicolumn{9}{|c|}{ Scaled chemical shifts constants } \\
\hline Nuclei & Isomer 1 & Isomer 2 & Isomer 3 & Isomer 4 & Isomer $\mathbf{5}$ & Isomer 6 & Isomer 7 & Isomer 8 \\
\hline C 1 & 33.07 & 32.63 & 31.84 & 31.82 & 30.83 & 33.18 & 32.34 & 31.98 \\
\hline $\mathrm{C} 2$ & 27.22 & 26.49 & 26.94 & 26.94 & 27.72 & 26.15 & 28.29 & 28.73 \\
\hline $\mathrm{C} 3$ & 35.38 & 27.75 & 28.42 & 24.96 & 31.36 & 31.33 & 26.25 & 25.88 \\
\hline $\mathrm{C} 4$ & 76.28 & 76.11 & 76.20 & 77.11 & 75.09 & 74.84 & 76.97 & 76.95 \\
\hline $\mathrm{C} 5$ & 32.04 & 32.29 & 32.32 & 29.46 & 34.18 & 37.25 & 33.15 & 33.49 \\
\hline C 6 & 85.27 & 85.00 & 84.78 & 84.17 & 85.94 & 85.06 & 85.53 & 84.55 \\
\hline$C 7$ & 33.34 & 34.46 & 35.38 & 35.21 & 35.05 & 33.35 & 35.70 & 35.38 \\
\hline C 8 & 29.73 & 30.71 & 33.50 & 34.15 & 32.33 & 31.93 & 32.30 & 30.66 \\
\hline C 9 & 37.06 & 36.07 & 37.68 & 37.24 & 38.18 & 37.36 & 36.07 & 34.28 \\
\hline C 10 & 22.32 & 28.11 & 28.51 & 31.42 & 28.02 & 26.32 & 31.96 & 31.60 \\
\hline C 11 & 40.61 & 41.37 & 37.56 & 37.15 & 39.25 & 39.38 & 36.05 & 37.23 \\
\hline C 12 & 16.15 & 16.53 & 14.48 & 14.50 & 14.24 & 13.57 & 14.93 & 14.38 \\
\hline C 13 & 27.88 & 27.90 & 27.68 & 27.93 & 27.72 & 27.02 & 27.94 & 27.61 \\
\hline C 14 & 25.15 & 24.93 & 20.87 & 21.60 & 20.69 & 19.10 & 20.98 & 21.36 \\
\hline C 15 & 75.85 & 75.11 & 75.29 & 75.06 & 75.31 & 75.51 & 75.16 & 75.29 \\
\hline C 16 & 72.50 & 72.21 & 72.52 & 72.26 & 72.61 & 73.05 & 72.07 & 72.33 \\
\hline C 17 & 24.16 & 23.64 & 23.07 & 26.15 & 25.01 & 26.65 & 25.31 & 25.91 \\
\hline C 18 & 21.61 & 24.28 & 25.77 & 23.03 & 22.46 & 22.69 & 22.15 & 22.50 \\
\hline C 19 & 17.20 & 17.26 & 17.86 & 18.64 & 17.33 & 16.71 & 19.38 & 19.07 \\
\hline C 20 & 19.82 & 19.77 & 21.57 & 22.73 & 20.07 & 21.33 & 20.73 & 22.42 \\
\hline $\mathrm{COCH}_{3}$ & 49.17 & 49.19 & 49.56 & 50.28 & 48.41 & 50.01 & 48.55 & 50.23 \\
\hline H 1 & 1.69 & 1.72 & 1.87 & 1.83 & 2.02 & 1.81 & 1.90 & 1.78 \\
\hline $\mathrm{H} 2 \mathrm{a}$ & 1.19 & 1.28 & 1.36 & 1.73 & 1.49 & 1.46 & 1.25 & 1.32 \\
\hline $\mathrm{H} 2 \mathrm{~b}$ & 1.07 & 0.99 & 1.12 & 0.74 & 1.11 & 1.02 & 1.13 & 1.10 \\
\hline H $3 a$ & 1.52 & 1.52 & 1.39 & 2.02 & 1.28 & 1.24 & 2.07 & 2.16 \\
\hline $\mathrm{H} 3 \mathrm{~b}$ & 1.20 & 1.16 & 1.14 & 0.76 & 1.14 & 1.13 & 0.87 & 0.74 \\
\hline H 5 & 1.52 & 1.50 & 1.10 & 0.91 & 1.64 & 1.35 & 1.07 & 0.68 \\
\hline $\mathrm{H} 7 \mathrm{a}$ & 1.49 & 1.49 & 1.40 & 1.54 & 1.56 & 1.45 & 1.47 & 1.46 \\
\hline $\mathrm{H} 7 \mathrm{~b}$ & 1.42 & 1.45 & 1.39 & 1.41 & 1.20 & 1.34 & 1.37 & 1.42 \\
\hline $\mathrm{H} 8 \mathrm{a}$ & 1.95 & 1.74 & 1.72 & 1.69 & 1.79 & 1.77 & 1.97 & 1.68 \\
\hline $\mathrm{H} 8 \mathrm{~b}$ & 1.55 & 1.65 & 1.63 & 1.55 & 1.58 & 1.59 & 1.56 & 1.62 \\
\hline H 10 & 1.04 & 1.18 & 1.36 & 1.32 & 0.80 & 1.26 & 0.97 & 1.32 \\
\hline H 11 & 1.43 & 1.48 & 1.40 & 1.38 & 1.35 & 1.36 & 1.35 & 1.39 \\
\hline H 12a-c & 0.86 & 0.87 & 1.01 & 1.26 & 0.91 & 0.92 & 1.12 & 1.23 \\
\hline H 13a & 1.54 & 1.56 & 1.99 & 1.90 & 2.01 & 2.05 & 1.85 & 1.97 \\
\hline $\mathrm{H} 13 \mathrm{~b}$ & 1.43 & 1.45 & 1.38 & 1.37 & 1.33 & 1.33 & 1.33 & 1.31 \\
\hline $\mathrm{H} 14 \mathrm{a}$ & 1.38 & 1.45 & 1.56 & 1.65 & 1.52 & 1.64 & 1.58 & 1.63 \\
\hline $\mathrm{H} 14 \mathrm{~b}$ & 1.38 & 1.36 & 1.14 & 1.15 & 1.07 & 1.06 & 1.09 & 1.10 \\
\hline H 15 & 3.66 & 3.57 & 3.63 & 3.34 & 3.61 & 3.81 & 3.37 & 3.40 \\
\hline H 17a-c & 1.13 & 1.01 & 0.98 & 1.07 & 1.06 & 1.15 & 1.05 & 1.07 \\
\hline H 18a-c & 1.01 & 1.05 & 1.06 & 1.06 & 0.97 & 0.95 & 1.01 & 1.04 \\
\hline H 19a-c & 0.93 & 0.88 & 0.80 & 0.76 & 0.95 & 0.93 & 0.95 & 0.89 \\
\hline H 20a-c & 1.27 & 1.22 & 1.17 & 1.18 & 1.25 & 1.16 & 1.26 & 1.26 \\
\hline $\mathrm{HOCH}_{3}$ & 3.17 & 3.24 & 3.23 & 3.22 & 3.19 & 3.06 & 3.24 & 3.27 \\
\hline
\end{tabular}


Scaled chemical shifts constants

\begin{tabular}{|c|c|c|c|c|c|c|c|c|}
\hline Nuclei & Isomer 9 & Isomer 10 & Isomer 11 & Isomer 12 & Isomer 13 & Isomer 14 & Isomer 15 & Isomer 16 \\
\hline C 1 & 32.29 & 33.47 & 30.61 & 30.09 & 32.24 & 32.22 & 31.39 & 31.74 \\
\hline$C 2$ & 27.31 & 27.21 & 25.83 & 25.76 & 26.92 & 25.61 & 27.53 & 27.20 \\
\hline C 3 & 35.79 & 35.27 & 17.07 & 16.81 & 30.53 & 30.22 & 18.26 & 18.17 \\
\hline $\mathrm{C} 4$ & 76.30 & 76.38 & 77.56 & 78.60 & 76.43 & 76.46 & 77.76 & 78.28 \\
\hline C 5 & 30.51 & 32.66 & 30.63 & 36.47 & 32.24 & 35.49 & 35.59 & 40.06 \\
\hline C 6 & 85.65 & 85.07 & 84.83 & 84.11 & 86.14 & 85.05 & 85.01 & 84.07 \\
\hline$C 7$ & 34.05 & 32.93 & 38.02 & 31.15 & 35.05 & 33.36 & 35.83 & 30.82 \\
\hline C 8 & 29.91 & 29.49 & 34.63 & 34.05 & 32.44 & 30.64 & 31.00 & 31.56 \\
\hline C 9 & 38.81 & 36.15 & 34.69 & 34.23 & 37.75 & 36.25 & 33.40 & 30.97 \\
\hline C 10 & 23.29 & 21.36 & 32.89 & 32.32 & 22.20 & 22.05 & 32.53 & 32.28 \\
\hline C 11 & 40.56 & 40.74 & 40.95 & 41.55 & 40.49 & 41.25 & 40.76 & 41.34 \\
\hline C 12 & 16.32 & 16.28 & 18.16 & 17.20 & 16.55 & 17.54 & 17.07 & 16.42 \\
\hline C 13 & 28.17 & 27.83 & 29.78 & 29.44 & 27.74 & 27.34 & 28.82 & 29.26 \\
\hline C 14 & 25.15 & 25.63 & 27.61 & 27.35 & 27.02 & 25.23 & 26.70 & 26.84 \\
\hline C 15 & 76.36 & 75.65 & 74.93 & 74.32 & 74.31 & 75.35 & 74.79 & 75.26 \\
\hline C 16 & 71.75 & 72.79 & 70.80 & 71.64 & 72.28 & 72.32 & 70.89 & 71.70 \\
\hline C 17 & 23.71 & 24.27 & 25.92 & 25.68 & 24.19 & 26.08 & 25.01 & 24.97 \\
\hline C 18 & 21.72 & 21.53 & 22.80 & 22.51 & 21.83 & 22.85 & 21.72 & 21.66 \\
\hline C 19 & 17.51 & 17.02 & 18.72 & 18.63 & 16.75 & 15.89 & 19.57 & 19.56 \\
\hline C 20 & 18.69 & 20.32 & 17.66 & 20.85 & 20.03 & 20.70 & 19.67 & 20.66 \\
\hline $\mathrm{COCH}_{3}$ & 47.95 & 49.74 & 47.71 & 49.06 & 48.67 & 49.89 & 48.50 & 49.00 \\
\hline $\mathrm{H} 1$ & 1.77 & 1.64 & 1.98 & 1.93 & 2.01 & 1.73 & 2.03 & 1.92 \\
\hline $\mathrm{H} 2 \mathrm{a}$ & 1.26 & 1.16 & 1.99 & 1.92 & 1.59 & 1.51 & 1.48 & 1.38 \\
\hline $\mathrm{H} 2 \mathrm{~b}$ & 1.12 & 1.04 & 0.75 & 0.73 & 1.05 & 1.04 & 1.14 & 1.12 \\
\hline H 3a & 1.58 & 1.54 & 1.81 & 1.72 & 1.76 & 1.69 & 1.89 & 1.89 \\
\hline $\mathrm{H} 3 \mathrm{~b}$ & 1.20 & 1.18 & 1.05 & 0.94 & 0.90 & 0.94 & 0.99 & 0.95 \\
\hline H 5 & 1.74 & 1.44 & 1.49 & 1.00 & 1.69 & 1.40 & 1.24 & 0.82 \\
\hline $\mathrm{H} 7 \mathrm{a}$ & 1.55 & 1.47 & 1.42 & 1.49 & 1.61 & 1.56 & 1.46 & 1.51 \\
\hline $\mathrm{H} 7 \mathrm{~b}$ & 1.19 & 1.53 & 1.25 & 1.39 & 1.24 & 1.48 & 1.20 & 1.36 \\
\hline H 8a & 2.08 & 1.89 & 1.94 & 1.82 & 1.78 & 1.63 & 2.07 & 1.77 \\
\hline $\mathrm{H} 8 \mathrm{~b}$ & 1.41 & 1.60 & 1.72 & 1.63 & 1.64 & 1.50 & 1.72 & 1.75 \\
\hline H 10 & 0.72 & 1.17 & 0.73 & 1.20 & 0.78 & 1.24 & 0.66 & 1.20 \\
\hline H 11 & 1.41 & 1.45 & 1.73 & 1.86 & 1.37 & 1.40 & 1.68 & 1.85 \\
\hline H 12a-c & 0.86 & 0.86 & 0.89 & 0.91 & 0.82 & 0.89 & 0.87 & 0.88 \\
\hline H 13a & 1.47 & 1.55 & 1.38 & 1.45 & 1.41 & 1.51 & 1.37 & 1.44 \\
\hline $\mathrm{H} 13 \mathrm{~b}$ & 1.41 & 1.42 & 1.38 & 1.38 & 1.38 & 1.43 & 1.36 & 1.32 \\
\hline H 14a & 1.43 & 1.39 & 1.41 & 1.44 & 1.40 & 1.43 & 1.40 & 1.44 \\
\hline $\mathrm{H} 14 \mathrm{~b}$ & 1.40 & 1.36 & 1.37 & 1.43 & 1.31 & 1.38 & 1.37 & 1.42 \\
\hline H 15 & 3.49 & 3.72 & 3.31 & 3.34 & 3.53 & 3.70 & 3.35 & 3.37 \\
\hline H 17a-c & 1.15 & 1.13 & 1.08 & 1.07 & 1.08 & 1.15 & 1.10 & 1.07 \\
\hline H 18a-c & 1.04 & 1.00 & 1.01 & 1.03 & 0.97 & 0.94 & 1.01 & 1.00 \\
\hline H 19a-c & 0.95 & 0.93 & 0.81 & 0.79 & 1.01 & 0.92 & 1.02 & 0.90 \\
\hline H 20a-c & 1.32 & 1.25 & 1.21 & 1.20 & 1.36 & 1.22 & 1.21 & 1.24 \\
\hline $\mathrm{HOCH}_{3}$ & 3.28 & 3.12 & 3.16 & 3.18 & 3.15 & 3.13 & 3.22 & 3.27 \\
\hline
\end{tabular}


Scaled chemical shifts constants

\begin{tabular}{|c|c|c|c|c|c|c|c|c|}
\hline Nuclei & Isomer 17 & Isomer 18 & Isomer 19 & Isomer 20 & Isomer 21 & Isomer 22 & Isomer 23 & Isomer 24 \\
\hline C 1 & 33.35 & 33.26 & 29.86 & 29.56 & 33.05 & 32.85 & 30.22 & 30.31 \\
\hline$C 2$ & 26.75 & 26.78 & 25.27 & 25.29 & 25.59 & 25.53 & 26.85 & 27.57 \\
\hline C 3 & 22.49 & 22.18 & 29.83 & 29.68 & 16.73 & 16.28 & 31.78 & 31.23 \\
\hline $\mathrm{C} 4$ & 76.23 & 75.78 & 77.65 & 77.36 & 75.76 & 76.09 & 77.14 & 77.60 \\
\hline$C 5$ & 27.44 & 34.27 & 29.65 & 36.15 & 30.73 & 32.76 & 33.46 & 40.19 \\
\hline C 6 & 85.18 & 84.02 & 84.91 & 85.12 & 84.72 & 83.85 & 85.52 & 84.85 \\
\hline$C 7$ & 37.35 & 32.77 & 37.41 & 30.96 & 36.18 & 35.62 & 35.87 & 30.87 \\
\hline C 8 & 29.92 & 30.58 & 34.49 & 33.31 & 32.87 & 30.97 & 30.58 & 30.84 \\
\hline C 9 & 37.00 & 36.07 & 35.37 & 32.55 & 36.58 & 35.38 & 33.94 & 30.86 \\
\hline C 10 & 33.56 & 32.27 & 23.31 & 23.31 & 33.97 & 31.87 & 23.82 & 23.03 \\
\hline C 11 & 42.17 & 41.84 & 43.45 & 42.77 & 43.91 & 43.28 & 42.51 & 43.33 \\
\hline C 12 & 16.83 & 16.65 & 17.96 & 17.69 & 17.72 & 17.79 & 16.67 & 15.83 \\
\hline C 13 & 28.48 & 28.09 & 29.63 & 29.31 & 28.07 & 28.38 & 28.50 & 28.19 \\
\hline C 14 & 26.98 & 25.99 & 26.65 & 27.35 & 27.25 & 27.07 & 26.29 & 26.32 \\
\hline C 15 & 74.55 & 75.19 & 75.10 & 75.68 & 74.60 & 74.72 & 75.25 & 75.78 \\
\hline C 16 & 71.32 & 72.89 & 71.82 & 72.07 & 71.07 & 71.77 & 72.15 & 72.36 \\
\hline C 17 & 22.28 & 21.72 & 21.97 & 21.66 & 22.26 & 22.60 & 20.85 & 21.03 \\
\hline C 18 & 25.56 & 25.24 & 25.17 & 24.57 & 25.24 & 25.68 & 24.15 & 24.33 \\
\hline C 19 & 18.57 & 17.16 & 17.21 & 17.69 & 17.21 & 17.48 & 19.46 & 19.05 \\
\hline C 20 & 17.84 & 20.09 & 18.27 & 20.78 & 19.83 & 21.87 & 19.16 & 19.73 \\
\hline $\mathrm{COCH}_{3}$ & 47.94 & 48.97 & 46.82 & 48.94 & 48.45 & 49.96 & 47.63 & 48.47 \\
\hline H 1 & 1.78 & 1.67 & 1.84 & 1.80 & 1.98 & 1.86 & 1.97 & 1.87 \\
\hline $\mathrm{H} 2 \mathrm{a}$ & 1.19 & 1.17 & 2.03 & 2.04 & 1.42 & 1.39 & 1.51 & 1.50 \\
\hline $\mathrm{H} 2 \mathrm{~b}$ & 0.91 & 0.84 & 0.75 & 0.72 & 1.03 & 0.98 & 1.25 & 1.17 \\
\hline H $3 a$ & 1.83 & 1.83 & 1.83 & 1.64 & 1.57 & 1.54 & 1.66 & 1.73 \\
\hline $\mathrm{H} 3 \mathrm{~b}$ & 1.00 & 0.93 & 1.07 & 1.03 & 1.15 & 1.16 & 1.05 & 1.04 \\
\hline H 5 & 1.94 & 1.56 & 1.43 & 1.01 & 1.81 & 1.47 & 1.18 & 0.93 \\
\hline H 7a & 1.42 & 1.54 & 1.34 & 1.41 & 1.48 & 1.41 & 1.31 & 1.42 \\
\hline $\mathrm{H} 7 \mathrm{~b}$ & 1.35 & 1.48 & 1.21 & 1.40 & 1.34 & 1.38 & 1.24 & 1.35 \\
\hline H 8 a & 2.10 & 1.93 & 1.80 & 1.65 & 1.79 & 1.80 & 1.86 & 1.70 \\
\hline $\mathrm{H} 8 \mathrm{~b}$ & 1.42 & 1.67 & 1.65 & 1.58 & 1.67 & 1.55 & 1.51 & 1.69 \\
\hline H 10 & 0.89 & 1.26 & 1.31 & 1.81 & 0.90 & 1.27 & 1.33 & 1.81 \\
\hline H 11 & 1.58 & 1.65 & 1.40 & 1.38 & 1.55 & 1.62 & 1.41 & 1.29 \\
\hline H 12a-c & 0.86 & 0.86 & 0.88 & 0.89 & 0.81 & 0.86 & 0.85 & 0.88 \\
\hline H 13a & 1.46 & 1.48 & 1.52 & 1.64 & 1.44 & 1.47 & 1.58 & 1.64 \\
\hline $\mathrm{H} 13 \mathrm{~b}$ & 1.38 & 1.37 & 1.40 & 1.37 & 1.28 & 1.36 & 1.40 & 1.36 \\
\hline H 14a & 1.48 & 1.47 & 1.44 & 1.43 & 1.46 & 1.47 & 1.42 & 1.42 \\
\hline H 14b & 1.41 & 1.36 & 1.35 & 1.35 & 1.33 & 1.38 & 1.39 & 1.29 \\
\hline H 15 & 3.26 & 3.33 & 3.54 & 3.65 & 3.41 & 3.37 & 3.63 & 3.72 \\
\hline H 17a-c & 1.04 & 1.00 & 0.99 & 0.98 & 0.98 & 0.97 & 1.00 & 0.94 \\
\hline H 18a-c & 1.09 & 1.06 & 1.05 & 1.05 & 1.04 & 1.04 & 1.08 & 1.00 \\
\hline H 19a-c & 0.94 & 0.91 & 0.74 & 0.75 & 0.93 & 0.91 & 0.89 & 0.85 \\
\hline H 20a-c & 1.23 & 1.21 & 1.17 & 1.14 & 1.18 & 1.19 & 1.12 & 1.09 \\
\hline $\mathrm{H} \mathrm{OCH}_{3}$ & 3.30 & 3.24 & 3.10 & 3.11 & 3.29 & 3.40 & 3.21 & 3.15 \\
\hline
\end{tabular}




\begin{tabular}{|c|c|c|c|c|c|c|c|c|}
\hline \multicolumn{9}{|c|}{ Scaled chemical shifts constants } \\
\hline Nuclei & Isomer 25 & Isomer 26 & Isomer 27 & Isomer 28 & Isomer 29 & Isomer 30 & Isomer 31 & Isomer 32 \\
\hline C 1 & 33.98 & 32.55 & 31.27 & 31.14 & 33.32 & 32.42 & 32.46 & 33.57 \\
\hline $\mathrm{C} 2$ & 27.28 & 28.59 & 26.98 & 27.11 & 28.40 & 28.75 & 28.46 & 28.89 \\
\hline C 3 & 30.46 & 27.79 & 30.33 & 29.96 & 25.30 & 25.97 & 31.69 & 31.55 \\
\hline C 4 & 74.66 & 75.21 & 76.27 & 76.46 & 75.45 & 76.31 & 76.02 & 76.28 \\
\hline C 5 & 27.93 & 32.04 & 30.69 & 31.74 & 32.18 & 32.97 & 34.29 & 35.82 \\
\hline C 6 & 86.13 & 85.23 & 85.60 & 84.24 & 85.59 & 84.36 & 85.51 & 84.11 \\
\hline C 7 & 37.32 & 35.53 & 37.03 & 36.56 & 36.66 & 36.25 & 36.27 & 35.08 \\
\hline C 8 & 32.33 & 33.06 & 35.18 & 33.69 & 33.03 & 31.06 & 32.30 & 30.03 \\
\hline C 9 & 36.11 & 36.53 & 38.87 & 38.34 & 37.30 & 37.01 & 37.02 & 36.89 \\
\hline C 10 & 31.16 & 30.08 & 27.33 & 25.32 & 30.15 & 28.80 & 26.85 & 24.76 \\
\hline C 11 & 38.70 & 35.45 & 36.15 & 37.04 & 36.24 & 35.92 & 35.91 & 36.87 \\
\hline C 12 & 14.98 & 15.01 & 14.81 & 14.76 & 15.50 & 15.17 & 14.21 & 14.08 \\
\hline C 13 & 27.90 & 28.03 & 28.33 & 27.33 & 27.50 & 28.11 & 27.63 & 26.82 \\
\hline C 14 & 20.98 & 21.23 & 21.53 & 21.13 & 21.42 & 21.35 & 19.77 & 20.21 \\
\hline C 15 & 75.55 & 75.91 & 75.35 & 75.57 & 75.41 & 75.67 & 75.75 & 76.39 \\
\hline C 16 & 72.31 & 72.68 & 72.47 & 72.70 & 72.40 & 72.53 & 72.82 & 72.80 \\
\hline C 17 & 22.48 & 22.39 & 22.73 & 22.77 & 22.57 & 22.50 & 22.06 & 21.84 \\
\hline C 18 & 26.11 & 25.80 & 25.91 & 25.92 & 25.65 & 26.14 & 25.36 & 25.19 \\
\hline C 19 & 18.77 & 17.10 & 17.83 & 17.55 & 18.69 & 18.35 & 19.45 & 18.61 \\
\hline C 20 & 18.38 & 21.65 & 19.29 & 22.31 & 20.05 & 21.76 & 19.47 & 21.90 \\
\hline $\mathrm{COCH}_{3}$ & 48.27 & 49.94 & 47.82 & 50.17 & 49.00 & 50.40 & 48.52 & 50.12 \\
\hline H 1 & 1.68 & 1.68 & 1.91 & 1.81 & 1.92 & 1.83 & 1.92 & 1.69 \\
\hline $\mathrm{H} 2 \mathrm{a}$ & 1.17 & 1.23 & 1.77 & 1.75 & 1.35 & 1.31 & 1.33 & 1.26 \\
\hline $\mathrm{H} 2 \mathrm{~b}$ & 0.86 & 0.82 & 0.80 & 0.76 & 0.88 & 0.89 & 1.18 & 1.09 \\
\hline H $3 a$ & 2.32 & 1.62 & 1.33 & 1.32 & 1.60 & 1.51 & 1.40 & 1.46 \\
\hline$H 3 b$ & 0.77 & 1.27 & 1.08 & 1.03 & 1.41 & 1.37 & 1.11 & 0.98 \\
\hline H 5 & 1.86 & 1.24 & 1.12 & 0.87 & 1.60 & 1.14 & 0.99 & 0.73 \\
\hline $\mathrm{H} 7 \mathrm{a}$ & 1.36 & 1.44 & 1.39 & 1.41 & 1.39 & 1.38 & 1.42 & 1.44 \\
\hline $\mathrm{H} 7 \mathrm{~b}$ & 1.30 & 1.41 & 1.24 & 1.36 & 1.18 & 1.38 & 1.28 & 1.38 \\
\hline H 8a & 2.01 & 1.76 & 1.86 & 1.69 & 1.84 & 1.72 & 1.95 & 1.70 \\
\hline $\mathrm{H} 8 \mathrm{~b}$ & 1.38 & 1.70 & 1.59 & 1.64 & 1.57 & 1.55 & 1.54 & 1.65 \\
\hline H 10 & 1.00 & 1.48 & 1.63 & 1.85 & 1.08 & 1.48 & 1.41 & 1.79 \\
\hline H 11 & 1.37 & 1.60 & 1.50 & 1.51 & 1.61 & 1.65 & 1.57 & 1.59 \\
\hline H 12a-c & 1.15 & 1.13 & 0.88 & 0.88 & 1.04 & 1.12 & 0.86 & 0.87 \\
\hline $\mathrm{H} 13 a$ & 1.91 & 1.91 & 2.05 & 2.26 & 1.85 & 1.85 & 1.99 & 2.22 \\
\hline $\mathrm{H} 13 \mathrm{~b}$ & 1.33 & 1.31 & 1.35 & 1.30 & 1.32 & 1.33 & 1.32 & 1.29 \\
\hline H 14a & 1.69 & 1.57 & 1.55 & 1.53 & 1.59 & 1.63 & 1.54 & 1.56 \\
\hline $\mathrm{H} 14 \mathrm{~b}$ & 1.09 & 1.04 & 1.15 & 1.16 & 1.04 & 1.06 & 1.10 & 1.19 \\
\hline H 15 & 3.24 & 3.43 & 3.54 & 3.57 & 3.44 & 3.45 & 3.58 & 3.59 \\
\hline H 17a-c & 0.99 & 0.99 & 0.98 & 0.99 & 0.91 & 0.93 & 1.01 & 1.02 \\
\hline H 18a-c & 1.06 & 0.96 & 1.03 & 1.05 & 0.98 & 0.98 & 1.08 & 1.05 \\
\hline H 19a-c & 0.89 & 0.77 & 0.78 & 0.76 & 0.84 & 0.81 & 0.93 & 0.91 \\
\hline H 20a-c & 1.20 & 1.18 & 1.15 & 1.17 & 1.13 & 1.10 & 1.14 & 1.16 \\
\hline $\mathrm{H} \mathrm{OCH}_{3}$ & 3.21 & 3.30 & 3.15 & 3.16 & 3.26 & 3.35 & 3.21 & 3.23 \\
\hline
\end{tabular}


Absolute unscaled error

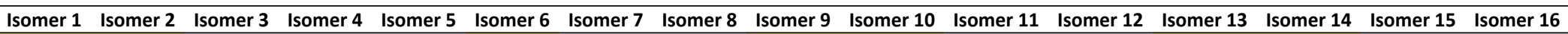

\begin{tabular}{|c|c|c|c|c|c|c|c|c|c|c|c|c|c|c|c|}
\hline & & & & & & & & & & & & & & & \\
\hline 4.2 & 3.4 & 1.8 & 1.5 & 1.2 & 3.7 & 2.6 & 2.0 & 3.4 & 4.6 & 0.6 & 0.5 & 3.0 & 3.1 & 2.2 & 2.3 \\
\hline 4.5 & 3.3 & 2.9 & 2.5 & 4.1 & 2.8 & 4.6 & 4.7 & 4.6 & 4.5 & 1.7 & 2.1 & 3.7 & 2.7 & 4.3 & 3.8 \\
\hline 15.8 & 8.0 & 7.8 & 3.9 & 11.1 & 11.3 & 5.9 & 5.3 & 16.2 & 15.8 & 3.9 & 3.4 & 10.7 & 10.6 & 1.6 & 1.8 \\
\hline 2.8 & 2.8 & 2.9 & 4.0 & 2.0 & 1.1 & 3.9 & 3.7 & 3.1 & 2.8 & 5.6 & 6.0 & 3.8 & 2.8 & 5.6 & 5.3 \\
\hline 6.9 & 6.7 & 6.0 & 2.8 & 8.2 & 11.4 & 7.1 & 7.2 & 5.4 & 7.5 & 4.3 & 10.6 & 6.7 & 10.0 & 10.1 & 14.3 \\
\hline 1.5 & 1.5 & 1.3 & 1.1 & 2.7 & 1.0 & 2.4 & 1.2 & 2.1 & 1.2 & 2.9 & 1.4 & 3.5 & 1.0 & 2.8 & 1.0 \\
\hline 0.5 & 1.3 & 1.5 & 1.0 & 1.5 & 0.0 & 2.1 & 1.5 & 1.2 & 0.2 & 4.3 & 2.3 & 1.9 & 0.3 & 2.8 & 2.5 \\
\hline 4.1 & 4.7 & 6.7 & 7.0 & 5.9 & 5.7 & 5.8 & 3.8 & 4.3 & 4.0 & 8.0 & 7.7 & 6.4 & 4.8 & 5.0 & 5.3 \\
\hline 5.1 & 3.8 & 4.7 & 3.9 & 5.5 & 4.8 & 3.4 & 1.3 & 6.8 & 4.2 & 1.8 & 1.7 & 5.5 & 4.0 & 1.2 & 1.5 \\
\hline 5.7 & 0.5 & 0.9 & 1.7 & 1.0 & 2.4 & 2.9 & 2.2 & 4.8 & 6.5 & 3.6 & 3.4 & 6.4 & 6.2 & 3.9 & 3.4 \\
\hline 1.4 & 1.9 & 2.5 & 3.2 & 0.5 & 0.4 & 3.8 & 2.9 & 1.4 & 1.5 & 1.2 & 1.9 & 1.1 & 1.8 & 1.5 & 1.7 \\
\hline 1.9 & 1.7 & 1.3 & 1.9 & 1.1 & 1.3 & 0.6 & 1.5 & 2.0 & 2.2 & 2.0 & 1.7 & 1.5 & 3.0 & 2.0 & 1.2 \\
\hline 2.6 & 2.2 & 1.2 & 1.0 & 1.6 & 1.2 & 1.7 & 1.1 & 2.9 & 2.6 & 3.3 & 3.4 & 2.0 & 1.9 & 3.1 & 3.3 \\
\hline 2.0 & 1.3 & 3.6 & 3.4 & 3.4 & 4.6 & 3.2 & 3.2 & 1.9 & 2.5 & 3.0 & 3.3 & 3.3 & 1.8 & 3.0 & 2.9 \\
\hline 1.8 & 1.3 & 1.4 & 1.3 & 1.7 & 1.2 & 1.5 & 1.4 & 2.6 & 1.5 & 2.3 & 1.1 & 1.1 & 1.1 & 2.0 & 1.7 \\
\hline 1.8 & 1.6 & 1.8 & 1.7 & 2.2 & 1.9 & 1.6 & 1.6 & 1.3 & 2.0 & 1.2 & 1.6 & 2.3 & 1.3 & 1.3 & 1.4 \\
\hline 3.1 & 2.1 & 0.7 & 3.3 & 3.0 & 4.9 & 3.2 & 3.5 & 2.6 & 3.3 & 3.4 & 3.7 & 2.6 & 4.7 & 3.4 & 3.1 \\
\hline 2.4 & 0.2 & 0.4 & 2.8 & 2.5 & 2.0 & 3.0 & 2.9 & 2.3 & 2.4 & 2.8 & 2.5 & 2.8 & 1.4 & 2.9 & 3.2 \\
\hline 1.5 & 1.0 & 0.6 & 0.8 & 0.4 & 0.3 & 2.4 & 1.7 & 1.7 & 1.4 & 1.1 & 1.7 & 0.2 & 0.1 & 3.0 & 2.8 \\
\hline 1.5 & 2.1 & 1.2 & 0.5 & 2.3 & 0.7 & 1.8 & 0.4 & 2.7 & 0.9 & 5.5 & 1.6 & 2.0 & 0.9 & 2.4 & 1.6 \\
\hline 0.4 & 0.5 & 0.7 & 0.1 & 1.5 & 0.1 & 1.4 & 0.0 & 1.5 & 0.2 & 2.0 & 0.6 & 0.8 & 0.1 & 0.8 & 0.7 \\
\hline 3.4 & 2.5 & 2.5 & 2.4 & 3.0 & 3.0 & 3.1 & 2.5 & 3.6 & 3.4 & 3.1 & 3.0 & 3.4 & 3.0 & 3.1 & 3.1 \\
\hline 0.07 & 0.11 & 0.27 & 0.23 & 0.42 & 0.26 & 0.30 & 0.15 & 0.13 & 0.04 & 0.35 & 0.30 & 0.43 & 0.16 & 0.40 & 0.25 \\
\hline 0.14 & 0.01 & 0.08 & 0.45 & 0.21 & 0.21 & 0.04 & 0.02 & 0.07 & 0.17 & 0.69 & 0.61 & 0.32 & 0.24 & 0.16 & 0.06 \\
\hline 0.36 & 0.31 & 0.46 & 0.03 & 0.45 & 0.35 & 0.46 & 0.41 & 0.40 & 0.32 & 0.03 & 0.02 & 0.36 & 0.35 & 0.44 & 0.43 \\
\hline 0.27 & 0.25 & 0.36 & 0.27 & 0.48 & 0.52 & 0.31 & 0.37 & 0.22 & 0.24 & 0.02 & 0.07 & 0.02 & 0.04 & 0.10 & 0.07 \\
\hline 0.28 & 0.27 & 0.27 & 0.17 & 0.27 & 0.25 & 0.02 & 0.15 & 0.27 & 0.26 & 0.13 & 0.03 & 0.01 & 0.03 & 0.08 & 0.06 \\
\hline 0.18 & 0.18 & 0.20 & 0.44 & 0.33 & 0.06 & 0.26 & 0.65 & 0.38 & 0.10 & 0.15 & 0.35 & 0.39 & 0.10 & 0.11 & 0.50 \\
\hline 0.03 & 0.05 & 0.02 & 0.09 & 0.13 & 0.04 & 0.03 & 0.00 & 0.08 & 0.01 & 0.05 & 0.02 & 0.19 & 0.15 & 0.00 & 0.03 \\
\hline 0.01 & 0.05 & 0.02 & 0.00 & 0.18 & 0.03 & 0.02 & 0.01 & 0.25 & 0.13 & 0.17 & 0.03 & 0.15 & 0.11 & 0.22 & 0.06 \\
\hline 0.16 & 0.04 & 0.05 & 0.10 & 0.01 & 0.03 & 0.19 & 0.13 & 0.26 & 0.12 & 0.13 & 0.00 & 0.02 & 0.12 & 0.25 & 0.07 \\
\hline 0.04 & 0.17 & 0.16 & 0.05 & 0.10 & 0.14 & 0.08 & 0.11 & 0.12 & 0.11 & 0.21 & 0.11 & 0.17 & 0.04 & 0.21 & 0.21 \\
\hline 0.07 & 0.11 & 0.31 & 0.24 & 0.25 & 0.21 & 0.09 & 0.24 & 0.40 & 0.07 & 0.38 & 0.11 & 0.31 & 0.18 & 0.43 & 0.12 \\
\hline 0.22 & 0.15 & 0.22 & 0.27 & 0.27 & 0.25 & 0.28 & 0.25 & 0.26 & 0.20 & 0.07 & 0.20 & 0.26 & 0.22 & 0.03 & 0.16 \\
\hline 0.04 & 0.01 & 0.18 & 0.40 & 0.08 & 0.07 & 0.28 & 0.37 & 0.03 & 0.04 & 0.00 & 0.04 & 0.05 & 0.02 & 0.00 & 0.04 \\
\hline 0.01 & 0.02 & 0.47 & 0.36 & 0.48 & 0.59 & 0.32 & 0.41 & 0.11 & 0.00 & 0.19 & 0.12 & 0.13 & 0.00 & 0.20 & 0.13 \\
\hline 0.11 & 0.15 & 0.09 & 0.05 & 0.04 & 0.05 & 0.03 & 0.00 & 0.07 & 0.11 & 0.05 & 0.05 & 0.08 & 0.14 & 0.04 & 0.00 \\
\hline 0.13 & 0.04 & 0.08 & 0.16 & 0.04 & 0.20 & 0.10 & 0.13 & 0.10 & 0.12 & 0.10 & 0.08 & 0.09 & 0.04 & 0.12 & 0.09 \\
\hline 0.06 & 0.07 & 0.13 & 0.17 & 0.21 & 0.23 & 0.20 & 0.21 & 0.07 & 0.04 & 0.05 & 0.12 & 0.02 & 0.11 & 0.05 & 0.10 \\
\hline 0.62 & 0.47 & 0.50 & 0.27 & 0.47 & 0.89 & 0.25 & 0.23 & 0.34 & 0.75 & 0.17 & 0.18 & 0.49 & 0.78 & 0.19 & 0.09 \\
\hline 0.04 & 0.05 & 0.06 & 0.02 & 0.02 & 0.11 & 0.00 & 0.01 & 0.05 & 0.03 & 0.00 & 0.01 & 0.02 & 0.08 & 0.02 & 0.00 \\
\hline 0.14 & 0.07 & 0.04 & 0.08 & 0.13 & 0.17 & 0.10 & 0.09 & 0.12 & 0.16 & 0.14 & 0.11 & 0.16 & 0.20 & 0.13 & 0.12 \\
\hline 0.01 & 0.00 & 0.06 & 0.15 & 0.09 & 0.04 & 0.08 & 0.01 & 0.03 & 0.00 & 0.11 & 0.11 & 0.12 & 0.02 & 0.12 & 0.02 \\
\hline 0.04 & 0.02 & 0.01 & 0.03 & 0.07 & 0.03 & 0.07 & 0.05 & 0.08 & 0.03 & 0.01 & 0.02 & 0.17 & 0.03 & 0.01 & 0.03 \\
\hline 0.04 & 0.02 & 0.04 & 0.01 & 0.09 & 0.07 & 0.02 & 0.05 & 0.02 & 0.04 & 0.12 & 0.12 & 0.04 & 0.02 & 0.09 & 0.15 \\
\hline 0.13 & 0.11 & 0.18 & 0.18 & 0.21 & 0.21 & 0.15 & 0.18 & 0.17 & 0.13 & 0.14 & 0.12 & 0.17 & 0.14 & 0.15 & 0.12 \\
\hline
\end{tabular}


Absolute unscaled error

\begin{tabular}{|c|c|c|c|c|c|c|c|c|c|c|c|c|c|c|c|c|}
\hline & Isomer 17 & Isomer 18 & Isomer 19 & Isomer 20 & Isomer 21 & Isomer 22 & Isomer 23 & Isomer 24 & Isomer 25 & Isomer 26 & Isomer 27 & Isomer 28 & Isomer 29 & Isomer 30 & Isomer 31 & Isomer 32 \\
\hline C 1 & $4 \quad 4.0$ & $4 \quad 4.2$ & $\begin{array}{l}0.6 \\
\end{array}$ & 0.4 & 3 & 3.4 .4 & 101.7 & 101.6 & $4 \quad 4.1$ & 2.6 & $\begin{array}{l}1.2 \\
\end{array}$ & "1.0 & 3.5 & 2.7 & 3.0 & $\begin{array}{l}4.0 \\
\end{array}$ \\
\hline$C 2$ & 3.4 & 3.8 & 2.0 & 2.2 & 2.4 & 2.1 & 4.4 & 4.9 & 3.5 & 4.7 & 2.9 & 3.0 & 4.5 & 5.0 & 5.0 & 5.4 \\
\hline C 3 & 2.5 & 2.7 & 9.9 & 9.9 & 3.1 & 3.8 & 12.6 & 11.9 & 10.0 & 7.3 & 9.7 & 9.2 & 4.9 & 5.6 & 11.6 & 11.4 \\
\hline C4 & 3.5 & 2.5 & 5.1 & 4.2 & 3.5 & 3.3 & 4.0 & 4.1 & 1.2 & 1.8 & 3.0 & 2.8 & 2.3 & 3.1 & 2.9 & 2.7 \\
\hline C 5 & 1.8 & 8.8 & 4.0 & 10.6 & 5.2 & 7.0 & 8.5 & 14.9 & 1.8 & 5.8 & 4.3 & 5.3 & 6.0 & 6.9 & 8.5 & 9.9 \\
\hline C 6 & 2.4 & 0.4 & 2.3 & 1.8 & 2.4 & 1.0 & 2.1 & 1.0 & 2.5 & 1.7 & 2.2 & 0.4 & 2.3 & 1.0 & 2.2 & 0.3 \\
\hline C 7 & 4.1 & 0.2 & 4.2 & 2.1 & 3.1 & 2.3 & 3.3 & 1.8 & 3.5 & 1.7 & 3.1 & 2.5 & 2.9 & 2.6 & 2.8 & 1.6 \\
\hline C 8 & 3.7 & 4.7 & 8.4 & 7.3 & 6.9 & 4.7 & 5.2 & 5.3 & 5.7 & 6.3 & 8.3 & 6.8 & 6.4 & 4.5 & 6.0 & 3.7 \\
\hline C9 & 4.6 & 3.9 & 3.1 & 0.3 & 4.4 & 2.9 & 2.3 & 0.9 & 3.2 & 3.6 & 5.8 & 5.2 & 4.5 & 4.2 & 4.5 & 4.3 \\
\hline C 10 & 4.8 & 3.8 & 5.4 & 5.2 & 5.4 & 3.0 & 3.9 & 4.9 & 1.9 & 0.8 & 2.1 & 4.2 & 0.9 & 0.4 & 2.0 & 4.0 \\
\hline C 11 & 2.7 & 2.4 & 4.1 & 3.3 & 4.7 & 3.7 & 3.5 & 4.1 & 1.3 & 4.6 & 4.0 & 3.2 & 3.7 & 4.0 & 3.7 & 2.8 \\
\hline C 12 & 1.7 & 2.1 & 2.8 & 2.9 & 2.7 & 2.5 & 2.7 & 1.8 & 0.5 & 0.7 & 1.1 & 1.1 & 0.1 & 0.3 & 0.9 & 0.9 \\
\hline C 13 & 2.6 & 2.6 & 3.8 & 3.6 & 2.4 & 2.4 & 3.5 & 3.0 & 1.6 & 1.6 & 1.7 & 0.7 & 1.2 & 1.9 & 1.7 & 0.9 \\
\hline C 14 & 3.1 & 2.5 & 2.8 & 3.7 & 3.5 & 3.1 & 3.4 & 3.2 & 3.3 & 3.2 & 3.1 & 3.4 & 2.9 & 2.9 & 4.1 & 3.6 \\
\hline C 15 & 1.2 & 1.3 & 1.9 & 2.0 & 1.7 & 1.4 & 1.6 & 1.7 & 1.5 & 1.9 & 1.4 & 1.3 & 1.7 & 1.8 & 2.0 & 2.2 \\
\hline C 16 & 1.2 & 2.2 & 1.9 & 1.6 & 1.4 & 1.6 & 1.8 & 1.6 & 1.5 & 1.9 & 1.8 & 1.6 & 1.8 & 1.9 & 2.3 & 1.9 \\
\hline C 17 & 0.5 & 0.5 & 0.2 & 0.2 & 0.6 & 0.7 & 0.2 & 0.2 & 0.3 & 0.1 & 0.2 & 0.3 & 0.3 & 0.4 & 0.2 & 0.1 \\
\hline C 18 & 0.8 & 0.9 & 0.4 & 0.1 & 0.6 & 0.8 & 0.4 & 0.4 & 0.9 & 0.5 & 0.4 & 0.4 & 0.4 & 1.0 & 0.5 & 0.4 \\
\hline C 19 & 1.9 & 1.1 & 0.6 & 1.4 & 0.7 & 0.7 & 3.9 & 3.4 & 1.7 & 0.1 & 0.4 & 0.2 & 1.5 & 1.3 & 2.8 & 2.0 \\
\hline$C 20$ & 4.3 & 1.5 & 3.9 & 1.1 & 2.2 & 0.4 & 1.9 & 1.5 & 4.2 & 1.0 & 3.6 & 0.6 & 2.6 & 0.8 & 2.7 & 0.3 \\
\hline $\mathrm{COCH}_{3}$ & 1.6 & 0.7 & 2.7 & 0.7 & 0.9 & 0.3 & 1.6 & 1.0 & 1.9 & 0.2 & 2.4 & 0.3 & 1.1 & 0.4 & 1.3 & 0.1 \\
\hline Average & 2.7 & 2.5 & 3.3 & 3.1 & 2.9 & 2.4 & 3.5 & 3.5 & 2.7 & 2.5 & 3.0 & 2.5 & 2.6 & 2.5 & 3.4 & 3.0 \\
\hline H 1 & 0.15 & 0.02 & 0.28 & 0.23 & 0.36 & 0.24 & 0.39 & 0.28 & 0.11 & 0.08 & 0.34 & 0.25 & 0.34 & 0.25 & 0.34 & 0.10 \\
\hline $\mathrm{H} 2 \mathrm{a}$ & 0.14 & 0.15 & 0.78 & 0.80 & 0.14 & 0.09 & 0.25 & 0.24 & 0.11 & 0.03 & 0.52 & 0.50 & 0.12 & 0.06 & 0.06 & 0.01 \\
\hline $\mathrm{H} 2 \mathrm{~b}$ & 0.19 & 0.15 & 0.10 & 0.07 & 0.37 & 0.31 & 0.61 & 0.54 & 0.19 & 0.20 & 0.14 & 0.08 & 0.29 & 0.27 & 0.53 & 0.43 \\
\hline H 3a & 0.05 & 0.02 & 0.10 & 0.09 & 0.20 & 0.24 & 0.08 & 0.01 & 0.62 & 0.14 & 0.41 & 0.44 & 0.12 & 0.23 & 0.35 & 0.30 \\
\hline$H 3 b$ & 0.08 & 0.03 & 0.21 & 0.18 & 0.29 & 0.28 & 0.20 & 0.21 & 0.12 & 0.41 & 0.21 & 0.16 & 0.59 & 0.53 & 0.25 & 0.11 \\
\hline H 5 & 0.60 & 0.20 & 0.14 & 0.29 & 0.49 & 0.15 & 0.11 & 0.33 & 0.58 & 0.05 & 0.18 & 0.46 & 0.33 & 0.13 & 0.31 & 0.59 \\
\hline $\mathrm{H} 7 \mathrm{a}$ & 0.05 & 0.06 & 0.07 & 0.00 & 0.05 & 0.03 & 0.10 & 0.02 & 0.06 & 0.02 & 0.03 & 0.00 & 0.01 & 0.02 & 0.01 & 0.02 \\
\hline$H 7 b$ & 0.07 & 0.05 & 0.15 & 0.04 & 0.04 & 0.02 & 0.12 & 0.00 & 0.08 & 0.04 & 0.13 & 0.01 & 0.15 & 0.03 & 0.09 & 0.00 \\
\hline H $8 \mathrm{a}$ & 0.30 & 0.09 & 0.05 & 0.10 & 0.00 & 0.00 & 0.10 & 0.06 & 0.27 & 0.03 & 0.11 & 0.06 & 0.09 & 0.04 & 0.19 & 0.07 \\
\hline H 8b & 0.10 & 0.14 & 0.19 & 0.13 & 0.18 & 0.05 & 0.05 & 0.23 & 0.09 & 0.21 & 0.13 & 0.18 & 0.13 & 0.09 & 0.08 & 0.18 \\
\hline H 10 & 0.21 & 0.17 & 0.28 & 0.79 & 0.14 & 0.20 & 0.31 & 0.77 & 0.06 & 0.43 & 0.60 & 0.83 & 0.09 & 0.46 & 0.38 & 0.75 \\
\hline H 11 & 0.07 & 0.02 & 0.19 & 0.21 & 0.07 & 0.02 & 0.19 & 0.30 & 0.24 & 0.01 & 0.10 & 0.09 & 0.03 & 0.05 & 0.03 & 0.03 \\
\hline H $12 a-c$ & 0.03 & 0.00 & 0.06 & 0.07 & 0.00 & 0.02 & 0.04 & 0.10 & 0.32 & 0.32 & 0.05 & 0.03 & 0.28 & 0.33 & 0.04 & 0.04 \\
\hline H 13a & 0.11 & 0.09 & 0.02 & 0.13 & 0.09 & 0.08 & 0.07 & 0.14 & 0.42 & 0.37 & 0.55 & 0.78 & 0.35 & 0.33 & 0.48 & 0.71 \\
\hline H 13b & 0.04 & 0.03 & 0.13 & 0.10 & 0.01 & 0.05 & 0.13 & 0.10 & 0.05 & 0.03 & 0.08 & 0.02 & 0.08 & 0.07 & 0.04 & 0.00 \\
\hline H 14a & 0.04 & 0.05 & 0.01 & 0.03 & 0.03 & 0.03 & 0.04 & 0.03 & 0.23 & 0.09 & 0.09 & 0.07 & 0.15 & 0.17 & 0.08 & 0.08 \\
\hline H 14b & 0.09 & 0.04 & 0.09 & 0.09 & 0.05 & 0.08 & 0.13 & 0.04 & 0.19 & 0.22 & 0.13 & 0.12 & 0.18 & 0.18 & 0.16 & 0.09 \\
\hline H 15 & 0.13 & 0.11 & 0.47 & 0.57 & 0.22 & 0.18 & 0.51 & 0.54 & 0.20 & 0.22 & 0.47 & 0.56 & 0.25 & 0.28 & 0.48 & 0.49 \\
\hline H $17 a-c$ & 0.05 & 0.08 & 0.03 & 0.05 & 0.05 & 0.08 & 0.01 & 0.05 & 0.05 & 0.02 & 0.05 & 0.06 & 0.06 & 0.07 & 0.02 & 0.02 \\
\hline H 18a-c & 0.06 & 0.08 & 0.04 & 0.03 & 0.05 & 0.07 & 0.00 & 0.05 & 0.05 & 0.11 & 0.06 & 0.06 & 0.06 & 0.08 & 0.01 & 0.05 \\
\hline H 19a-c & 0.02 & 0.02 & 0.12 & 0.10 & 0.08 & 0.04 & 0.05 & 0.03 & 0.01 & 0.06 & 0.08 & 0.12 & 0.05 & 0.01 & 0.08 & 0.05 \\
\hline $\mathrm{H} 20 \mathrm{a}-\mathrm{c}$ & 0.00 & 0.01 & 0.01 & 0.03 & 0.00 & 0.00 & 0.03 & 0.05 & 0.02 & 0.02 & 0.02 & 0.01 & 0.01 & 0.05 & 0.03 & 0.02 \\
\hline
\end{tabular}


Absolute scaled error

\begin{tabular}{|c|c|c|c|c|c|c|c|c|c|c|c|c|c|c|c|c|}
\hline & \begin{tabular}{|l|} 
Isomer 1 \\
\end{tabular} & Isomer 2 & Isomer 3 & Isomer 4 & Isomer 5 & Isomer 6 & Isomer 7 & Isomer 8 & Isomer 9 & Isomer 10 & Isomer 11 & Isomer 12 & Isomer 13 & Isomer 14 & Isomer 15 & Isomer 16 \\
\hline C 1 & 101.6 & $\begin{array}{ll}1.1 \\
\end{array}$ & 0.3 & 0.3 & 0.7 & 10 & 0.8 & 0.5 & 0.8 & $\begin{array}{l}2.0 \\
\end{array}$ & $\begin{array}{l}0.9 \\
\end{array}$ & $\begin{array}{l}1.4 \\
\end{array}$ & $\begin{array}{l}0.7 \\
\end{array}$ & $\begin{array}{l}0.7 \\
\end{array}$ & $\begin{array}{ll}0.1 \\
\end{array}$ & $\begin{array}{ll}0.2 \\
\end{array}$ \\
\hline C 2 & 1.7 & 1.0 & 1.4 & 1.4 & 2.2 & 0.6 & 2.8 & 3.2 & 1.8 & 1.7 & 0.3 & 0.3 & 1.4 & 0.1 & 2.0 & 1.7 \\
\hline C 3 & 13.3 & 5.7 & 6.3 & 2.9 & 9.3 & 9.2 & 4.1 & 3.8 & 13.7 & 13.2 & 5.0 & 5.3 & 8.4 & 8.1 & 3.8 & 3.9 \\
\hline C 4 & 1.5 & 1.3 & 1.4 & 2.3 & 0.3 & 0.0 & 2.2 & 2.2 & 1.5 & 1.6 & 2.8 & 3.8 & 1.6 & 1.7 & 3.0 & 3.5 \\
\hline C 5 & 4.2 & 4.5 & 4.5 & 1.7 & 6.4 & 9.5 & 5.3 & 5.7 & 2.7 & 4.9 & 2.8 & 8.7 & 4.4 & 7.7 & 7.8 & 12.3 \\
\hline C 6 & 0.4 & 0.1 & 0.1 & 0.7 & 1.0 & 0.2 & 0.6 & 0.3 & 0.7 & 0.2 & 0.1 & 0.8 & 1.2 & 0.1 & 0.1 & 0.8 \\
\hline C 7 & 2.1 & 0.9 & 0.0 & 0.2 & 0.4 & 2.0 & 0.3 & 0.0 & 1.4 & 2.5 & 2.6 & 4.3 & 0.4 & 2.0 & 0.4 & 4.6 \\
\hline C 8 & 1.4 & 2.4 & 5.2 & 5.8 & 4.0 & 3.6 & 4.0 & 2.4 & 1.6 & 1.2 & 6.3 & 5.8 & 4.1 & 2.3 & 2.7 & 3.3 \\
\hline C9 & 2.6 & 1.6 & 3.2 & 2.7 & 3.7 & 2.9 & 1.6 & 0.2 & 4.3 & 1.7 & 0.2 & 0.3 & 3.2 & 1.7 & 1.1 & 3.5 \\
\hline C 10 & 8.6 & 2.8 & 2.4 & 0.5 & 2.9 & 4.6 & 1.1 & 0.7 & 7.6 & 9.5 & 2.0 & 1.4 & 8.7 & 8.8 & 1.6 & 1.4 \\
\hline C 11 & 1.0 & 0.2 & 4.0 & 4.5 & 2.3 & 2.2 & 5.5 & 4.4 & 1.0 & 0.9 & 0.6 & 0.1 & 1.1 & 0.4 & 0.8 & 0.3 \\
\hline C 12 & 1.2 & 0.8 & 2.8 & 2.8 & 3.1 & 3.7 & 2.4 & 2.9 & 1.0 & 1.0 & 0.9 & 0.1 & 0.8 & 0.2 & 0.2 & 0.9 \\
\hline C 13 & 0.1 & 0.1 & 0.3 & 0.1 & 0.3 & 1.0 & 0.1 & 0.4 & 0.2 & 0.2 & 1.8 & 1.4 & 0.3 & 0.7 & 0.8 & 1.3 \\
\hline C 14 & 0.8 & 1.1 & 5.1 & 4.4 & 5.3 & 6.9 & 5.0 & 4.6 & 0.9 & 0.4 & 1.6 & 1.4 & 1.0 & 0.8 & 0.7 & 0.8 \\
\hline C 15 & 0.5 & 0.3 & 0.1 & 0.3 & 0.1 & 0.1 & 0.2 & 0.1 & 1.0 & 0.3 & 0.5 & 1.1 & 1.1 & 0.0 & 0.6 & 0.1 \\
\hline C 16 & 0.3 & 0.0 & 0.3 & 0.1 & 0.4 & 0.9 & 0.1 & 0.1 & 0.4 & 0.6 & 1.4 & 0.6 & 0.1 & 0.1 & 1.3 & 0.5 \\
\hline C 17 & 0.3 & 0.3 & 0.8 & 2.2 & 1.1 & 2.8 & 1.4 & 2.0 & 0.2 & 0.4 & 2.0 & 1.8 & 0.3 & 2.2 & 1.1 & 1.1 \\
\hline C 18 & 5.3 & 2.6 & 1.1 & 3.9 & 4.4 & 4.2 & 4.8 & 4.4 & 5.2 & 5.4 & 4.1 & 4.4 & 5.1 & 4.0 & 5.2 & 5.2 \\
\hline C 19 & 1.6 & 1.5 & 0.9 & 0.2 & 1.5 & 2.1 & 0.6 & 0.3 & 1.3 & 1.8 & 0.1 & 0.2 & 2.1 & 2.9 & 0.8 & 0.8 \\
\hline C 20 & 4.5 & 4.5 & 2.7 & 1.6 & 4.2 & 3.0 & 3.6 & 1.9 & 5.6 & 4.0 & 6.6 & 3.4 & 4.3 & 3.6 & 4.6 & 3.6 \\
\hline $\mathrm{COCH}_{3}$ & 2.5 & 2.5 & 2.1 & 1.4 & 3.3 & 1.7 & 3.1 & 1.5 & 3.7 & 2.0 & 4.0 & 2.6 & 3.0 & 1.8 & 3.2 & 2.7 \\
\hline Average & 2.6 & 1.7 & 2.2 & 1.9 & 2.7 & 3.0 & 2.4 & 2.0 & 2.7 & 2.6 & 2.2 & 2.3 & 2.5 & 2.4 & 2.0 & 2.5 \\
\hline H 1 & 0.02 & 0.05 & 0.20 & 0.16 & 0.35 & 0.14 & 0.23 & 0.11 & 0.10 & 0.03 & 0.31 & 0.26 & 0.34 & 0.06 & 0.36 & 0.25 \\
\hline $\mathrm{H} 2 \mathrm{a}$ & 0.16 & 0.07 & 0.01 & 0.38 & 0.14 & 0.11 & 0.10 & 0.03 & 0.09 & 0.19 & 0.64 & 0.57 & 0.24 & 0.16 & 0.13 & 0.03 \\
\hline $\mathrm{H} 2 \mathrm{~b}$ & 0.34 & 0.26 & 0.39 & 0.01 & 0.38 & 0.29 & 0.40 & 0.37 & 0.39 & 0.31 & 0.02 & 0.00 & 0.32 & 0.31 & 0.41 & 0.39 \\
\hline H 3a & 0.31 & 0.31 & 0.44 & 0.19 & 0.55 & 0.59 & 0.24 & 0.33 & 0.25 & 0.29 & 0.02 & 0.11 & 0.07 & 0.14 & 0.06 & 0.06 \\
\hline$H 3 b$ & 0.26 & 0.22 & 0.20 & 0.18 & 0.20 & 0.19 & 0.07 & 0.20 & 0.26 & 0.24 & 0.11 & 0.00 & 0.04 & 0.00 & 0.05 & 0.01 \\
\hline H 5 & 0.14 & 0.12 & 0.28 & 0.47 & 0.26 & 0.03 & 0.31 & 0.70 & 0.36 & 0.06 & 0.11 & 0.38 & 0.31 & 0.02 & 0.14 & 0.56 \\
\hline $\mathrm{H} 7 \mathrm{a}$ & 0.01 & 0.01 & 0.10 & 0.04 & 0.06 & 0.05 & 0.03 & 0.04 & 0.05 & 0.03 & 0.08 & 0.01 & 0.11 & 0.06 & 0.04 & 0.01 \\
\hline $\mathrm{H} 7 \mathrm{~b}$ & 0.03 & 0.00 & 0.06 & 0.04 & 0.25 & 0.11 & 0.08 & 0.03 & 0.26 & 0.08 & 0.20 & 0.06 & 0.21 & 0.03 & 0.25 & 0.09 \\
\hline $\mathrm{H} 8 \mathrm{a}$ & 0.10 & 0.11 & 0.13 & 0.16 & 0.06 & 0.08 & 0.12 & 0.17 & 0.23 & 0.04 & 0.09 & 0.03 & 0.07 & 0.22 & 0.22 & 0.08 \\
\hline $\mathrm{H} 8 \mathrm{~b}$ & 0.00 & 0.10 & 0.08 & 0.00 & 0.03 & 0.04 & 0.01 & 0.07 & 0.14 & 0.05 & 0.17 & 0.08 & 0.09 & 0.05 & 0.17 & 0.20 \\
\hline $\mathrm{H} 10$ & 0.08 & 0.06 & 0.24 & 0.20 & 0.32 & 0.14 & 0.15 & 0.20 & 0.40 & 0.05 & 0.39 & 0.08 & 0.34 & 0.12 & 0.46 & 0.08 \\
\hline H 11 & 0.26 & 0.21 & 0.29 & 0.31 & 0.34 & 0.33 & 0.34 & 0.30 & 0.28 & 0.24 & 0.04 & 0.17 & 0.32 & 0.29 & 0.01 & 0.16 \\
\hline H 12a-c & 0.04 & 0.03 & 0.11 & 0.36 & 0.01 & 0.02 & 0.22 & 0.33 & 0.04 & 0.04 & 0.01 & 0.01 & 0.08 & 0.01 & 0.03 & 0.02 \\
\hline H 13a & 0.06 & 0.04 & 0.39 & 0.30 & 0.41 & 0.45 & 0.25 & 0.37 & 0.13 & 0.05 & 0.22 & 0.15 & 0.19 & 0.09 & 0.23 & 0.16 \\
\hline H 13b & 0.07 & 0.09 & 0.02 & 0.01 & 0.03 & 0.03 & 0.03 & 0.05 & 0.05 & 0.06 & 0.02 & 0.02 & 0.02 & 0.07 & 0.00 & 0.04 \\
\hline H 14a & 0.17 & 0.10 & 0.01 & 0.10 & 0.03 & 0.09 & 0.03 & 0.08 & 0.12 & 0.16 & 0.14 & 0.11 & 0.15 & 0.12 & 0.15 & 0.11 \\
\hline H 14b & 0.03 & 0.01 & 0.21 & 0.20 & 0.28 & 0.29 & 0.26 & 0.25 & 0.05 & 0.01 & 0.02 & 0.08 & 0.04 & 0.03 & 0.02 & 0.07 \\
\hline H 15 & 0.45 & 0.36 & 0.42 & 0.13 & 0.40 & 0.60 & 0.16 & 0.19 & 0.28 & 0.51 & 0.10 & 0.13 & 0.32 & 0.49 & 0.14 & 0.16 \\
\hline H $17 a-c$ & 0.02 & 0.10 & 0.13 & 0.04 & 0.05 & 0.04 & 0.06 & 0.04 & 0.04 & 0.02 & 0.03 & 0.04 & 0.03 & 0.04 & 0.01 & 0.04 \\
\hline H 18a-c & 0.16 & 0.12 & 0.11 & 0.11 & 0.20 & 0.22 & 0.16 & 0.13 & 0.13 & 0.17 & 0.16 & 0.14 & 0.20 & 0.23 & 0.16 & 0.17 \\
\hline H 19a-c & 0.00 & 0.05 & 0.13 & 0.17 & 0.02 & 0.00 & 0.02 & 0.04 & 0.02 & 0.00 & 0.12 & 0.14 & 0.08 & 0.01 & 0.09 & 0.03 \\
\hline
\end{tabular}




\begin{tabular}{|c|c|c|c|c|c|c|c|c|c|c|c|c|c|c|c|c|}
\hline $\mathrm{H} \mathrm{OCH}_{3}$ & 0.18 & 0.11 & 0.12 & 0.13 & 0.16 & 0.29 & 0.11 & 0.08 & 0.07 & 0.23 & 0.19 & 0.17 & 0.20 & 0.22 & 0.13 & 0.08 \\
\hline erage & 0.13 & 0.11 & 0.18 & 0.16 & 0.20 & 0.18 & 0.15 & 0.18 & 0.16 & 0.12 & 0.14 & 0.12 & 0.17 & 0.12 & 011 & \\
\hline
\end{tabular}

Absolute scaled error

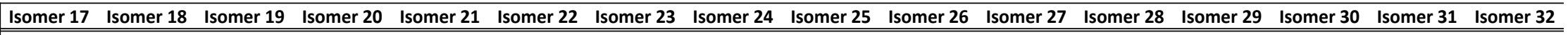

\begin{tabular}{|c|c|c|c|c|c|c|c|c|c|c|c|c|c|c|c|c|}
\hline & & & & & & & & & & & & & & & & \\
\hline C 1 & 1.9 & 1.8 & 1.6 & 1.9 & 1.6 & 1.3 & 1.3 & 1.2 & 2.5 & 1.0 & 0.2 & 0.4 & 1.8 & 0.9 & 1.0 & 2.1 \\
\hline C 2 & 1.3 & 1.3 & 0.2 & 0.2 & 0.1 & 0.0 & 1.4 & 2.1 & 1.8 & 3.1 & 1.5 & 1.6 & 2.9 & 3.3 & 3.0 & 3.4 \\
\hline C 3 & 0.4 & 0.1 & 7.7 & 7.6 & 5.4 & 5.8 & 9.7 & 9.1 & 8.4 & 5.7 & 8.2 & 7.9 & 3.2 & 3.9 & 9.6 & 9.4 \\
\hline C 4 & 1.4 & 1.0 & 2.8 & 2.6 & 1.0 & 1.3 & 2.3 & 2.8 & 0.1 & 0.4 & 1.5 & 1.7 & 0.6 & 1.5 & 1.2 & 1.5 \\
\hline C 5 & 0.4 & 6.5 & 1.8 & 8.4 & 2.9 & 5.0 & 5.7 & 12.4 & 0.1 & 4.2 & 2.9 & 3.9 & 4.4 & 5.2 & 6.5 & 8.0 \\
\hline C 7 & 2.0 & 2.6 & 2.0 & 4.4 & 0.8 & 0.2 & 0.5 & 4.5 & 1.9 & 0.1 & 1.6 & 1.2 & 1.3 & 0.8 & 0.9 & 0.3 \\
\hline C 8 & 1.6 & 2.3 & 6.2 & 5.0 & 4.6 & 2.7 & 2.3 & 2.5 & 4.0 & 4.8 & 6.9 & 5.4 & 4.7 & 2.8 & 4.0 & 1.7 \\
\hline C9 & 2.5 & 1.6 & 0.9 & 1.9 & 2.1 & 0.9 & 0.6 & 3.6 & 1.6 & 2.0 & 4.4 & 3.8 & 2.8 & 2.5 & 2.5 & 2.4 \\
\hline C 10 & 2.7 & 1.4 & 7.6 & 7.6 & 3.1 & 1.0 & 7.1 & 7.9 & 0.3 & 0.8 & 3.6 & 5.6 & 0.8 & 2.1 & 4.1 & 6.1 \\
\hline C 11 & 0.6 & 0.2 & 1.9 & 1.2 & 2.3 & 1.7 & 0.9 & 1.7 & 2.9 & 6.1 & 5.4 & 4.6 & 5.4 & 5.7 & 5.7 & 4.7 \\
\hline C 13 & 0.5 & 0.1 & 1.6 & 1.3 & 0.1 & 0.4 & 0.5 & 0.2 & 0.1 & 0.0 & 0.3 & 0.7 & 0.5 & 0.1 & 0.4 & 1.2 \\
\hline C 14 & 1.0 & 0.0 & 0.7 & 1.3 & 1.3 & 1.1 & 0.3 & 0.3 & 5.0 & 4.8 & 4.5 & 4.9 & 4.6 & 4.7 & 6.2 & 5.8 \\
\hline C 15 & 0.9 & 0.2 & 0.3 & 0.3 & 0.8 & 0.7 & 0.1 & 0.4 & 0.2 & 0.5 & 0.0 & 0.2 & 0.0 & 0.3 & 0.3 & 1.0 \\
\hline C 16 & 0.9 & 0.7 & 0.4 & 0.1 & 1.1 & 0.4 & 0.1 & 0.2 & 0.1 & 0.5 & 0.3 & 0.5 & 0.2 & 0.3 & 0.6 & 0.6 \\
\hline C 17 & 1.6 & 2.2 & 1.9 & 2.2 & 1.6 & 1.3 & 3.0 & 2.9 & 1.4 & 1.5 & 1.2 & 1.1 & 1.3 & 1.4 & 1.8 & 2.1 \\
\hline C 18 & 1.3 & 1.7 & 1.7 & 2.3 & 1.7 & 1.2 & 2.7 & 2.6 & 0.8 & 1.1 & 1.0 & 1.0 & 1.2 & 0.8 & 1.5 & 1.7 \\
\hline C 19 & 0.2 & 1.6 & 1.6 & 1.1 & 1.6 & 1.3 & 0.7 & 0.3 & 0.0 & 1.7 & 1.0 & 1.2 & 0.1 & 0.5 & 0.7 & 0.2 \\
\hline C 20 & 6.5 & 4.2 & 6.0 & 3.5 & 4.5 & 2.4 & 5.1 & 4.6 & 5.9 & 2.7 & 5.0 & 2.0 & 4.3 & 2.5 & 4.8 & 2.4 \\
\hline $\mathrm{OCH}_{3}$ & 3.8 & 2.7 & 4.9 & 2.8 & 3.2 & 1.7 & 4.1 & 3.2 & 3.4 & 1.8 & 3.9 & 1.5 & 2.7 & 1.3 & 3.2 & 1.6 \\
\hline rage & 1.5 & 1.6 & 2.5 & 2.7 & 1.9 & 1.5 & 2.4 & 3.0 & 2.1 & 2.2 & 2.7 & 2.5 & 2.2 & 2.1 & 2.9 & 2.9 \\
\hline H 2a & 0.16 & 0.18 & 0.68 & 0.69 & 0.07 & 0.04 & 0.16 & 0.15 & 0.18 & 0.12 & 0.42 & 0.40 & 0.00 & 0.04 & 0.02 & 0.09 \\
\hline $\mathrm{H} 2 \mathrm{~b}$ & 0.18 & 0.11 & 0.02 & 0.01 & 0.30 & 0.25 & 0.52 & 0.44 & 0.13 & 0.09 & 0.07 & 0.03 & 0.15 & 0.16 & 0.45 & 0.36 \\
\hline H 3a & 0.00 & 0.00 & 0.00 & 0.19 & 0.26 & 0.29 & 0.17 & 0.10 & 0.49 & 0.21 & 0.50 & 0.51 & 0.23 & 0.32 & 0.43 & 0.37 \\
\hline H 3b & 0.06 & 0.01 & 0.13 & 0.09 & 0.21 & 0.22 & 0.11 & 0.10 & 0.17 & 0.33 & 0.14 & 0.09 & 0.47 & 0.43 & 0.17 & 0.04 \\
\hline H 5 & 0.56 & 0.18 & 0.05 & 0.37 & 0.43 & 0.09 & 0.20 & 0.45 & 0.48 & 0.14 & 0.26 & 0.51 & 0.22 & 0.24 & 0.39 & 0.65 \\
\hline H 7a & 0.08 & 0.04 & 0.16 & 0.09 & 0.02 & 0.09 & 0.19 & 0.08 & 0.14 & 0.06 & 0.11 & 0.09 & 0.11 & 0.12 & 0.08 & 0.06 \\
\hline$H 7 b$ & 0.10 & 0.03 & 0.24 & 0.05 & 0.11 & 0.07 & 0.21 & 0.10 & 0.15 & 0.04 & 0.21 & 0.09 & 0.27 & 0.07 & 0.17 & 0.07 \\
\hline H 8a & 0.25 & 0.08 & 0.05 & 0.20 & 0.06 & 0.05 & 0.01 & 0.15 & 0.16 & 0.09 & 0.01 & 0.16 & 0.01 & 0.13 & 0.10 & 0.15 \\
\hline $\mathrm{H} 8 \mathrm{~b}$ & 0.13 & 0.12 & 0.10 & 0.03 & 0.12 & 0.00 & 0.04 & 0.14 & 0.17 & 0.15 & 0.04 & 0.09 & 0.02 & 0.00 & 0.01 & 0.10 \\
\hline H 10 & 0.23 & 0.14 & 0.19 & 0.69 & 0.22 & 0.15 & 0.21 & 0.69 & 0.12 & 0.36 & 0.51 & 0.73 & 0.04 & 0.36 & 0.29 & 0.67 \\
\hline H 11 & 0.11 & 0.04 & 0.29 & 0.31 & 0.14 & 0.07 & 0.28 & 0.40 & 0.32 & 0.09 & 0.19 & 0.18 & 0.08 & 0.04 & 0.12 & 0.10 \\
\hline$+12 a-c$ & 0.04 & 0.04 & 0.02 & 0.01 & 0.09 & 0.04 & 0.05 & 0.02 & 0.25 & 0.23 & 0.02 & 0.02 & 0.14 & 0.22 & 0.04 & 0.03 \\
\hline H 13a & 0.14 & 0.12 & 0.08 & 0.04 & 0.16 & 0.13 & 0.02 & 0.04 & 0.31 & 0.31 & 0.45 & 0.66 & 0.25 & 0.25 & 0.39 & 0.62 \\
\hline $\mathrm{H} 13 \mathrm{~b}$ & 0.02 & 0.01 & 0.04 & 0.01 & 0.08 & 0.00 & 0.04 & 0.00 & 0.03 & 0.05 & 0.01 & 0.06 & 0.04 & 0.03 & 0.04 & 0.07 \\
\hline H 14a & 0.07 & 0.08 & 0.11 & 0.12 & 0.09 & 0.08 & 0.13 & 0.13 & 0.14 & 0.02 & 0.00 & 0.02 & 0.04 & 0.08 & 0.01 & 0.01 \\
\hline $\mathrm{H} 14 \mathrm{~b}$ & 0.06 & 0.01 & 0.00 & 0.00 & 0.02 & 0.03 & 0.04 & 0.06 & 0.26 & 0.31 & 0.20 & 0.19 & 0.31 & 0.29 & 0.25 & 0.16 \\
\hline H 15 & 0.05 & 0.12 & 0.33 & 0.44 & 0.20 & 0.16 & 0.42 & 0.51 & 0.03 & 0.22 & 0.33 & 0.36 & 0.23 & 0.24 & 0.37 & 0.38 \\
\hline $17 \mathrm{a}-\mathrm{c}$ & 0.07 & 0.11 & 0.12 & 0.13 & 0.13 & 0.14 & 0.11 & 0.17 & 0.12 & 0.12 & 0.13 & 0.12 & 0.20 & 0.18 & 0.10 & 0.09 \\
\hline $18 a-c$ & 0.08 & 0.11 & 0.12 & 0.12 & 0.13 & 0.13 & 0.09 & 0.17 & 0.11 & 0.21 & 0.14 & 0.12 & 0.19 & 0.19 & 0.09 & 0.12 \\
\hline
\end{tabular}




\begin{tabular}{|c|c|c|c|c|c|c|c|c|c|c|c|c|c|c|c|c|}
\hline H 19a-c & 0.01 & 0.02 & 0.19 & 0.18 & 0.00 & 0.02 & 0.04 & 0.08 & 0.04 & 0.16 & 0.15 & 0.17 & 0.09 & 0.12 & 0.00 & 0.02 \\
\hline H 20a-c & 0.02 & 0.04 & 0.08 & 0.11 & 0.07 & 0.06 & 0.13 & 0.16 & 0.05 & 0.07 & 0.10 & 0.08 & 0.12 & 0.15 & 0.11 & 0.09 \\
\hline $\mathrm{H} \mathrm{OCH}_{3}$ & 0.05 & 0.11 & 0.25 & 0.24 & 0.06 & 0.05 & 0.14 & 0.20 & 0.14 & 0.05 & 0.20 & 0.19 & 0.09 & 0.00 & 0.14 & 0.12 \\
\hline verage & 0.11 & 0.07 & 0.15 & 0.18 & 0.14 & 0.10 & 0.16 & 0.20 & 0.17 & 0.15 & 0.19 & 0.22 & 0.16 & 0.17 & 0.18 & 0.19 \\
\hline
\end{tabular}


Table S13. Unscaled and scaled chemical shifts and its errors computed for isomers 1-8 at the $\mathrm{PCM} / \mathrm{mPW} 1 \mathrm{PW} 91 / 6-31+\mathrm{G}^{* *} / / \mathrm{B} 3 \mathrm{LYP} / 6-31 \mathrm{G}^{*}$ level of theory for compound $\mathbf{5}$

\begin{tabular}{|c|c|c|c|c|c|c|c|c|}
\hline \multicolumn{9}{|c|}{ Unscaled chemical shifts constants } \\
\hline Nuclei & Isomer 1 & Isomer 2 & Isomer 3 & Isomer 4 & Isomer 5 & Isomer 6 & Isomer 7 & Isomer 8 \\
\hline C 1 & 31.55 & 30.20 & 31.94 & 30.42 & 31.59 & 30.39 & 31.70 & 30.64 \\
\hline C 2 & 33.71 & 35.58 & 33.68 & 35.28 & 33.97 & 35.10 & 33.85 & 35.62 \\
\hline C 3 & 113.69 & 115.43 & 113.14 & 116.38 & 112.76 & 116.79 & 113.28 & 115.94 \\
\hline C 4 & 145.24 & 145.74 & 145.57 & 144.18 & 145.27 & 144.62 & 145.40 & 146.44 \\
\hline C 5 & 43.89 & 42.24 & 44.00 & 41.79 & 44.08 & 41.78 & 44.35 & 42.37 \\
\hline C 6 & 154.36 & 153.52 & 154.66 & 154.35 & 153.80 & 155.03 & 153.87 & 154.11 \\
\hline$C 7$ & 31.64 & 31.46 & 31.46 & 32.10 & 32.07 & 31.53 & 31.57 & 31.31 \\
\hline C 8 & 34.81 & 31.74 & 34.43 & 32.21 & 34.51 & 32.40 & 34.11 & 31.27 \\
\hline$C 9$ & 45.00 & 45.37 & 44.61 & 44.36 & 45.06 & 44.44 & 45.44 & 45.17 \\
\hline C 10 & 29.43 & 30.66 & 29.88 & 30.82 & 28.57 & 29.69 & 29.51 & 30.67 \\
\hline C 11 & 87.28 & 87.36 & 87.52 & 88.07 & 87.32 & 87.40 & 87.47 & 87.71 \\
\hline C 12 & 29.66 & 29.28 & 29.53 & 28.49 & 29.03 & 27.91 & 29.84 & 27.09 \\
\hline C 13 & 39.42 & 39.69 & 39.43 & 39.27 & 40.15 & 40.25 & 38.98 & 39.28 \\
\hline C 14 & 29.56 & 29.46 & 29.66 & 29.19 & 29.46 & 29.64 & 29.60 & 29.21 \\
\hline C 15 & 86.61 & 86.69 & 86.82 & 86.11 & 87.38 & 87.67 & 86.65 & 85.29 \\
\hline C 16 & 72.77 & 73.04 & 71.95 & 73.20 & 71.69 & 72.71 & 72.50 & 73.62 \\
\hline C 17 & 25.15 & 25.05 & 24.73 & 24.28 & 29.09 & 28.22 & 28.90 & 28.32 \\
\hline C 18 & 28.98 & 28.77 & 28.86 & 27.73 & 24.80 & 24.39 & 25.02 & 24.96 \\
\hline C 19 & 19.64 & 19.74 & 19.49 & 20.12 & 19.45 & 19.94 & 19.94 & 19.92 \\
\hline C 20 & 100.88 & 100.03 & 99.93 & 99.90 & 100.31 & 99.63 & 100.58 & 99.79 \\
\hline H 1 & 2.04 & 1.90 & 2.03 & 1.88 & 2.00 & 1.86 & 2.04 & 1.86 \\
\hline $\mathrm{H} 2 \mathrm{a}$ & 2.35 & 2.09 & 2.37 & 2.08 & 2.30 & 2.04 & 2.33 & 2.01 \\
\hline $\mathrm{H} 2 \mathrm{~b}$ & 1.88 & 1.79 & 1.89 & 1.70 & 1.87 & 1.71 & 1.86 & 1.70 \\
\hline H 3 & 5.83 & 5.87 & 5.82 & 5.86 & 5.86 & 5.75 & 5.80 & 5.78 \\
\hline H 5 & 2.02 & 2.10 & 2.00 & 1.95 & 1.98 & 2.08 & 1.99 & 2.01 \\
\hline $\mathrm{H} 7 \mathrm{a}$ & 2.28 & 2.27 & 2.26 & 2.28 & 2.19 & 2.23 & 2.24 & 2.23 \\
\hline $\mathrm{H} 7 \mathrm{~b}$ & 2.22 & 2.23 & 2.23 & 2.25 & 2.12 & 2.18 & 2.20 & 2.23 \\
\hline H 8a & 1.96 & 2.25 & 2.02 & 2.22 & 1.89 & 2.12 & 2.00 & 2.25 \\
\hline $\mathrm{H} 8 \mathrm{~b}$ & 1.84 & 1.56 & 1.92 & 1.66 & 1.83 & 1.55 & 1.94 & 1.61 \\
\hline H 10 & 1.77 & 1.63 & 1.79 & 1.56 & 1.56 & 1.80 & 1.72 & 1.95 \\
\hline H 12a-c & 1.31 & 1.34 & 1.28 & 1.32 & 1.30 & 1.34 & 1.34 & 1.27 \\
\hline H 13a & 2.28 & 2.25 & 2.10 & 2.17 & 2.02 & 2.25 & 2.36 & 1.94 \\
\hline $\mathrm{H} 13 \mathrm{~b}$ & 1.85 & 1.84 & 1.93 & 1.65 & 1.97 & 1.79 & 1.74 & 1.82 \\
\hline H 14a & 1.72 & 1.76 & 1.69 & 1.80 & 2.10 & 2.03 & 2.03 & 2.05 \\
\hline $\mathrm{H} 14 \mathrm{~b}$ & 1.98 & 1.99 & 2.08 & 1.94 & 1.69 & 1.76 & 1.66 & 1.98 \\
\hline H 15 & 4.03 & 3.99 & 3.87 & 3.88 & 3.95 & 4.03 & 4.07 & 4.07 \\
\hline H 17a-c & 1.01 & 1.01 & 1.02 & 1.02 & 1.18 & 1.15 & 1.18 & 1.15 \\
\hline H 18a-c & 1.17 & 1.17 & 1.16 & 1.12 & 1.02 & 1.06 & 0.97 & 0.99 \\
\hline H 19a-c & 0.96 & 1.04 & 0.99 & 0.99 & 0.98 & 0.97 & 0.98 & 1.00 \\
\hline H 20a & 5.22 & 5.17 & 5.15 & 5.14 & 5.14 & 5.13 & 5.16 & 5.20 \\
\hline $\mathrm{H} 20 \mathrm{~b}$ & 5.02 & 4.98 & 4.95 & 4.93 & 5.06 & 5.01 & 5.01 & 4.97 \\
\hline
\end{tabular}




\begin{tabular}{|c|c|c|c|c|c|c|c|c|}
\hline \multicolumn{9}{|c|}{ Scaled chemical shifts constants } \\
\hline Nuclei & Isomer 1 & Isomer 2 & Isomer 3 & Isomer 4 & Isomer 5 & Isomer 6 & Isomer 7 & Isomer 8 \\
\hline C 1 & 28.49 & 27.33 & 28.99 & 27.75 & 28.59 & 27.79 & 28.61 & 28.06 \\
\hline $\mathrm{C} 2$ & 30.70 & 32.82 & 30.78 & 32.71 & 31.04 & 32.57 & 30.81 & 33.12 \\
\hline $\mathrm{C} 3$ & 112.72 & 114.41 & 112.25 & 115.40 & 112.08 & 115.50 & 112.49 & 114.72 \\
\hline $\mathrm{C} 4$ & 145.07 & 145.38 & 145.51 & 143.75 & 145.52 & 143.75 & 145.50 & 145.70 \\
\hline C 5 & 41.15 & 39.62 & 41.36 & 39.35 & 41.43 & 39.35 & 41.61 & 39.98 \\
\hline C 6 & 154.42 & 153.34 & 154.83 & 154.12 & 154.30 & 154.32 & 154.22 & 153.49 \\
\hline C 7 & 28.58 & 28.61 & 28.50 & 29.46 & 29.09 & 28.94 & 28.47 & 28.74 \\
\hline C 8 & 31.83 & 28.90 & 31.55 & 29.58 & 31.60 & 29.82 & 31.08 & 28.70 \\
\hline C 9 & 42.28 & 42.82 & 41.98 & 41.97 & 42.45 & 42.05 & 42.73 & 42.82 \\
\hline C 10 & 26.31 & 27.79 & 26.88 & 28.17 & 25.49 & 27.07 & 26.35 & 28.09 \\
\hline C 11 & 85.64 & 85.73 & 85.98 & 86.54 & 85.92 & 85.66 & 85.95 & 86.03 \\
\hline C 12 & 26.56 & 26.38 & 26.52 & 25.78 & 25.96 & 25.27 & 26.69 & 24.45 \\
\hline C 13 & 36.56 & 37.02 & 36.67 & 36.78 & 37.40 & 37.80 & 36.09 & 36.84 \\
\hline C 14 & 26.45 & 26.57 & 26.66 & 26.50 & 26.40 & 27.02 & 26.45 & 26.61 \\
\hline C 15 & 84.94 & 85.05 & 85.26 & 84.54 & 85.97 & 85.93 & 85.10 & 83.58 \\
\hline C 16 & 70.76 & 71.10 & 70.02 & 71.38 & 69.83 & 70.75 & 70.55 & 71.72 \\
\hline C 17 & 21.93 & 22.06 & 21.60 & 21.49 & 26.02 & 25.58 & 25.73 & 25.70 \\
\hline C 18 & 25.86 & 25.86 & 25.84 & 25.01 & 21.61 & 21.69 & 21.74 & 22.28 \\
\hline C 19 & 16.28 & 16.64 & 16.23 & 17.25 & 16.11 & 17.18 & 16.52 & 17.17 \\
\hline C 20 & 99.58 & 98.67 & 98.70 & 98.59 & 99.28 & 98.08 & 99.42 & 98.30 \\
\hline H 1 & 1.92 & 1.82 & 1.91 & 1.83 & 1.92 & 1.79 & 1.93 & 1.79 \\
\hline $\mathrm{H} 2 \mathrm{a}$ & 2.20 & 1.99 & 2.24 & 2.02 & 2.19 & 1.96 & 2.20 & 1.93 \\
\hline $\mathrm{H} 2 \mathrm{~b}$ & 1.77 & 1.72 & 1.79 & 1.67 & 1.80 & 1.65 & 1.76 & 1.63 \\
\hline $\mathrm{H} 3$ & 5.40 & 5.45 & 5.47 & 5.48 & 5.46 & 5.39 & 5.39 & 5.39 \\
\hline H 5 & 1.90 & 2.00 & 1.88 & 1.90 & 1.90 & 1.99 & 1.88 & 1.92 \\
\hline $\mathrm{H} 7 \mathrm{a}$ & 2.14 & 2.15 & 2.12 & 2.20 & 2.09 & 2.13 & 2.11 & 2.13 \\
\hline $\mathrm{H} 7 \mathrm{~b}$ & 2.09 & 2.12 & 2.10 & 2.17 & 2.03 & 2.09 & 2.08 & 2.12 \\
\hline $\mathrm{H} 8 \mathrm{a}$ & 1.85 & 2.14 & 1.91 & 2.15 & 1.81 & 2.03 & 1.89 & 2.14 \\
\hline $\mathrm{H} 8 \mathrm{~b}$ & 1.73 & 1.51 & 1.81 & 1.63 & 1.76 & 1.51 & 1.83 & 1.56 \\
\hline H 10 & 1.68 & 1.57 & 1.68 & 1.54 & 1.52 & 1.74 & 1.63 & 1.87 \\
\hline H 12a-c & 1.25 & 1.30 & 1.21 & 1.32 & 1.28 & 1.32 & 1.28 & 1.24 \\
\hline H 13a & 2.14 & 2.13 & 1.98 & 2.10 & 1.93 & 2.15 & 2.22 & 1.85 \\
\hline $\mathrm{H} 13 \mathrm{~b}$ & 1.74 & 1.77 & 1.82 & 1.62 & 1.89 & 1.73 & 1.65 & 1.75 \\
\hline H 14a & 1.63 & 1.69 & 1.60 & 1.76 & 2.01 & 1.95 & 1.91 & 1.96 \\
\hline $\mathrm{H} 14 \mathrm{~b}$ & 1.87 & 1.90 & 1.96 & 1.89 & 1.64 & 1.70 & 1.57 & 1.90 \\
\hline H 15 & 3.75 & 3.73 & 3.64 & 3.67 & 3.70 & 3.80 & 3.80 & 3.82 \\
\hline H 17a-c & 0.98 & 1.00 & 0.97 & 1.04 & 1.17 & 1.14 & 1.13 & 1.13 \\
\hline H 18a-c & 1.13 & 1.15 & 1.10 & 1.14 & 1.02 & 1.05 & 0.94 & 0.98 \\
\hline H 19a-c & 0.93 & 1.03 & 0.94 & 1.02 & 0.98 & 0.97 & 0.95 & 0.99 \\
\hline H 20a & 4.84 & 4.81 & 4.84 & 4.82 & 4.80 & 4.82 & 4.81 & 4.86 \\
\hline $\mathrm{H} 20 \mathrm{~b}$ & 4.66 & 4.64 & 4.65 & 4.62 & 4.72 & 4.70 & 4.67 & 4.65 \\
\hline
\end{tabular}


Absolute unscaled error

Absolute scaled error

\begin{tabular}{|c|c|c|c|c|c|c|c|c|c|c|c|c|c|c|c|c|}
\hline & Isomer 1 & Isomer 2 & Isomer 3 & Isomer 4 & Isomer 5 & Isomer 6 & Isomer 7 & Isomer 8 & Isomer 1 & Isomer 2 & Isomer 3 & Isomer 4 & Isomer 5 & Isomer 6 & Isomer 7 & Isomer 8 \\
\hline C 1 & 5.6 & 4.3 & 6.0 & 4.5 & 5.7 & 4.5 & 5.8 & 4.7 & 2.6 & 1.4 & 3.1 & 1.9 & $\begin{array}{l}2.7 \\
\end{array}$ & $\begin{array}{l}1.9 \\
\end{array}$ & $\begin{array}{l}2.7 \\
\end{array}$ & 2.2 \\
\hline C 2 & 2.0 & 3.9 & 2.0 & 3.6 & 2.3 & 3.4 & 2.1 & 3.9 & 1.0 & 1.1 & 0.9 & 1.0 & 0.7 & 0.9 & 0.9 & 1.4 \\
\hline C 3 & 0.9 & 0.8 & 1.5 & 1.8 & 1.8 & 2.2 & 1.3 & 1.3 & 1.9 & 0.2 & 2.3 & 0.8 & 2.5 & 0.9 & 2.1 & 0.1 \\
\hline C 4 & 2.3 & 2.8 & 2.7 & 1.3 & 2.4 & 1.7 & 2.5 & 3.5 & 2.2 & 2.5 & 2.6 & 0.8 & 2.6 & 0.9 & 2.6 & 2.8 \\
\hline C 5 & 6.5 & 4.8 & 6.6 & 4.4 & 6.7 & 4.4 & 7.0 & 5.0 & 3.7 & 2.2 & 4.0 & 1.9 & 4.0 & 2.0 & 4.2 & 2.6 \\
\hline C 6 & 0.8 & 0.1 & 1.1 & 0.8 & 0.2 & 1.4 & 0.3 & 0.5 & 0.8 & 0.3 & 1.2 & 0.5 & 0.7 & 0.7 & 0.6 & 0.1 \\
\hline C 7 & 2.8 & 2.7 & 2.7 & 3.3 & 3.3 & 2.7 & 2.8 & 2.5 & 0.2 & 0.2 & 0.3 & 0.7 & 0.3 & 0.1 & 0.3 & 0.1 \\
\hline C 8 & 5.7 & 2.6 & 5.3 & 3.1 & 5.4 & 3.3 & 5.0 & 2.2 & 2.7 & 0.2 & 2.4 & 0.5 & 2.5 & 0.7 & 2.0 & 0.4 \\
\hline C9 & 4.9 & 5.3 & 4.5 & 4.3 & 5.0 & 4.3 & 5.3 & 5.1 & 2.2 & 2.7 & 1.9 & 1.9 & 2.4 & 1.9 & 2.6 & 2.7 \\
\hline C 10 & 4.3 & 5.6 & 4.8 & 5.7 & 3.5 & 4.6 & 4.4 & 5.6 & 1.2 & 2.7 & 1.8 & 3.1 & 0.4 & 2.0 & 1.3 & 3.0 \\
\hline C 11 & 2.1 & 2.2 & 2.3 & 2.9 & 2.1 & 2.2 & 2.3 & 2.5 & 0.4 & 0.5 & 0.8 & 1.3 & 0.7 & 0.5 & 0.7 & 0.8 \\
\hline C 12 & 2.6 & 2.2 & 2.4 & 1.4 & 1.9 & 0.8 & 2.7 & 0.0 & 0.5 & 0.7 & 0.6 & 1.3 & 1.1 & 1.8 & 0.4 & 2.6 \\
\hline C 13 & 2.1 & 2.4 & 2.1 & 2.0 & 2.9 & 3.0 & 1.7 & 2.0 & 0.7 & 0.3 & 0.6 & 0.5 & 0.1 & 0.5 & 1.2 & 0.5 \\
\hline C 14 & 3.0 & 2.9 & 3.1 & 2.6 & 2.9 & 3.0 & 3.0 & 2.6 & 0.2 & 0.0 & 0.1 & 0.1 & 0.2 & 0.4 & 0.2 & 0.0 \\
\hline C 15 & 0.3 & 0.4 & 0.5 & 0.2 & 1.1 & 1.4 & 0.3 & 1.0 & 1.4 & 1.3 & 1.0 & 1.8 & 0.3 & 0.4 & 1.2 & 2.7 \\
\hline C 16 & 1.7 & 1.9 & 0.8 & 2.1 & 0.6 & 1.6 & 1.4 & 2.5 & 0.3 & 0.0 & 1.1 & 0.3 & 1.3 & 0.4 & 0.5 & 0.6 \\
\hline C 17 & 0.9 & 0.8 & 0.4 & 0.0 & 4.8 & 3.9 & 4.6 & 4.0 & 2.4 & 2.2 & 2.7 & 2.8 & 1.7 & 1.3 & 1.4 & 1.4 \\
\hline C 18 & 1.5 & 1.3 & 1.4 & 0.2 & 2.7 & 3.1 & 2.5 & 2.5 & 1.6 & 1.6 & 1.7 & 2.5 & 5.9 & 5.8 & 5.8 & 5.2 \\
\hline C 19 & 0.4 & 0.5 & 0.3 & 0.9 & 0.3 & 0.7 & 0.7 & 0.7 & 2.9 & 2.6 & 3.0 & 2.0 & 3.1 & 2.0 & 2.7 & 2.0 \\
\hline C 20 & 1.4 & 2.3 & 2.4 & 2.4 & 2.0 & 2.7 & 1.7 & 2.5 & 2.7 & 3.6 & 3.6 & 3.7 & 3.0 & 4.2 & 2.9 & 4.0 \\
\hline Average & 2.6 & 2.5 & 2.6 & 2.4 & 2.9 & 2.7 & 2.9 & 2.7 & 1.6 & 1.3 & 1.8 & 1.5 & 1.8 & 1.5 & 1.8 & 1.8 \\
\hline H 1 & 0.17 & 0.03 & 0.16 & 0.01 & 0.13 & 0.01 & 0.17 & 0.01 & 0.05 & 0.05 & 0.04 & 0.04 & 0.05 & 0.08 & 0.06 & 0.08 \\
\hline $\mathrm{H} 2 \mathrm{a}$ & 0.22 & 0.04 & 0.24 & 0.05 & 0.17 & 0.09 & 0.20 & 0.12 & 0.07 & 0.14 & 0.11 & 0.11 & 0.06 & 0.17 & 0.07 & 0.20 \\
\hline $\mathrm{H} 2 \mathrm{~b}$ & 0.40 & 0.31 & 0.41 & 0.22 & 0.39 & 0.23 & 0.38 & 0.22 & 0.29 & 0.24 & 0.31 & 0.19 & 0.32 & 0.17 & 0.28 & 0.15 \\
\hline $\mathrm{H} 3$ & 0.39 & 0.43 & 0.38 & 0.42 & 0.42 & 0.31 & 0.36 & 0.34 & 0.04 & 0.01 & 0.03 & 0.04 & 0.02 & 0.05 & 0.05 & 0.05 \\
\hline $\mathrm{H} 5$ & 0.22 & 0.30 & 0.20 & 0.15 & 0.18 & 0.28 & 0.19 & 0.21 & 0.10 & 0.20 & 0.08 & 0.10 & 0.10 & 0.19 & 0.08 & 0.12 \\
\hline $\mathrm{H} 7 \mathrm{a}$ & 0.07 & 0.06 & 0.05 & 0.07 & 0.02 & 0.02 & 0.03 & 0.02 & 0.07 & 0.06 & 0.09 & 0.01 & 0.12 & 0.08 & 0.10 & 0.08 \\
\hline $\mathrm{H} 7 \mathrm{~b}$ & 0.08 & 0.09 & 0.09 & 0.11 & 0.02 & 0.04 & 0.06 & 0.09 & 0.05 & 0.02 & 0.04 & 0.03 & 0.11 & 0.05 & 0.06 & 0.02 \\
\hline $\mathrm{H} 8 \mathrm{a}$ & 0.18 & 0.11 & 0.12 & 0.08 & 0.25 & 0.02 & 0.14 & 0.11 & 0.29 & 0.00 & 0.23 & 0.01 & 0.33 & 0.11 & 0.25 & 0.00 \\
\hline $\mathrm{H} 8 \mathrm{~b}$ & 0.18 & 0.10 & 0.26 & 0.00 & 0.17 & 0.11 & 0.28 & 0.05 & 0.07 & 0.15 & 0.15 & 0.03 & 0.10 & 0.15 & 0.17 & 0.10 \\
\hline H 10 & 0.42 & 0.28 & 0.44 & 0.21 & 0.21 & 0.45 & 0.37 & 0.60 & 0.33 & 0.22 & 0.33 & 0.19 & 0.17 & 0.39 & 0.28 & 0.52 \\
\hline H 12a-c & 0.03 & 0.00 & 0.06 & 0.02 & 0.04 & 0.00 & 0.00 & 0.07 & 0.09 & 0.04 & 0.13 & 0.02 & 0.06 & 0.02 & 0.06 & 0.10 \\
\hline H $13 a$ & 0.22 & 0.19 & 0.04 & 0.11 & 0.04 & 0.19 & 0.30 & 0.12 & 0.08 & 0.07 & 0.08 & 0.04 & 0.13 & 0.09 & 0.16 & 0.21 \\
\hline H 13b & 0.09 & 0.08 & 0.17 & 0.11 & 0.21 & 0.03 & 0.02 & 0.06 & 0.02 & 0.01 & 0.06 & 0.14 & 0.13 & 0.03 & 0.11 & 0.01 \\
\hline H 14a & 0.10 & 0.06 & 0.13 & 0.02 & 0.28 & 0.21 & 0.21 & 0.23 & 0.19 & 0.13 & 0.22 & 0.06 & 0.19 & 0.13 & 0.09 & 0.14 \\
\hline H 14b & 0.16 & 0.17 & 0.26 & 0.12 & 0.13 & 0.06 & 0.16 & 0.16 & 0.05 & 0.08 & 0.14 & 0.07 & 0.18 & 0.12 & 0.25 & 0.08 \\
\hline $\mathrm{H} 15$ & 0.25 & 0.21 & 0.09 & 0.10 & 0.17 & 0.25 & 0.29 & 0.29 & 0.03 & 0.05 & 0.14 & 0.11 & 0.08 & 0.02 & 0.02 & 0.04 \\
\hline H $17 a-c$ & 0.12 & 0.12 & 0.11 & 0.11 & 0.05 & 0.02 & 0.05 & 0.02 & 0.15 & 0.13 & 0.16 & 0.09 & 0.04 & 0.01 & 0.00 & 0.00 \\
\hline H 18a-c & 0.06 & 0.06 & 0.07 & 0.11 & 0.21 & 0.17 & 0.26 & 0.24 & 0.10 & 0.08 & 0.13 & 0.09 & 0.21 & 0.18 & 0.29 & 0.25 \\
\hline H 19a-c & 0.08 & 0.00 & 0.05 & 0.05 & 0.06 & 0.07 & 0.06 & 0.04 & 0.11 & 0.01 & 0.10 & 0.02 & 0.06 & 0.07 & 0.09 & 0.05 \\
\hline H 20a & 0.44 & 0.39 & 0.37 & 0.36 & 0.36 & 0.35 & 0.38 & 0.42 & 0.06 & 0.03 & 0.06 & 0.04 & 0.02 & 0.04 & 0.03 & 0.08 \\
\hline $\mathrm{H} 20 \mathrm{~b}$ & 0.39 & 0.35 & 0.32 & 0.30 & 0.43 & 0.38 & 0.38 & 0.34 & 0.03 & 0.01 & 0.02 & 0.01 & 0.09 & 0.07 & 0.04 & 0.02 \\
\hline Average & 0.20 & 0.16 & 0.19 & 0.13 & 0.19 & 0.16 & 0.20 & 0.18 & 0.11 & 0.08 & 0.13 & 0.07 & 0.12 & 0.11 & 0.12 & 0.11 \\
\hline
\end{tabular}


Table S14. Unscaled and scaled chemical shifts and its errors computed for isomers 1-8 at the $\mathrm{PCM} / \mathrm{mPW} 1 \mathrm{PW} 91 / 6-31+\mathrm{G}^{* * / / B 3 L Y P} / 6-31 \mathrm{G}^{*}$ level of theory for compound isoelisabethatriene (vacuum).

\begin{tabular}{|c|c|c|c|c|}
\hline \multicolumn{5}{|c|}{ Unscaled chemical shifts constants } \\
\hline Nuclei & Isomer 1 & Isomer 2 & Isomer 3 & Isomer 4 \\
\hline C 1 & 132.55 & 132.20 & 132.05 & 132.15 \\
\hline $\mathrm{C} 2$ & 121.54 & 121.81 & 121.61 & 122.41 \\
\hline C 3 & 133.39 & 132.35 & 133.03 & 132.56 \\
\hline C 4 & 31.68 & 31.65 & 31.73 & 31.73 \\
\hline C 5 & 32.58 & 30.82 & 32.48 & 31.44 \\
\hline C 6 & 135.26 & 134.41 & 134.87 & 134.78 \\
\hline C 7 & 37.69 & 37.80 & 37.69 & 37.70 \\
\hline C 8 & 31.57 & 34.40 & 31.43 & 33.81 \\
\hline C 9 & 21.06 & 23.86 & 20.31 & 24.04 \\
\hline C 10 & 47.03 & 44.27 & 44.29 & 46.75 \\
\hline C 11 & 36.92 & 37.14 & 36.40 & 38.33 \\
\hline C 12 & 32.65 & 38.27 & 38.03 & 33.58 \\
\hline C 13 & 30.99 & 30.62 & 30.63 & 31.28 \\
\hline C 14 & 125.51 & 125.44 & 125.66 & 125.91 \\
\hline C 15 & 131.33 & 131.78 & 131.30 & 130.96 \\
\hline C 16 & 19.06 & 19.00 & 19.06 & 19.03 \\
\hline C 17 & 27.93 & 27.97 & 28.04 & 28.00 \\
\hline C 18 & 19.65 & 15.50 & 15.31 & 19.66 \\
\hline C 19 & 22.28 & 20.73 & 22.48 & 20.72 \\
\hline C 20 & 25.73 & 25.35 & 25.58 & 25.18 \\
\hline H 2 & 6.03 & 6.06 & 5.98 & 6.02 \\
\hline $\mathrm{H} 4 \mathrm{a}$ & 1.87 & 2.15 & 1.80 & 2.13 \\
\hline $\mathrm{H} 4 \mathrm{~b}$ & 2.15 & 1.84 & 2.19 & 1.82 \\
\hline H $5 a$ & 2.11 & 2.02 & 2.22 & 1.96 \\
\hline $\mathrm{H} 5 \mathrm{~b}$ & 2.02 & 2.23 & 1.94 & 2.25 \\
\hline H 7 & 1.88 & 2.30 & 1.85 & 2.27 \\
\hline H $8 \mathrm{a}$ & 1.36 & 1.15 & 1.40 & 1.13 \\
\hline H 8b & 1.62 & 1.65 & 1.62 & 1.66 \\
\hline $\mathrm{H} 9 \mathrm{a}$ & 1.59 & 1.52 & 1.64 & 1.61 \\
\hline H 9b & 1.42 & 1.42 & 1.31 & 1.41 \\
\hline H 10 & 2.07 & 2.21 & 2.23 & 2.06 \\
\hline H 11 & 1.83 & 1.90 & 1.89 & 1.80 \\
\hline $\mathrm{H} 12 \mathrm{a}$ & 1.36 & 1.25 & 1.24 & 0.90 \\
\hline $\mathrm{H} 12 \mathrm{~b}$ & 0.97 & 1.25 & 1.28 & 1.32 \\
\hline H 13a & 2.08 & 2.08 & 2.06 & 1.80 \\
\hline $\mathrm{H} 13 \mathrm{~b}$ & 1.84 & 2.04 & 2.07 & 2.07 \\
\hline H 14 & 5.42 & 5.51 & 5.48 & 5.41 \\
\hline H 19a-c & 1.03 & 0.86 & 1.05 & 0.87 \\
\hline H 18a-c & 0.93 & 0.65 & 0.66 & 0.93 \\
\hline H 16a-c & 1.59 & 1.61 & 1.64 & 1.60 \\
\hline H 17a-c & 1.67 & 1.71 & 1.69 & 1.65 \\
\hline H 20a-c & 1.83 & 1.79 & 1.80 & 1.82 \\
\hline
\end{tabular}




\begin{tabular}{|c|c|c|c|c|}
\hline \multicolumn{5}{|c|}{ Scaled chemical shifts constants } \\
\hline Nuclei & Isomer 1 & Isomer 2 & Isomer 3 & Isomer 4 \\
\hline C 1 & 131.93 & 131.74 & 131.51 & 131.66 \\
\hline $\mathrm{C} 2$ & 120.72 & 121.15 & 120.90 & 121.70 \\
\hline C 3 & 132.79 & 131.89 & 132.50 & 132.07 \\
\hline C 4 & 29.23 & 29.21 & 29.56 & 28.97 \\
\hline C 5 & 30.15 & 28.36 & 30.32 & 28.68 \\
\hline C 6 & 134.69 & 133.99 & 134.37 & 134.34 \\
\hline$C 7$ & 35.35 & 35.48 & 35.62 & 35.08 \\
\hline C 8 & 29.12 & 32.01 & 29.25 & 31.10 \\
\hline C 9 & 18.42 & 21.26 & 17.95 & 21.11 \\
\hline C 10 & 44.86 & 42.08 & 42.32 & 44.33 \\
\hline C 11 & 34.56 & 34.80 & 34.30 & 35.72 \\
\hline C 12 & 30.22 & 35.96 & 35.96 & 30.86 \\
\hline C 13 & 28.53 & 28.16 & 28.44 & 28.51 \\
\hline C 14 & 124.76 & 124.85 & 125.01 & 125.27 \\
\hline C 15 & 130.69 & 131.31 & 130.75 & 130.44 \\
\hline C 16 & 16.38 & 16.31 & 16.68 & 15.99 \\
\hline C 17 & 25.41 & 25.45 & 25.81 & 25.16 \\
\hline C 18 & 16.98 & 12.74 & 12.87 & 16.63 \\
\hline C 19 & 19.66 & 18.07 & 20.16 & 17.71 \\
\hline C 20 & 23.17 & 22.78 & 23.31 & 22.27 \\
\hline $\mathrm{H} 2$ & 5.91 & 5.80 & 5.82 & 5.88 \\
\hline $\mathrm{H} 4 \mathrm{a}$ & 1.91 & 2.16 & 1.83 & 2.17 \\
\hline $\mathrm{H} 4 \mathrm{~b}$ & 2.18 & 1.87 & 2.20 & 1.88 \\
\hline H $5 a$ & 2.15 & 2.04 & 2.23 & 2.01 \\
\hline $\mathrm{H} 5 \mathrm{~b}$ & 2.06 & 2.23 & 1.97 & 2.29 \\
\hline H 7 & 1.92 & 2.30 & 1.88 & 2.30 \\
\hline H $8 \mathrm{a}$ & 1.42 & 1.23 & 1.45 & 1.22 \\
\hline H $8 b$ & 1.67 & 1.69 & 1.66 & 1.72 \\
\hline H 9a & 1.65 & 1.57 & 1.68 & 1.68 \\
\hline $\mathrm{H} 9 \mathrm{~b}$ & 1.48 & 1.48 & 1.37 & 1.48 \\
\hline H 10 & 2.11 & 2.21 & 2.24 & 2.10 \\
\hline H 11 & 1.88 & 1.92 & 1.92 & 1.86 \\
\hline $\mathrm{H} 12 \mathrm{a}$ & 1.42 & 1.32 & 1.30 & 1.00 \\
\hline $\mathrm{H} 12 \mathrm{~b}$ & 1.05 & 1.32 & 1.34 & 1.40 \\
\hline H 13a & 2.12 & 2.09 & 2.08 & 1.86 \\
\hline $\mathrm{H} 13 \mathrm{~b}$ & 1.89 & 2.06 & 2.09 & 2.11 \\
\hline H 14 & 5.33 & 5.29 & 5.34 & 5.30 \\
\hline H 19a-c & 1.11 & 0.96 & 1.12 & 0.97 \\
\hline H 18a-c & 1.01 & 0.76 & 0.75 & 1.03 \\
\hline H 16a-c & 1.65 & 1.65 & 1.68 & 1.67 \\
\hline H 17a-c & 1.72 & 1.75 & 1.73 & 1.71 \\
\hline H 20a-c & 1.88 & 1.82 & 1.83 & 1.88 \\
\hline
\end{tabular}


Absolute unscaled error

Absolute scaled error

\begin{tabular}{|llll|llll}
\hline Isomer 1 & Isomer 2 & Isomer 3 & Isomer 4 & Isomer 1 & Isomer 2 & Isomer 3 & Isomer 4 \\
\hline
\end{tabular}

\begin{tabular}{|c|c|c|c|c|c|c|c|c|}
\hline C 1 & 2.2 & 1.9 & 1.7 & $\begin{array}{l}1.8 \\
\end{array}$ & 1.6 & 1.4 & 1.2 & 1.4 \\
\hline C 2 & 1.3 & 1.0 & 1.2 & 0.4 & 2.1 & 1.7 & 1.9 & 1.1 \\
\hline C 3 & 1.1 & 0.0 & 0.7 & 0.3 & 0.5 & 0.4 & 0.2 & 0.2 \\
\hline C 4 & 2.6 & 2.5 & 2.6 & 2.6 & 0.1 & 0.1 & 0.5 & 0.1 \\
\hline C 5 & 5.2 & 3.4 & 5.1 & 4.0 & 2.7 & 1.0 & 2.9 & 1.3 \\
\hline C 6 & 2.0 & 1.1 & 1.6 & 1.5 & 1.4 & 0.7 & 1.1 & 1.0 \\
\hline C 7 & 3.7 & 3.8 & 3.7 & 3.7 & 1.3 & 1.5 & 1.6 & 1.1 \\
\hline C 8 & 0.1 & 2.9 & 0.1 & 2.3 & 2.4 & 0.5 & 2.2 & 0.4 \\
\hline C 9 & 0.0 & 2.8 & 0.8 & 2.9 & 2.7 & 0.2 & 3.1 & 0.0 \\
\hline C 10 & 6.0 & 3.3 & 3.3 & 5.7 & 3.9 & 1.1 & 1.3 & 3.3 \\
\hline C 11 & 2.4 & 2.6 & 1.9 & 3.8 & 0.1 & 0.3 & 0.2 & 1.2 \\
\hline C 12 & 3.1 & 2.6 & 2.3 & 2.1 & 5.5 & 0.3 & 0.3 & 4.8 \\
\hline C 13 & 4.1 & 3.7 & 3.7 & 4.4 & 1.6 & 1.3 & 1.5 & 1.6 \\
\hline C 14 & 0.1 & 0.2 & 0.1 & 0.3 & 0.8 & 0.8 & 0.6 & 0.3 \\
\hline C 15 & 0.4 & 0.9 & 0.4 & 0.1 & 0.2 & 0.4 & 0.2 & 0.5 \\
\hline C 16 & 6.8 & 6.9 & 6.8 & 6.9 & 9.5 & 9.6 & 9.2 & 9.9 \\
\hline C 17 & 10.1 & 10.2 & 10.2 & 10.2 & 7.6 & 7.7 & 8.0 & 7.4 \\
\hline C 18 & 4.6 & 0.5 & 0.3 & 4.7 & 2.0 & 2.3 & 2.1 & 1.6 \\
\hline C 19 & 3.1 & 1.5 & 3.3 & 1.5 & 0.5 & 1.1 & 1.0 & 1.5 \\
\hline C 20 & 2.4 & 2.0 & 2.3 & 1.9 & 0.1 & 0.5 & 0.0 & 1.0 \\
\hline verage & 3.1 & 2.7 & 2.6 & 3.1 & 2.3 & 1.6 & 2.0 & 2.0 \\
\hline $\mathrm{H} 2$ & 0.25 & 0.28 & 0.20 & 0.24 & 0.13 & 0.02 & 0.04 & 0.10 \\
\hline $\mathrm{H} 4 \mathrm{a}$ & 0.21 & 0.07 & 0.28 & 0.05 & 0.17 & 0.08 & 0.25 & 0.09 \\
\hline $\mathrm{H} 4 \mathrm{~b}$ & 0.29 & 0.02 & 0.33 & 0.04 & 0.32 & 0.01 & 0.34 & 0.02 \\
\hline H 5a & 0.08 & 0.17 & 0.03 & 0.23 & 0.04 & 0.15 & 0.04 & 0.18 \\
\hline H 5b & 0.07 & 0.28 & 0.01 & 0.30 & 0.11 & 0.28 & 0.02 & 0.34 \\
\hline H 7 & 0.30 & 0.12 & 0.33 & 0.09 & 0.26 & 0.12 & 0.30 & 0.12 \\
\hline H 8a & 0.45 & 0.66 & 0.41 & 0.68 & 0.39 & 0.58 & 0.36 & 0.59 \\
\hline $\mathrm{H} 8 \mathrm{~b}$ & 0.41 & 0.44 & 0.41 & 0.45 & 0.46 & 0.48 & 0.45 & 0.51 \\
\hline H 9a & 0.06 & 0.13 & 0.01 & 0.04 & 0.00 & 0.08 & 0.03 & 0.03 \\
\hline $\mathrm{H} 9 \mathrm{~b}$ & 0.00 & 0.00 & 0.11 & 0.01 & 0.06 & 0.06 & 0.05 & 0.06 \\
\hline H 10 & 0.17 & 0.03 & 0.01 & 0.18 & 0.13 & 0.03 & 0.00 & 0.14 \\
\hline H 11 & 0.17 & 0.10 & 0.11 & 0.20 & 0.12 & 0.08 & 0.08 & 0.14 \\
\hline H 12a & 0.09 & 0.20 & 0.21 & 0.55 & 0.03 & 0.13 & 0.15 & 0.45 \\
\hline $\mathrm{H} 12 \mathrm{~b}$ & 0.40 & 0.12 & 0.09 & 0.05 & 0.32 & 0.05 & 0.03 & 0.03 \\
\hline H 13a & 0.03 & 0.03 & 0.05 & 0.31 & 0.01 & 0.02 & 0.03 & 0.25 \\
\hline $\mathrm{H} 13 \mathrm{~b}$ & 0.23 & 0.03 & 0.00 & 0.00 & 0.18 & 0.01 & 0.02 & 0.04 \\
\hline H 14 & 0.15 & 0.24 & 0.21 & 0.14 & 0.06 & 0.02 & 0.07 & 0.03 \\
\hline 19a-c & 0.06 & 0.11 & 0.08 & 0.10 & 0.14 & 0.01 & 0.15 & 0.00 \\
\hline 1 18a-c & 0.09 & 0.19 & 0.18 & 0.09 & 0.17 & 0.08 & 0.09 & 0.19 \\
\hline $16 a-c$ & 0.12 & 0.10 & 0.07 & 0.11 & 0.06 & 0.06 & 0.03 & 0.04 \\
\hline $17 a-c$ & 0.08 & 0.12 & 0.10 & 0.06 & 0.13 & 0.16 & 0.14 & 0.12 \\
\hline $20 a-c$ & 0.07 & 0.03 & 0.04 & 0.06 & 0.12 & 0.06 & 0.07 & 0.12 \\
\hline verage & 0.17 & 0.16 & 0.15 & 0.18 & 0.16 & 0.12 & 0.13 & 0.16 \\
\hline
\end{tabular}


Table S15. Unscaled and scaled chemical shifts and its errors computed for isomers 1-8 at the $\mathrm{PCM} / \mathrm{mPW} 1 \mathrm{PW} 91 / 6-31+\mathrm{G}^{* *} / / \mathrm{B} 3 \mathrm{LYP} / 6-31 \mathrm{G}^{*}$ level of theory for compound isoelisabethatriene benzene

\begin{tabular}{|c|c|c|c|c|}
\hline \multicolumn{5}{|c|}{ Unscaled chemical shifts constants } \\
\hline Nuclei & Isomer 1 & Isomer 2 & Isomer 3 & Isomer 4 \\
\hline C 1 & 132.43 & 132.01 & 131.90 & 131.98 \\
\hline $\mathrm{C} 2$ & 121.12 & 121.38 & 121.20 & 121.99 \\
\hline C 3 & 134.05 & 133.06 & 133.73 & 133.22 \\
\hline C 4 & 31.56 & 31.52 & 31.60 & 31.62 \\
\hline C 5 & 32.47 & 30.69 & 32.38 & 31.32 \\
\hline C 6 & 135.59 & 134.76 & 135.20 & 135.11 \\
\hline C 7 & 37.61 & 37.75 & 37.61 & 37.65 \\
\hline C 8 & 31.46 & 34.28 & 31.34 & 33.67 \\
\hline C9 & 21.00 & 23.77 & 20.21 & 23.94 \\
\hline C 10 & 46.96 & 44.23 & 44.25 & 46.66 \\
\hline C 11 & 36.99 & 37.16 & 36.42 & 38.38 \\
\hline C 12 & 32.69 & 38.21 & 37.97 & 33.61 \\
\hline C 13 & 30.89 & 30.49 & 30.51 & 31.18 \\
\hline C 14 & 125.22 & 125.18 & 125.41 & 125.63 \\
\hline C 15 & 131.84 & 132.26 & 131.77 & 131.48 \\
\hline C 16 & 18.90 & 18.83 & 18.88 & 18.86 \\
\hline C 17 & 27.71 & 27.74 & 27.83 & 27.78 \\
\hline C 18 & 19.47 & 15.33 & 15.15 & 19.46 \\
\hline C 19 & 22.10 & 20.56 & 22.29 & 20.57 \\
\hline C 20 & 25.51 & 25.12 & 25.37 & 24.95 \\
\hline $\mathrm{H} 2$ & 6.05 & 6.08 & 6.01 & 6.05 \\
\hline $\mathrm{H} 4 \mathrm{a}$ & 1.91 & 2.16 & 1.85 & 2.13 \\
\hline $\mathrm{H} 4 \mathrm{~b}$ & 2.16 & 1.88 & 2.19 & 1.86 \\
\hline H 5a & 2.11 & 2.07 & 2.21 & 2.00 \\
\hline $\mathrm{H} 5 \mathrm{~b}$ & 2.06 & 2.23 & 1.99 & 2.24 \\
\hline H 7 & 1.91 & 2.31 & 1.88 & 2.29 \\
\hline $\mathrm{H} 8 \mathrm{a}$ & 1.38 & 1.15 & 1.41 & 1.13 \\
\hline $\mathrm{H} 8 \mathrm{~b}$ & 1.61 & 1.67 & 1.61 & 1.67 \\
\hline H 9a & 1.59 & 1.54 & 1.65 & 1.62 \\
\hline $\mathrm{H} 9 \mathrm{~b}$ & 1.43 & 1.42 & 1.32 & 1.41 \\
\hline H 10 & 2.09 & 2.23 & 2.25 & 2.08 \\
\hline H 11 & 1.84 & 1.91 & 1.90 & 1.81 \\
\hline $\mathrm{H} 12 \mathrm{a}$ & 1.32 & 1.26 & 1.24 & 0.91 \\
\hline $\mathrm{H} 12 \mathrm{~b}$ & 0.98 & 1.25 & 1.28 & 1.28 \\
\hline $\mathrm{H} 13 \mathrm{a}$ & 2.10 & 2.10 & 2.07 & 1.80 \\
\hline $\mathrm{H} 13 \mathrm{~b}$ & 1.84 & 2.05 & 2.08 & 2.09 \\
\hline H 14 & 5.45 & 5.54 & 5.50 & 5.43 \\
\hline H 19a-c & 1.62 & 1.63 & 1.67 & 1.62 \\
\hline H 18a-c & 1.69 & 1.73 & 1.70 & 1.67 \\
\hline H $16 a-c$ & 0.94 & 0.64 & 0.65 & 0.95 \\
\hline H 17a-c & 1.04 & 0.87 & 1.05 & 0.88 \\
\hline H 20a-c & 1.84 & 1.82 & 1.82 & 1.84 \\
\hline
\end{tabular}




\begin{tabular}{|c|c|c|c|c|}
\hline \multicolumn{5}{|c|}{ Scaled chemical shifts constants } \\
\hline Nuclei & Isomer 1 & Isomer 2 & Isomer 3 & Isomer 4 \\
\hline C 1 & 131.67 & 131.41 & 131.22 & 131.35 \\
\hline $\mathrm{C} 2$ & 120.18 & 120.60 & 120.37 & 121.16 \\
\hline C 3 & 133.31 & 132.48 & 133.07 & 132.61 \\
\hline C 4 & 29.22 & 29.20 & 29.55 & 28.98 \\
\hline C 5 & 30.14 & 28.36 & 30.34 & 28.67 \\
\hline C 6 & 134.88 & 134.21 & 134.56 & 134.54 \\
\hline C 7 & 35.36 & 35.54 & 35.64 & 35.13 \\
\hline C 8 & 29.12 & 32.01 & 29.28 & 31.07 \\
\hline C 9 & 18.49 & 21.32 & 18.00 & 21.15 \\
\hline C 10 & 44.86 & 42.13 & 42.37 & 44.32 \\
\hline C 11 & 34.73 & 34.94 & 34.43 & 35.88 \\
\hline C 12 & 30.37 & 36.01 & 36.00 & 31.01 \\
\hline C 13 & 28.54 & 28.15 & 28.44 & 28.53 \\
\hline C 14 & 124.35 & 124.46 & 124.64 & 124.87 \\
\hline C 15 & 131.07 & 131.66 & 131.08 & 130.84 \\
\hline C 16 & 16.36 & 16.29 & 16.65 & 15.97 \\
\hline C 17 & 25.31 & 25.36 & 25.73 & 25.06 \\
\hline C 18 & 16.94 & 12.73 & 12.87 & 16.58 \\
\hline C 19 & 19.61 & 18.05 & 20.11 & 17.71 \\
\hline C 20 & 23.07 & 22.69 & 23.23 & 22.18 \\
\hline $\mathrm{H} 2$ & 5.90 & 5.79 & 5.82 & 5.88 \\
\hline $\mathrm{H} 4 \mathrm{a}$ & 1.94 & 2.15 & 1.87 & 2.16 \\
\hline $\mathrm{H} 4 \mathrm{~b}$ & 2.18 & 1.89 & 2.19 & 1.90 \\
\hline H 5a & 2.13 & 2.07 & 2.21 & 2.04 \\
\hline $\mathrm{H} 5 \mathrm{~b}$ & 2.08 & 2.22 & 2.00 & 2.26 \\
\hline $\mathrm{H} 7$ & 1.94 & 2.29 & 1.90 & 2.31 \\
\hline $\mathrm{H} 8 \mathrm{a}$ & 1.43 & 1.21 & 1.45 & 1.21 \\
\hline $\mathrm{H} 8 \mathrm{~b}$ & 1.65 & 1.70 & 1.64 & 1.72 \\
\hline H 9a & 1.63 & 1.58 & 1.68 & 1.68 \\
\hline $\mathrm{H} 9 \mathrm{~b}$ & 1.48 & 1.46 & 1.37 & 1.48 \\
\hline H 10 & 2.11 & 2.22 & 2.25 & 2.11 \\
\hline H 11 & 1.87 & 1.92 & 1.92 & 1.86 \\
\hline $\mathrm{H} 12 \mathrm{a}$ & 1.38 & 1.32 & 1.29 & 1.00 \\
\hline $\mathrm{H} 12 \mathrm{~b}$ & 1.05 & 1.31 & 1.33 & 1.35 \\
\hline H 13a & 2.12 & 2.10 & 2.08 & 1.85 \\
\hline $\mathrm{H} 13 \mathrm{~b}$ & 1.87 & 2.05 & 2.09 & 2.12 \\
\hline H 14 & 5.33 & 5.29 & 5.33 & 5.29 \\
\hline H 19a-c & 1.66 & 1.66 & 1.70 & 1.68 \\
\hline H $18 a-c$ & 1.73 & 1.75 & 1.73 & 1.72 \\
\hline H 16a-c & 1.01 & 0.74 & 0.73 & 1.04 \\
\hline H $17 a-c$ & 1.11 & 0.95 & 1.11 & 0.97 \\
\hline H 20a-c & 1.87 & 1.84 & 1.84 & 1.88 \\
\hline
\end{tabular}


Absolute unscaled error

Absolute scaled error

\begin{tabular}{|llllllll}
\hline Isomer 1 & Isomer 2 & Isomer 3 & Isomer 4 & Isomer 1 & Isomer 2 & Isomer 3 & Isomer 4 \\
\hline
\end{tabular}

\begin{tabular}{|c|c|c|c|c|c|c|c|c|}
\hline & & & & & & & & \\
\hline C 1 & 2.1 & $\begin{array}{l}1.7 \\
\end{array}$ & $\begin{array}{l}1.6 \\
\end{array}$ & $\begin{array}{l}1.7 \\
\end{array}$ & \begin{tabular}{ll|}
1.4 \\
\end{tabular} & $\begin{array}{l}1.1 \\
\end{array}$ & 0.9 & $\begin{array}{ll}1.0 \\
\end{array}$ \\
\hline C 2 & 1.7 & 1.4 & 1.6 & 0.8 & 2.6 & 2.2 & 2.4 & 1.6 \\
\hline C 3 & 1.7 & 0.8 & 1.4 & 0.9 & 1.0 & 0.2 & 0.8 & 0.3 \\
\hline C 4 & 2.5 & 2.4 & 2.5 & 2.5 & 0.1 & 0.1 & 0.4 & 0.1 \\
\hline C 5 & 5.1 & 3.3 & 5.0 & 3.9 & 2.7 & 1.0 & 2.9 & 1.3 \\
\hline C 6 & 2.3 & 1.5 & 1.9 & 1.8 & 1.6 & 0.9 & 1.3 & 1.2 \\
\hline C 7 & 3.6 & 3.7 & 3.6 & 3.6 & 1.4 & 1.5 & 1.6 & 1.1 \\
\hline C 8 & 0.0 & 2.8 & 0.2 & 2.2 & 2.4 & 0.5 & 2.2 & 0.4 \\
\hline C 9 & 0.1 & 2.7 & 0.9 & 2.8 & 2.6 & 0.2 & 3.1 & 0.0 \\
\hline C 10 & 6.0 & 3.2 & 3.2 & 5.7 & 3.9 & 1.1 & 1.4 & 3.3 \\
\hline C 11 & 2.5 & 2.7 & 1.9 & 3.9 & 0.2 & 0.4 & 0.1 & 1.4 \\
\hline C 12 & 3.0 & 2.5 & 2.3 & 2.1 & 5.3 & 0.3 & 0.3 & 4.7 \\
\hline C 13 & 4.0 & 3.6 & 3.6 & 4.3 & 1.6 & 1.3 & 1.5 & 1.6 \\
\hline C 14 & 0.4 & 0.4 & 0.2 & 0.0 & 1.3 & 1.1 & 1.0 & 0.7 \\
\hline C 15 & 0.9 & 1.4 & 0.9 & 0.6 & 0.2 & 0.8 & 0.2 & 0.1 \\
\hline C 16 & 7.0 & 7.1 & 7.0 & 7.0 & 9.5 & 9.6 & 9.2 & 9.9 \\
\hline C 17 & 9.9 & 9.9 & 10.0 & 10.0 & 7.5 & 7.6 & 7.9 & 7.3 \\
\hline C 18 & 4.5 & 0.3 & 0.1 & 4.5 & 1.9 & 2.3 & 2.1 & 1.6 \\
\hline C 19 & 2.9 & 1.4 & 3.1 & 1.4 & 0.4 & 1.1 & 0.9 & 1.5 \\
\hline C 20 & 2.2 & 1.8 & 2.1 & 1.6 & 0.2 & 0.6 & 0.1 & 1.1 \\
\hline verage & 3.1 & 2.7 & 2.7 & 3.1 & 2.4 & 1.7 & 2.0 & 2.0 \\
\hline $\mathrm{H} 2$ & 0.27 & 0.30 & 0.23 & 0.27 & 0.12 & 0.01 & 0.04 & 0.10 \\
\hline H 4a & 0.17 & 0.08 & 0.23 & 0.05 & 0.14 & 0.07 & 0.21 & 0.08 \\
\hline $\mathrm{H} 4 \mathrm{~b}$ & 0.30 & 0.02 & 0.33 & 0.00 & 0.32 & 0.03 & 0.33 & 0.04 \\
\hline H 5a & 0.08 & 0.12 & 0.02 & 0.19 & 0.06 & 0.12 & 0.02 & 0.15 \\
\hline H 5b & 0.11 & 0.28 & 0.04 & 0.29 & 0.13 & 0.27 & 0.05 & 0.31 \\
\hline H7 & 0.27 & 0.13 & 0.30 & 0.11 & 0.24 & 0.11 & 0.28 & 0.13 \\
\hline H 8a & 0.43 & 0.66 & 0.40 & 0.68 & 0.38 & 0.60 & 0.36 & 0.60 \\
\hline H 8b & 0.40 & 0.46 & 0.40 & 0.46 & 0.44 & 0.49 & 0.43 & 0.51 \\
\hline H 9a & 0.06 & 0.11 & 0.00 & 0.03 & 0.02 & 0.07 & 0.03 & 0.03 \\
\hline H 9b & 0.01 & 0.00 & 0.10 & 0.01 & 0.06 & 0.04 & 0.05 & 0.06 \\
\hline H 10 & 0.15 & 0.01 & 0.01 & 0.16 & 0.13 & 0.02 & 0.01 & 0.13 \\
\hline H 11 & 0.16 & 0.09 & 0.10 & 0.19 & 0.13 & 0.08 & 0.08 & 0.14 \\
\hline H 12a & 0.13 & 0.19 & 0.21 & 0.54 & 0.07 & 0.13 & 0.16 & 0.45 \\
\hline $\mathrm{H} 12 \mathrm{~b}$ & 0.39 & 0.12 & 0.09 & 0.09 & 0.32 & 0.06 & 0.04 & 0.02 \\
\hline H 13a & 0.01 & 0.01 & 0.04 & 0.31 & 0.01 & 0.01 & 0.03 & 0.26 \\
\hline $\mathrm{H} 13 \mathrm{~b}$ & 0.23 & 0.02 & 0.01 & 0.02 & 0.20 & 0.02 & 0.02 & 0.05 \\
\hline H 14 & 0.18 & 0.27 & 0.23 & 0.16 & 0.06 & 0.02 & 0.06 & 0.02 \\
\hline 19a-c & 0.09 & 0.08 & 0.04 & 0.09 & 0.05 & 0.05 & 0.01 & 0.03 \\
\hline $18 a-c$ & 0.10 & 0.14 & 0.11 & 0.08 & 0.14 & 0.16 & 0.14 & 0.13 \\
\hline $16 a-c$ & 0.10 & 0.20 & 0.19 & 0.11 & 0.17 & 0.10 & 0.11 & 0.20 \\
\hline $17 a-c$ & 0.07 & 0.10 & 0.08 & 0.09 & 0.14 & 0.02 & 0.14 & 0.00 \\
\hline $20 \mathrm{a}-\mathrm{c}$ & 0.08 & 0.06 & 0.06 & 0.08 & 0.11 & 0.08 & 0.08 & 0.12 \\
\hline verage & 0.17 & 0.16 & 0.15 & 0.18 & 0.16 & 0.12 & 0.12 & 0.16 \\
\hline
\end{tabular}


1. DP4+ results of compounds 1-5 and isoelisabethatriene

Table S16. DP4+ results obtained using experimental data of compound 1 versus isomers 1-8

\begin{tabular}{|c|c|c|c|c|c|c|c|c|}
\hline \multirow{3}{*}{$\begin{array}{l}\text { Functional } \\
\text { mPW1PW91 }\end{array}$} & \multirow{2}{*}{\multicolumn{2}{|c|}{$\begin{array}{l}\text { Solvent? } \\
\text { PCM }\end{array}$}} & \multirow{2}{*}{\multicolumn{2}{|c|}{$\begin{array}{l}\text { Basis Set } \\
6-31+G(d, p)\end{array}$}} & \multirow{2}{*}{\multicolumn{2}{|c|}{$\begin{array}{l}\text { Type of Data } \\
\text { Shielding Tensors }\end{array}$}} & & \\
\hline & & & & & & & & \\
\hline & Isomer 1 & Isomer 2 & Isomer 3 & Isomer 4 & Isomer 5 & Isomer 6 & Isomer 7 & Isomer 8 \\
\hline sDP4+ (H data) & $0.00 \%$ & $0.00 \%$ & $0.00 \%$ & oOl $92.61 \%$ & $0.00 \%$ & $0.00 \%$ & $0.00 \%$ & $7.39 \%$ \\
\hline sDP4+ (C data) & $8.96 \%$ & $0.02 \%$ & $0.16 \%$ & $64.61 \%$ & $13.74 \%$ & $0.02 \%$ & $0.66 \%$ & $11.83 \%$ \\
\hline sDP4+ (all data) & $0.00 \%$ & $0.00 \%$ & $0.00 \%$ & ${ }_{0} 0 \| 98.56 \%$ & $0.00 \%$ & $0.00 \%$ & $0.00 \%$ & $1.44 \%$ \\
\hline uDP4+ (H data) & $0.00 \%$ & $0.00 \%$ & $0.00 \%$ & old $85.57 \%$ & $0.00 \%$ & $0.00 \%$ & $0.00 \%$ & $14.42 \%$ \\
\hline uDP4+ (C data) & $2.48 \%$ & $0.00 \%$ & $0.01 \%$ & $69.22 \%$ & $1.79 \%$ & $0.00 \%$ & $0.04 \%$ & $26.46 \%$ \\
\hline uDP4+ (all data) & $0.00 \%$ & $0.00 \%$ & $0.00 \%$ & old $93.95 \%$ & $0.00 \%$ & $0.00 \%$ & $0.00 \%$ & $6.05 \%$ \\
\hline DP4+ (H data) & $0.00 \%$ & $0.00 \%$ & $0.00 \%$ & old $988.67 \%$ & $0.00 \%$ & $0.00 \%$ & $0.00 \%$ & $1.33 \%$ \\
\hline DP4+ (C data) & $0.46 \%$ & $0.00 \%$ & $0.00 \%$ & old $92.55 \%$ & $0.51 \%$ & $0.00 \%$ & $0.00 \%$ & $6.48 \%$ \\
\hline DP4+ (all data) & $0.00 \%$ & $0.00 \%$ & $0.00 \%$ & | o01|99.91\% & $0.00 \%$ & $0.00 \%$ & $0.00 \%$ & $0.09 \%$ \\
\hline
\end{tabular}

Table S17. DP4+ results obtained using experimental data of compound 2 versus isomers 1-8

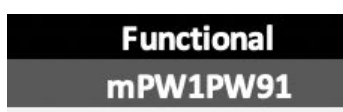

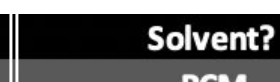

PCM

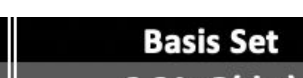

$6-31+G(d, p)$
Type of Data

Shielding Tensors

\begin{tabular}{|c|c|c|c|c|c|c|c|c|}
\hline & Isomer 1 & Isomer 2 & Isomer 3 & Isomer 4 & Isomer 5 & Isomer 6 & Isomer 7 & Isomer 8 \\
\hline sDP4+ (H data) & $15.43 \%$ & $22.95 \%$ & $14.31 \%$ & $18.51 \%$ & $0.89 \%$ & $2.52 \%$ & $25.04 \%$ & $0.36 \%$ \\
\hline sDP4+ (C data) & $67.33 \%$ & $0.04 \%$ & $32.43 \%$ & $0.10 \%$ & $0.09 \%$ & $0.00 \%$ & $0.01 \%$ & $0.00 \%$ \\
\hline sDP4+ (all data) & $68.98 \%$ & $0.05 \%$ & $30.82 \%$ & $0.12 \%$ & $0.01 \%$ & $0.00 \%$ & $0.02 \%$ & $0.00 \%$ \\
\hline uDP4+ (H data) & $7.46 \%$ & $39.12 \%$ & $9.63 \%$ & $13.95 \%$ & $0.47 \%$ & $2.72 \%$ & $26.03 \%$ & $0.61 \%$ \\
\hline uDP4+ (C data) & ol $78.04 \%$ & $0.64 \%$ & $19.37 \%$ & $1.88 \%$ & $0.07 \%$ & $0.00 \%$ & $0.01 \%$ & $0.00 \%$ \\
\hline uDP4+ (all data) & 70.99\% & $3.04 \%$ & $22.75 \%$ & $3.19 \%$ & $0.00 \%$ & $0.00 \%$ & $0.02 \%$ & $0.00 \%$ \\
\hline DP4+ (H data) & oed| $5.57 \%$ & $43.41 \%$ & $6.67 \%$ & $12.48 \%$ & $0.02 \%$ & $0.33 \%$ & $31.51 \%$ & $0.01 \%$ \\
\hline DP4+ (C data) & ${ }_{0} 0 \| 89.32 \%$ & $0.00 \%$ & $10.68 \%$ & $0.00 \%$ & $0.00 \%$ & $0.00 \%$ & $0.00 \%$ & $0.00 \%$ \\
\hline DP4+ (all data) & ${ }_{000} 087.47 \%$ & $0.00 \%$ & $12.52 \%$ & $0.01 \%$ & $0.00 \%$ & $0.00 \%$ & $0.00 \%$ & $0.00 \%$ \\
\hline
\end{tabular}


Table S18. DP4+ results obtained using experimental data of compound 3 versus isomers 1-16

\begin{tabular}{|c|c|c|c|c|c|c|c|c|c|c|c|c|c|c|c|c|}
\hline \multirow{3}{*}{$\begin{array}{l}\text { Functional } \\
\text { mPW1PW91 }\end{array}$} & \multirow{2}{*}{\multicolumn{2}{|c|}{$\begin{array}{l}\text { Solvent? } \\
\text { PCM }\end{array}$}} & \multicolumn{2}{|c|}{$\begin{array}{c}\text { Basis Set } \\
6-31+c(d, p)\end{array}$} & \multicolumn{2}{|c|}{ Type of Data } & & & & & & & & & & \\
\hline & & & 6-31+ & $s(d, p)$ & Shieldil & Tensors & & & & & & & & & & \\
\hline & Isomer 1 & Isomer 2 & Isomer 3 & Isomer 4 & Isomer 5 & Isomer 6 & Isomer 7 & Isomer 8 & Isomer 9 & Isomer 10 & Isomer 11 & Isomer 12 & Isomer 13 & Isomer 14 & Isomer 15 & Isomer 16 \\
\hline SDP4+ (H data) & $0.00 \%$ & $0.00 \%$ & $0.00 \%$ & $0.00 \%$ & $0.01 \%$ & $0.01 \%$ & $0.00 \%$ & $0.01 \%$ & ool $\mid 99.52 \%$ & $0.00 \%$ & $0.45 \%$ & $0.00 \%$ & $0.00 \%$ & $0.00 \%$ & $0.00 \%$ & $0.00 \%$ \\
\hline SDP4+ (C data) & $0.15 \%$ & $0.00 \%$ & $0.00 \%$ & $0.00 \%$ & $0.00 \%$ & $0.00 \%$ & $0.00 \%$ & $0.00 \%$ & oll $999.82 \%$ & $0.00 \%$ & $0.03 \%$ & $0.00 \%$ & $0.00 \%$ & $0.00 \%$ & $0.00 \%$ & $0.00 \%$ \\
\hline SDP4+ (all data) & $0.00 \%$ & $0.00 \%$ & $0.00 \%$ & $0.00 \%$ & $0.00 \%$ & $0.00 \%$ & $0.00 \%$ & $0.00 \%$ & onf $100.00 \%$ & $0.00 \%$ & $0.00 \%$ & $0.00 \%$ & $0.00 \%$ & $0.00 \%$ & $0.00 \%$ & $0.00 \%$ \\
\hline uDP4+ (H data) & $0.01 \%$ & $0.00 \%$ & $0.00 \%$ & $0.00 \%$ & $0.00 \%$ & $0.00 \%$ & $0.00 \%$ & $0.00 \%$ & ooll|99.96\% & $0.00 \%$ & $0.02 \%$ & $0.00 \%$ & $0.00 \%$ & $0.00 \%$ & $0.00 \%$ & $0.00 \%$ \\
\hline uDP4+ (C data) & $0.16 \%$ & $0.00 \%$ & $0.00 \%$ & $0.00 \%$ & $0.00 \%$ & $0.00 \%$ & $0.00 \%$ & $0.00 \%$ & ofll $999.79 \%$ & $0.00 \%$ & $0.05 \%$ & $0.00 \%$ & $0.00 \%$ & $0.00 \%$ & $0.00 \%$ & $0.00 \%$ \\
\hline uDP4+ (all data) & $0.00 \%$ & $0.00 \%$ & $0.00 \%$ & $0.00 \%$ & $0.00 \%$ & $0.00 \%$ & $0.00 \%$ & $0.00 \%$ & oufl $100.00 \%$ & $0.00 \%$ & $0.00 \%$ & $0.00 \%$ & $0.00 \%$ & $0.00 \%$ & $0.00 \%$ & $0.00 \%$ \\
\hline DP4+ (H data) & $0.00 \%$ & $0.00 \%$ & $0.00 \%$ & $0.00 \%$ & $0.00 \%$ & $0.00 \%$ & $0.00 \%$ & $0.00 \%$ & onll100.00\% & $0.00 \%$ & $0.00 \%$ & $0.00 \%$ & $0.00 \%$ & $0.00 \%$ & $0.00 \%$ & $0.00 \%$ \\
\hline DP4+ (C data) & $0.00 \%$ & $0.00 \%$ & $0.00 \%$ & $0.00 \%$ & $0.00 \%$ & $0.00 \%$ & $0.00 \%$ & $0.00 \%$ & ofll $100.00 \%$ & $0.00 \%$ & $0.00 \%$ & $0.00 \%$ & $0.00 \%$ & $0.00 \%$ & $0.00 \%$ & $0.00 \%$ \\
\hline DP4+ (all data) & $0.00 \%$ & $0.00 \%$ & $0.00 \%$ & $0.00 \%$ & $0.00 \%$ & $0.00 \%$ & $0.00 \%$ & $0.00 \%$ & ooll100.00\% & $0.00 \%$ & $0.00 \%$ & $0.00 \%$ & $0.00 \%$ & $0.00 \%$ & $0.00 \%$ & $0.00 \%$ \\
\hline
\end{tabular}

Table S19. DP4+ results obtained using experimental data of compound 4 versus isomers 1-32

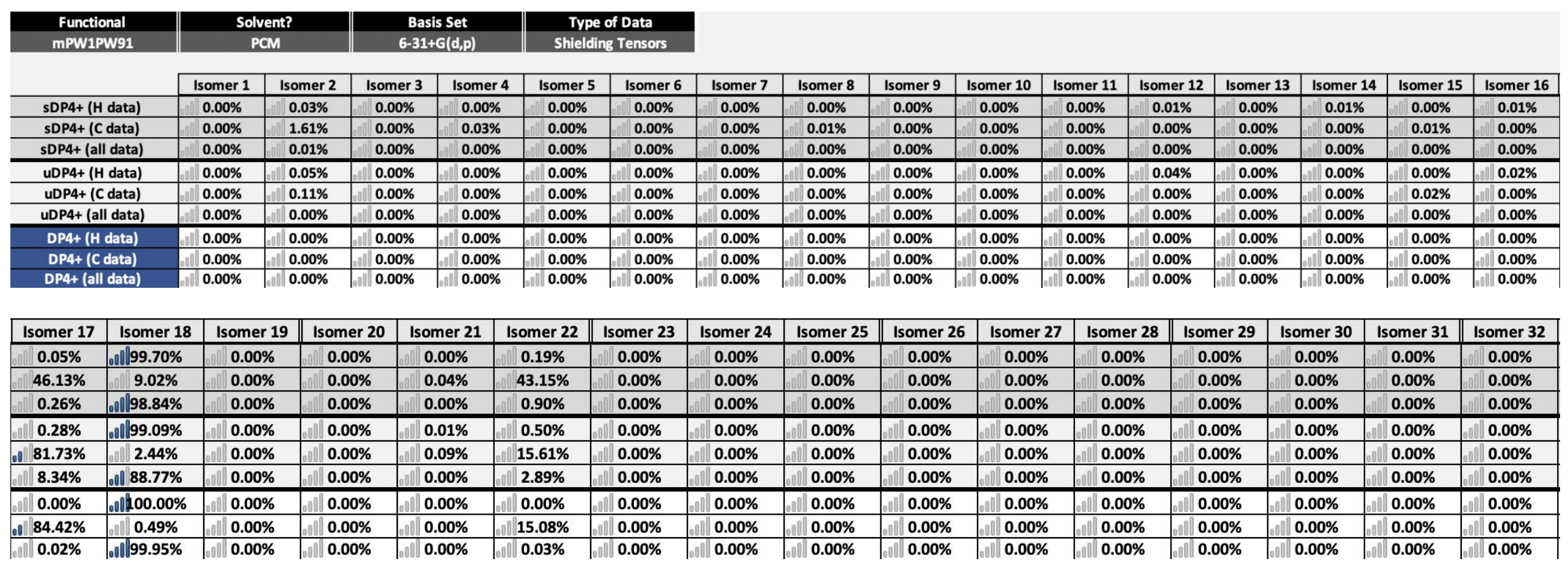


Table S20. DP4+ results obtained using experimental data of compound $\mathbf{5}$ versus isomers 1-8

\begin{tabular}{|c|c|c|c|c|c|c|c|c|}
\hline Functional & \multicolumn{2}{|c|}{ Solvent? } & \multicolumn{2}{|c|}{ Basis Set } & \multicolumn{2}{|c|}{ Type of Data } & & \\
\hline mPW1PW91 & \multicolumn{2}{|c|}{ PCM } & \multicolumn{2}{|c|}{$6-31+G(d, p)$} & \multicolumn{2}{|c|}{ Shielding Tensors } & & \\
\hline & Isomer 1 & Isomer 2 & Isomer 3 & Isomer 4 & Isomer 5 & Isomer 6 & Isomer 7 & Isomer 8 \\
\hline sDP4+ (H data) & $0.04 \%$ & $4.31 \%$ & $0.00 \%$ & ${ }_{000} \| 95.55 \%$ & $0.00 \%$ & $0.06 \%$ & $0.00 \%$ & $0.04 \%$ \\
\hline sDP4+ (C data) & $1.60 \%$ & 013.98\% & $0.07 \%$ & $12.83 \%$ & $0.03 \%$ & $11.40 \%$ & $0.04 \%$ & $0.05 \%$ \\
\hline sDP4+ (all data) & $0.00 \%$ & $20.62 \%$ & $0.00 \%$ & 79.33\% & $0.00 \%$ & $0.05 \%$ & $0.00 \%$ & $0.00 \%$ \\
\hline uDP4+ (H data) & $0.00 \%$ & $0.58 \%$ & $0.00 \%$ & ool $\int 97.99 \%$ & $0.01 \%$ & $1.37 \%$ & $0.00 \%$ & $0.05 \%$ \\
\hline uDP4+ (C data) & $11.27 \%$ & $62.96 \%$ & $0.88 \%$ & $16.89 \%$ & $0.35 \%$ & $7.24 \%$ & $0.35 \%$ & $0.05 \%$ \\
\hline uDP4+ (all data) & $0.00 \%$ & ofl $2.15 \%$ & $0.00 \%$ & ${ }_{000} \| 97.27 \%$ & $0.00 \%$ & $0.58 \%$ & $0.00 \%$ & $0.00 \%$ \\
\hline DP4+ (H data) & $0.00 \%$ & $0.03 \%$ & $0.00 \%$ & ol] $999.97 \%$ & $0.00 \%$ & $0.00 \%$ & $0.00 \%$ & $0.00 \%$ \\
\hline DP4+ (C data) & $0.36 \%$ & oll $93.62 \%$ & $0.00 \%$ & 10.36\% & $0.00 \%$ & $1.66 \%$ & $0.00 \%$ & $0.00 \%$ \\
\hline DP4+ (all data) & $0.00 \%$ & o00 $0.57 \%$ & $0.00 \%$ & ${ }_{000} 0 \mid 99.43 \%$ & $0.00 \%$ & $0.00 \%$ & $0.00 \%$ & $0.00 \%$ \\
\hline
\end{tabular}

Table S21. DP4+ results obtained using experimental data of compound isoelisabethatriene (vacuum) versus isomers 1-4

\begin{tabular}{|c|c|c|c|c|c|c|}
\hline Functional & \multicolumn{2}{|c|}{ Solvent? } & \multicolumn{2}{|c|}{ Basis Set } & \multicolumn{2}{|c|}{ Type of Data } \\
\hline mPW1PW91 & \multicolumn{2}{|c|}{ PCM } & \multicolumn{2}{|c|}{$6-31+G(d, p)$} & \multicolumn{2}{|c|}{ Shielding Tensors } \\
\hline & Isomer 1 & Isomer 2 & Isomer 3 & Isomer 4 & Isomer 5 & Isomer 6 \\
\hline sDP4+ (H data) & $0.01 \%$ & ol $92.78 \%$ & $7.20 \%$ & $0.01 \%$ & - & - \\
\hline sDP4+ (C data) & $0.00 \%$ & ol $\int 99.23 \%$ & $0.47 \%$ & $0.30 \%$ & - & - \\
\hline sDP4+ (all data) & $0.00 \%$ & ${ }_{0} 0$ ل $99.96 \%$ & $0.04 \%$ & $0.00 \%$ & - & - \\
\hline uDP4+ (H data) & $0.23 \%$ & $81.23 \%$ & $18.44 \%$ & $0.10 \%$ & - & - \\
\hline uDP4+ (C data) & $0.00 \%$ & ond $99.76 \%$ & $0.14 \%$ & $0.09 \%$ & - & - \\
\hline uDP4+ (all data) & $0.00 \%$ & ol $\| 99.97 \%$ & $0.03 \%$ & $0.00 \%$ & - & - \\
\hline DP4+ (H data) & $0.00 \%$ & ${ }_{0} 0 \| 98.27 \%$ & $1.73 \%$ & $0.00 \%$ & - & - \\
\hline DP4+ (C data) & $0.00 \%$ & oult $100.00 \%$ & $0.00 \%$ & $0.00 \%$ & - & - \\
\hline DP4+ (all data) & $0.00 \%$ & onlt $100.00 \%$ & $0.00 \%$ & $0.00 \%$ & - & - \\
\hline
\end{tabular}


Table S22. DP4+ results obtained using experimental data of compound isoelisabethatriene (benzene) versus isomers 1-4

\begin{tabular}{|c|c|c|c|c|c|c|}
\hline Functional & \multicolumn{2}{|c|}{ Solvent? } & \multicolumn{2}{|c|}{ Basis Set } & \multicolumn{2}{|c|}{ Type of Data } \\
\hline \multirow{2}{*}{ mPW1PW91 } & \multicolumn{2}{|c|}{ PCM } & \multicolumn{2}{|c|}{$6-31+G(d, p)$} & \multicolumn{2}{|c|}{ Shielding Tensors } \\
\hline & Isomer 1 & Isomer 2 & Isomer 3 & Isomer 4 & Isomer 5 & Isomer 6 \\
\hline sDP4+ (H data) & $0.01 \%$ & . $89.03 \%$ & $10.95 \%$ & $0.01 \%$ & - & - \\
\hline sDP4+ (C data) & $0.00 \%$ & ${ }_{0} 00 \mid 99.03 \%$ & $0.48 \%$ & $0.49 \%$ & - & - \\
\hline sDP4+ (all data) & $0.00 \%$ & olol $99.94 \%$ & $0.06 \%$ & $0.00 \%$ & - & - \\
\hline uDP4+ (H data) & $0.22 \%$ & $017.64 \%$ & $22.07 \%$ & $0.07 \%$ & - & - \\
\hline uDP4+ (C data) & $0.00 \%$ & ol0 $999.61 \%$ & $0.17 \%$ & $0.22 \%$ & - & - \\
\hline uDP4+ (all data) & $0.00 \%$ & old $999.95 \%$ & $0.05 \%$ & $0.00 \%$ & - & - \\
\hline DP4+ (H data) & $0.00 \%$ & ofll $996.62 \%$ & $3.38 \%$ & $0.00 \%$ & - & - \\
\hline DP4+ (C data) & $0.00 \%$ & ollt $00.00 \%$ & $0.00 \%$ & $0.00 \%$ & - & - \\
\hline DP4+ (all data) & $0.00 \%$ & onlto0.00\% & $0.00 \%$ & $0.00 \%$ & - & - \\
\hline
\end{tabular}




\subsection{Correlation plots of compounds $1-5$ and isoelisabethatriene}

Figure S75. Compound 1 correlation plots between ${ }^{13} \mathrm{C}$ isotropic magnetic shielding values computed at the $\mathrm{PCM} / \mathrm{mPW} 1 \mathrm{PW} 91 / 6-31+\mathrm{G}^{* *}$ level of theory and experimental ${ }^{13} \mathrm{C}$ chemical shifts for isomers 1-8

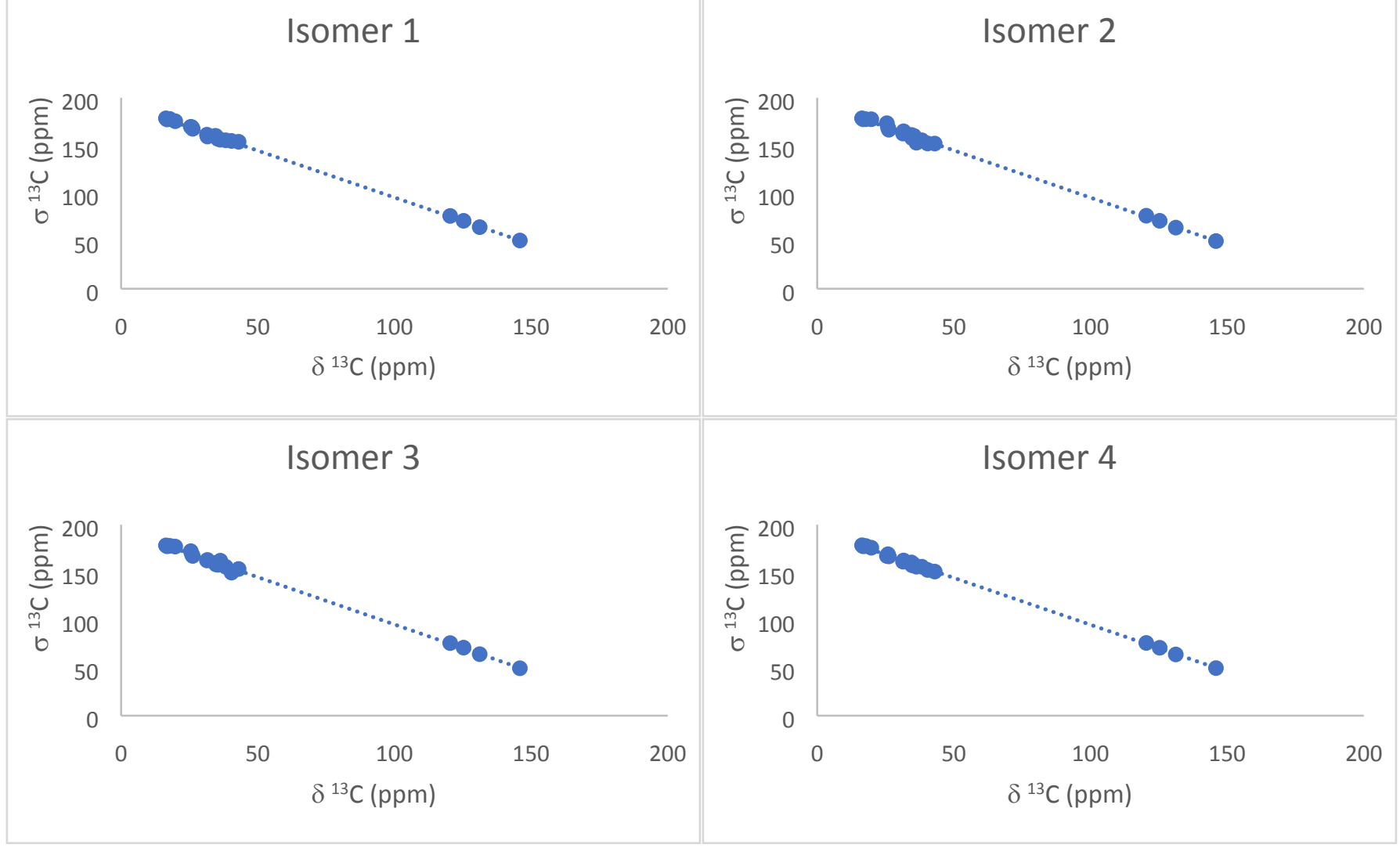




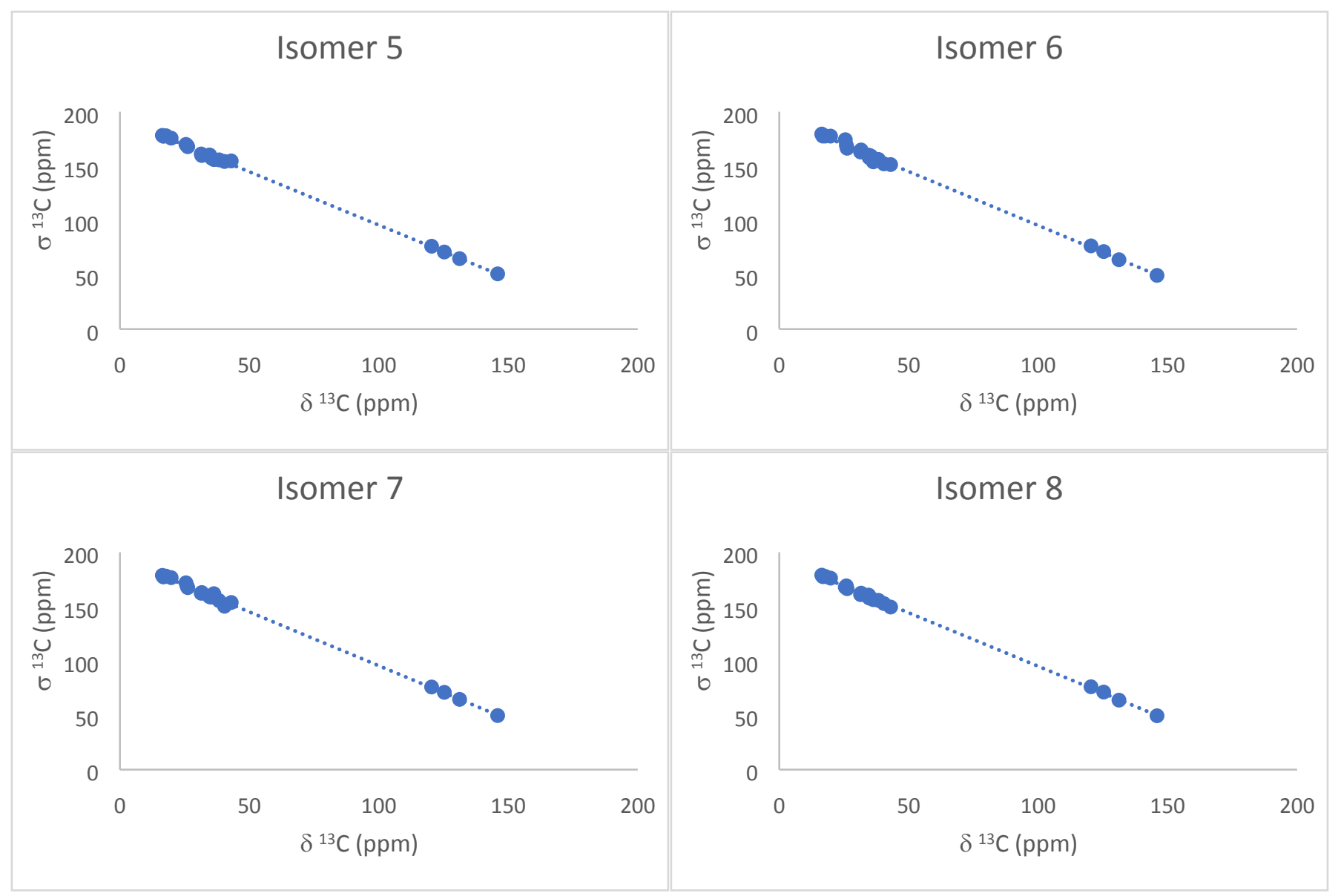


Figure S76. Compound 1 correlation plots between ${ }^{1} \mathrm{H}$ isotropic magnetic shielding values computed at the PCM/mPW1PW91/6-31+G** level of theory and experimental ${ }^{1} \mathrm{H}$ chemical shifts for isomers $1-8$

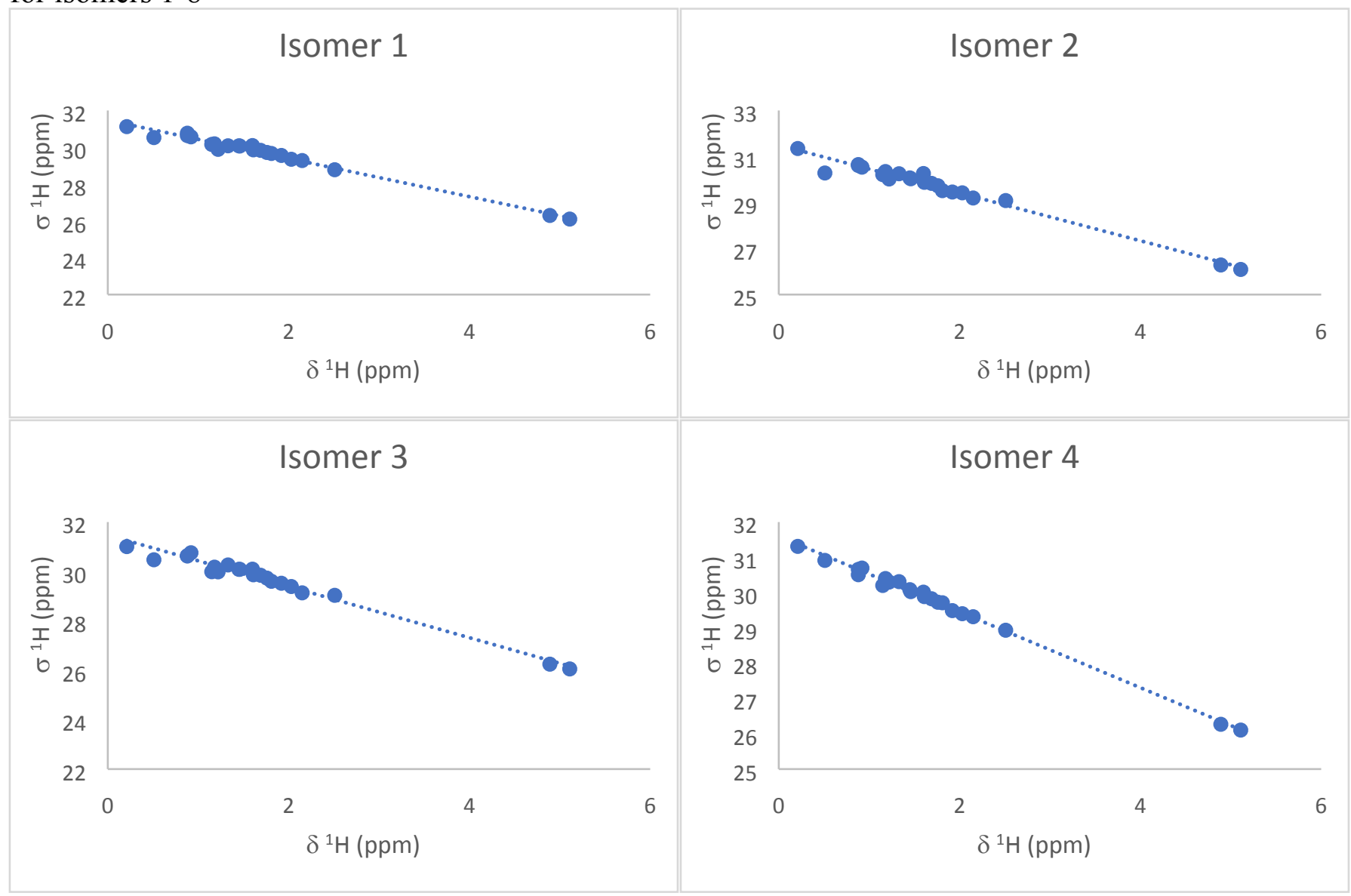




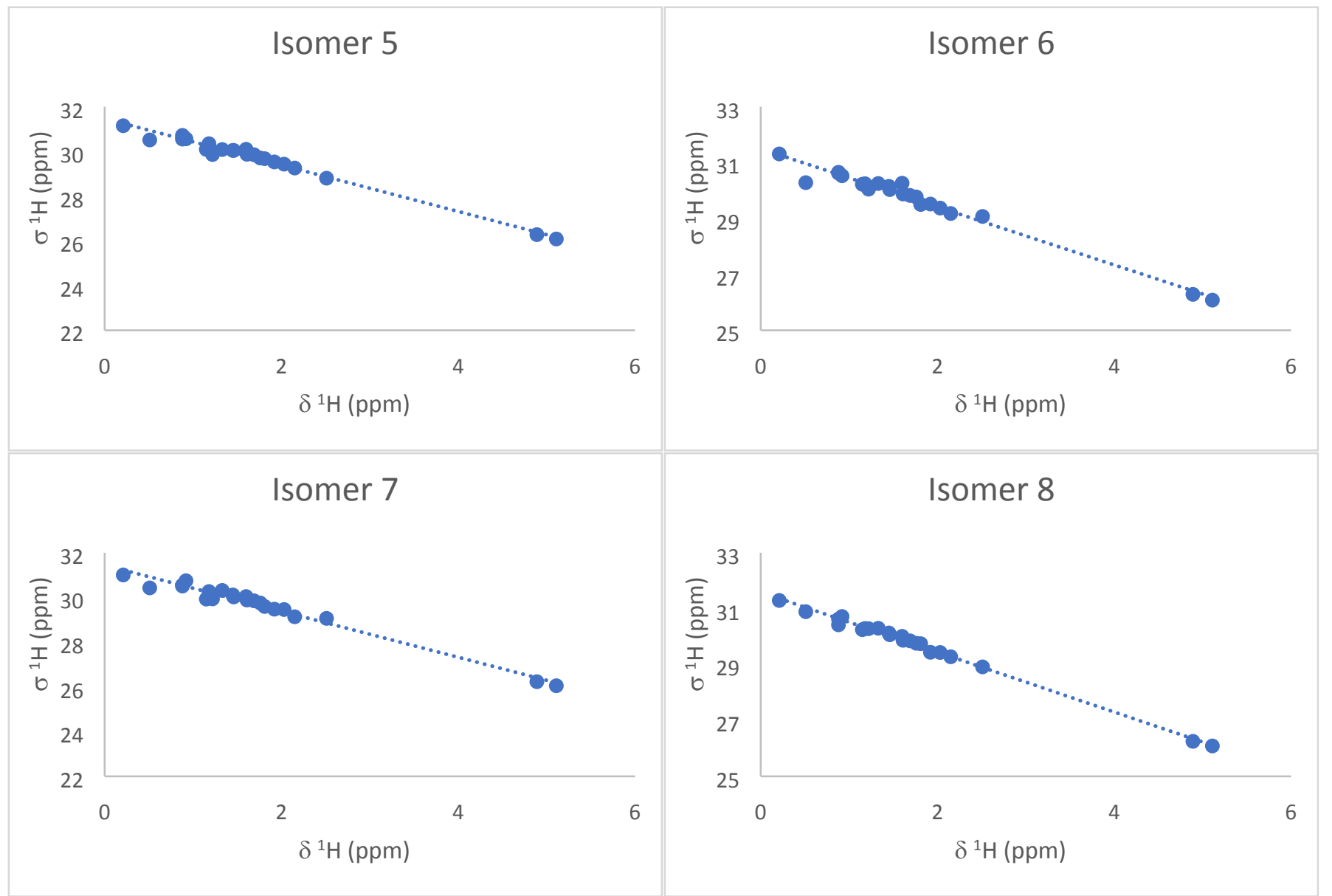

Figure S77. Compound 2 correlation plots between ${ }^{13} \mathrm{C}$ isotropic magnetic shielding values computed at the $\mathrm{PCM} / \mathrm{mPW} 1 \mathrm{PW} 91 / 6-31+\mathrm{G}^{* *}$ level of theory and experimental ${ }^{13} \mathrm{C}$ chemical shifts for isomers $1-8$

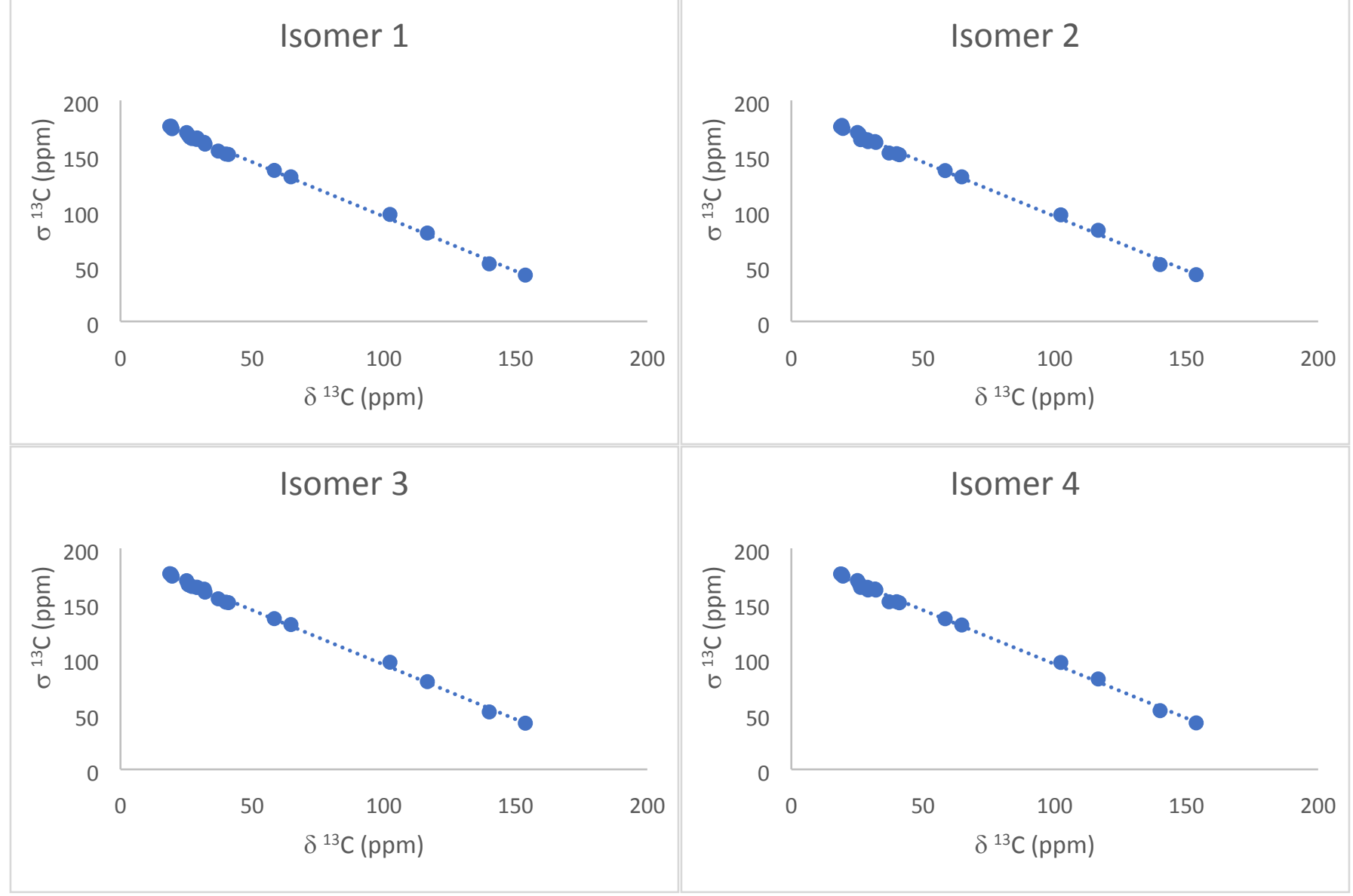




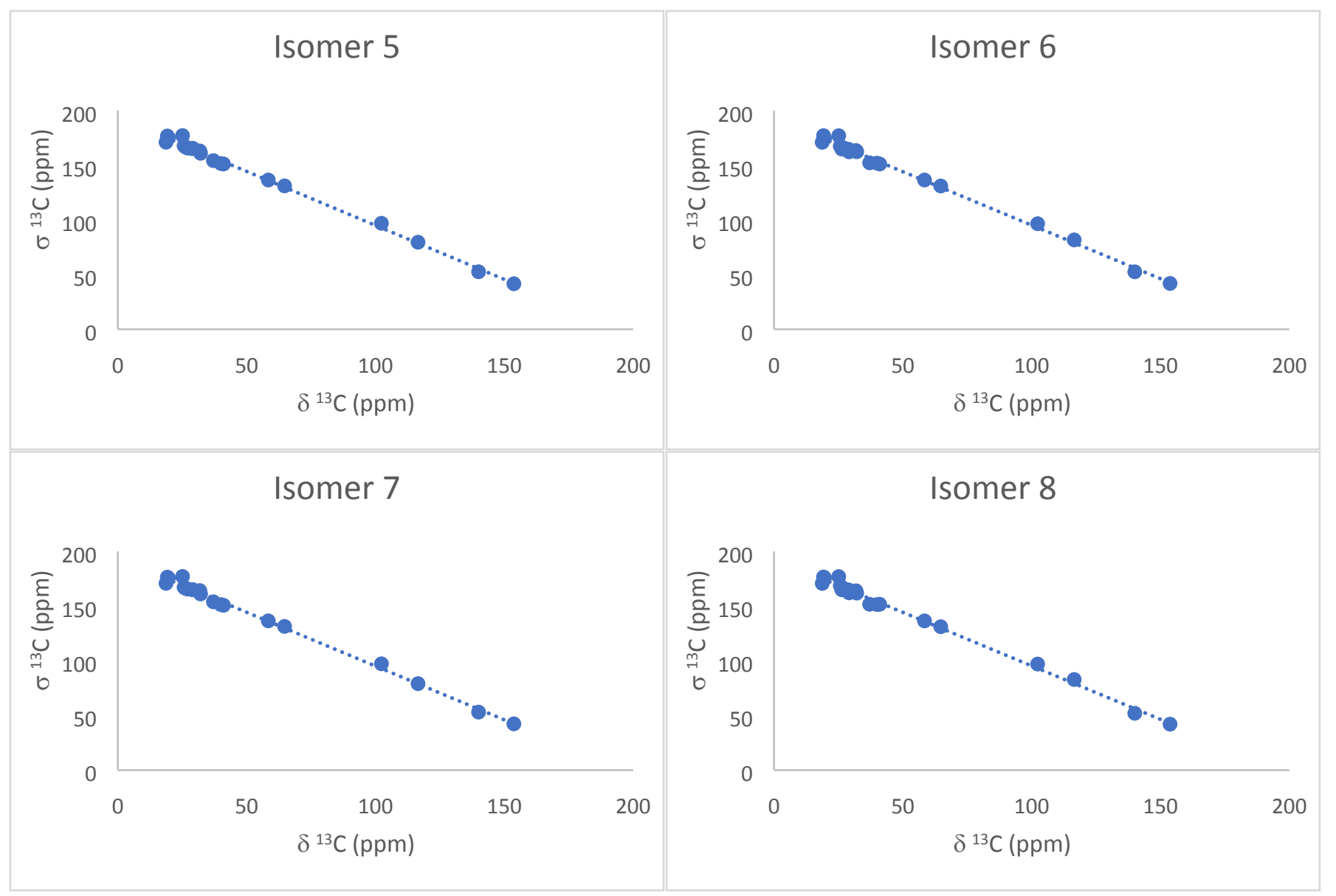


Figure S78. Compound 2 correlation plots between ${ }^{1} \mathrm{H}$ isotropic magnetic shielding values computed at the $\mathrm{PCM} / \mathrm{mPW} 1 \mathrm{PW} 91 / 6-31+\mathrm{G}^{* *}$ level of theory and experimental ${ }^{1} \mathrm{H}$ chemical shifts for isomers $1-8$

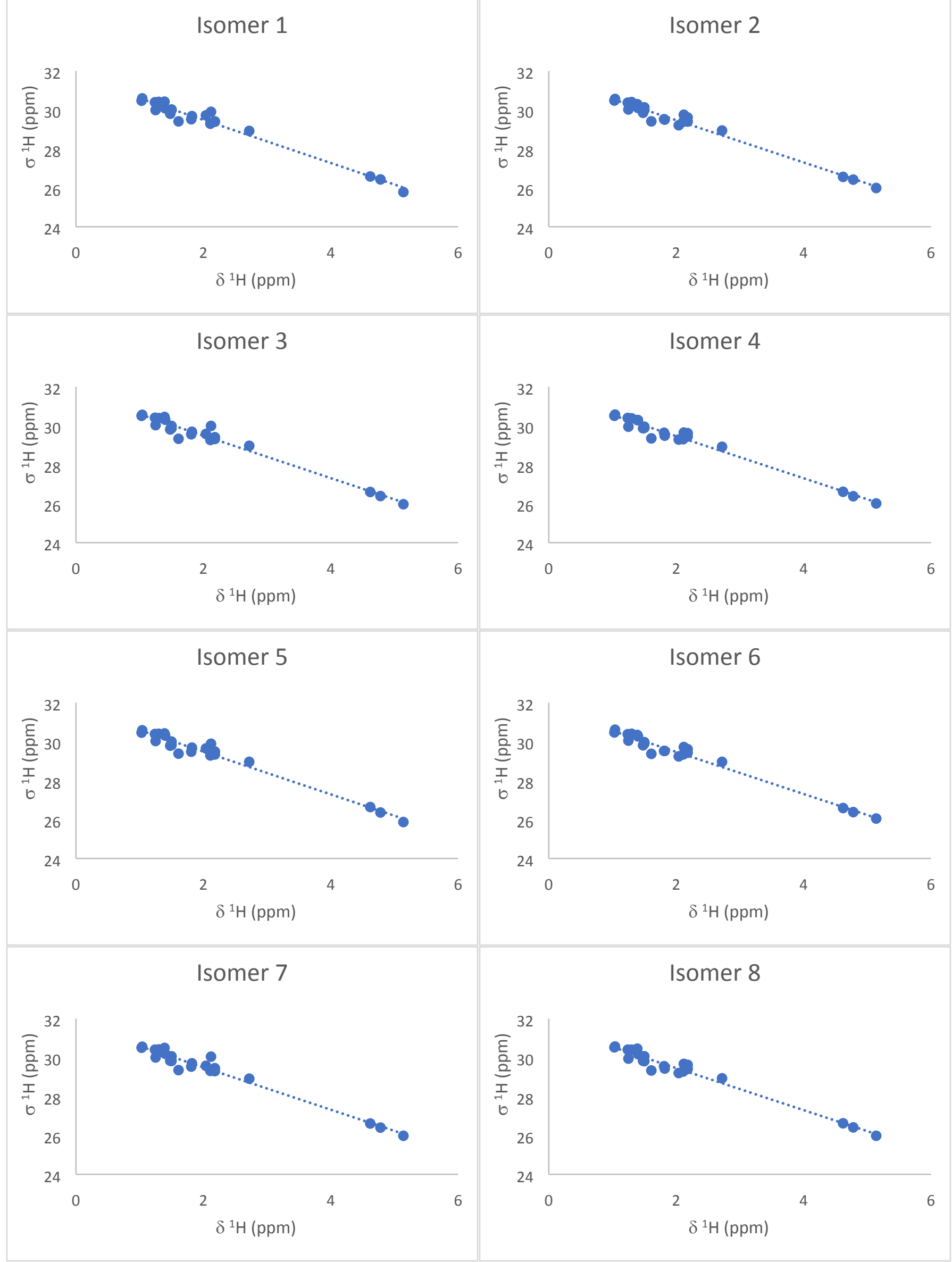


Figure S79. Compound 3 correlation plots between ${ }^{13} \mathrm{C}$ isotropic magnetic shielding values computed at the $\mathrm{PCM} / \mathrm{mPW} 1 \mathrm{PW} 91 / 6-31+\mathrm{G}^{* *}$ level of theory and experimental ${ }^{13} \mathrm{C}$ chemical shifts for isomers 1-16

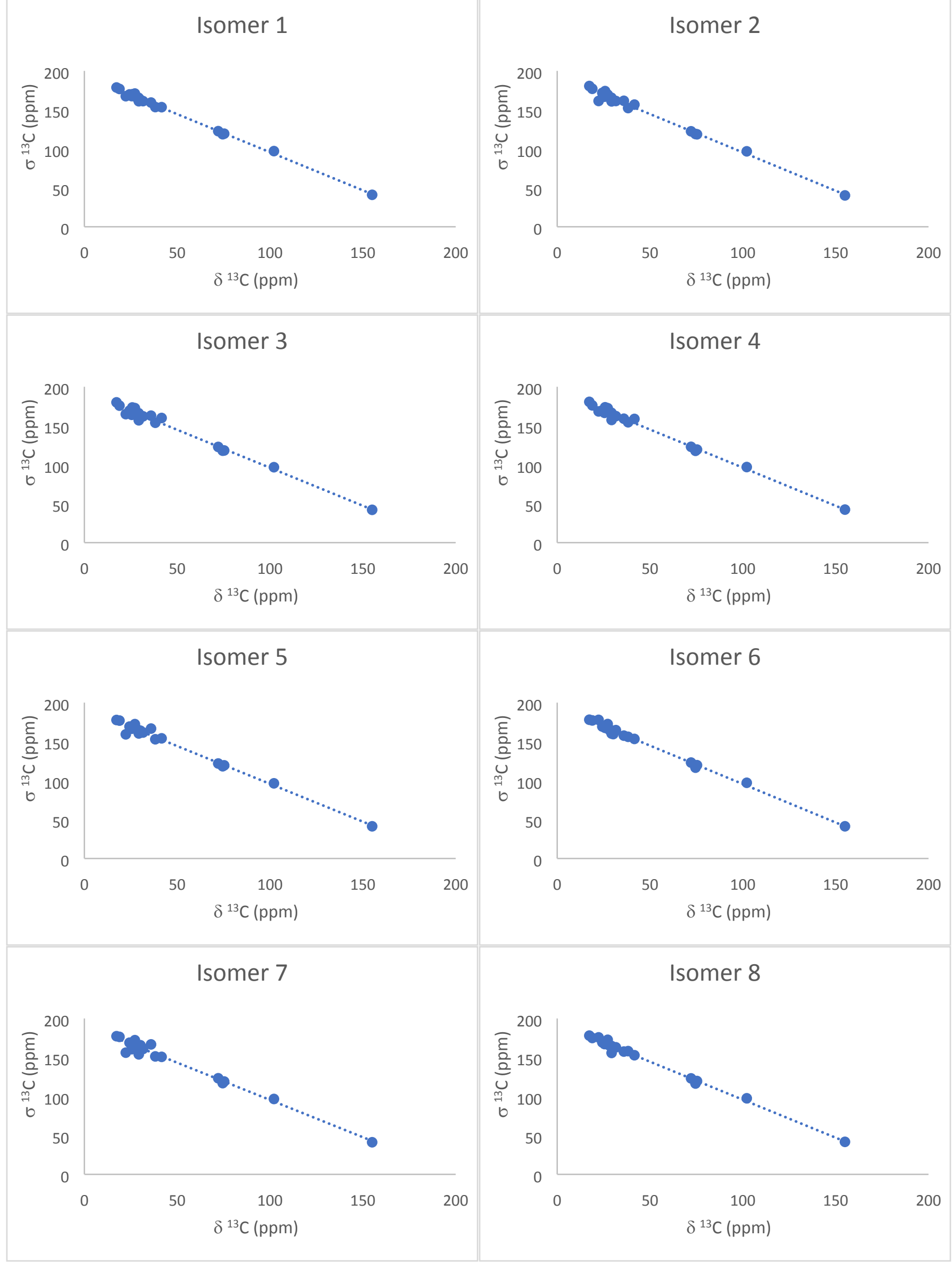




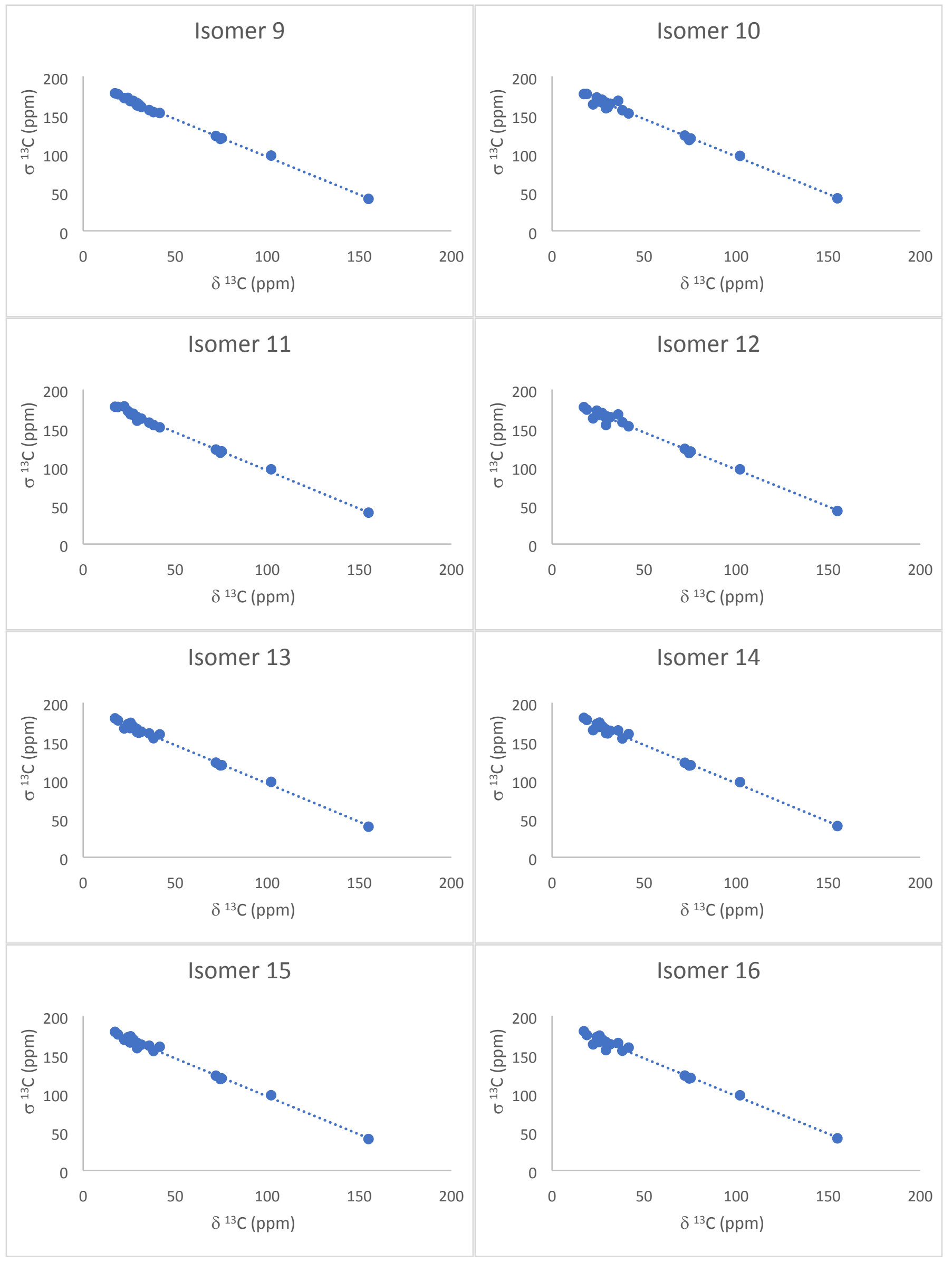


Figure S80. Compound 3 correlation plots between ${ }^{1} \mathrm{H}$ isotropic magnetic shielding values computed at the $\mathrm{PCM} / \mathrm{mPW} 1 \mathrm{PW} 91 / 6-31+\mathrm{G}^{* *}$ level of theory and experimental ${ }^{1} \mathrm{H}$ chemical shifts for isomers 1-16

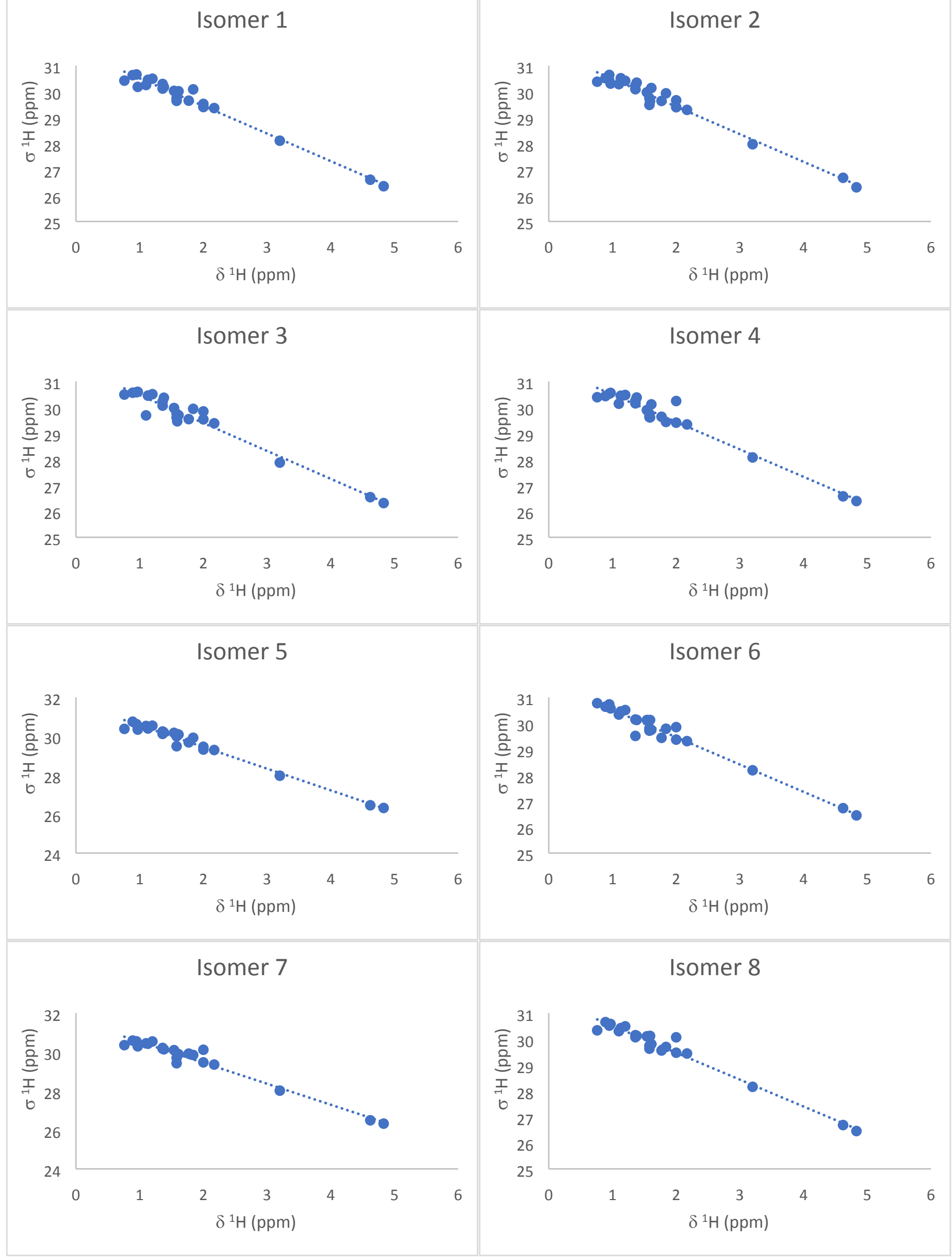




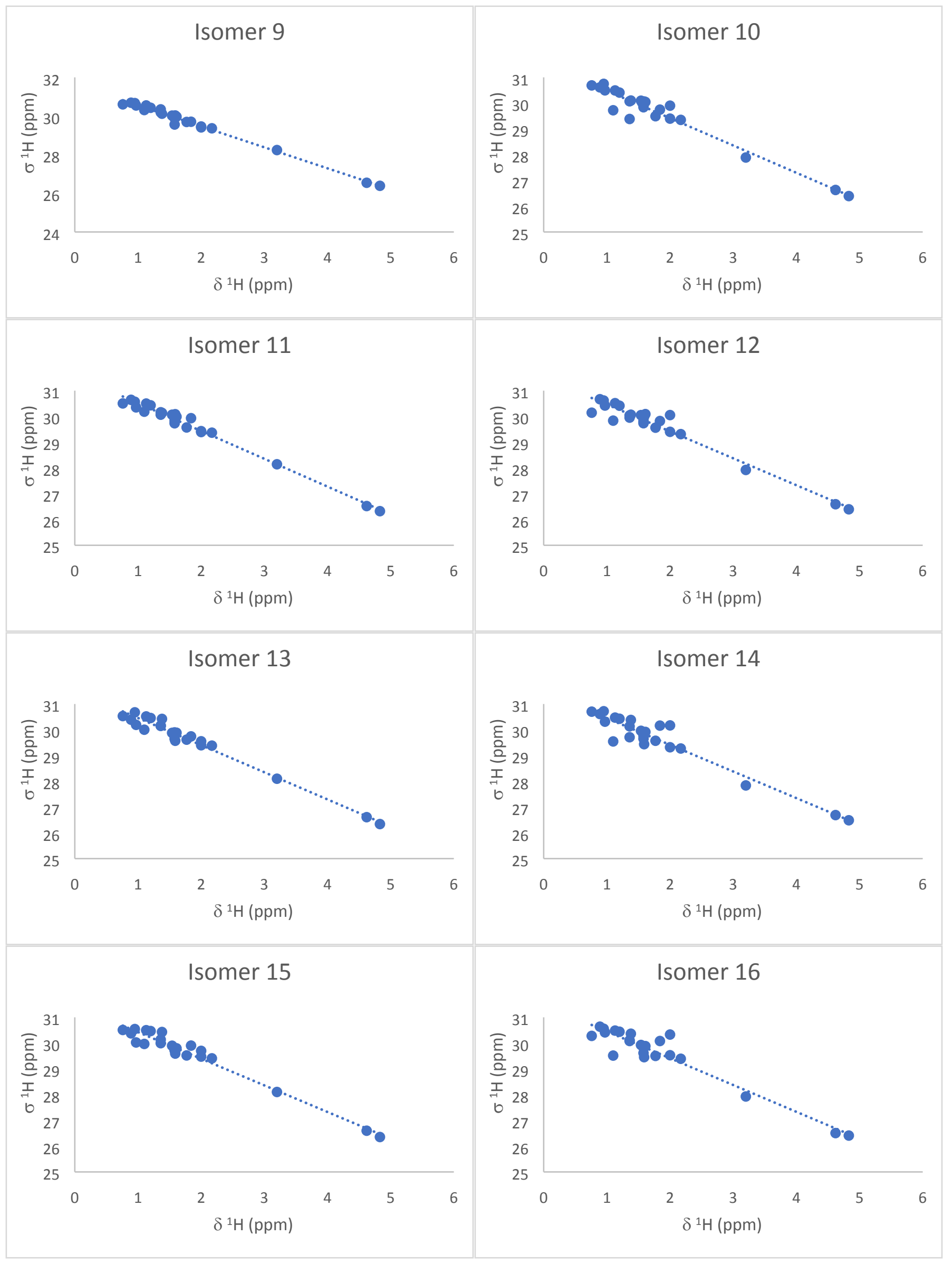


Figure S81. Compound 4 correlation plots between ${ }^{13} \mathrm{C}$ isotropic magnetic shielding values computed at the $\mathrm{PCM} / \mathrm{mPW} 1 \mathrm{PW} 91 / 6-31+\mathrm{G}^{* *}$ level of theory and experimental ${ }^{13} \mathrm{C}$ chemical shifts for isomers 1-32

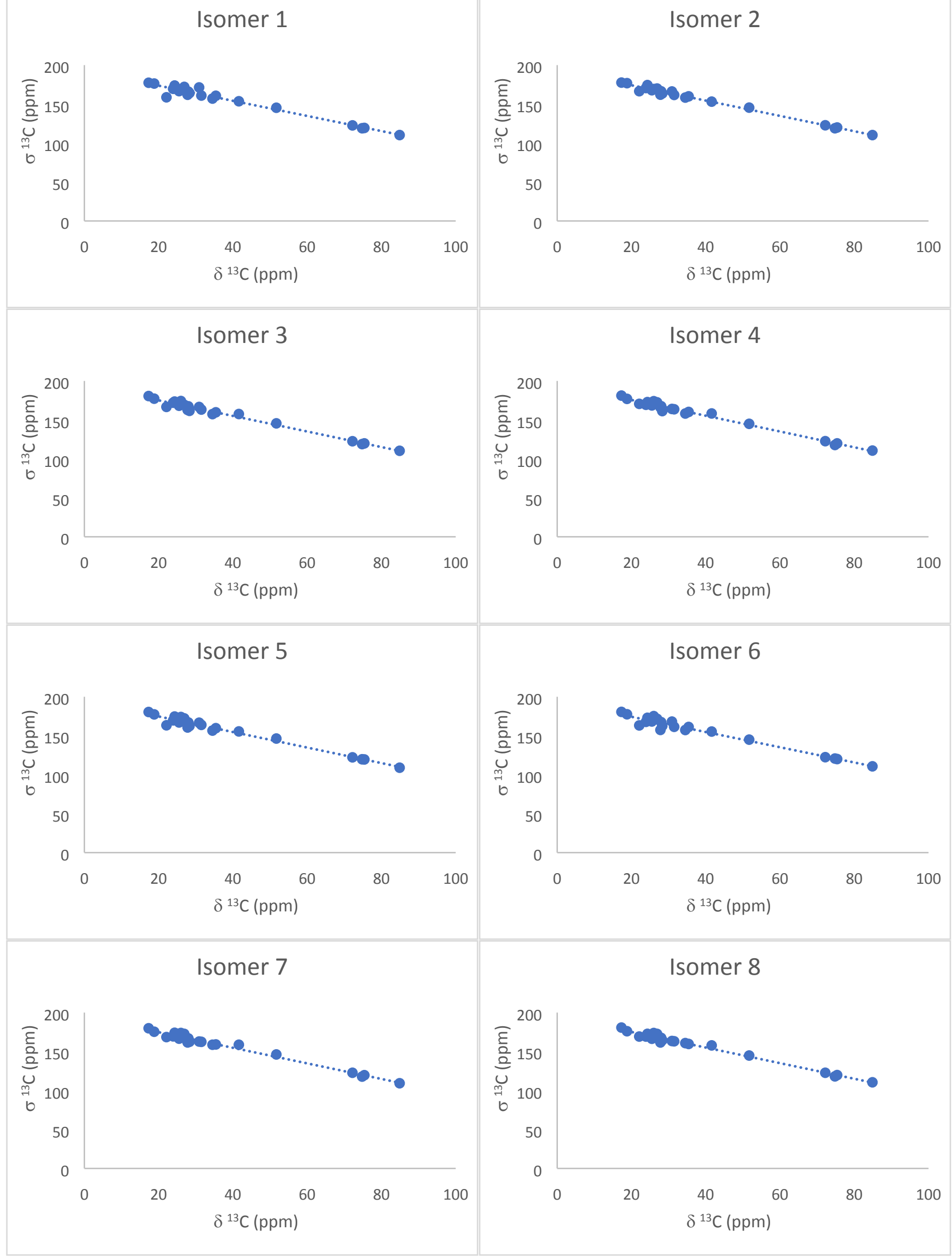




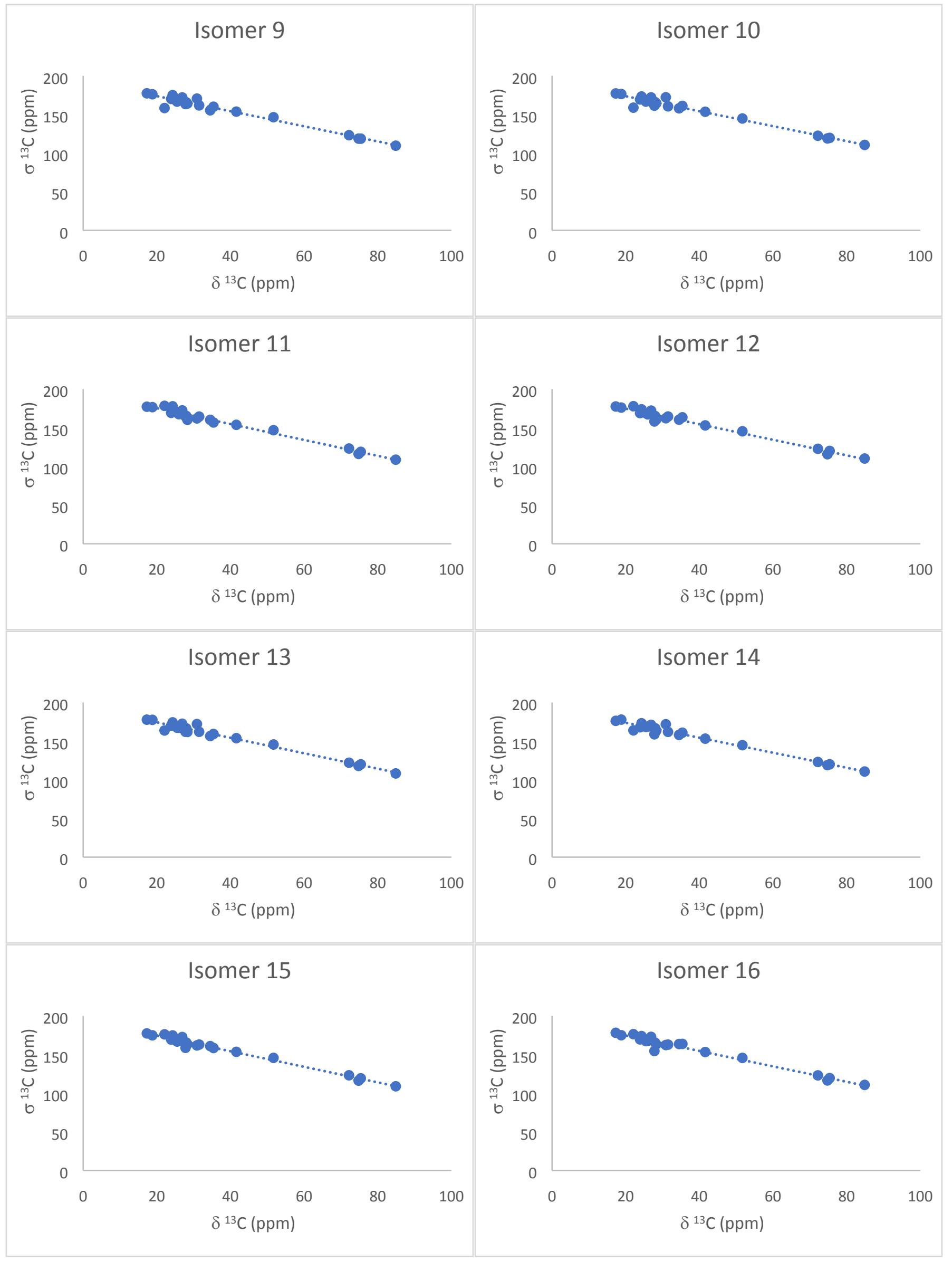




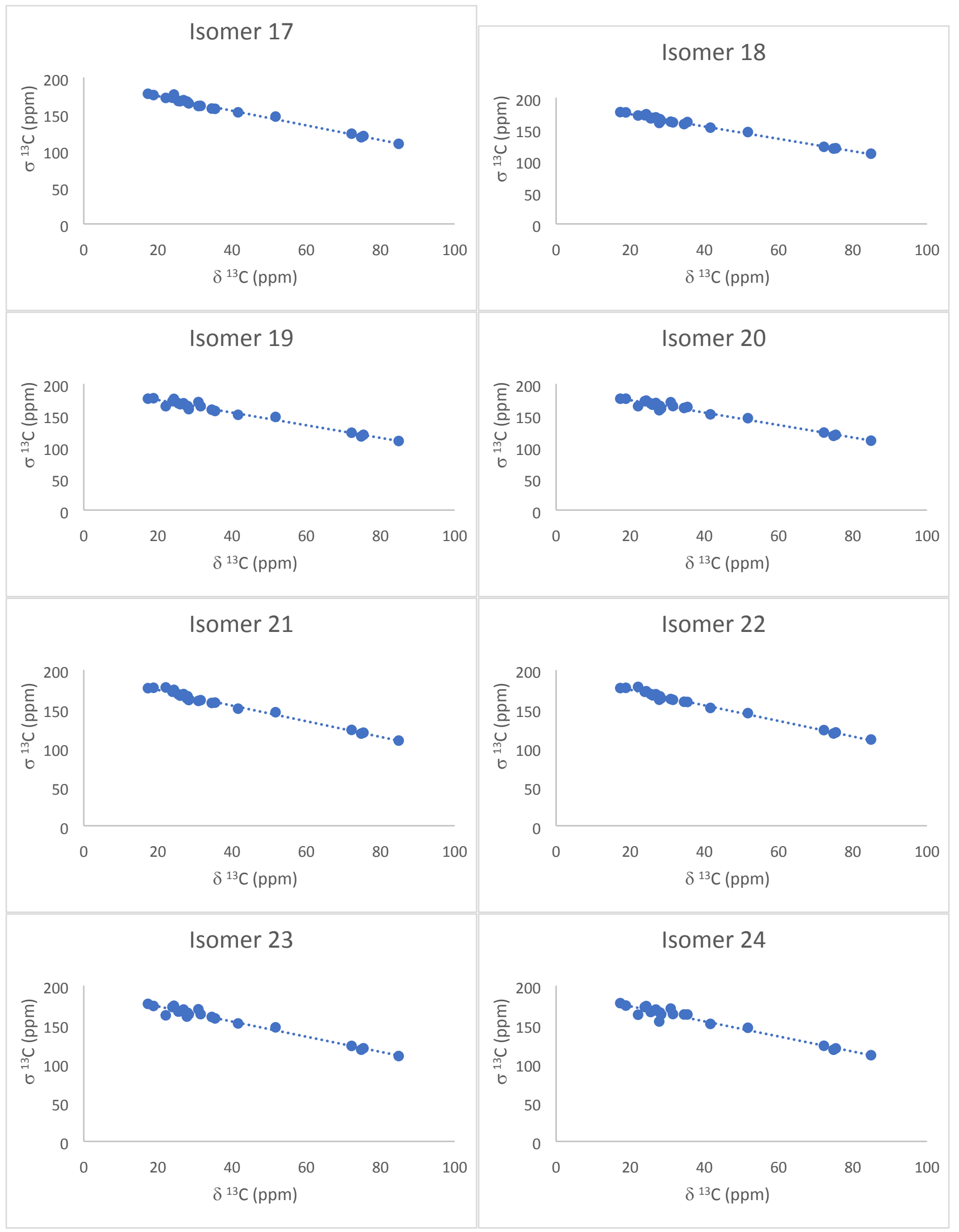




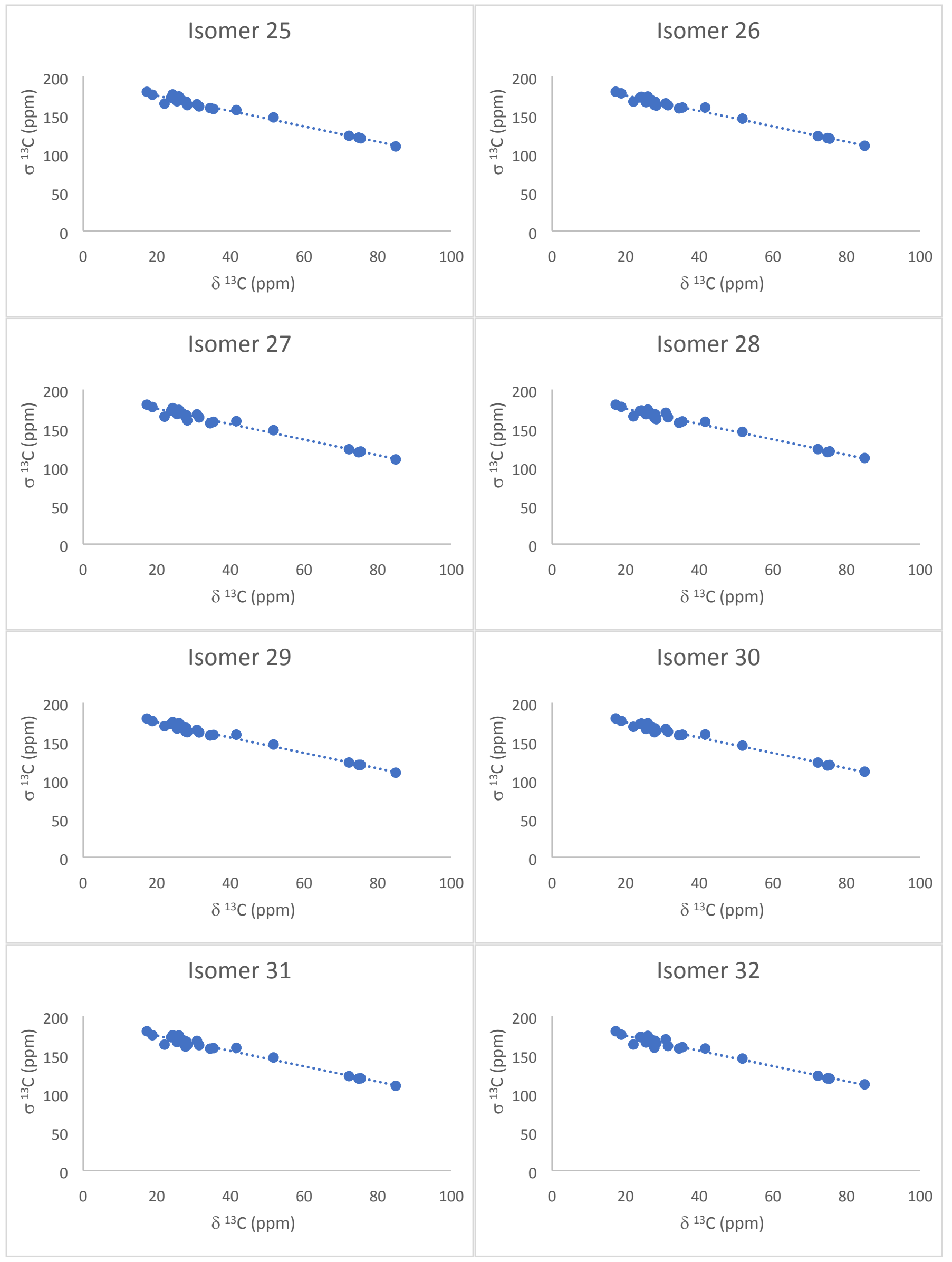


Figure S82. Compound 4 correlation plots between ${ }^{1} \mathrm{H}$ isotropic magnetic shielding values computed at the $\mathrm{PCM} / \mathrm{mPW} 1 \mathrm{PW} 91 / 6-31+\mathrm{G}^{* *}$ level of theory and experimental ${ }^{1} \mathrm{H}$ chemical shifts for isomers 1-32

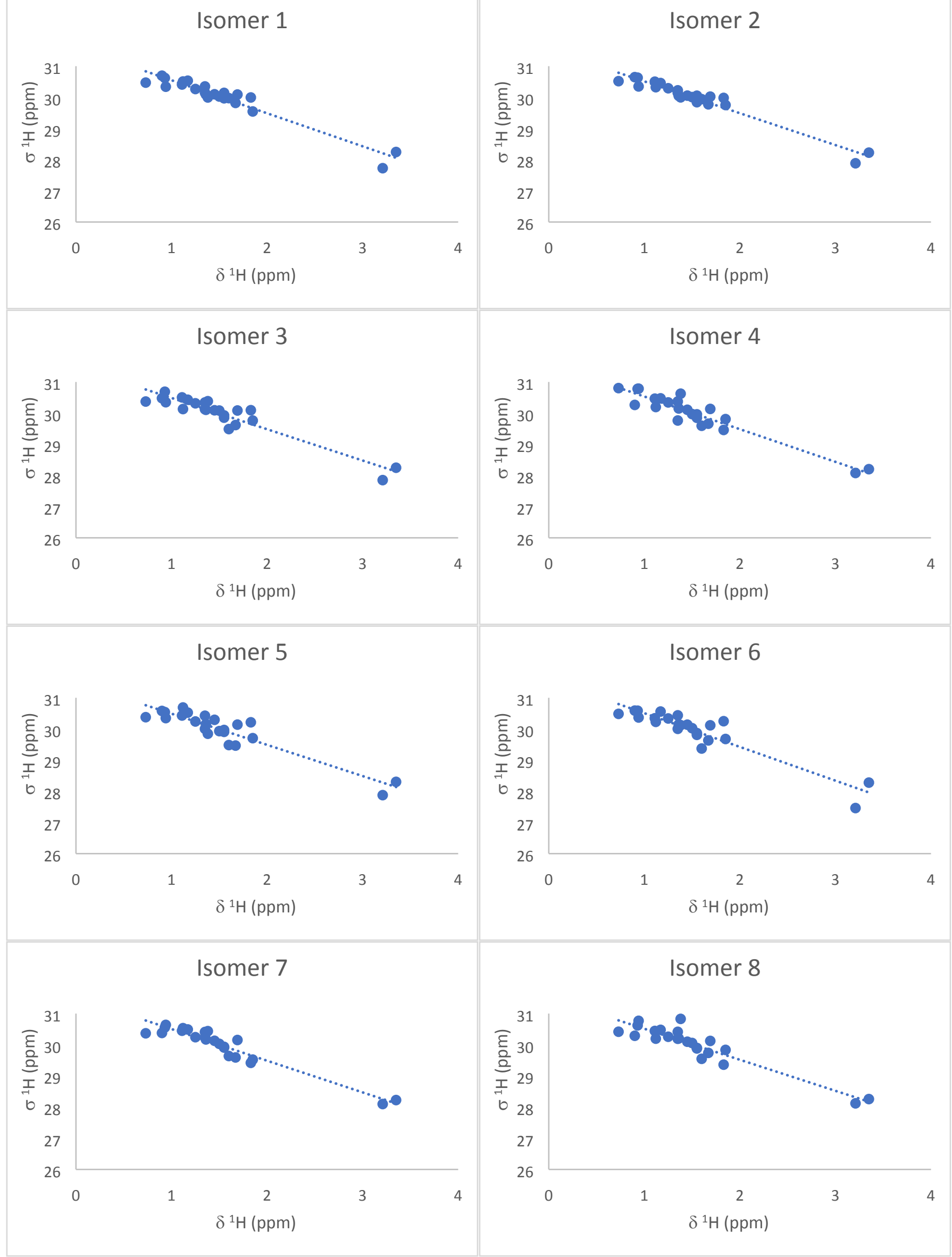




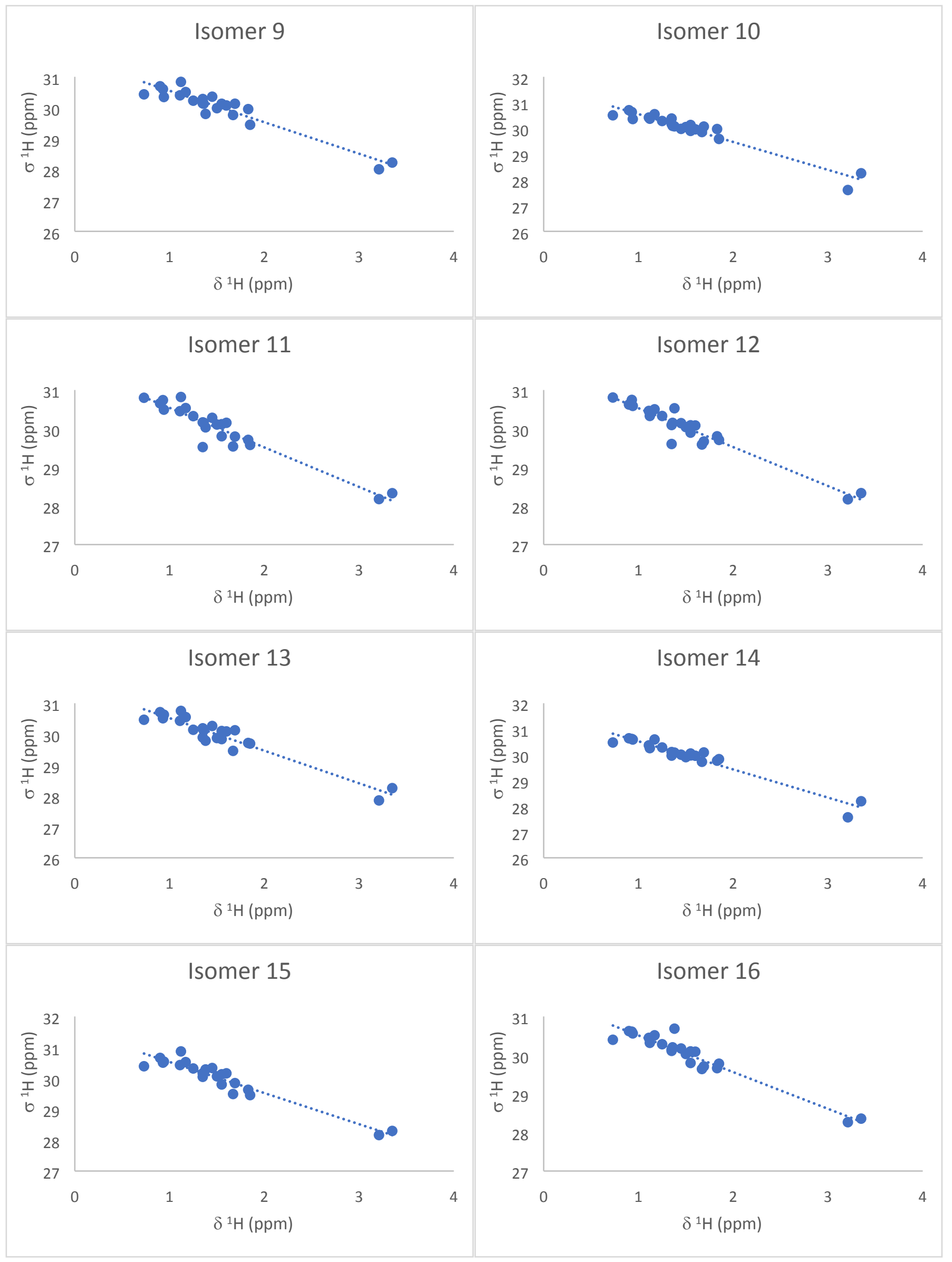




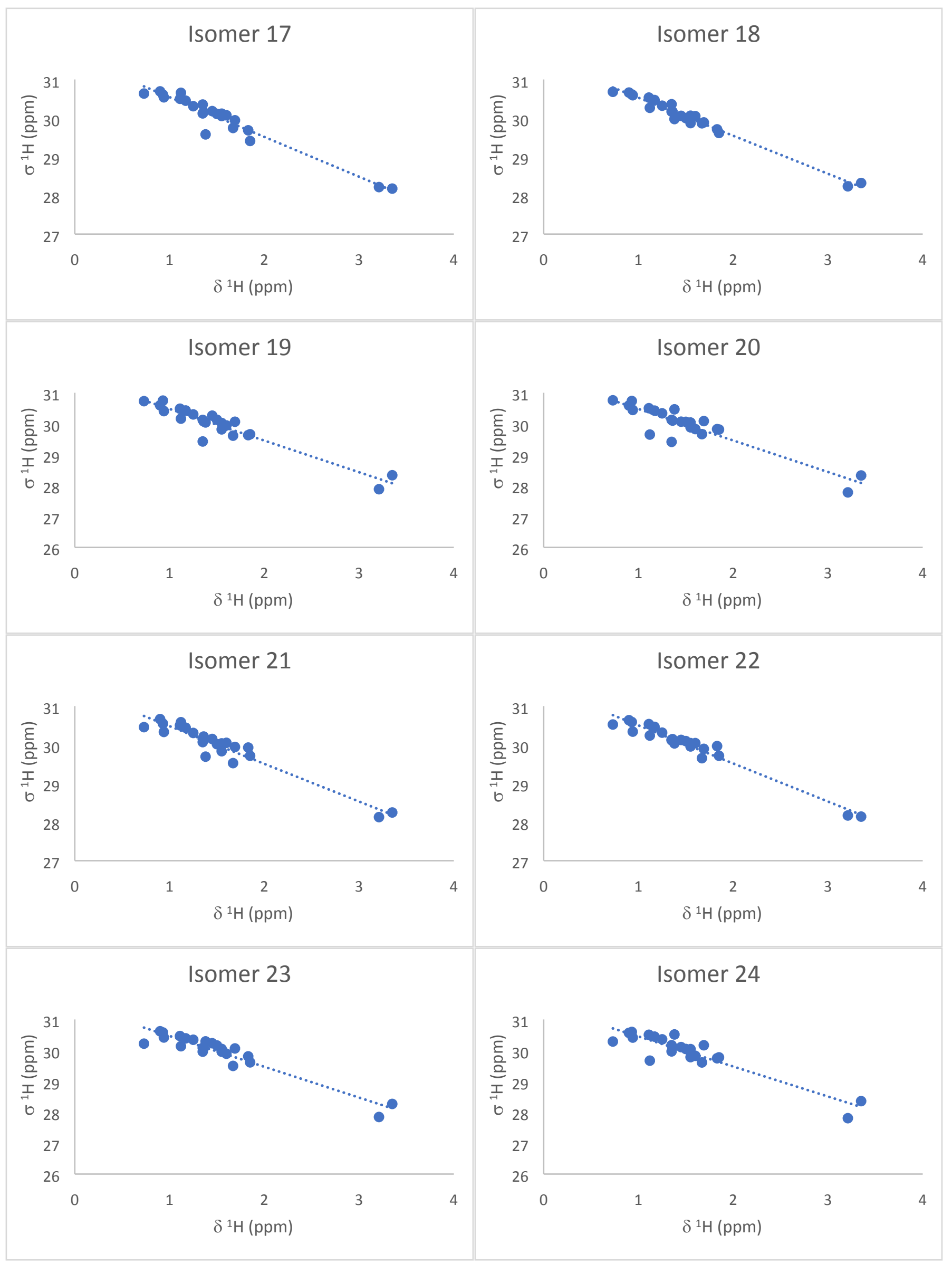




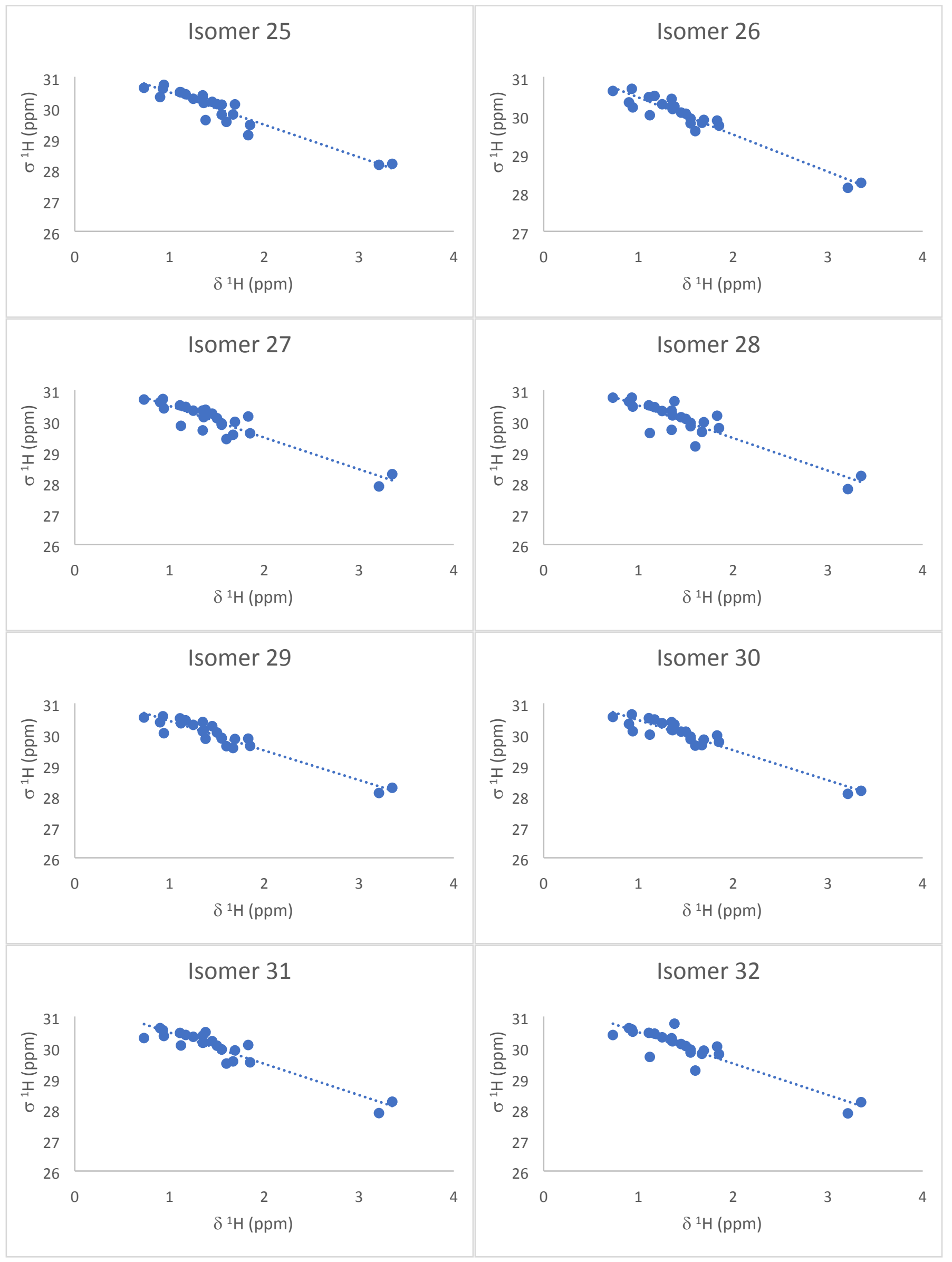


Figure S83. Compound 5 correlation plots between ${ }^{13} \mathrm{C}$ isotropic magnetic shielding values computed at the $\mathrm{PCM} / \mathrm{mPW} 1 \mathrm{PW} 91 / 6-31+\mathrm{G}^{* *}$ level of theory and experimental ${ }^{13} \mathrm{C}$ chemical shifts for isomers 1-8

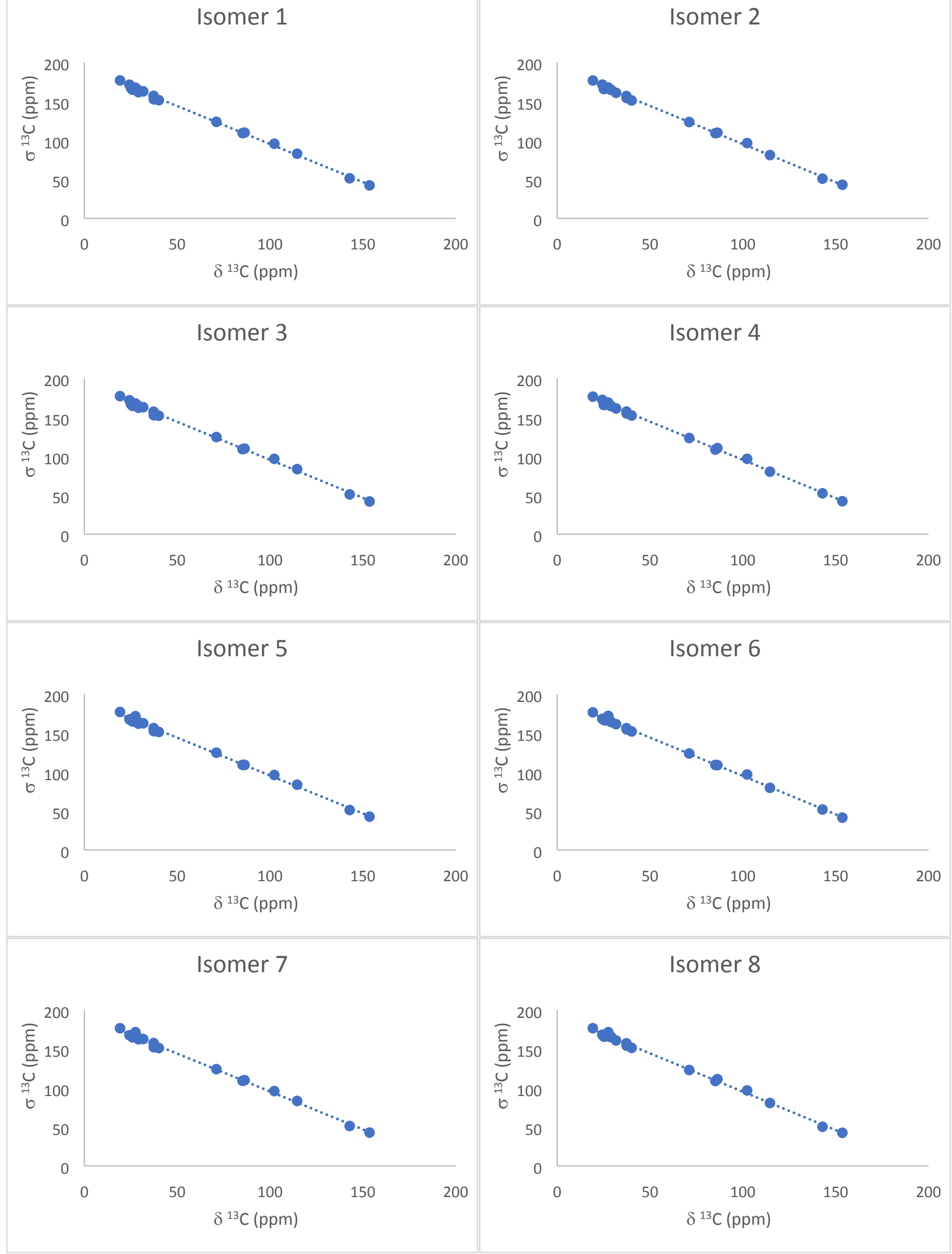


Figure S84. Compound 5 correlation plots between ${ }^{1} \mathrm{H}$ isotropic magnetic shielding values computed at the $\mathrm{PCM} / \mathrm{mPW} 1 \mathrm{PW} 91 / 6-31+\mathrm{G}^{* *}$ level of theory and experimental ${ }^{1} \mathrm{H}$ chemical shifts for isomers $1-8$

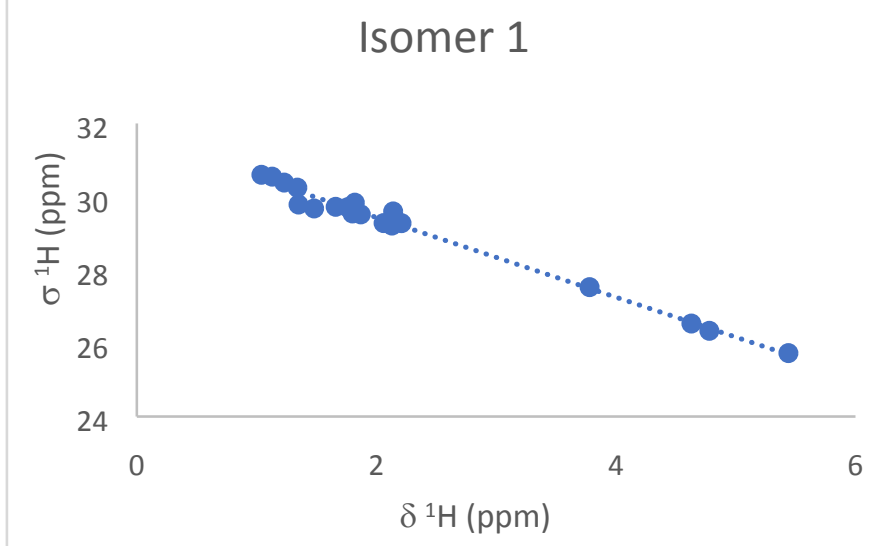

Isomer 2
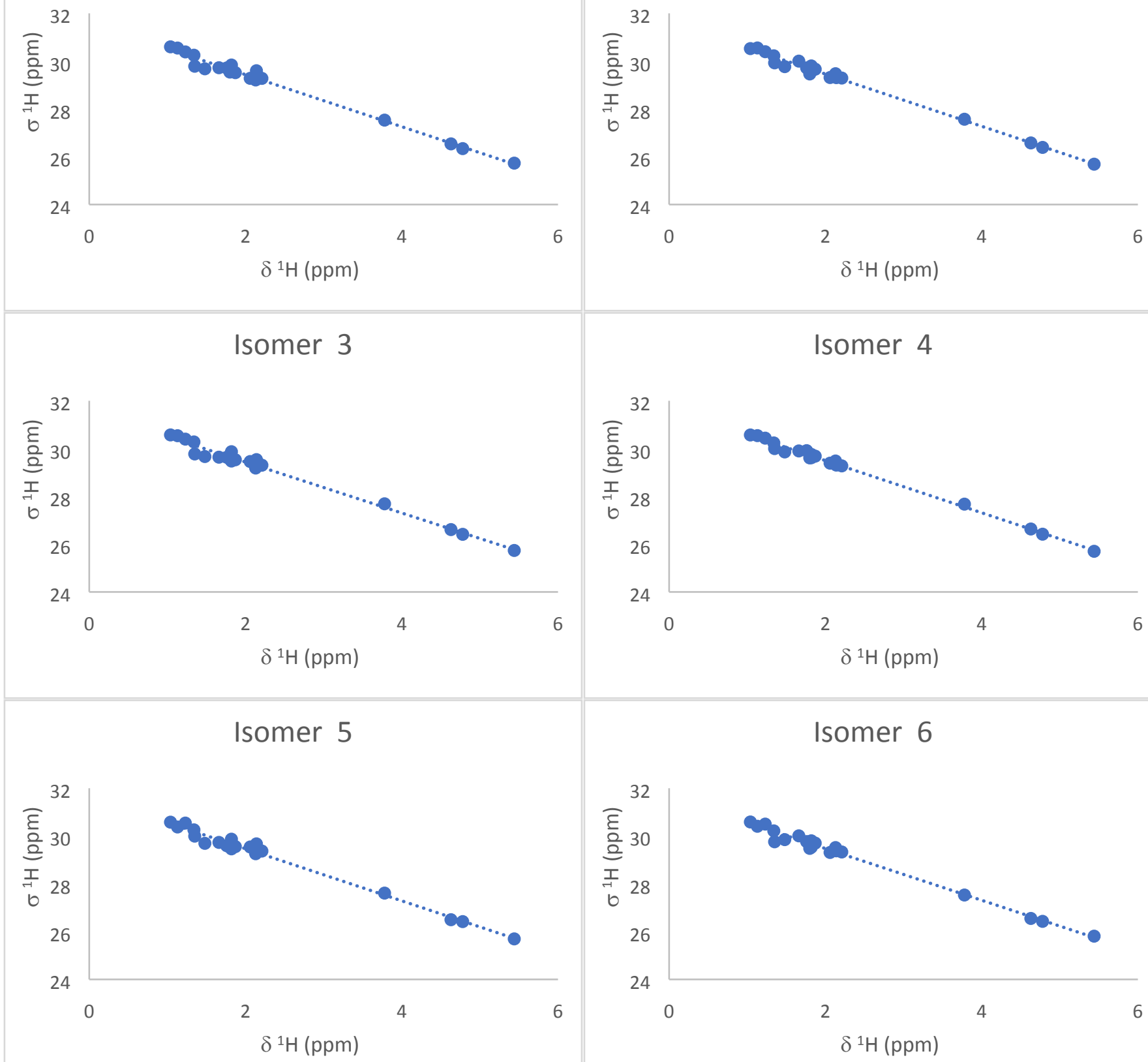

\section{Isomer 6}
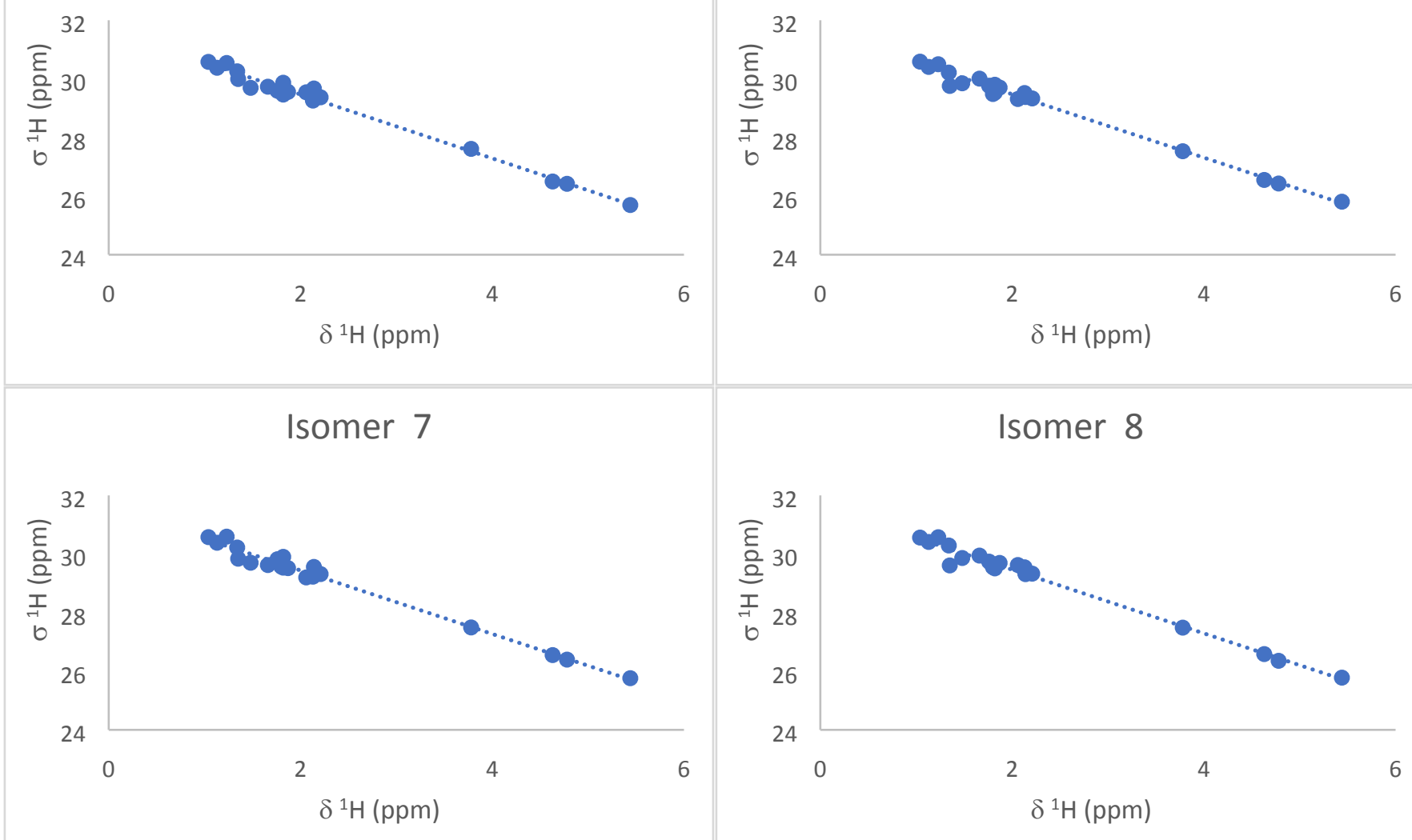
Figure S85. Compound isoelisabethatriene (vacuum) correlation plots between ${ }^{13} \mathrm{C}$ isotropic magnetic shielding values computed at the $\mathrm{PCM} / \mathrm{mPW} 1 \mathrm{PW} 91 / 6-31+\mathrm{G}^{* *}$ level of theory and experimental ${ }^{13} \mathrm{C}$ chemical shifts for isomers $1-4$

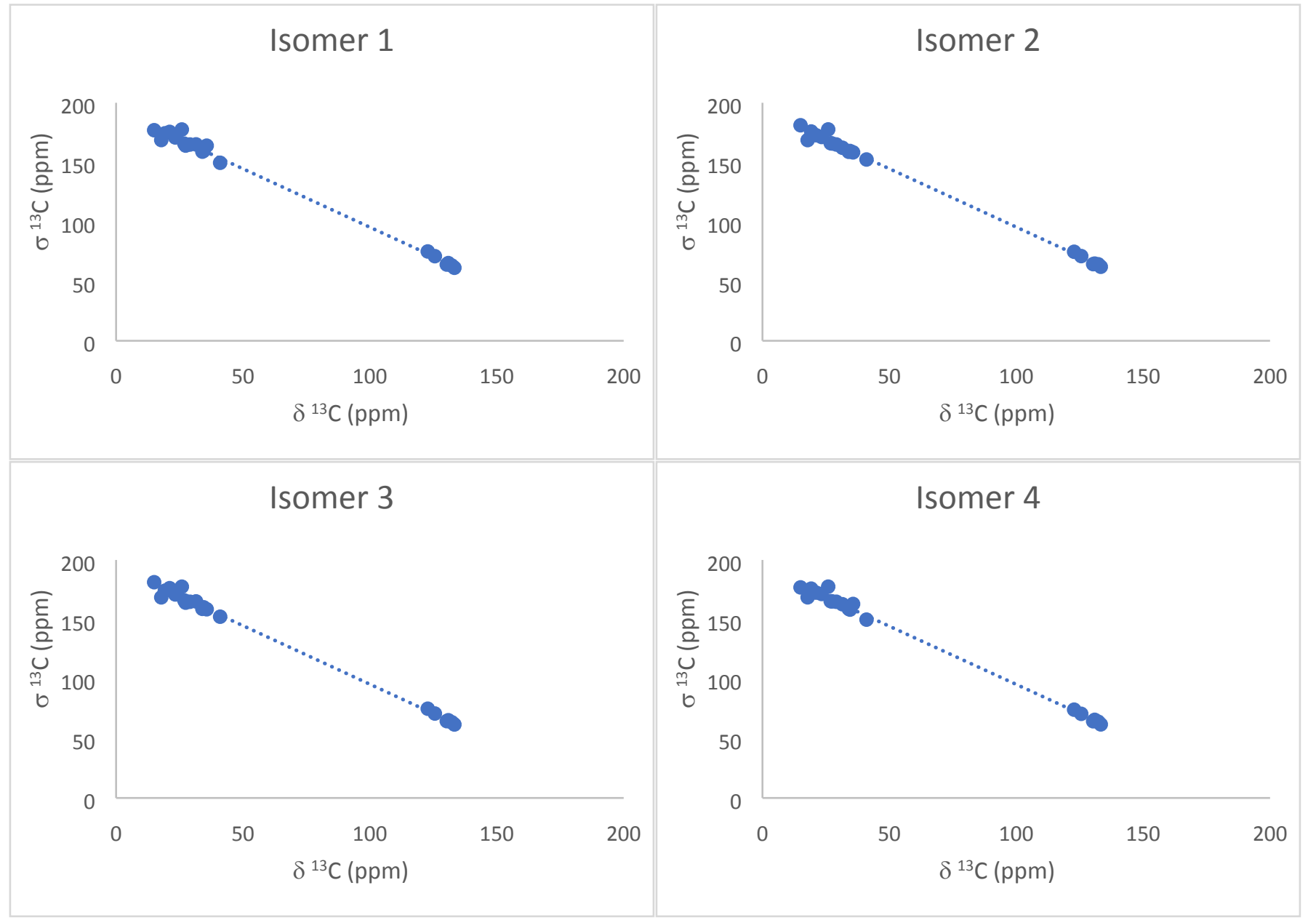


Figure S86. Compound isoelisabethatriene (vacuum) correlation plots between ${ }^{1} \mathrm{H}$ isotropic magnetic shielding values computed at the PCM/mPW1PW91/6-31+G** level of theory and experimental ${ }^{1} \mathrm{H}$ chemical shifts for isomers $1-4$

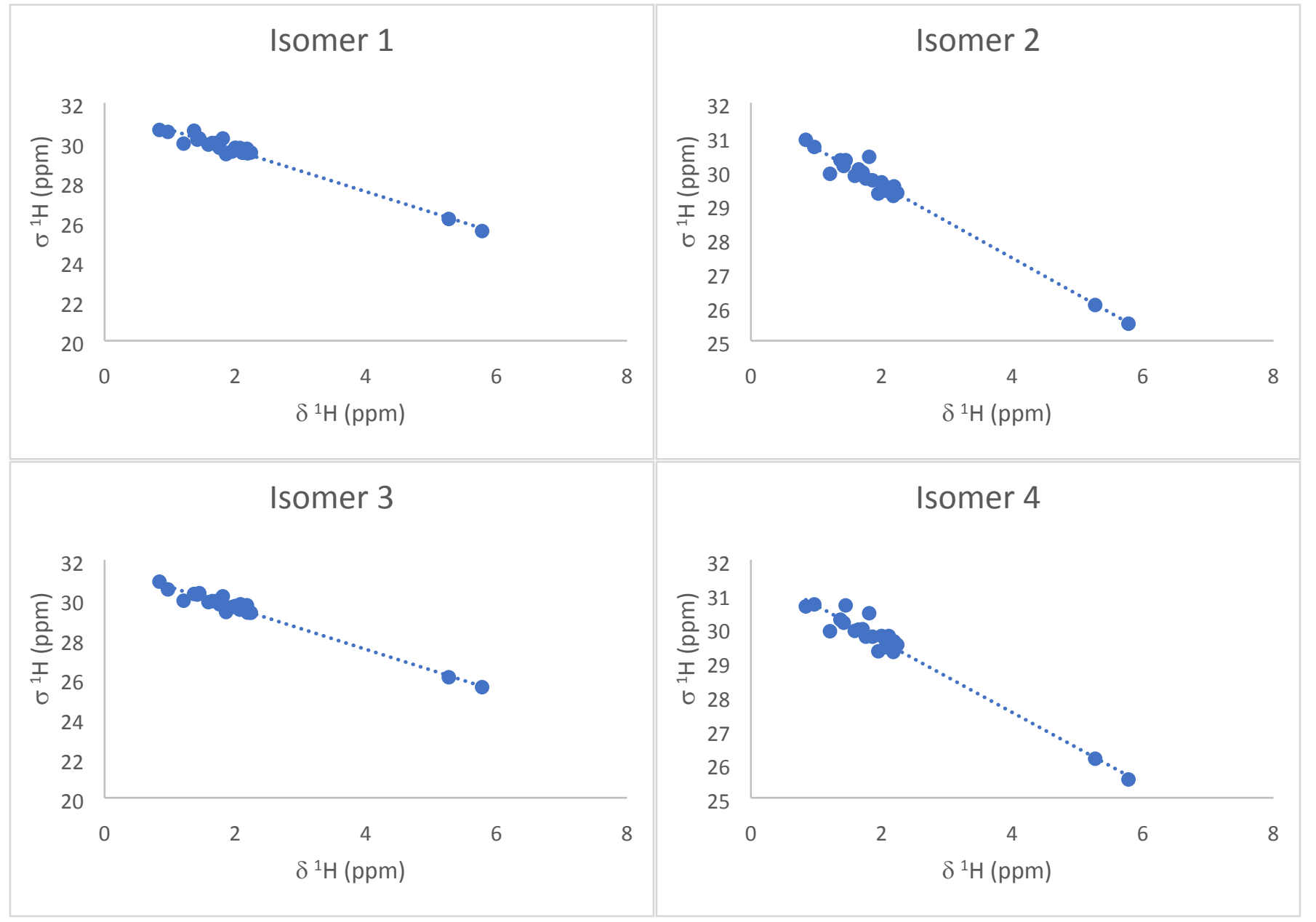


Figure S87. Compound isoelisabethatriene (benzene) correlation plots between ${ }^{13} \mathrm{C}$ isotropic magnetic shielding values computed at the $\mathrm{PCM} / \mathrm{mPW} 1 \mathrm{PW} 91 / 6-31+\mathrm{G}^{* *}$ level of theory and experimental ${ }^{13} \mathrm{C}$ chemical shifts for isomers $1-4$

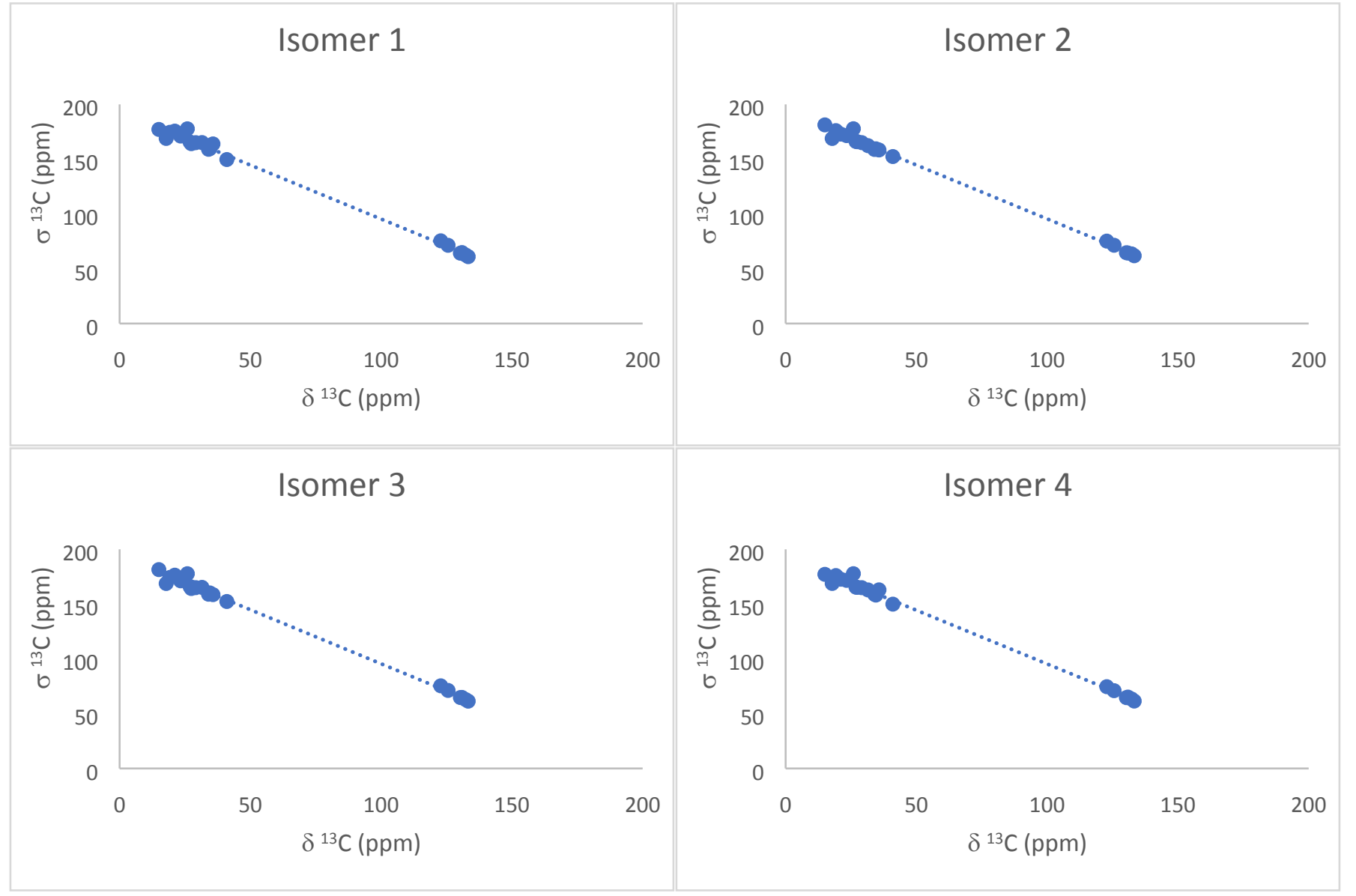


Figure S88. Compound isoelisabethatriene (benzene) correlation plots between ${ }^{1} \mathrm{H}$ isotropic magnetic shielding values computed at the PCM/mPW1PW91/6-31+G** level of theory and experimental ${ }^{1} \mathrm{H}$ chemical shifts for isomers 1-4

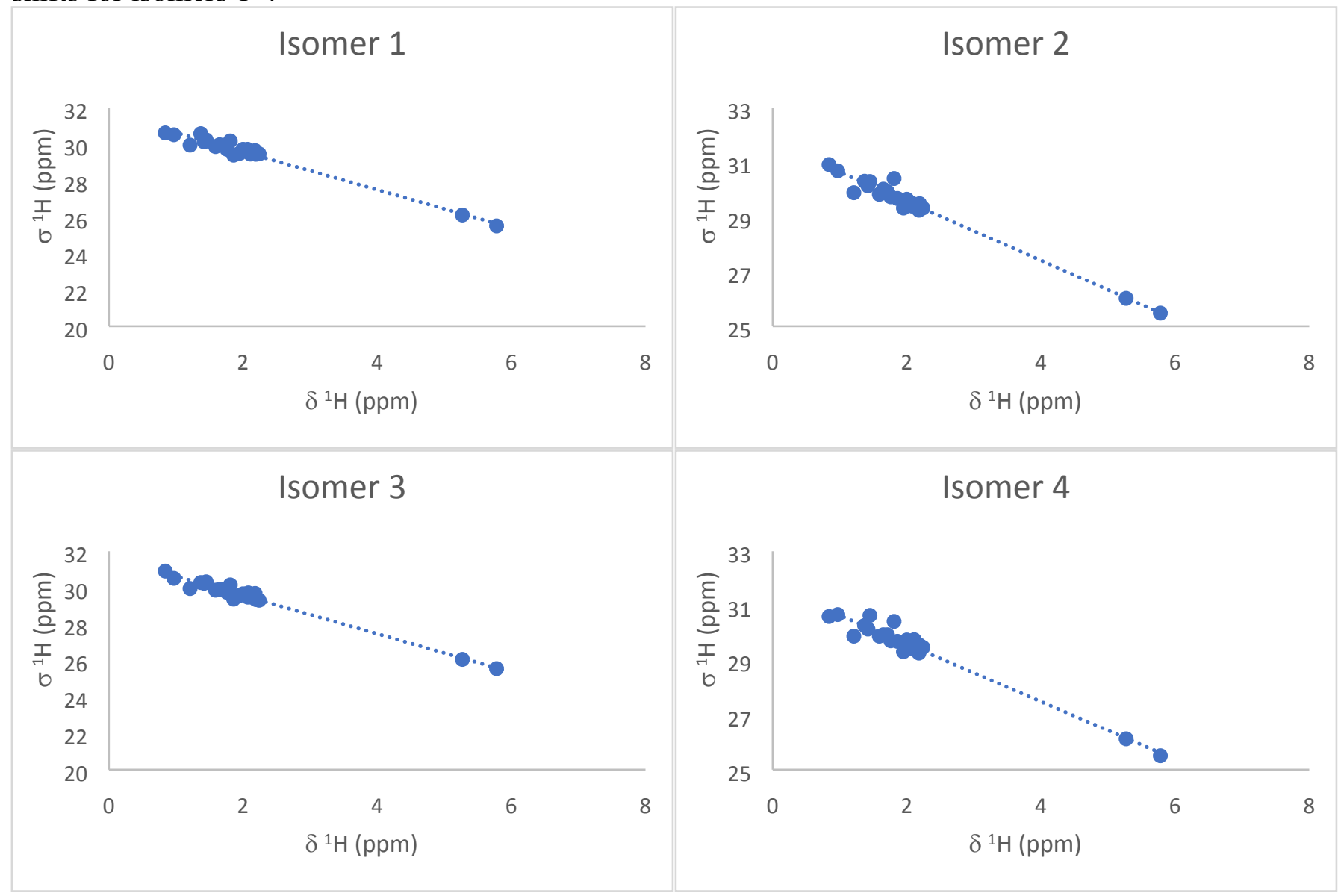

